\title{
WestVirginiaUniversity
}

THE RESEARCH REPOSITORY @ WVU

Graduate Theses, Dissertations, and Problem Reports

2015

\section{Evaluation of the antidepressant potential of dextromethorphan}

Linda Nguyen

Follow this and additional works at: https://researchrepository.wvu.edu/etd

\section{Recommended Citation}

Nguyen, Linda, "Evaluation of the antidepressant potential of dextromethorphan" (2015). Graduate

Theses, Dissertations, and Problem Reports. 6309.

https://researchrepository.wvu.edu/etd/6309

This Dissertation is protected by copyright and/or related rights. It has been brought to you by the The Research Repository @ WVU with permission from the rights-holder(s). You are free to use this Dissertation in any way that is permitted by the copyright and related rights legislation that applies to your use. For other uses you must obtain permission from the rights-holder(s) directly, unless additional rights are indicated by a Creative Commons license in the record and/ or on the work itself. This Dissertation has been accepted for inclusion in WVU Graduate Theses, Dissertations, and Problem Reports collection by an authorized administrator of The Research Repository @ WVU.

For more information, please contact researchrepository@mail.wvu.edu. 


\title{
Evaluation of the antidepressant potential of dextromethorphan
}

\author{
Linda Nguyen \\ A dissertation submitted to the School of Pharmacy at West Virginia University in partial fulfillment of \\ the requirements for the degree of \\ Doctor of Philosophy in Pharmacological and Pharmaceutical Sciences \\ Han-Ting Zhang, M.D./Ph.D., Chair \\ Rae R. Matsumoto, Ph.D. \\ David P. Siderovski, Ph.D. \\ Paola Pergami, M.D./Ph.D. \\ Patrick J. Marshalek, M.D. \\ Department of Behavioral Medicine and Psychiatry \\ Morgantown, West Virginia \\ 2015 \\ Keywords: dextromethorphan, ketamine, imipramine, dextrorphan, depression, antidepressant, sigma-1 \\ receptors, AMPA receptors, BDNF, NGF \\ (C) 2015 Linda Nguyen
}




\section{ABSTRACT \\ Evaluation of the antidepressant potential of dextromethorphan}

\section{Linda Nguyen}

Depression affects more than 350 million people and is the leading cause of disability worldwide. Major challenges in the management of depression remain the 4-12 weeks of delayed efficacy and 30\% of patients who are resistant to the therapeutic effects of current pharmaceutical treatments. We herein examined whether dextromethorphan (DM) may be able to take on these challenges, because 1) it has overlapping pharmacology with ketamine, which has shown rapid (within 24 hours) antidepressant effects, even in treatment-resistant individuals; 2) unlike ketamine with its notable abuse liability and adverse effects, DM is an over-the-counter antitussive with a high safety margin; and 3) DM binds to several protein targets which are similar to conventional medications as well as distinctive from them, affording possibilities of efficacy in treatment-refractory depression (TRD). The antidepressant-like effects of DM were determined in mice in the forced swim test (FST) and tail suspension test (TST), the two most validated animal models for predicting antidepressant actions. Next, the role of sigma-1 and AMPA receptors in the antidepressant-like effects of DM was examined because mounting evidence suggests that these novel mechanisms contribute to a faster onset of antidepressant efficacy. We also assessed in vivo and in vitro whether DM can promote neural adaptations, which is thought to be a final common pathway for fast acting and conventional antidepressants. Finally, since DM undergoes substantial first-pass metabolism to active metabolites, we evaluated the impact of metabolism on the antidepressant-like effects of DM to determine whether the DM effects are likely mediated through itself or its major metabolites. Our results revealed that DM produces antidepressant-like actions in both the FST and TST, similar to the fast acting and conventional antidepressant controls represented by ketamine and imipramine. Whereas the antidepressant-like effects of DM were blocked by pretreatment with a sigma-1 receptor antagonist (BD1063) or AMPA receptor antagonist (NBQX), the antidepressant-like effects of ketamine were blocked with only NBQX; neither antagonists attenuated the antidepressant-like effects of imipramine. This indicates while only AMPA receptors may play a significant role in mediating the antidepressant-like behaviors of ketamine, both AMPA and sigma-1 receptors may have pivotal roles for DM. Moreover, ketamine rapidly increased hippocampal pro-BDNF levels (within 40 minutes), whereas DM and imipramine did not alter pro-BDNF or BDNF levels in mouse hippocampus or frontal cortex, two key brain regions implicated in depression, in the same time frame. In an in vitro model of neurite growth, however, all three drugs potentiated NGF-induced neurite outgrowth, suggesting DM may work through other trophic factors (e.g., NGF) to facilitate antidepressant-relevant neural adaptations. Lastly, slowing down the metabolism of DM potentiated its antidepressant-like effects, indicating DM rather than its metabolites is the primary mediator of the antidepressant-like effects observed herein. Overall, our results indicate DM produces antidepressant-like effects through mechanisms that overlap in part with ketamine. Taken together with a recent clinical report of DM's efficacy (some within 1-2 days) for treatment-resistant bipolar depression, these data suggest DM may not only elicit antidepressant effects faster than the currently approved medications, but also have efficacy in TRD. 


\section{ACKNOWLEDGEMENTS}

This dissertation could not have been completed without the great support that I have received from many people over the "long" and arduous two years.

First and foremost, I would like to express my deep and sincere gratitude to my advisor, Dr. Rae

R. Matsumoto. Without her encouragement and mentorship, none of what is included in the current dissertation would have been possible, nor would I have been able to complete the work in such a short amount of time. Dr. Matsumoto has been a constant source of inspiration and motivation to pursue many of the opportunities presented to me as a graduate student. Even before I officially started my graduate training in her lab, she had encouraged me to write my very first, first-author original research paper. In addition, her guidance and advice on matters that extend beyond the benchtop has allowed me to grow not only as a scientist, but also as an individual.

Secondly, I wish to thank the remainder of my committee members, Dr. Han-Ting Zhang, Dr. Paola Pergami, Dr. David P. Siderovski, and Dr. Patrick J. Marshalek for their support, guidance, and helpful suggestions. I would especially like to thank Dr. Siderovski, for being my go-to-person currently at WVU when in need; Dr. Zhang, for giving me a new lab home; and Dr. Marshalek for partaking in the journey with me to translate my preclinical findings into a clinical study (or at least try to) at WVU.

I would also like to thank past members of the Matsumoto lab: Jason Healy, Ying Zhang, and especially Matthew Robson, for training me in my very first mouse depression study, and Anna Scandinaro, for holding down the Matsumoto lab fort with me while it lasted. Special thanks to Brandon Lucke-Wold, for his collaborative spirit as we trekked through the graduate training of the M.D./Ph.D. program together; and Dr. Jeffrey Wimsatt, for believing in my abilities to get to the "heart" of the matter (or at least the mouse) and allowing me take part in several mouse pharmacokinetic studies.

Lastly, I would like to say thank you to my family for all the years of unconditional love and never-ceasing support, and my friends for helping me maintain some social balance in my life. 


\section{DEDICATION}

This dissertation is dedicated to my loving parents, whose own perseverance and hard work inspires me to pursue my own dreams with great gratitude and passion. 


\section{TABLE OF CONTENTS}

\section{ABSTRACT}

ACKNOWLEDGEMENTS

DEDICATION

TABLE OF CONTENTS

LIST OF FIGURES

LIST OF TABLES

LIST OF ABBREVIATIONS

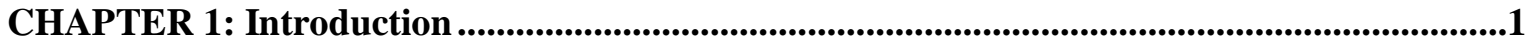

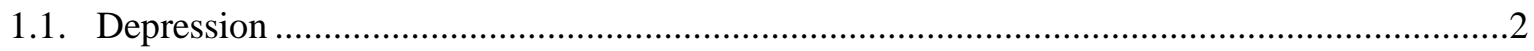

1.2. Available treatments for depression and their limitations .................................................. 3

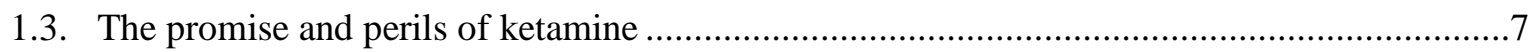

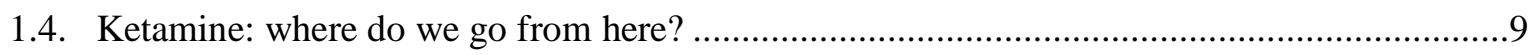

1.5. Dextromethorphan (DM) as a logical next step ........................................................... 10

CHAPTER 2: DM as a potential safe and effective antidepressant .............................................12

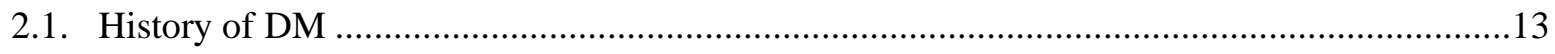

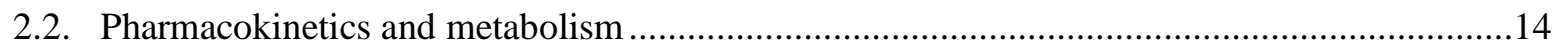

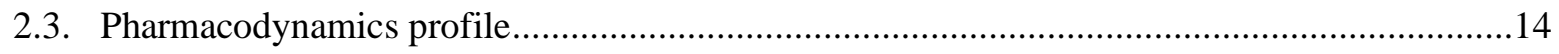

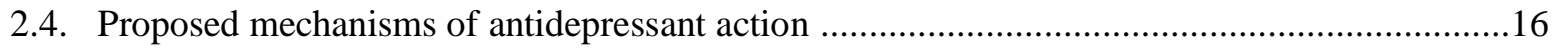

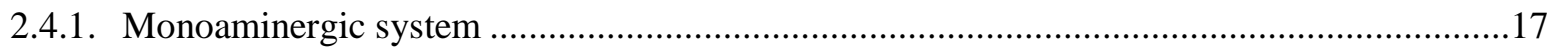

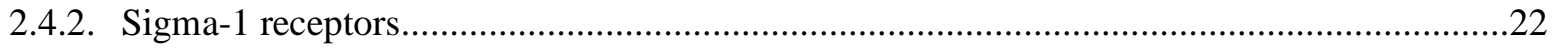

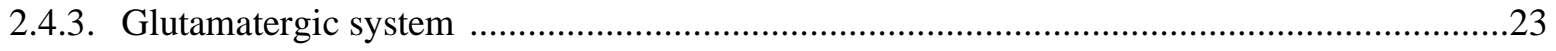

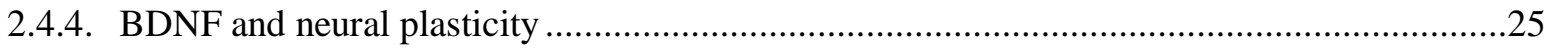

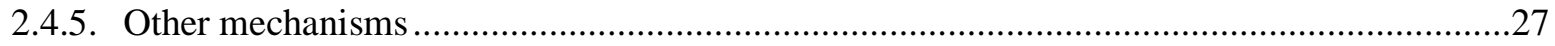

2.5. Potential impact of metabolism on antidepressant action ....................................................29

2.6. Concerns about abuse liability and long-term toxicity ….................................................... 30 
2.7. Clinical evidence of antidepressant efficacy .

CHAPTER 3: Involvement of sigma-1 receptors in the antidepressant-like effects of DM .......35

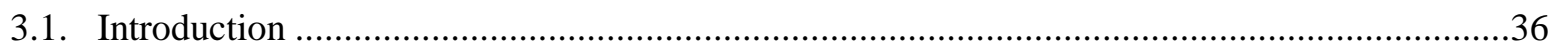

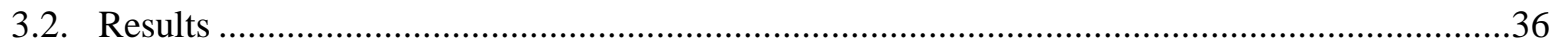

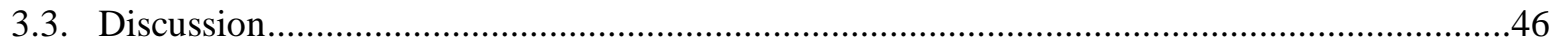

CHAPTER 4: Involvement of AMPA receptors in the antidepressant-like effects of DM........52

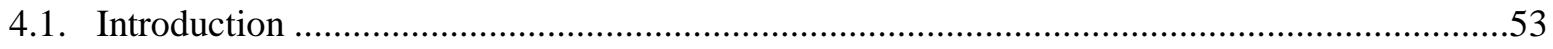

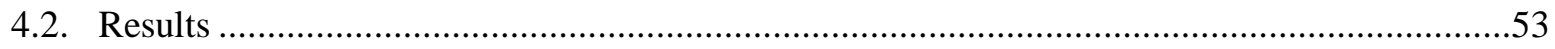

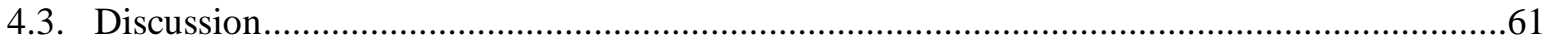

CHAPTER 5: Behavioral and biochemical analyses of effects of DM in relation to its

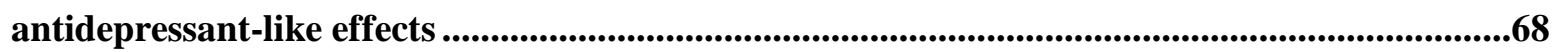

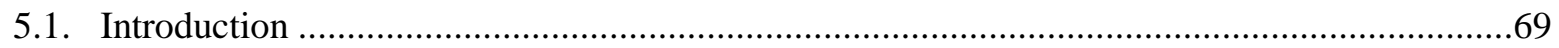

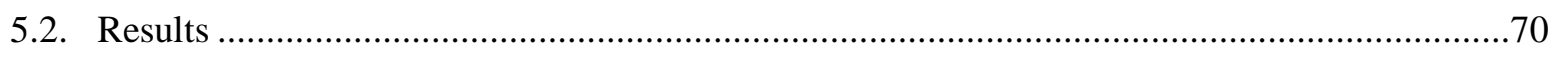

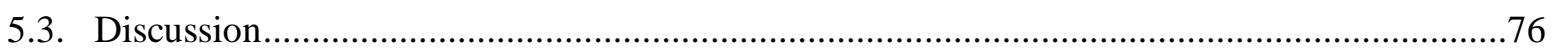

CHAPTER 6: Potentiation of nerve growth factor-induced neurite outgrowth by DM............81

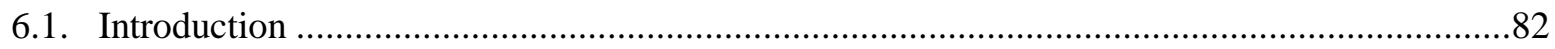

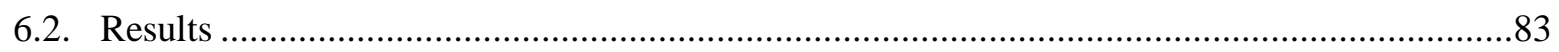

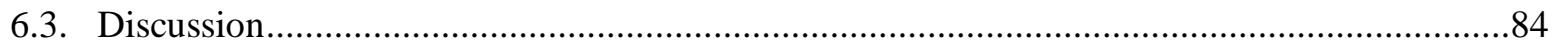

CHAPTER 7: Evaluation of the antidepressant-like effects of deuterated DM.........................91

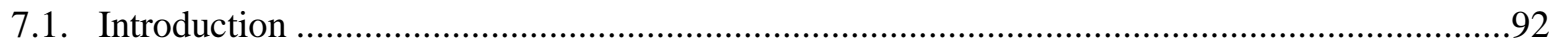

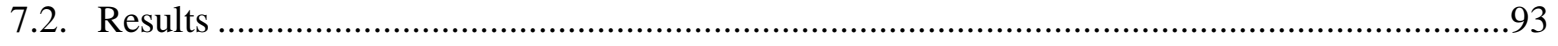

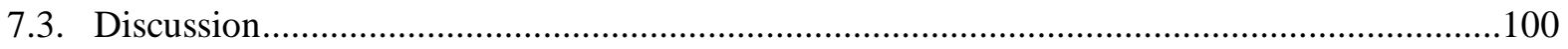

CHAPTER 8: Evaluation of the antidepressant-like effects of dextrorphan ................................104

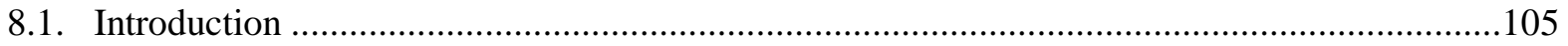

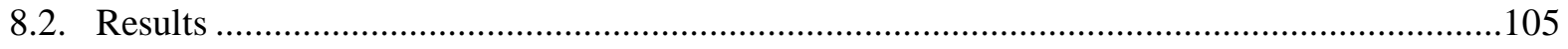

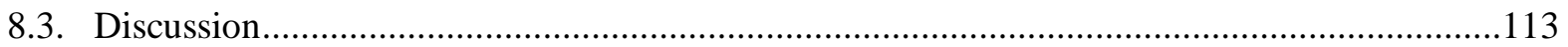




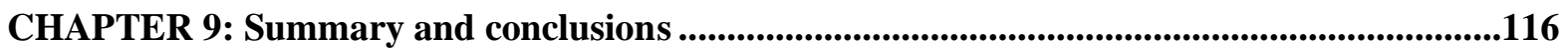

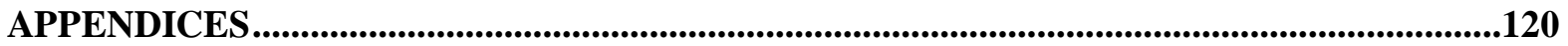

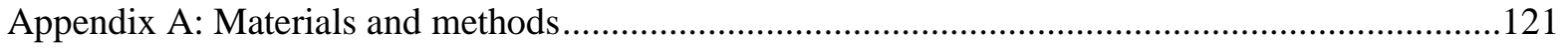

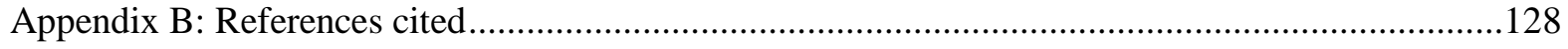

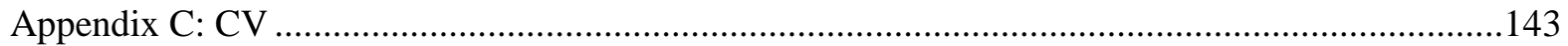




\section{LIST OF FIGURES}

Figure 1. DM demethylation pathways catalyzed by CYP2D6 and CYP3A4 …..........................15

Figure 2. Comparison of pharmacologic actions of DM, ketamine, and imipramine ....................20

Figure 3. DM may promote antidepressant-relevant neural plasticity through sigma-1 and AMPA

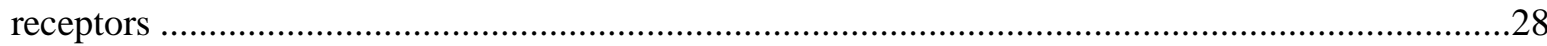

Figure 4. Antidepressant-like effects of imipramine, ketamine, and DM in the FST ....................37

Figure 5. Blockade of sigma-1 receptors prevented the antidepressant-like effects of DM, but not

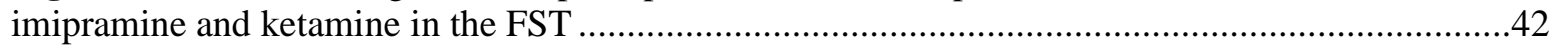

Figure 6. Competitive antagonism of the behavioral effects of DM by BD1063 in the FST ...........44

Figure 7. Potentiation of the antidepressant-like effects of DM by quinidine in the FST ................47

Figure 8. Antidepressant-like effects of imipramine, ketamine, and DM in the TST ....................54

Figure 9. Potentiation of the antidepressant-like effects of DM by quinidine in the TST...............56

Figure 10. Blockade of AMPA receptors prevented the antidepressant-like effects of ketamine, DM, and

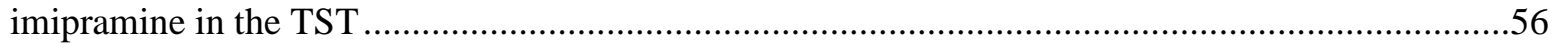

Figure 11. Blockade of AMPA receptors prevented the antidepressant-like effects of ketamine and DM,

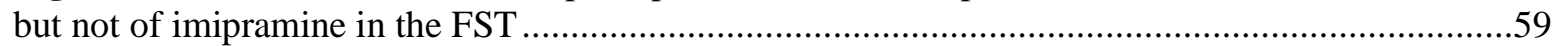

Figure 12. Antidepressant-like effects of ketamine, imipramine, and DM in the FST using previously

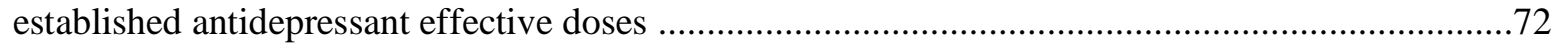

Figure 13. Effects of ketamine, imipramine, and DM in the OFT ...........................................72

Figure 14. Effects of ketamine, imipramine, and DM on pro-BDNF levels in mouse hippocampus or frontal cortex

Figure 15. Effects of ketamine, imipramine, and DM on BDNF levels in mouse hippocampus or frontal cortex

Figure 16. Blockade of AMPA receptors prevented the ketamine-induced behaviors in the FST and OFT, and increase in hippocampal pro-BDNF levels .75

Figure 17. Proposed signaling pathways involved in stimulation of neurite outgrowth by ketamine and DM . .85

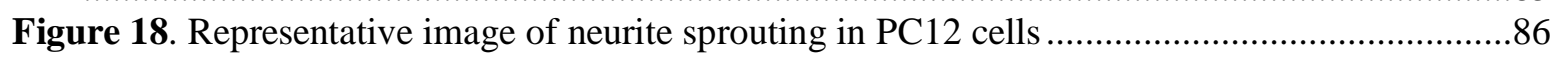

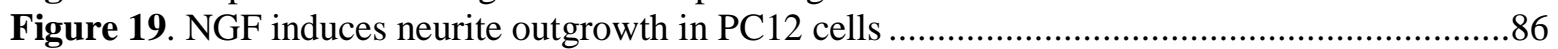

Figure 20. Imipramine potentiates NGF-induced neurite outgrowth in PC12 cells .......................87

Figure 21. Ketamine potentiates NGF-induced neurite outgrowth in PC12 cells ...........................87

Figure 22. DM does not induce neurite outgrowth alone but potentiates NGF-induced neurite outgrowth in PC12 cells .88

Figure 23. Antidepressant-like and stimulatory effects of d6-DM in the FST, TST and OFT.........94 Figure 24. Blockade of AMPA or sigma-1 receptors attenuated the stimulatory effects, but not antidepressant-like effects of d6-DM. .96 Figure 25. Co-administration of quinidine attenuated the stimulatory effects, but had no significant effects on the antidepressant-like effects of d6-DM.

Figure 26. Antidepressant-like and stimulatory effects of DX in the FST, TST and OFT...............106 Figure 27. Blockade of sigma-1 receptors failed to attenuate the antidepressant-like effects of DX in the FST and stimulatory effects in the OFT. 109

Figure 28. Blockade of AMPA receptors failed to attenuate the antidepressant-like effects of DX in the FST and TST, but blocked the stimulatory effects in the OFT

Figure 29. Illustrations of FST, TST, and OFT 


\section{LIST OF TABLES}

Table 1. Currently approved treatments for depression .....................................................

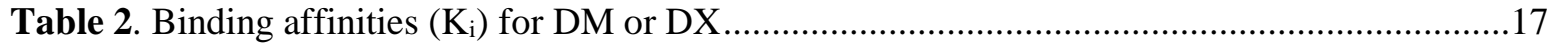

Table 3. Binding parameters for sigma-1 receptors in the absence and presence of DM.................47

Table 4. Effects of various treatments on locomotor activity in the OFT ....................................62 


\section{LIST OF ABBREVIATIONS}

\begin{tabular}{|c|c|}
\hline 3-HM & 3-hydroxymorphinan \\
\hline 3-MM & 3-methoxymorphinan \\
\hline $5-\mathrm{HT}$ & Serotonin \\
\hline AMPA & Alpha-amino-3-hydroxy-5-methyl-4-isoxazolepropionic acid \\
\hline AUC & Area Under the Curve \\
\hline BCA & Bicinchoninic Acid \\
\hline BD1047 & N'-[2-(3,4-Dichlorophenyl)ethyl]-N-methyl-2-(dimethylamino)ethylamine \\
\hline BD1063 & 1-[2-(3,4-Dichlorophenyl)ethyl]-4-methylpiperazine \\
\hline BDNF & Brain-Derived Neurotrophic Factor \\
\hline bFGF & Basic Fibroblastic Growth Factor \\
\hline BPRS & Brief Psychiatric Rating Scale \\
\hline CADSS & Clinician-Administered Dissociative States Scale \\
\hline cAMP & Cyclic Adenosine Monophosphate \\
\hline CGI-I & Clinical Global Impression-Improvement \\
\hline Cmax & Maximum (Peak) Serum Concentration \\
\hline CNS & Central Nervous System \\
\hline CYP & Cytochrome P450 \\
\hline d & $\operatorname{Day}(s)$ \\
\hline d6 & Deuterium \\
\hline DM & Dextromethorphan \\
\hline DPBS & Dulbecco's Phosphate-Buffered Saline \\
\hline DSM-V & Diagnostic and Statistical Manual of Mental Health, Fifth Edition \\
\hline $\mathrm{DX}$ & Dextrorphan \\
\hline ECT & Electroconvulsive Treatment \\
\hline EM & Extensive Metabolizer \\
\hline EMA & European Medicines Agency \\
\hline FDA & Food and Drug Administration \\
\hline FST & Forced Swim Test \\
\hline $\mathrm{h}$ & Hour(s) \\
\hline HDRS & Hamilton Depression Rating Scale \\
\hline $\mathrm{IM}$ & Intermediate Metabolizer \\
\hline i.p. & Intraperitoneal \\
\hline i.v. & Intravenous \\
\hline LTD & Long-Term Depression \\
\hline LTP & Long-Term Potentiation \\
\hline MAM & Mitochondrial-Associated Endoplasmic Reticulum Membrane \\
\hline MAOI & Monoamine Oxidase Inhibitor \\
\hline MAPK & Mitogen-Activated Protein Kinase \\
\hline MDD & Major Depressive Disorder \\
\hline MEK & Mitogen-Activated Protein Kinase \\
\hline MES & 2-(N-morpholino)ethane Sulfonic Acid \\
\hline $\min$ & Minute(s) \\
\hline NBQX & 2,3-Dihydroxy-6-Nitro-7-Sulfamoylbenzo[f]quinoxaline \\
\hline $\mathrm{NE}$ & Norepinephrine \\
\hline NET & Norepinephrine Transporter \\
\hline NGF & Nerve Growth Factor \\
\hline NMDA & N-methyl-D-asparate \\
\hline OFT & Open Field Test \\
\hline OTC & Over-the-Counter \\
\hline
\end{tabular}




$\begin{array}{ll}\text { p75 } & \text { p75 } \\ \text { PBA } & \text { Pseudobulbar Affect } \\ \text { PC12 } & \text { Pheochromocytoma } \\ \text { PCP } & \text { Phencyclidine } \\ \text { PFC } & \text { Prefrontal Cortex } \\ \text { PM } & \text { Poor Metabolizer } \\ \text { PRISE } & \text { Patient-Rated Inventory of Side Effects } \\ \text { PTSD } & \text { Post-Traumatic Stress Disorder } \\ \text { PVDF } & \text { Polyvinylidene Difluoride } \\ \text { RCT } & \text { Randomized Controlled Trial } \\ \text { RPMI } & \text { Roswell Park Memorial Institute } \\ \text { SA4503 } & \text { 1-[2-(3,4-Dimethoxyphenyl)ethyl]-4-(3-phenylpropyl)piperazine } \\ \text { SAFTEE-SI } & \text { Systematic Assessment For Treatment Emergent Effects Self-Report Inventory } \\ \text { SDS } & \text { Sodium Dodecyl Sulfate } \\ \text { SERT } & \text { Serotonin Transporter } \\ \text { SNRI } & \text { Serotonin-Norepinephrine Reuptake Inhibitor } \\ \text { SSRI } & \text { Selective Serotonin Reuptake Inhibitors } \\ \text { TCA } & \text { Tricyclic Antidepressant } \\ \text { TRD } & \text { Treatment-Refractory Depression } \\ \text { TrkA } & \text { Tropomyosin Receptor Kinase A } \\ \text { TrkB } & \text { Tropomyosin Receptor Kinase B } \\ \text { TST } & \text { Tail Suspension Test } \\ \text { VGCC } & \text { Voltage Gated Calcium Channel } \\ \text { VPA } & \text { Valproic Acid } \\ \text { YMRS } & \text { Young Mania Rating Scale } \\ & \end{array}$




\section{CHAPTER 1}

\section{Introduction}




\subsection{Depression}

Affecting up to 350 million people and standing as the leading cause of disability worldwide (WHO, 2012), depression is a serious, debilitating and costly mental illness. Also called major depression, major depressive disorder (MDD) or clinical depression, in the US, it affects 5-12\% of men and 10-25\% of women during their lifetime (Kessler et al., 2003; Valenstein et al., 2001); and many more have depressive disorders that fall short of the criteria for MDD. According to the Diagnostic and Statistical Manual of Mental Health, Fifth Edition (DSM-V), the diagnosis for MDD requires either depressed mood or anhedonia, along with five or more of the following symptoms: 1) sad or irritable mood; 2) loss of interest in most activities; 3) fluctuations in weight and/or appetite; 4) insomnia or hypersomnia, 5) psychomotor agitation or retardation; 6) fatigue or loss of energy; 7) inappropriate guilt or feelings of worthlessness; 8) diminished attention and/or cognition; and 9) recurrent thoughts of death and/or suicide ideations (American Psychiatric et al., 2013). These symptoms must occur nearly every day throughout a 2-week period which cannot be explained by other medical conditions (American Psychiatric et al., 2013). A small percentage of patients with MDD have had or will have manic episodes consisting of hyperactivity, euphoria, and an increase in pleasure seeking (Belmaker, 2004). Although the underlying pathophysiology in these cases and in cases of MDD have some overlap, a history of mania defines a distinct illness termed bipolar disorder (Belmaker, 2004).

MDD is defined by the occurrence of at least one major depressive episode; but for most people, MDD is a life-long episodic disorder with multiple recurrences (average one episode in every 5-year period), with approximately 20-25\% of MDD patients experiencing a chronic, unremitting course (Mueller and Leon, 1996). The chronic and recurrent course of MDD is a major clinical issue, often requiring long-term prophylactic treatment (Fava and Kendler, 2000). This adds to the substantial economic burden imposed by MDD, which is associated with functional impairment and decreased productivity. In the US direct costs of depression were \$26 billion while indirect costs accounted for more than $\$ 50$ billion in 2000 (Wade and Haring, 2010). 


\subsection{Available treatments for depression and their limitations}

Several approved treatment approaches to MDD are currently available. These approaches include antidepressant medications, psychotherapy, electroconvulsive treatment (ECT), and other somatic therapies. Table 1 summarizes the advantages and disadvantages of these treatments.

In the realm of psychotherapy, two types of time-limited (typically up to 16 weeks) psychotherapy have been shown consistently to be effective in treating MDD: interpersonal psychotherapy and cognitive behavioral therapy. While psychotherapy and pharmacological interventions in primary care have comparable efficacy in reducing symptoms of mild to moderate depression (Wolf and Hopko, 2008), combined therapy with pharmacotherapy is considered to be superior to psychotherapy alone for treatment of more severe, recurrent depressions (Thase et al., 1997).

Many randomized controlled trials (RCTs) have established the efficacy of antidepressants and ECT for MDD. Unfortunately, in addition to the need to administer the drugs for weeks or months before seeing clinical benefits, up to two-thirds of patients remain symptomatic following first-line treatment (Trivedi et al., 2006), and up to a third of patients show little to no improvement despite multiple treatments (Schlaepfer et al., 2012). This latter group is said to have treatment-refractory depression (TRD), often defined as a failure to produce significant clinical improvement from at least two trials with antidepressants from different pharmacological classes (adequate in dose, duration, and compliance) (Berlim and Turecki, 2007). Since the mid-1960s, a large body of evidence has concluded that ECT is the most effective acute treatment for MDD. In studies that have ECT with placebo and antidepressant medications, approximately $70-80 \%$ of patients respond to ECT, $30-52 \%$ to antidepressants, and $25-45 \%$ to placebo (Association, 2008). Moreover, about 50\% of patients show therapeutic response by session 3 (within 1 week) of ECT (Husain et al., 2004; Shapira and Lerer, 1999). Despite the superior and quicker onset of efficacy with ECT, cognitive and memory side effects can be significant and sometimes persistent (Association, 2008; Sackeim et al., 2007). The effectiveness of other and more recently approved somatic therapies (vagus nerve stimulation and repetitive transcranial magnetic stimulation in 2005 and 2008, respectively) has not been as well-established (Holtzheimer and Mayberg, 2011). 
Table 1. Currently approved treatments for depression. 5-HT, serotonin; NE, norepinephrine; DA, dopamine; CNS, central nervous system.

\begin{tabular}{|c|c|c|c|}
\hline Treatment & $\begin{array}{l}\text { Proposed mechanism of action } \\
\text { (or procedural description) }\end{array}$ & Advantage & Disadvantage \\
\hline \multicolumn{4}{|l|}{ Pharmacotherapy } \\
\hline $\begin{array}{l}\text { 5-HT reuptake inhibitors } \\
\text { (SSRIs): } \\
\text { Citalopram, escitalopram, } \\
\text { fluoxetine, fluvoxamine, } \\
\text { paroxetine, sertraline }\end{array}$ & $\begin{array}{l}\text { Selectively inhibits reuptake of 5- } \\
\text { HT }\end{array}$ & $\begin{array}{ll}\text { - } & \text { First-line treatment choice } \\
\text { - } & \text { Low side-effect profile } \\
& \text { (especially vs. tricyclics) }\end{array}$ & $\begin{array}{ll}- & \text { Sexual side effects (delayed } \\
\text { ejaculation, anorganismia) } \\
\text { - } & \text { Weight gain } \\
\text { - } & \text { CNS effects (insomnia, } \\
\text { somnolence, temporary } \\
\text { anxiety, headache) } \\
\text { - 5-HT syndrome }\end{array}$ \\
\hline $\begin{array}{l}\text { 5-HT/NE reuptake inhibitors } \\
\text { (SNRIs): } \\
\text { Desvenlafaxine, duloxetine, } \\
\text { venlafaxine, levomilnacipran }\end{array}$ & Inhibits reuptake of 5-HT and NE & $\begin{array}{l}\text { Low side-effect profile } \\
\text { (especially vs. tricyclics) }\end{array}$ & $\begin{array}{l}\text { - Sexual dysfunction, weight } \\
\text { gain, insomnia, somnolence } \\
\text { - } \begin{array}{l}\text { Dose-related increase in blood } \\
\text { pressure }\end{array}\end{array}$ \\
\hline $\begin{array}{l}\text { Monoamine oxidase (MAO) } \\
\text { inhibitors: } \\
\text { Isocarboxazid, phenylzine, } \\
\text { tranylcypromine, selegiline } \\
\text { (selective for MAO-B) }\end{array}$ & $\begin{array}{l}\text { Nonselectively inhibits enzymes } \\
\text { (MAO-A and MAO-B) involved in } \\
\text { breakdown of monoamines }\end{array}$ & $\begin{array}{l}\text { Particularly effective in } \\
\text { atypical MDD (i.e., mood } \\
\text { reactivity accompanied by } \\
\text { hyperphagia, hypersomnia, } \\
\text { extreme fatigue, and/or } \\
\text { hypersensitivity to rejection) } \\
\text { - Some efficacy in treatment- } \\
\text { refractory cases }\end{array}$ & $\begin{array}{l}\text { Dietary restriction: avoid } \\
\text { tyramine containing foods } \\
\text { (e.g., aged cheese, red wine, } \\
\text { and certain meats) } \\
\text { Irreversible enzyme inhibition } \\
\text { requires careful considerations } \\
\text { when taking other drugs }\end{array}$ \\
\hline $\begin{array}{l}\text { Tricyclic and tetracyclic } \\
\text { antidepressants: } \\
\text { Amitriptyline, desipramine, } \\
\text { doxepin, imipramine, maprotiline, } \\
\text { nortriptyline, protriptyline, } \\
\text { trimipramine }\end{array}$ & $\begin{array}{l}\text { Nonselectively inhibits reuptake of } \\
\text { monoamines }\end{array}$ & $\begin{array}{l}\text { Used to be gold standard of } \\
\text { pharmacological treatment }\end{array}$ & $\begin{array}{l}\text { Considerable off-target } \\
\text { antagonist effects (histamine } \\
\text { H1, alpha adrenergic, and } \\
\text { muscarinic receptors) } \\
\text { - } \quad \begin{array}{l}\text { Respiratory depression and } \\
\text { arrhythmia can be fatal }\end{array} \\
\end{array}$ \\
\hline $\begin{array}{l}\text { NE/DA reuptake inhibitor: } \\
\text { Bupropion }\end{array}$ & $\begin{array}{l}\text { Mild DA \& NE reuptake inhibitor } \\
(\mathrm{DA}>\mathrm{NE}) \text {; nicotinic acetylcholine } \\
\text { receptor antagonist }\end{array}$ & $\begin{array}{ll}\text { - } & \text { Little or no sexual side effects } \\
\text { or weight gain } \\
\text { - } & \text { Used for smoking cessation } \\
\text { - } & \text { Used in combination with } \\
& \text { SSRI to treat SSRI-induced } \\
\text { sexual dysfunction }\end{array}$ & $\begin{array}{l}\text { - } \text { Agitation, anxiety, restlessness, } \\
\text { insomnia, constipation, dry } \\
\text { mouth } \\
\text { Increased seizure risk } \\
\text { (contraindicated in bulimic } \\
\text { patients and patients with } \\
\text { epilepsy) } \\
\text { Twice daily dosing }\end{array}$ \\
\hline
\end{tabular}


5-HT antagonist and reuptake inhibitor:

Trazadone, nefazodone

Noradrenergic and specific serotonergic modulator:

Mirtazapine 5-HT1A receptor partial agonist:

Vilazodone
Antagonism of 5-HT2A, 5-HT2C and alpha- 1 adrenergic receptors; nefazodone also inhibits NE reuptake

Inhibits the alpha- 2 adrenergic autoreceptor causing increase release of 5-HT and NE; antagonizes post-synaptic 5-HT2 \& 5-HT3 receptors, disinhibiting release of DA \& NE in reward circuitry
Potently and selectively inhibits 5-

HT reuptake; acts as a partial agonist at the 5-HT1A receptor
- Trazadone used for insomnia at low dose with another antidepressant agent on-board

Trazadone: sedation, dizziness, priapism

- Nefazodone: black box warning of hepatotoxicity; strong CYP3A4 inhibitor; twice daily dosing

- May have faster onset of antidepressant action (as early as first week of treatment)

- Antagonist at histamine H1 receptors, causing sedation and weight gain

\section{Psychotherapy

Cognitive behavioral therapy

Interpersonal psychotherapy

Restructures negative thought

patterns

Focuses on the relationships

between a person and significant others
- $\quad$ Few sexual side effects

$+\frac{10}{2}$

- $\quad$ First-line treatment for mild to moderate depression in youths

- Skills acquired can be extended to coping with future stresses and difficulties even after the treatment has finished
- $\quad$ Not effective in very severe depression

- Time consuming, especially CBT

- High motivation and commitment required on part of both patient and therapist

\begin{tabular}{|c|c|c|c|}
\hline \multicolumn{4}{|l|}{ Somatic therapy } \\
\hline Electroconvulsive therapy & $\begin{array}{l}\text { Application of an electric stimulus } \\
\text { to the surface of the head, with the } \\
\text { aim of inducing a seizure }\end{array}$ & $\begin{array}{l}\text { Most effective treatment for } \\
\text { MDD to date } \\
\text { - Indicated specifically in severe } \\
\text { psychotic depression }\end{array}$ & $\begin{array}{l}\text { - } \quad \text { Cardiac arrhythmias and blood } \\
\text { pressure changes } \\
\text { - } \\
\text { - Acute cognitive impairments } \\
\text { Anterograde or retrograde } \\
\text { amnesia }\end{array}$ \\
\hline $\begin{array}{l}\text { Repetitive transcranial magnetic } \\
\text { stimulation }\end{array}$ & $\begin{array}{l}\text { Short electromagnetic pulses are } \\
\text { administered through an } \\
\text { electromagnetic coil held against } \\
\text { the forehead, causing small } \\
\text { electrical currents that stimulate } \\
\text { nerve cells in a specific brain area }\end{array}$ & $\begin{array}{ll}\text { - } & \text { Noninvasive } \\
\text { - } & \text { Can safely be done as an } \\
& \text { outpatient procedure }\end{array}$ & - $\quad$ Headaches or scalp discomfort \\
\hline
\end{tabular}


Vagal nerve stimulation
A device (pulse generator)

implanted under the skin that sends

electrical pulses through the left

vagus nerve
- Little or no evidence of

cognitive impairment
- Invasive procedure requiring surgery

- Voice changes or hoarseness, neck pain, difficulty

swallowing, breathing problems

- Battery needs replacement every 7-10 years 
Overall, currently available treatments for depression are moderately efficacious, but side effects, treatment resistance and/or delay in therapeutic efficacy remain significant problems. Importantly, current medications are no more effective or different in mechanisms of action (targeting monoaminergic systems) than those introduced over 50 years ago.

\subsection{The promise and perils of ketamine}

A major breakthrough in the treatment of depression came in 2000 with ketamine at subanesthetic doses reported as a fast acting antidepressant drug. Ketamine is an US Food and Drug Administration (FDA)-approved anesthetic that has been used over the past 45 years in children and adults at doses of 1-3 mg/kg (Haas and Harper, 1992). It acts as a high affinity, noncompetitive N-methylD-aspartate (NMDA) receptor antagonist, binding to the open channel conformation of the NMDA receptor, at a site inside the calcium channel, which prevents the excitatory action of the neurotransmitter glutamate (Stahl, 2013b). In a 2000 study, using a randomized, placebo, crossover design (N=8), Berman and colleagues discovered that a low dose of ketamine $(0.5 \mathrm{mg} / \mathrm{kg})$ administered over a 40 -min intravenous (i.v.) infusion period produces rapid antidepressant response within $4 \mathrm{~h}$ of treatment, and that this response is sustained for at least $3 \mathrm{~d}$ (Berman et al., 2000). This finding was replicated in a second study in 2006 by Zarate and colleagues in patients with TRD $(\mathrm{N}=18)$, which reported an even more rapid antidepressant effect (within $2 \mathrm{~h}$ ) and a sustained effect for up to $7 \mathrm{~d}$ (Zarate et al., 2006). The primary efficacy measure was the Hamilton Depression Rating Scale (HDRS), with clinical response defined as a $50 \%$ or greater decrease in the HDRS score from baseline, and remission defined as an HDRS score of 7 or lower. Notably, $71 \%$ of the ketamine-treated patients met response criteria and $29 \%$ met remission criteria at $24 \mathrm{~h}$ after ketamine infusion; while no placebo-treated patients met response or remission criteria (Zarate et al., 2006). Since these two major landmark studies, there have been numerous other studies evaluating ketamine's fast acting antidepressant response and the results have been consistent with rapid reductions in depressive symptomatology. In the largest clinical trial of ketamine to date, patients with TRD were randomly assigned under double-blind conditions to receive a single i.v. infusion of 
ketamine or midazolam, an "active" placebo, in a 2:1 ratio ( $N=73$ ) (Murrough et al., 2013a). In this study, Murrough and colleagues demonstrated ketamine produced significantly higher response rates than midazolam at $24 \mathrm{~h}(64 \%$ and $28 \%$, respectively), with sustained effects for up to $7 \mathrm{~d}$ (Murrough et al., 2013a).

Along with decreases in several depression rating scales, ketamine produces rapid reductions specifically in suicidal ideation in depressed patients in open-label trials (DiazGranados et al., 2010b; Price et al., 2009), and in naturalistic studies and case reports in emergency department settings (Larkin and Beautrais, 2011; Zigman and Blier, 2013). Ketamine thus may offer an attractive intervention for acutely suicidal depressed patients. It also resolves depressive symptoms as add on therapy to lithium or valproic acid (VPA) for bipolar disorder (Cusin et al., 2012; Diazgranados et al., 2010a; Zarate et al., 2012). Most recently, it has been shown to reduce symptoms of post-traumatic stress disorder (PTSD), including a reduction in comorbid depressive symptoms, compared to the active placebo midazolam as early as $24 \mathrm{~h}$ post-infusion (Feder et al., 2014).

Despite these promising results, ketamine has significant limitations for widespread use as a treatment for depression. In a review of 205 ketamine infusions in 97 enrolled participants across 3 clinical trials at 2 centers, it was found that psychotomimetic symptoms, as measured by the Brief Psychiatric Rating Scale (BPRS), and dissociative symptoms, as measured by the Clinician-Administered Dissociative States Scale (CADSS), showed small, but significant elevations during the ketamine infusions (Wan et al., 2014). Though these changes all resolved within $4 \mathrm{~h}$ post-infusion (Wan et al., 2014), the psychotomimetic and dissociative effects are often part of a key predecessor to the mood elevating effects (Luckenbaugh et al., 2014; Sos et al., 2013). In addition, ketamine induced transient changes in hemodynamic measures in almost $30 \%$ of all participants, significantly elevating blood pressure and/or heart rate and resulting in ketamine discontinuation in 2 of the 205 patients (Wan et al., 2014). The most common side effects, as measured by the Systematic Assessment For Treatment Emergent Effects Self-Report Inventory (SAFTEE-SI) or the Patient-Rated Inventory of Side Effects 
(PRISE), were drowsiness, dizziness, poor coordination, blurred vision, and feeling strange or unreal (Wan et al., 2014).

In healthy subjects, along with psychotomimetic effects (Malhotra et al., 1996), sub-anesthetic doses of ketamine have been shown to induce transient dose-related cognitive impairments in memory, attention, and abstract reasoning (Newcomer et al., 1999; Perry et al., 2007). Of potentially greater concern are the relatively unknown risks of repeated ketamine administration. The reported negative consequences of prolonged use of high levels of ketamine include longer lasting cognitive impairments, deterioration in psychological well-being, psychotic symptoms, intense abdominal pain, ulcerative cystitis, and, in some instances, death (Morgan et al., 2012a). Studies examining the effects of repeated ketamine exposure on brain structure have also reported disruptions of white matter integrity and cortical atrophy in chronic abusers (Edward Roberts et al., 2014; Liao et al., 2011; Wang et al., 2013) and hippocampal atrophy in rodent models (Schobel et al., 2013).

In depressed patients, Murrough and colleagues reported an acute, selective impairment in memory recall 40 min after a single ketamine infusion (Murrough et al., 2013b). More extended followup in TRD patients given serial infusions of ketamine found no memory deficits at 4 weeks after the last infusion in one study (Shiroma et al., 2014) or 12 and 26 weeks in another study (Diamond et al., 2014). However, because there are no long-term studies of ketamine at low doses, particularly in mood disorder patients, its full safety profile remains unknown. Whether the frequency and the length of continuing treatment (e.g., maintenance) would cause temporary or permanent cognitive deficits requires further examination. Moreover, ketamine (or "Special K") is known to be a drug of abuse (Morgan et al., 2012b), raising additional concerns that repeated administration of the drug could have a liability for drug abuse.

\subsection{Ketamine: where do we go from here?}

Efforts thus far to overcome the side effect liabilities of ketamine have focused on: 1) utilizing alternate routes of administration and dosing to reduce the severity of the side effects, and 2) elucidating the critical mechanism(s) of the ketamine effects in an effort to identify or develop new compounds with 
better tolerability (e.g., compounds that target mechanisms downstream from the NMDA receptor). With regards to the first strategy, several routes of administration have been tested, including oral, sublingual, intramuscular and intranasal (Chilukuri et al., 2014; Cusin et al., 2012; Lapidus et al., 2014; Lara et al., 2013; Paslakis et al., 2010; Zanicotti et al., 2012). Though less side effects are seen, oftentimes higher doses or more frequent dosing are required with these different routes of administration. The second strategy has stimulated exciting new avenues of research in drug discovery that could benefit patients in the future and instigated numerous investigations into similar compounds, including dextromethorphan (DM), a potential novel antidepressant drug that is the focus of this dissertation.

\subsection{DM as a logical next step}

DM offers tantalizing promise for four main reasons: 1) it is available over-the-counter (OTC) as a cough suppressant for more than 50 years and has a wide margin of safety (Bem and Peck, 1992); 2) it has overlapping pharmacology with ketamine that is relevant for antidepressant actions (Lauterbach, 2012; Stahl, 2013a), suggesting rapid acting potential; 3) it binds to several protein targets which are similar to conventional medications as well as distinct from them, affording possibilities of efficacy in TRD (Lauterbach, 2012); and 4) in a recent retrospective study, DM produced antidepressant effects within a day or two in treatment-resistant bipolar depressed patients (Kelly and Lieberman, 2014).

We hypothesize that DM can activate sigma-1 receptors (a potential new target for antidepressant drugs) and AMPA (alpha-amino-3-hydroxy-5-methyl-4-isoxazolepropionic acid) receptors (a critical player in mediating ketamine's antidepressant effects downstream of NMDA receptor antagonism) as well as promote antidepressant-related neural adaptations (a final common pathway for conventional and fast acting antidepressants) to ultimately produce antidepressant effects.

Several preclinical studies have been carried out in this dissertation to begin evaluating the antidepressant potential of DM, with the following specific aims:

1. Determine the extent to which DM elicits antidepressant-like effects in vivo and evaluate the role of sigma-1 and AMPA receptors in these effects (chapters 3 and 4). 
2. Demonstrate the ability of DM to stimulate antidepressant-relevant neural adaptations in vitro and in vivo (chapters 5 and 6).

3. Since DM undergoes substantial first-pass metabolism, evaluate the impact of metabolism to determine whether the DM effects are likely mediated through itself or its major metabolites (chapters 8 and 9).

While chapter 2 details the pharmacokinetic and pharmacodynamic properties of DM and some important clinical considerations, the remaining chapters serve to offer supporting preclinical evidence for the antidepressant potential of DM. Uncovering DM's potential for safe and fast acting effects and/or efficacy in treatment-resistant individuals would improve the therapeutic armamentarium for depression and impact public health tremendously. 


\section{CHAPTER 2}

DM as a potential safe and effective antidepressant 


\subsection{History of DM}

DM has been a widely used non-opioid antitussive for over 50 years. It was first developed as one of two enantiomers of methorphan, a morphine derivative. DM is available in many OTC cough and cold preparations worldwide and does not possess the same central nervous system (CNS) pharmacodynamic effects of other opioids in humans (i.e., analgesia, respiratory depression or addiction) when taken at therapeutic doses (60-120 mg/day in divided doses) (Banken and Foster, 2008). At high doses (from 5 to over 10 times the label-specified maximum dosages), it acts as a dissociative agent similar to the NMDA antagonists ketamine and phencyclidine (PCP) (Banken and Foster, 2008; Romanelli and Smith, 2009; Schwartz, 2005). The levorotatory enantiomer of methorphan, levomethorphan, in contrast, is a low potency opiate analgesic (Stahl, 2013a); and its use is strictly controlled as a narcotic drug.

Over the past 20 years, accumulating evidence suggest that DM has both anticonvulsant and neuroprotective effects in numerous experimental models (Shin et al., 2011; Tortella et al., 1989; Werling et al., 2007b). Moreover, DM in combination with quinidine, a strong cytochrome P450 (CYP) 2D6 inhibitor, was approved by the FDA and the European Medicines Agency (EMA) in 2010 and 2013, respectively, for the treatment of pseudobulbar affect (PBA). PBA is characterized by sudden, unpredictable and involuntary episodes of crying, laughing, or other emotional displays that are exaggerated or incongruent with the mood and feelings of patients (Miller et al., 2011). It occurs secondary to a neurological disease or brain injury, with an estimated prevalence of up to $50 \%$ in amyotrophic lateral sclerosis and stroke, 39\% in Alzheimer's disease, 10-29\% in multiple sclerosis, 517\% in Parkinson's disease, and 5-11\% in traumatic brain injury (Miller et al., 2011). The combination dose of 20/10 $\mathrm{mg}$ for DM/quinidine is approved in the US, while a 20/10 $\mathrm{mg}$ and 30/10 $\mathrm{mg}$ dose is approved in Europe. Currently, DM is being used in clinical trials for a variety of CNS-related disorders, including stroke, traumatic brain injury, seizure, pain, methotrexate neurotoxicity, Parkinson's disease, autism, and importantly, depression (NIMH, 2015). 


\subsection{Pharmacokinetics and metabolism}

DM is commonly used as a probe drug for CYP2D6 metabolizer status since it undergoes extensive first-pass hepatic metabolism via O-demethylation to its active metabolite, dextrorphan (DX) (Capon et al., 1996; Yu and Haining, 2001). DM is also metabolized to a relatively inactive metabolite, 3methoxymorphinan (3-MM), via CYP3A4 N-demethylation (Yu and Haining, 2001). These DX and 3MM metabolites can both undergo further metabolism to another relatively inactive 3-hydroxymorphinan (3-HM) secondary metabolite via CYP3A4 and CYP2D6 demethylation, respectively (Yu and Haining, 2001). However, there is minimal free DX available for metabolism since this active metabolite is rapidly glucuronidated and excreted in urine (Pope et al., 2004). A summary of the metabolic pathway for DM is shown in Figure 1. DM and DX are both metabolized by CYP2D6, so it is useful to stratify pharmacokinetic parameters based on extensive ("normal") metabolizer (EM) or poor ("slow") metabolizer (PM) phenotype, and in some cases also intermediate metabolizer (IM) phenotype. In a study on 252 Americans, $84.3 \%$ were found to be EMs, $6.8 \%$ to be IMs, and $8.8 \%$ were PMs of DM (Woodworth et al., 1987). In a different study, EM subjects (N=6) given a single oral dose of $30 \mathrm{mg} D M$ have demonstrated a median half-life of $2.4 \mathrm{~h}$ with an oral bioavailability of $1-2 \%$, while PMs (N=6) have a median half-life of $19.1 \mathrm{~h}$ with an oral bioavailability of $80 \%$ (Capon et al., 1996). EM subjects have also demonstrated a DM median Cmax of $1.4 \mathrm{mg} / \mathrm{L}$ with an AUC of $9.0 \mathrm{mg} / \mathrm{L} \cdot \mathrm{h}$, while PMs have a median Cmax of $23.0 \mathrm{mg} / \mathrm{L}$ with an AUC of 1,362 $\mathrm{mg} / \mathrm{L} \cdot \mathrm{h}$ (Capon et al., 1996). After pretreatment with quinidine, a strong CYP2D6 inhibitor, EM subjects demonstrated a DM median half-life of 5.6 h, AUC of $383 \mathrm{mg} / \mathrm{L} \cdot \mathrm{h}$ and a Cmax of $24.9 \mathrm{mg} / \mathrm{L}$, which was not significantly different from the PM's Cmax (Capon et al., 1996).

\subsection{Pharmacodynamics profile}

DM has a complex and unique neuropharmacology that may underlie its apparent efficacy for indications besides cough suppression. Though DM is derived from levorphanol, a mu opioid agonist, it 
Figure 1. DM demethylation pathways catalyzed by CYP2D6 and CYP3A4. DX is formed by CYP2D6mediated O-demethylation of DM. N-demethylation of DM to 3-HM is favored over N-demethylation of DX given the relative $\mathrm{K}_{\mathrm{m}}$ values for the reactions and the ease with which DX is glucuronidated in vivo (adapted from (Blake et al., 2007)). DM, dextromethorphan; DX, dextrorphan; 3-MM, 3methoxymorphinan; 3-HM, 3-hydroxymorphinan.

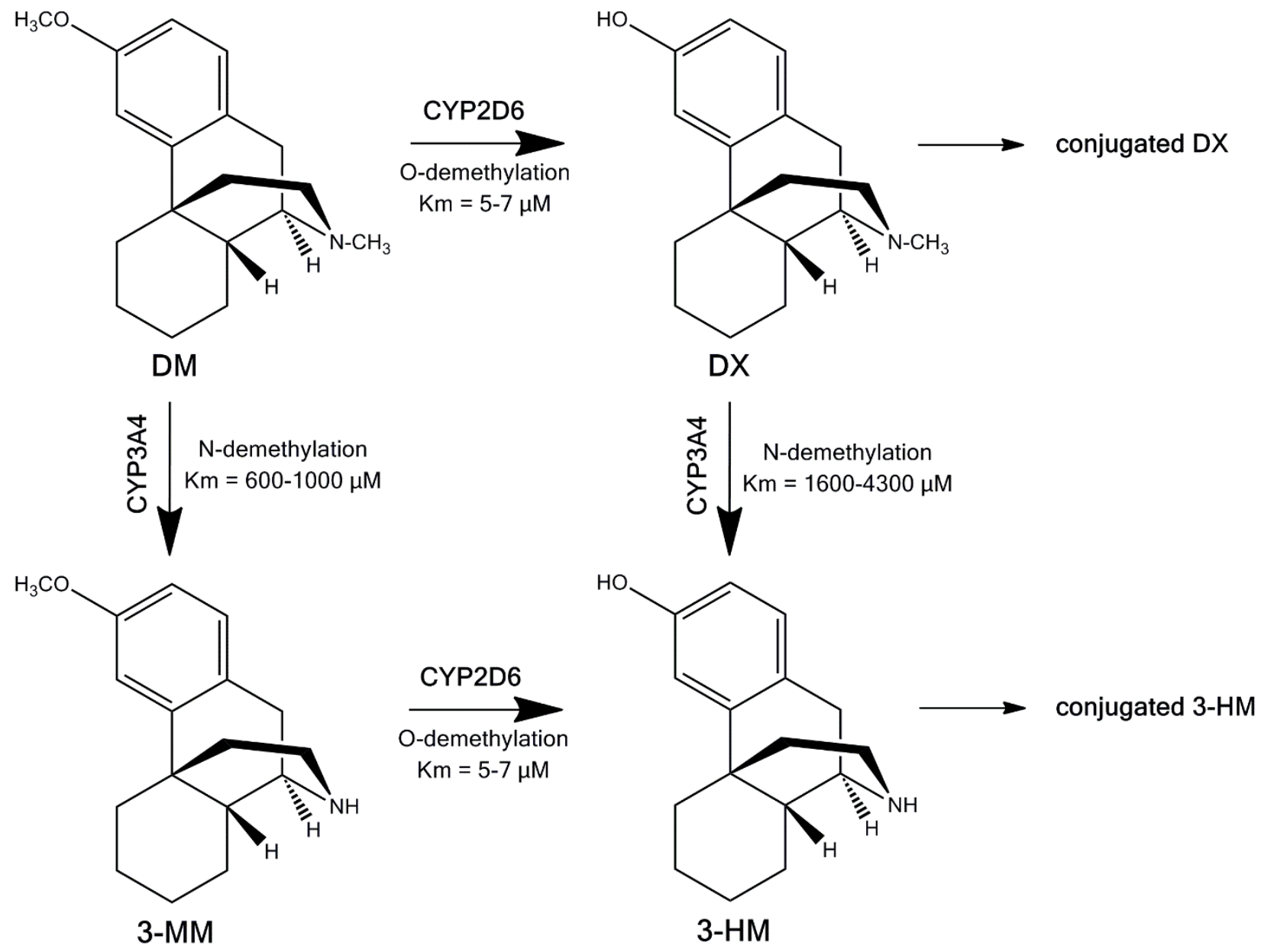


has no agonist activity at the classic opiate (mu, kappa, delta) receptors and does not carry the full range of CNS effects common to opioid agonists (e.g., analgesia, euphoria, respiratory depression), nor does it carry the same risk of dependence or addiction (Banken and Foster, 2008; Codd et al., 1995; Duman et al., 1988; Shin et al., 2011). DM binds to several other receptors and transporters in the brain, many with nanomolar to micromolar affinities (Table 2). DM is a well-established, uncompetitive, low-affinity NMDA receptor antagonist (Church et al., 1989; Church et al., 1985; Franklin and Murray, 1992; Netzer et al., 1993). Noteworthy, this low-affinity binding to NMDA receptors by DM is therapeutically useful because only low-affinity (vs. high-affinity) NMDA antagonists are generally tolerated by patients (Palmer, 2001). DM is also thought to act as an agonist at sigma-1 receptors (Nguyen et al., 2014), and an antagonist at nicotinic (alpha-3-beta-4, alpha-4-beta-2, and alpha-7) receptors (Damaj et al., 2005; Hernandez et al., 2000; Lee et al., 2006). It may also inhibit the serotonin transporter (SERT) and to a lesser extent the norepinephrine transporter (NET) (Codd et al., 1995) as well as voltage gated calcium channels (VGCC) (Carpenter et al., 1988; Kamel et al., 2008; Kim et al., 2001). DM's activity at other protein targets remains to be characterized.

\subsection{Proposed mechanisms of antidepressant action}

DM has multiple properties in common with known antidepressants and also unique properties, which may improve response in TRD cases (Lauterbach, 2012; Stahl, 2013b; Werling et al., 2007a). Noteworthy, DM has been postulated to have rapid acting antidepressant activity based on pharmacodynamic similarities to ketamine (Lauterbach, 2012). Unlike ketamine, however, DM is available OTC and has a high margin of safety. A side-by-side comparison of DM, ketamine and imipramine and their principal pharmacologic mechanisms of action are shown in Figure 2.

\subsubsection{Monoaminergic system}

The two original modern drugs used for the treatment of depression were the monoamine oxidase inhibitor (MAOI) iproniazid and the tricyclic antidepressant (TCA) imipramine. Both compounds were 
Table 2. Binding affinities $\left(\mathrm{K}_{\mathrm{i}}\right)$ for $\mathrm{DM}$ or $\mathrm{DX}$. $\mathrm{NC}=$ no competition at $1 \mu \mathrm{M}$.

\begin{tabular}{|c|c|c|c|c|c|}
\hline & DM & DX & Rat Tissue & Radioligand & Reference \\
\hline \multirow{4}{*}{ NMDA (PCP) } & $2120 \pm 84 \mathrm{nM}$ & $892 \pm 108 \mathrm{nM}$ & Hippocampus & {$\left[{ }^{3} \mathrm{H}\right] \mathrm{MK} 801+$ pentazocine } & (Werling et al., 2007a) \\
\hline & $7253 \pm 302 \mathrm{nM}$ & $906 \pm 77 \mathrm{nM}$ & Brain & {$\left[{ }^{3} \mathrm{H}\right] \mathrm{TCP}$} & (Chou et al., 1999) \\
\hline & $8945 \pm 867 \mathrm{nM}$ & $486 \pm 68 \mathrm{nM}$ & Brain & {$\left[{ }^{3} \mathrm{H}\right] \mathrm{TCP}$} & (Shin et al., 2007) \\
\hline & $8340 \pm 495 \mathrm{nM}$ & $696 \pm 87 \mathrm{nM}$ & Brain & {$\left[{ }^{3} \mathrm{H}\right] \mathrm{TCP}$} & (Kim et al., 2003b) \\
\hline NMDA & $\mathrm{NC}$ at $1 \mu \mathrm{M}$ & $\mathrm{NC}$ at $1 \mu \mathrm{M}$ & Hippocampus & {$\left[{ }^{3} \mathrm{H}\right] \mathrm{CGP} 39653$} & (Werling et al., 2007a) \\
\hline AMPA & $\mathrm{NC}$ at $1 \mu \mathrm{M}$ & $\mathrm{NC}$ at $1 \mu \mathrm{M}$ & Cortex & {$\left[{ }^{3} \mathrm{H}\right] \mathrm{AMPA}$} & (Werling et al., 2007a) \\
\hline Kainate & $\mathrm{NC}$ at $1 \mu \mathrm{M}$ & $\mathrm{NC}$ at $1 \mu \mathrm{M}$ & Forebrain & {$\left[{ }^{3} \mathrm{H}\right]$ Kainate } & (Werling et al., 2007a) \\
\hline Glycine & $\mathrm{NC}$ at $1 \mu \mathrm{M}$ & $\mathrm{NC}$ at $1 \mu \mathrm{M}$ & Brain & {$\left[{ }^{3} \mathrm{H}\right]$ Strychnine } & (Werling et al., 2007a) \\
\hline \multirow{13}{*}{ Sigma-1 } & $150 \pm 47 \mathrm{nM}$ & $118 \pm 77 \mathrm{nM}$ & Cerebellum & $\begin{array}{l}{\left[{ }^{3} \mathrm{H}\right](+) \text {-Pentazocine }+} \\
\text { Lu28-179 }\end{array}$ & (Werling et al., 2007a) \\
\hline & $196 \pm 74 \mathrm{nM}$ & -- & Pons & $\begin{array}{l}{\left[{ }^{3} \mathrm{H}\right](+) \text {-Pentazocine }+} \\
\text { Lu28-179 }\end{array}$ & (Werling et al., 2007a) \\
\hline & $138 \pm 48 \mathrm{nM}$ & $351 \pm 39 n M$ & Brain & $\begin{array}{l}{\left[{ }^{3} \mathrm{H}\right](+)-S K F 10,047+} \\
\text { MK801 }\end{array}$ & (Nam et al., 2012) \\
\hline & $161 \pm 57 \mathrm{nM}$ & $481 \pm 64 \mathrm{nM}$ & Testes & $\begin{array}{l}{\left[{ }^{3} \mathrm{H}\right](+)-\mathrm{SKF} 10,047+} \\
\mathrm{MK} 801\end{array}$ & (Nam et al., 2012) \\
\hline & $142 \pm 38 \mathrm{nM}$ & $344 \pm 47 \mathrm{nM}$ & Brain & $\begin{array}{l}{\left[{ }^{3} \mathrm{H}\right](+)-\mathrm{SKF} 10,047+} \\
\mathrm{MK} 801\end{array}$ & (Shin et al., 2007) \\
\hline & $180 \pm 28 \mathrm{nM}$ & $294 \pm 36 \mathrm{nM}$ & Brain & $\begin{array}{l}{\left[{ }^{3} \mathrm{H}\right](+)-S K F 10,047+} \\
\mathrm{MK} 801\end{array}$ & (Kim et al., 2003b) \\
\hline & $403 \pm 22 \mathrm{nM}$ & -- & Brain & {$\left[{ }^{3} \mathrm{H}\right](+)$-Pentazocine } & (Fishback et al., 2012) \\
\hline & $214 \pm 15 \mathrm{nM}$ & -- & Liver & {$\left[{ }^{3} \mathrm{H}\right](+)$-Pentazocine } & (Fishback et al., 2012) \\
\hline & $205 \pm 42 \mathrm{nM}$ & $144 \pm 37 \mathrm{nM}$ & Brain & {$\left[{ }^{3} \mathrm{H}\right](+)-\mathrm{SKF} 10,047$} & (Chou et al., 1999) \\
\hline & $652 \pm 33 \mathrm{nM}$ & -- & Brain & {$\left[{ }^{3} \mathrm{H}\right](+)$-Pentazocine } & (Klouz et al., 2002) \\
\hline & $>10,000 \mathrm{nM}$ & -- & Brain Mitochondria & {$\left[{ }^{3} \mathrm{H}\right](+)$-Pentazocine } & (Klouz et al., 2002) \\
\hline & $217 \pm 17 \mathrm{nM}$ & -- & Liver Mitochondria & {$\left[{ }^{3} \mathrm{H}\right](+)$-Pentazocine } & (Klouz et al., 2002) \\
\hline & $528 \pm 6 \mathrm{nM}$ & -- & Liver Microsomes & {$\left[{ }^{3} \mathrm{H}\right](+)$-Pentazocine } & (Klouz et al., 2002) \\
\hline \multirow{4}{*}{ Sigma-2 } & $\mathrm{NC}$ at $1 \mu \mathrm{M}$ & $\mathrm{NC}$ at $1 \mu \mathrm{M}$ & Cerebellum & {$\left[{ }^{3} \mathrm{H}\right] \mathrm{DTG}+\mathrm{DuP734}$} & (Werling et al., 2007a) \\
\hline & $19,976 \pm 2144 \mathrm{nM}$ & $12,899 \pm 2,015 \mathrm{nM}$ & Brain & {$\left[{ }^{3} \mathrm{H}\right] \mathrm{DTG}+(+)$ SKF10,047 } & (Nam et al., 2012) \\
\hline & $22,864 \pm 1917 \mathrm{nM}$ & $15,582 \pm 2114 \mathrm{nM}$ & Testes & {$\left[{ }^{3} \mathrm{H}\right] \mathrm{DTG}+(+) \mathrm{SKF} 10,047$} & (Nam et al., 2012) \\
\hline & $16,873 \pm 2234 \mathrm{nM}$ & $12,987 \pm 1975 \mathrm{nM}$ & Brain & {$\left[{ }^{3} \mathrm{H}\right] \mathrm{DTG}+(+)$ SKF10,047 } & (Shin et al., 2007) \\
\hline
\end{tabular}




\begin{tabular}{|c|c|c|c|c|c|}
\hline & $12,079 \pm 1638 \mathrm{nM}$ & $11,457 \pm 1437 \mathrm{nM}$ & Brain & {$\left[{ }^{3} \mathrm{H}\right] \mathrm{DTG}+(+) \mathrm{SKF} 10,047$} & (Kim et al., 2003b) \\
\hline & $>10,000 \mathrm{nM}$ & -- & Brain & {$\left[{ }^{3} \mathrm{H}\right] \mathrm{DTG}+$ pentazocine } & (Fishback et al., 2012) \\
\hline & $>10,000 \mathrm{nM}$ & -- & Liver & {$\left[{ }^{3} \mathrm{H}\right] \mathrm{DTG}+$ pentazocine } & (Fishback et al., 2012) \\
\hline & $11,060 \pm 1320 \mathrm{nM}$ & $11,325 \pm 1395 \mathrm{nM}$ & Brain & {$\left[{ }^{3} \mathrm{H}\right] \mathrm{DTG}$} & (Chou et al., 1999) \\
\hline \multirow[b]{2}{*}{ SERT } & $40 \pm 7 \mathrm{nM}$ & $484 \pm 116 \mathrm{nM}$ & Hippocampus & {$\left[{ }^{3} \mathrm{H}\right]$ Paroxetine } & (Werling et al., 2007a) \\
\hline & $23 \mathrm{nM}^{*}$ & $401 \mathrm{nM}^{*}$ & Cortex & $\begin{array}{l}{\left[{ }^{3} \mathrm{H}\right] 5-\mathrm{HT}} \\
\text { *uptake rather than binding } \\
\text { assay }\end{array}$ & (Codd et al., 1995) \\
\hline 5-HT1A & $\mathrm{NC}$ at $1 \mu \mathrm{M}$ & $\mathrm{NC}$ at $1 \mu \mathrm{M}$ & Hippocampus & {$\left[{ }^{3} \mathrm{H}\right] 8-\mathrm{OH}-\mathrm{DPAT}$} & (Werling et al., 2007a) \\
\hline 5-HT1B/D & $61 \%$ at $1 \mu \mathrm{M}$ & $54 \%$ at $1 \mu \mathrm{M}$ & Cortex & {$\left[{ }^{3} \mathrm{H}\right] \mathrm{GR} 125,743$} & (Werling et al., 2007a) \\
\hline 5-HT2 & $\mathrm{NC}$ at $1 \mu \mathrm{M}$ & $\mathrm{NC}$ at $1 \mu \mathrm{M}$ & Hippocampus & {$\left[{ }^{3} \mathrm{H}\right]$ Ketanserine } & (Werling et al., 2007a) \\
\hline \multirow[t]{2}{*}{ NET } & $\mathrm{NC}$ at $1 \mu \mathrm{M}$ & $\mathrm{NC}$ at $1 \mu \mathrm{M}$ & Cortex & {$\left[{ }^{3} \mathrm{H}\right]$ Nisozetine } & (Werling et al., 2007a) \\
\hline & $240 \mathrm{nM}^{*}$ & $340 \mathrm{nM}^{*}$ & Medulla/Pons & $\begin{array}{l}{\left[{ }^{3} \mathrm{H}\right] \mathrm{NE}} \\
\text { *uptake rather than binding } \\
\text { assay }\end{array}$ & (Codd et al., 1995) \\
\hline $\begin{array}{l}\text { Alpha-1 } \\
\text { adrenergic }\end{array}$ & $\mathrm{NC}$ at $1 \mu \mathrm{M}$ & $\mathrm{NC}$ at $1 \mu \mathrm{M}$ & Hippocampus & {$\left[{ }^{3} \mathrm{H}\right]$ Prazosin } & (Werling et al., 2007a) \\
\hline $\begin{array}{l}\text { Alpa-2 } \\
\text { adrenergic }\end{array}$ & $60 \%$ at $1 \mu \mathrm{M}$ & $\mathrm{NC}$ at $1 \mu \mathrm{M}$ & Hippocampus & {$\left[{ }^{3} \mathrm{H}\right]$ Yohimbine } & (Werling et al., 2007a) \\
\hline Beta adrenergic & $\mathrm{NC}$ at $1 \mu \mathrm{M}$ & $35 \%$ at $1 \mu \mathrm{M}$ & Cortex & {$\left[{ }^{3} \mathrm{H}\right]$ Dihydroalprenolol } & (Werling et al., 2007a) \\
\hline Mu opioid & $1280 \mathrm{nM}$ & $420 \mathrm{nM}$ & Forebrain & {$\left[{ }^{3} \mathrm{H}\right] \mathrm{DAMGO}$} & (Codd et al., 1995) \\
\hline Kappa opioid & $7000 \mathrm{nM}$ & $5950 \mathrm{nM}$ & Forebrain & {$\left[{ }^{3} \mathrm{H}\right] \mathrm{U} 69,593$} & (Codd et al., 1995) \\
\hline Delta opioid & $11,500 \mathrm{nM}$ & $34,700 \mathrm{nM}$ & Forebrain & {$\left[{ }^{3} \mathrm{H}\right] \mathrm{DPDPE}$} & (Codd et al., 1995) \\
\hline Nicotinic & $\mathrm{NC}$ at $1 \mu \mathrm{M}$ & $\mathrm{NC}$ at $1 \mu \mathrm{M}$ & Forebrain & {$\left[{ }^{3} \mathrm{H}\right]$ Epibatidine } & (Werling et al., 2007a) \\
\hline \multirow{3}{*}{$\begin{array}{l}\text { Alpha-3-beta-4 } \\
\text { nicotinic }\end{array}$} & $<20 \%$ at $100 \mu \mathrm{M}$ & $<20 \%$ at $100 \mu \mathrm{M}$ & $\begin{array}{l}\text { Transfected HEK-293 } \\
\text { cells }\end{array}$ & {$\left[{ }^{3} \mathrm{H}\right]$ Epibatidine } & (Hernandez et al., 2000) \\
\hline & $8.9 \pm 1.1 \mu \mathrm{M}^{*}$ & $29.6 \pm 5.7 \mu \mathrm{M}^{*}$ & $\begin{array}{l}\text { Transfected HEK-293 } \\
\text { cells }\end{array}$ & $\begin{array}{l}* \mathrm{IC}_{50} \text { values }(50 \% \text { of } \\
\text { nicotine-stimulated }{ }^{86} \mathrm{Rb}+ \\
\text { efflux })\end{array}$ & (Hernandez et al., 2000) \\
\hline & $0.7 \pm 0.1 \mu \mathrm{M}^{*}$ & $1.3 \pm 0.1 \mu \mathrm{M}^{*}$ & $\begin{array}{l}\text { Transfected Xenopus } \\
\text { laevis oocytes }\end{array}$ & $\begin{array}{l}* \mathrm{IC}_{50} \text { values }(50 \% \text { of } \\
\text { acetylcholine-stimulated } \\
\text { nicotinic current) }\end{array}$ & (Damaj et al., 2005) \\
\hline
\end{tabular}




\begin{tabular}{|l|l|l|l|l|l|}
\hline $\begin{array}{l}\text { Alpha-4-beta-2 } \\
\text { nicotinic }\end{array}$ & $3.9 \pm 0.2 \mu \mathrm{M}^{*}$ & $3.0 \pm 0.5 \mu \mathrm{M}^{*}$ & $\begin{array}{l}\text { Transfected Xenopus } \\
\text { laevis oocytes }\end{array}$ & $\begin{array}{l}\text { *IC } 50 \text { values (50\% of } \\
\text { acetylcholine-stimulated } \\
\text { nicotinic current) }\end{array}$ & (Damaj et al., 2005) \\
\hline $\begin{array}{l}\text { Alpha-7 } \\
\text { nicotinic }\end{array}$ & $2.5 \pm 0.2 \mu \mathrm{M}^{*}$ & $4.3 \pm 0.2 \mu \mathrm{M}^{*}$ & $\begin{array}{l}\text { Transfected Xenopus } \\
\text { laevis oocytes }\end{array}$ & $\begin{array}{l}\text { *IC } \mathrm{I}_{50} \text { values (50\% of } \\
\text { acetylcholine-stimulated } \\
\text { nicotinic current) }\end{array}$ & (Damaj et al., 2005) \\
\hline Histamine-1 & $\mathrm{NC}$ at $1 \mu \mathrm{M}$ & $95 \%$ at $1 \mu \mathrm{M}$ & Cortex & {$\left[{ }^{3} \mathrm{H}\right]$ Mepyramine } & (Werling et al., 2007a) \\
\hline Histamine-2 & $\mathrm{NC}$ at $1 \mu \mathrm{M}$ & $\mathrm{NC}$ at $1 \mu \mathrm{M}$ & Cortex & {$\left[{ }^{3} \mathrm{H}\right]$ Cimetidine } & (Werling et al., 2007a) \\
\hline Dopamine-1 & $\mathrm{NC}$ at $1 \mu \mathrm{M}$ & $\mathrm{NC}$ at $1 \mu \mathrm{M}$ & Striatum & {$\left[{ }^{3} \mathrm{H}\right] \mathrm{SCH} 23390$} & (Werling et al., 2007a) \\
\hline Dopamine-2 & $\mathrm{NC}$ at $1 \mu \mathrm{M}$ & $\mathrm{NC}$ at $1 \mu \mathrm{M}$ & Striatum & [ $\left.{ }^{3} \mathrm{H}\right]$ Spiroperidol & (Werling et al., 2007a) \\
\hline DAT & $\mathrm{NC}$ at $1 \mu \mathrm{M}$ & $\mathrm{NC}$ at $1 \mu \mathrm{M}$ & Striatum & [ $\left.{ }^{3} \mathrm{H}\right] \mathrm{WIN35,428}$ & (Werling et al., 2007a) \\
\hline GABAA & $\mathrm{NC}$ at $1 \mu \mathrm{M}$ & $\mathrm{NC}$ at $1 \mu \mathrm{M}$ & Cortex & {$\left[{ }^{3} \mathrm{H}\right]$ Muscimol } & (Werling et al., 2007a) \\
\hline GABAB & $\mathrm{NC}$ at $1 \mu \mathrm{M}$ & $\mathrm{NC}$ at $1 \mu \mathrm{M}$ & Cortex & {$\left[{ }^{3} \mathrm{H}\right]$ Baclofen } & (Werling et al., 2007a) \\
\hline L-type Ca ${ }^{2+}$ & $\mathrm{NC}$ at $1 \mu \mathrm{M}$ & $\mathrm{NC}$ at $1 \mu \mathrm{M}$ & Striatum & {$\left[{ }^{3} \mathrm{H}\right] \mathrm{PN} 200-100$} & (Werling et al., 2007a) \\
\hline
\end{tabular}


Figure 2. Comparison of pharmacologic actions of DM, ketamine, and imipramine. The binding properties of the compounds are represented graphically and semi-quantitatively. Each drug is shown as a blue sphere, with its most potent binding properties along the outer edge of the sphere. Additionally each drug has a series of colored boxes associated with it. Each colored box represents a different binding property, and binding affinity is indicated by the size of the box and the number of plus signs. Within the colored box series for each drug, large boxes with more plus signs represent higher binding affinity, while smaller boxes with fewer plus signs represent lower binding affinity. The series of boxes associated with each drug are arranged such that the size and positioning of a box reflect the biding potency for a particular receptor (adapted from (Stahl, 2013a)). DM, dextromethorphan; NMDA, N-methyl-D-aspartate; SERT, serotonin transporter; NET, norepinephrine transporter; DAT, dopamine transporter; D-2, dopamine D2.
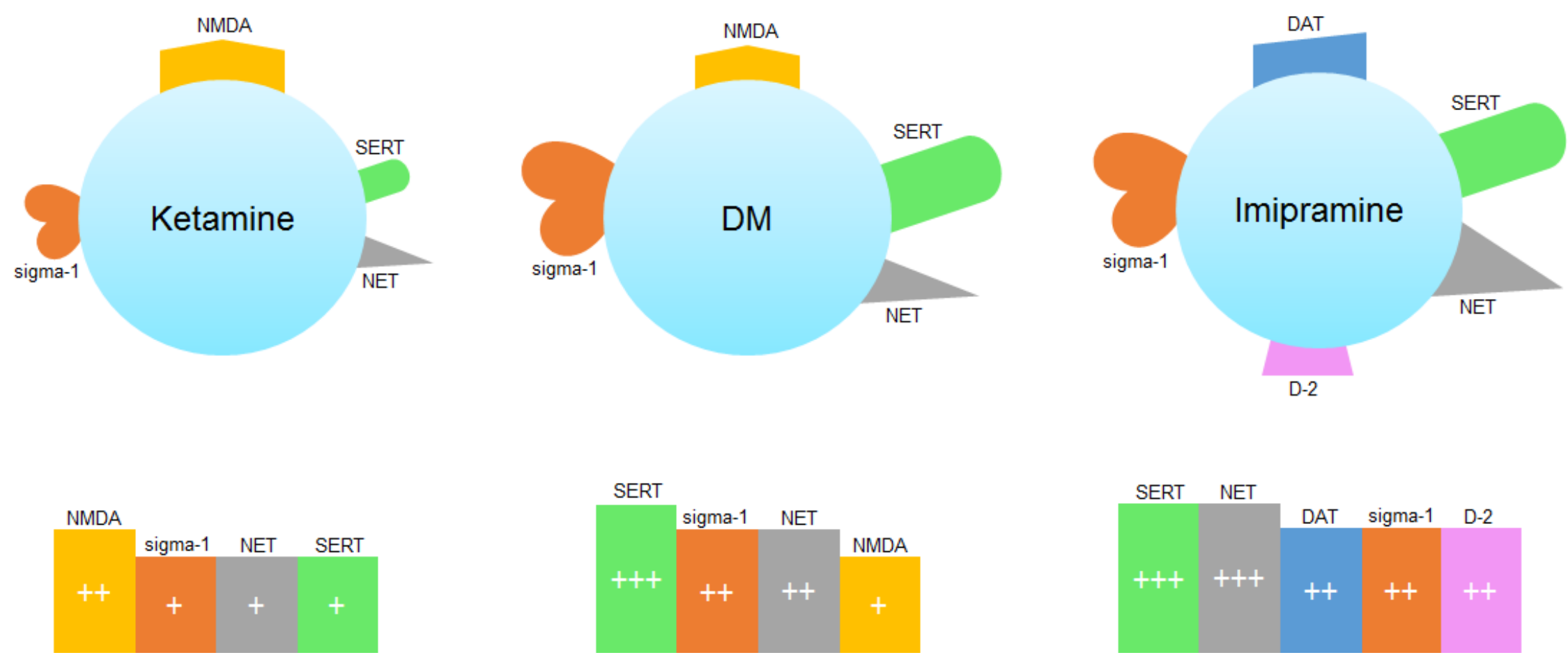
discovered serendipitously in the 1950s, with iproniazid being developed as a treatment for tuberculosis and imipramine as an antihistamine but tested for schizophrenia due to the success of chlorpromazine (Slattery et al., 2004). The discovery of the mechanisms of actions of these compounds led to the monoamine-deficient hypothesis of depression, which proposes that the underlying biological or neuroanatomical basis for depression is a depletion of central noradrenergic and/or serotonergic systems and that increasing levels of these monoamines would correct the deficit and restore normal function in depressed patients (Slattery et al., 2004).

DM binds to SERT with high affinity $\left(\mathrm{K}_{\mathrm{i}}=40 \mathrm{nM}\right)$ (Werling et al., 2007a), and has been reported to inhibit synaptosomal uptake of serotonin (5-HT) (Codd et al., 1995). Moreover, there is electrophysiological evidence that it can increase 5-HT release in rat brainstem slices (Kamei, Mori et al. 1992), which may be mediated through its interaction at the SERT and 5-HT1B/D receptor. DM also binds to NET, though much more weakly (>1 $1 \mu \mathrm{M}$ ) (Werling et al., 2007a), and has been shown to modulate norepinephrine (NE) reuptake (Codd et al., 1995). The ability of DM to increase the levels of these key monoamines suggest it would elicit some antidepressant effects clinically. Indeed, the current antidepressant therapies, which elevate the levels of these monoamines in the synaptic cleft, have proved very successful and remain some of the most widely prescribed drugs in the US (Statistics, 2014).

However, a discrepancy exists in the monoamine hypothesis of depression: while medications can increase the amount of these neurotransmitters in the synaptic cleft within hours, clinical effects of these agents are observed after a considerable delay of continuous daily administration. In addition, there are a substantial number of patients who remain resistant to the current therapies, indicating that these neurotransmitters may not be the only key molecular players responsible for resolving depression. Due to the incomplete nature of the monoamine theories of depression, research has attempted to determine other systems that may be involved in depression and on the molecular events downstream of an antidepressant's direct actions on the monoamines. From this, several new theories about the pathophysiology of depression and the action of antidepressant medications have arisen, and new families of potential targets for novel antidepressant therapies have been identified. 


\subsubsection{Sigma-1 receptor}

One potential protein target that has been implicated for existing and novel antidepressant drugs is the sigma-1 receptor (Fishback et al., 2010). Through its high affinity binding to and activation of sigma-1 receptors (Lauterbach, 2012; Werling et al., 2007a), DM may thus also produce antidepressant effects. Noteworthy, sigma-1 receptor agonists may facilitate a more rapid onset of efficacy than FDA-approved antidepressants (Hayashi and Su, 2008).

Sigma-1 receptors are highly conserved 223 amino acid proteins expressed on the mitochondrialassociated endoplasmic reticulum membrane (MAM) and can translocate between different cellular compartments in response to ligand binding (Hayashi and $\mathrm{Su}, 2007$ ). In addition, sigma-1 receptors appear to operate primarily via protein-protein interactions to modulate the activity of various ion channels and signaling molecules, including inositol triphosphates, protein kinases, and calcium (Hayashi and $\mathrm{Su}, 2007$; $\mathrm{Su}$ et al., 2010). In adult males with MDD, there has been a report of a decreased plasma sigma-1 receptor concentration compared to healthy controls (Takebayashi, 2007). In addition, another study demonstrated an increase in plasma sigma-1 receptor concentration following antidepressant treatment in patients with late-life MDD (Shimizu et al., 2013). However, the latter study did not find a correlation between an increase in plasma sigma-1 levels and reductions in depressive symptoms as measured by the HDRS, although this may be due to the small sample size (N=12) (Shimizu et al., 2013). In preclinical studies, knockout of sigma-1 receptors in mice resulted in a depressive-like phenotype (Sabino et al., 2009). These studies suggest that a decrease in sigma-1 receptor function may play some role in the pathophysiology of depression.

Relevant to antidepressant actions, currently marketed antidepressant drugs, such as TCAs, MAOIs, selective serotonin reuptake inhibitors (SSRIs), and newer generations of antidepressant drugs, bind to these receptors (Fishback et al., 2010). Earlier studies also demonstrate that sigma-1 receptor agonists can modulate the activities of neurotransmitter systems, signaling pathways and brain regions implicated in the pathophysiology of depression (Fishback et al., 2010). The potential clinical relevance of these observations is further supported by reports that sigma-1 agonists produce antidepressant effects 
in experimental animals and humans (Matsuno et al., 1996; Skuza, 2003; Skuza and Rogoz, 2002; Ukai et al., 1998; Volz and Stoll, 2004; Wang et al., 2007). Notably, the sigma-1 receptor agonist igmesine hydrochloride proved to be as effective an antidepressant as the well-established SSRI fluoxetine in some clinical trials, though not in all cases (Fishback et al., 2010; Volz and Stoll, 2004).

Specifically related to fast acting effects, sigma-1 receptor agonists such as (+)-pentazocine and SA4503 (1-[2-(3,4-Dimethoxyphenyl)ethyl]-4-(3-phenylpropyl)piperazine) can enhance serotonergic neuronal firing in the rat dorsal raphe nucleus after only two days of treatment, compared to the two weeks of treatment that is typically required of conventional antidepressant drugs (Bermack and Debonnel, 2001; Lucas et al., 2008). Moreover, SA4503 also enhances cell proliferation in the rat hippocampus after only 3 days of treatment, compared to the 2-3 weeks required for classical antidepressants (Lucas et al., 2008). It should be also noted that sigma receptor modulation has been linked to neural plasticity, which is thought to be final common pathway of different antidepressant therapies (see section 2.3.4) (Duman, 2014a; Nestler et al., 2002a). For instance, several studies have shown sigma receptor mediation of nerve growth factor (NGF)-induced neurite sprouting (Ishima et al., 2008; Nishimura et al., 2008; Robson et al., 2012; Takebayashi et al., 2002), suggesting that certain antidepressants may facilitate neuronal sprouting in the brain via these receptors.

\subsubsection{Glutamatergic system}

DM may also elicit antidepressant effects through modulation of glutamatergic function, which is increasingly implicated in the pathogenesis and pharmacology of depression (Hashimoto, 2011; Niciu et al., 2013). Similar to ketamine, DM is an NMDA receptor antagonist, which is thought to be the primary mechanism by which ketamine produces its rapid acting effects (Monteggia and Zarate, 2015). The NMDA receptors exist in vivo as tetrameric complexes comprising proteins from two families of homologous subunits, designated NR1 and NR2(A-D) (Feyissa et al., 2009). In patients with MDD, a significant reduction in NR2A and NR2B, but not NR1 subunit expression was found in the prefrontal cortex (PFC), a region that has long been implicated in the pathophysiology of depression (Feyissa et al., 
2009). Interestingly, sigma-1 receptor agonists have been shown to alter NMDA receptor activity in rat retinal slice preparations (Zhang et al., 2011) as well as upregulate NMDA receptor expression (specifically NR2A and NR2B, but not NR1) in the rat hippocampus (Pabba et al., 2014; Zhang et al., 2011). Whether DM may induce changes in the activity or level of specific NMDA subunits in human brain regions expressing sigma-1 receptors remains to be determined.

Along with NMDA receptors, AMPA receptors also appear to be critical for antidepressant response, including rapid acting effects (Alt et al., 2006; Bleakman et al., 2007). AMPA receptors are tetrameric ionotropic receptors assembled from the four subunits GluA1, GluA2, GluA3 and GluA4, of which several isoforms with differing activation kinetics exist (Fleming and England, 2010). Several studies showed a reduction in AMPA receptor mRNA and protein levels in patients with depression and in animals after stress induction or corticosterone treatment (Freudenberg et al., 2015). However, in other studies increases in AMPA receptors were shown, suggesting a more complex contribution of these receptors to the pathophysiology of depression (Freudenberg et al., 2015). This complexity might in part be due to differences in the contribution of the different AMPA receptor subunits (Freudenberg et al., 2015).

Pharmacologically, AMPA receptor potentiators, which increase receptor function by altering receptor kinetics (e.g., decrease receptor desensitization or deactivation), can produce antidepressant-like effects on their own as well as in synergy with other antidepressants in several animal models of depression (Farley et al., 2010; Knapp et al., 2002; Li et al., 2001). More recently, AMPA itself has been shown to produce antidepressant-like behaviors and can potentiate the antidepressant-like actions of ketamine (Akinfiresoye and Tizabi, 2013). These data suggests that AMPA receptors may work independently and/or together with other pathways to elicit antidepressant-like effects. In fact, AMPA receptors may be a common downstream pathway for known antidepressants that act initially on entirely different molecular targets (Bleakman et al., 2007). For instance, treatments with diverse antidepressants have been shown to modulate AMPA receptor function and/or expression, including the SSRIs fluoxetine and paroxetine, NE reuptake inhibitor reboxetine, TCA desipramine, and ketamine (Barbon et al., 2011; 
Barbon et al., 2006; Du et al., 2007; Li et al., 2010; Maeng et al., 2008; Martinez-Turrillas et al., 2005, 2007; Martinez-Turrillas et al., 2002; Svenningsson et al., 2002; Zhou et al., 2013).

Importantly, blockade of AMPA receptors with an antagonist has been shown to prevent the antidepressant effects of the rapid acting antidepressants ketamine (Autry et al., 2011; Koike et al., 2011; Maeng et al., 2008) and the more recently discovered scopolamine (Voleti et al., 2013), but failed to block that of conventional antidepressants such as imipramine in some instances (Maeng et al., 2008; Wolak et al., 2013), implying that AMPA receptors are critical for fast acting antidepressant effects. In addition, Knapp et al. reported that an AMPA receptor potentiator produces a more rapid onset of action than the conventional antidepressant fluoxetine in the reduction of the submissive behavior paradigm (Knapp et al., 2002), a recently developed rodent model for assessing antidepressant drug activity (Malatynska et al., 2002; Malatynska et al., 2005).

\subsubsection{BDNF and neural plasticity}

As mentioned above, neural plasticity is hypothesized to be a final common pathway of different antidepressant therapies and may explain the delay in efficacy with conventional antidepressants (Duman, 2014a; Nestler et al., 2002a). An important player thought to be involved in driving the neural adaptations is brain-derived neurotrophic factor (BDNF) (Castren and Rantamaki, 2010). BDNF protects neurons from injury, at least in part, by inhibiting apoptosis and by stimulating sprouting and neuronal reorganization (Castren and Rantamaki, 2010). Although BDNF is highly concentrated in the nervous system, it is also found in the serum of humans and other mammals, where its function is poorly understood (Karege et al., 2005).

Several studies have shown that serum BDNF levels are reduced in unipolar and bipolar depression and can be normalized by successful treatment (Castren and Rantamaki, 2010; Grande et al., 2010). In a double-blind study of bipolar disorder patients (type I or II) given placebo or DM (30 or 60 $\mathrm{mg} /$ day), in combination with VPA for 12 weeks, participants had lower levels of plasma BDNF compared to healthy controls at baseline (Chen et al., 2014). Subsequent treatment with DM (60 mg/day) 
plus VPA produced a small increase in plasma BDNF levels from baseline to 12 weeks, and the increase was significantly higher than the placebo plus VPA group (Chen et al., 2014). There was also a trend toward increased BDNF found in the DM group compared to baseline. Although this small increase did not correlate with measured improvements of clinical symptoms in this study, it may still have benefits not measured, like decreased duration of depressive symptoms (Chen et al., 2014). It remains unclear however whether serum BDNF levels might reflect or contribute to the BDNF levels in the brain, as BDNF is not able to readily cross the blood-brain barrier (Castren and Rantamaki, 2010). Moreover, despite the name, BDNF is also expressed at relatively high levels in peripheral tissues, including lung, heart, and spleen; thus, BDNF in serum is likely to be derived from these tissues as well as from brain (Schmidt and Duman, 2010).

Post-mortem studies have reported reductions in BDNF and tropomyosin receptor kinase B (TrkB) expression in the hippocampus and PFC of MDD patients and depressed suicides. For instance, a reduction in BDNF levels was found in the PFC and hippocampus of suicide victims who were depressed relative to matched controls or patients taking an antidepressant at the time of death (Dwivedi et al., 2001); treatments with antidepressants have led to an upregulation of BDNF in the hippocampus of MDD patients at the time of death compared with antidepressant-untreated subjects (Chen et al., 2001).

Noteworthy, activation of AMPA receptors has been shown to increase rat hippocampal BDNF mRNA expression within a few hours of treatment (Mackowiak et al., 2002) compared to the chronic dosing that is typically required for conventional antidepressants (Duman and Monteggia, 2006). In contrast to conventional antidepressants that promote the transcriptional upregulation of BDNF, activation of sigma-1 receptors has been shown to potentiate post-translational processing (i.e., the conversion of pro-BDNF to BDNF) without affecting the mRNA expression of BDNF, which may provide a novel therapeutic opportunity for the treatment of depression (Fujimoto et al., 2012). Through activation of sigma-1 receptors, DM may also promote an increase in the expression or activity of other trophic factors such as NGF (Fishback et al., 2010). It is possible that DM, through these actions, may facilitate neural 
adaptations faster than conventional antidepressants and thereby induce more rapid therapeutic effects (Figure 3).

\subsubsection{Other mechanisms}

Other mechanisms may also contribute to the antidepressant effects of DM, including possible activity at 5-HT1B/D, alpha-2 noradrenergic, and nicotinic receptors (Damaj et al., 2005; Hernandez et al., 2000; Werling et al., 2007a). The time lag in current SSRI antidepressant medications is believed, at least in part, to be due to desensitization of presynaptic 5-HT1A and 5-HT1B receptor subtypes, which occurs over 2-4 weeks (Gardier et al., 1996; Rutter et al., 1994). In particular, 5-HT1B receptors function as both presynaptic autoreceptors and postsynaptic heteroreceptors (Moret and Briley, 2000). 5-HT1B autoreceptors are located presynaptically on serotonergic neurons and are thought to be involved in a feedback mechanism that inhibits 5-HT neuronal firing and release. In contrast, 5-HT1B heteroreceptors are located on non-serotonergic neurons, including glutamatergic, GABA (gamma-aminobutyric acid)ergic, dopaminergic, noradrenergic and cholinergic neurons, and may exhibit inhibitory activity (Moret and Briley, 2000). Preclinical studies have shown that application of selective 5-HT1A agonists alone and with antidepressants can produce antidepressant-like effects (Ruf and Bhagwagar, 2009). Interestingly, in male rhesus monkeys, ketamine significantly increased 5-HT1B receptor binding in the nucleus accumbens and ventral pallidum, whereas it significantly reduced SERT binding in these brain regions (Yamanaka et al., 2014). Pretreatment with the AMPA receptor antagonist, NBQX (2,3-dihydroxy-6nitro-7-sulfamoylbenzo[f]quinoxaline), blocked the action of ketamine on the 5-HT1B receptor but not SERT binding, which suggests the involvement of AMPA receptor activation in ketamine-induced alterations of 5-HT1B receptor binding (Yamanaka et al., 2014). The authors noted that because NBQX is known to block the antidepressant effect of ketamine in rodents, alterations in serotonergic neurotransmission, particularly upregulation of postsynaptic 5-HT1B receptors in the nucleus accumbens and ventral pallidum may be critically involved in the antidepressant action of ketamine. 
Figure 3. DM may promote antidepressant-relevant neural plasticity through sigma-1 and AMPA receptors. Sigma-1 receptor activation facilitates the maturation of pro-BDNF into BDNF and the secretion of BDNF, and increases NGF activity. DM may also increase AMPA receptor function and/or expression through sigma-1 receptors or through other mechanisms and further raise BDNF levels. BDNF and NGF then activate TrkB and TrkA, respectively, which leads to enhancement of downstream signaling pathways to promote neural adaptations and plasticity to ultimately produce antidepressant effects. DM, dextromethorphan; AMPA-R, alpha-amino-3-hydroxy-5-methyl-4-isoxazole propionic acid receptor; BDNF, brain-derived neurotrophic factor; NGF, nerve growth factor; TrkA, tropomyosin receptor kinase A; TrkB, tropomyosin receptor kinase B.

extracellular

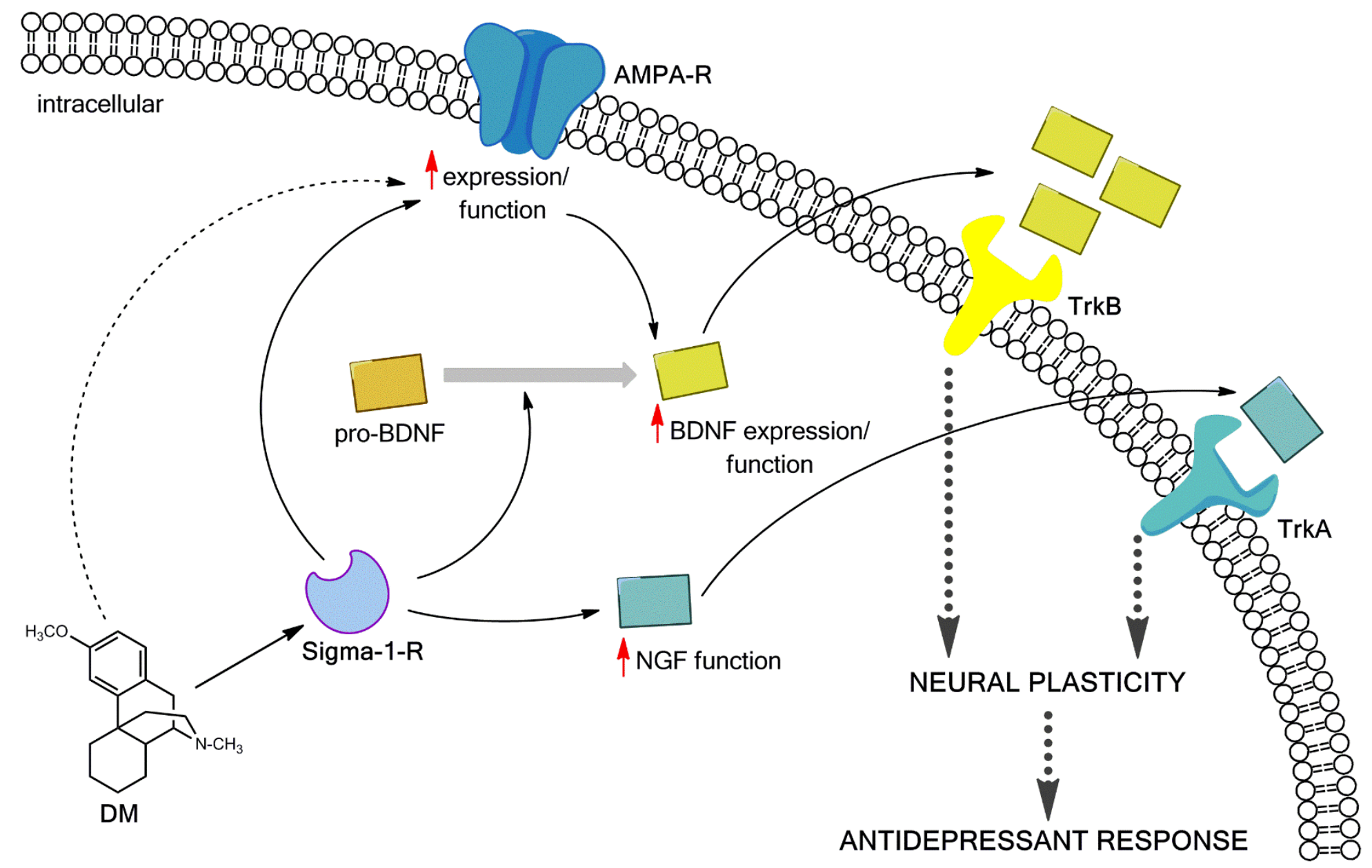


Similarly, a delay in therapeutic response with conventional antidepressants may be due in part to desensitization of alpha-2 noradrenergic autoreceptors (Esteban et al., 1999; Invernizzi and Garattini, 2004). Notably, mirtazapine, a noradrenergic and specific serotonergic antidepressant (NaSSA), has been found to have a faster onset of antidepressant action (as early as within the first week of treatment) compared to SSRIs and serotonin-norepinephrine reuptake inhibitors (SNRIs) (Nagao et al., 2013; Watanabe et al., 2011). Its primary mechanism of action is thought to be through blockade of alpha-2 noradrenergic autoreceptors and heteroreceptors, resulting in enhanced release of NE from noradrenergic terminals, and increased 5-HT release from serotonergic terminals, respectively (Croom et al., 2009). Whether or not DM may activate or inhibit 5-HT1B and alpha-2 noradrenergic receptors located presynaptically or postsynpatically remains to be determined.

DM has been found to act as antagonist at nicotinic (alpha-3-beta-4, alpha-4-beta-2, and alpha-7) receptors (Damaj et al., 2005; Hernandez et al., 2000; Lee et al., 2006). There is evidence suggesting that hypercholinergic neurotransmission, which is associated with depressed mood states, may be mediated through excessive neuronal nicotinic receptor activation and that the therapeutic actions of many antidepressants may be partly mediated through inhibition of these receptors (Shytle et al., 2002). Supporting this, a recent study in pheochromocytoma (PC12) cells suggested that the therapeutic effects

produced by ketamine may be the result of a combination of independent but interrelated pharmacological effects at the alpha-7 nicotinic receptors produced by the parent drug and its metabolites (Paul et al., 2014).

\subsection{Potential impact of metabolism on antidepressant action}

DX has a similar pharmacological profile to DM (Table 2, page 17), and has been found to have antitussive, anticonvulsant and neuroprotective effects in many of the same studies as DM (Shin et al., 2011; Tortella et al., 1989; Werling et al., 2007b). The pharmacology of DM's other major metabolite, 3HM, appears to be non-significant (Shin et al., 2011). What role, if any, 3-HM may play in DM's effects is not fully understood. Given their overlapping and complementary binding profiles, exposure of the 
brain to DM and DX (i.e., not inhibiting the rapid metabolism of DM), may theoretically offer greater potential for antidepressant activity (Lauterbach, 2012). Nevertheless, ample evidence suggests that DM can have effects independent of its metabolites, including in vivo studies using focal CNS administration of DM and in vitro studies wherein the protective effects are not likely related to biotransformation of DM to DX (Werling et al., 2007b).

From a safety and routine clinical use perspective, it may be best to control for the rapid metabolism of DM. This is because DX's action as a more potent PCP-like uncompetitive NMDA receptor antagonist is associated with psychotomimetic disturbances (Dematteis et al., 1998; Miller, 2011; Szekely et al., 1991; Zawertailo et al., 1998), thus limiting its therapeutic utility. In addition, administration of DM alone (without quinidine, for instance) is subjected to significant plasma and brain concentration variability, further making it less than ideal for general clinical use. Several investigations have examined the effects of DM in a myriad of other CNS-related disorders (Werling et al., 2007a). The inconsistencies between the many protective effects observed in preclinical studies and limited efficacy seen in clinical settings may in part be due to the fact that CYP2D6 activity was not assessed or controlled for in these studies. Future clinical trials, including those examining patients with MDD should investigate the relationship between DM levels and significant efficacy or safety endpoints depending on the patient population and genotype/phenotype status.

\subsection{Concerns about abuse liability and long-term toxicity}

The pharmacodynamic effects of DM may lead to abuse liability since OTC DM abuse has increased during the 2000's (Wilson et al., 2011). An analysis of 44,206 DM-related poison control center calls, registered in the National Poison Data System from 2000-2010, found the prevalence increased to a peak of 17.6 calls/million in 2006 and plateaued at 15.7 calls/million in 2010 (p=0.002) (Wilson et al., 2011). This trend has raised concerns since several preclinical animal models have demonstrated adverse effects on cognitive performance. Doses of $40 \mathrm{mg} / \mathrm{kg}$ in rats suppressed the potentiation of the field excitatory postsynaptic potential and the population spike $(\mathrm{p}<0.02)$, which suggest DM may impair 
hippocampal long-term potentiation (LTP) essential for learning (Krug et al., 1993). DM-induced memory impairments in spatial learning were dose-dependent and led to declining performance in the Morris water maze (Bane et al., 1996). These cognitive impairments appeared to become permanent, since water maze learning was also impaired in older rats (18 months) that were only given $40 \mathrm{mg} / \mathrm{kg}$ doses of DM during the adolescent period (28-37 days) (Zhang et al., 2007). Other NMDA antagonists with similar patterns of suppressed LTP, like MK-801, have caused morphological damage in rat cortical neurons that could be prevented by both anticholinergic and benzodiazepine pretreatment (Olney et al., 1991). In humans, DM 100-300 mg/70 kg produced acute and temporary impairments in working memory, episodic memory, attention and metacognition (Carter et al., 2013). At higher dosages, 400 mg/70 kg, DM produced subjective effects (perceptual changes, end-of-session drug liking and mysticaltype experiences) similar to the classic hallucinogen psilocybin according to volunteers with a history of prior hallucinogen use (Reissig et al., 2012). Other signs of neurologic toxicity included nystagmus, slurred speech, light-headedness, and fatigue, which were more commonly reported at higher doses of DM (10 mg/kg/d) and occurred within 1-2 h of administration (Hollander et al., 1994).

CYP2D6 activity appears to be an important factor in the psychoactive effects of high doses of DM. In a pilot study comparing the subjective and psychomotor effects of $3 \mathrm{mg} / \mathrm{kg}$ DM in four EMs and two PMs, the authors found that PMs had greater psychomotor impairment on a manual tracking task and more negative subjective effects (e.g., sedation, dysphoria) while EMs reported greater abuse potential (e.g., higher ratings on the visual analog scales of "good" drug effects and drug "liking") (Zawertailo et al., 1998). In a follow up study, $3 \mathrm{mg} / \mathrm{kg}$ DM following pretreatment with $100 \mathrm{mg}$ quinidine resulted in a ten-fold increase in DM plasma concentration compared to placebo pretreatment (Zawertailo et al., 2010). In combination with varying doses of DM, pretreatment with $100 \mathrm{mg}$ quinidine or placebo produced dose-dependent decrements in performance on a manual tracking task and digit symbol substitution test (Zawertailo et al., 2010). Compared to placebo treatment, negative subjective feelings of "unpleasantness" increased, while positive subjective effects such as euphoria and drug liking decreased with quinidine pretreatment (Zawertailo et al., 2010). These altered psychoactive properties of high-dose 
DM, where negative subjective effects become more pronounced and positive effects become blunted, suggests that DM in combination with CYD2D6 inhibitors may reduce the abuse liability (Miller, 2011).

While many of the above studies tested high doses of DM acutely and reported some detrimental cognitive effects, few studies have addressed the long-term use of therapeutic doses of DM and/or abuse of DM. From the case reports of long-term abuse of DM, chronic effects include recurrent mania from use of 100 to $400 \mathrm{~mL}$, or 300 to $1200 \mathrm{mg}$, of DM daily for up to 8 years (Walker and Yatham, 1993), intermittent euphoria (Fleming, 1986), psychological dependence (Wolfe and Caravati, 1995), and severe cognitive deterioration (Hinsberger et al., 1994). Often, distinctions between acute and chronic overdose cannot be readily made, such as in the case of a 23 -year old man who presented to an emergency room with acute intoxication on top of chronic addiction (36 to 48 ounces of DM a day, or 2160 to $2880 \mathrm{mg}$ of DM for up to 5 years) (Wolfe and Caravati, 1995). Supportive care measures for cases of DM acute toxicity may include benzodiazepines for seizures, aggressive cooling for hyperthermia, and naloxone for respiratory depression or coma (Antoniou and Juurlink, 2014). Regarding prolonged use of therapeutic doses of DM, one of the largest clinical trials ( $=553)$, which dosed $30 \mathrm{mg}$ quinidine with $30 \mathrm{mg} \mathrm{DM}$ twice daily for 1 year, found that the most frequently reported treatment-related adverse drug reactions ( $\geq 5 \%$ ) were nausea, dizziness, headache, somnolence, fatigue, diarrhea and dry mouth (Pattee et al., 2014). These side effects occurred early in the treatment course and were largely mild-moderate and transient (Pattee et al., 2014). Although longer term efficacy and tolerability data for DM/quinidine would be beneficial, the safety and tolerability profile of DM/quinidine in this 1 year study (Pattee et al., 2014) and in earlier studies (which were 12 to 24 weeks in duration) (Yang and Deeks, 2015) suggests that therapeutic doses of DM may not have the same neurologic toxicity reported with higher doses and chronic abusers.

\subsection{Clinical evidence of antidepressant efficacy}

There have been no published reports of DM for the treatment of MDD. However, there has been a positive case report of DM/quinidine in a single case of a depressed patient with emotional lability 
(Messias and Everett, 2012). In the case report, a 32-year-old woman with MDD, recurrent and treatmentrefractory (failed trials with bupropion, escitalopram, paroxetine, citalopram, duloxetine, desvenlafaxine, selegiline patch, ECT, mirtazapine, venlafaxine, and escitalopram as well as received weekly

psychotherapy sessions), had significant problems in controlling her affective expressions, especially her crying outbursts (Messias and Everett, 2012). She was started on off-label treatment with DM/quinidine 20/10 mg daily (Messias and Everett, 2012). At the time of the writing of the case report, the patient has been on this treatment combination for 1 year and reports significant improvement in her mood lability and crying spells, which has allowed her to benefit from her psychotherapy (Messias and Everett, 2012). It is unclear if DM/quinidine helped improve her depression separately or as a consequence of resolving her emotional lability. It is possible that the underlying altered mechanism for affective expression is also altered in mood disorders (Messias and Everett, 2012), and that treatment with DM/quinidine helped improved both the affective and mood disorder.

$\mathrm{DM}$ (30 mg or $60 \mathrm{mg} /$ day) has also been examined in randomized, placebo-controlled trials as an add-on to VPA for bipolar disorder for 12 weeks (Chen et al., 2014; Lee et al., 2012). In both studies, treatment with and without DM significantly improved scores in the Young Mania Rating Scale (YMRS) and HDRS; the differences between groups were non-significant (Chen et al., 2014; Lee et al., 2012). Noteworthy, in the 2014 study, which also measured plasma BDNF levels in the patients, changes in plasma BDNF levels were significantly greater in the DM $(60 \mathrm{mg} / \mathrm{kg})$ group than in the placebo group (Chen et al., 2014), suggesting the addition of DM may provide more neurotrophic effects than VPA alone. It would be interesting to see whether addition of quinidine to slow down the rapid metabolism of DM would potentiate the neurotrophic effects and result in greater therapeutic effects.

Finally, and most importantly, in depressed patients with treatment resistant bipolar type II or bipolar not otherwise specified disorder, some had reported improvements in mood within 1-2 days after initiating treatment with DM/quinidine or having the dose increased to twice a day (Kelly and Lieberman, 2014). This study was a retrospective chart review of 77 patients who all had been experiencing depressive symptoms for at least two years, and the mean number of failed medication trials was 21.2 
(Kelly and Lieberman, 2014). DM/quinidine 20/10 mg once daily, or twice daily if no improvements were seen in the first three weeks, was added to the patients' drug regimen, with no changes to the pre-existing drug regimen during the course of treatment with DM/quinidine (Kelly and Lieberman, 2014). After 90 days, the average Clinical Global Impression-Improvement (CGI-I) score, which was the primary outcome measure in the study, was +1.66 ( 1 =slightly improved, $2=$ much improved) $(\mathrm{N}=59)$ (Kelly and Lieberman, 2014). Though the retrospective nature of and lack of a control group in this study come with many limitations and conclusions about causation cannot be made, the long duration of the continuous or near continuous depressive symptoms for at least 2 years prior to starting DM/quinidine makes extemporaneous improvement that lasted 90 days or more unlikely (Lee et al., 2012). In addition, three patients who experienced a favorable response to DM/quinidine (+2 CGI-I) had discontinued it for nonclinical reasons (Kelly and Lieberman, 2014). After discontinuing DM/quinidine, all 3 experienced worsening symptoms of depression (Kelly and Lieberman, 2014). When DM/quinidine was restarted, they all regained their prior level of improvement (Kelly and Lieberman, 2014). This naturalistic off-onoff-on improvement experienced by 3 patients also supports a cause and effect relationship (Kelly and Lieberman, 2014). In addition, while no formal inquiry was made for the patients in the present study, the authors noted that a number of patients of their own volition had remarked how rapidly DM/quinidine worked, often within 1-2 days, of starting DM/quinidine or when increasing it to twice a day dosing (Kelly and Lieberman, 2014). These promising findings suggest that DM not only has antidepressant efficacy in TRD, but also rapid acting antidepressant effects. 


\section{CHAPTER 3}

Involvement of sigma-1 receptors in the antidepressant-like effects of DM 


\subsection{Introduction}

In the studies herein, we tested the hypothesis that DM can exert antidepressant-like actions at least in part through sigma-1 receptors. First, the ability of DM to cause antidepressant-like effects was examined in the forced swim test (FST). The FST is the most validated behavioral assay for predicting antidepressant efficacy (Cryan and Holmes, 2005; McArthur and Borsini, 2006; Nestler et al., 2002b) and thus provides a rational format for the initial evaluation of the antidepressant potential of DM. In addition, the effect of DM on locomotor activity in the open field test (OFT) was measured to determine whether stimulant effects could account for its apparent antidepressant-like actions. The conventional antidepressant drugs imipramine and fluoxetine and fast acting antidepressant drug ketamine were used as reference ligands for these behavioral tests. Second, to evaluate the potential involvement of sigma-1 receptors in the in vivo antidepressant-like actions of DM, pharmacological antagonists targeting sigma-1 receptors were examined for their ability to prevent the antidepressant-like effects of DM. Third, since DM undergoes extensive first-pass metabolism by CYP2D6 to its major active metabolite DX (Schmid et al., 1985), the CYP2D6 inhibitor quinidine was administered concomitantly with DM to raise the plasma concentration and bioavailability of DM (Pope et al., 2004) and determine whether the metabolism of DM affects its antidepressant efficacy. Finally, to define the manner in which DM binds to sigma-1 receptors (competitive and/or non-competitive), saturation binding studies were conducted.

\subsection{Results}

3.2.1. Conventional and fast acting antidepressants dose response

The TCA imipramine served as a positive control and significantly altered immobility time in the FST (Figure 4A; F[2,32]=10.24, P<0.05). Post-hoc Dunnett's test showed that imipramine at $20 \mathrm{mg} / \mathrm{kg}$ significantly decreased immobility time when compared to saline $(\mathrm{q}=4.52, \mathrm{P}<0.001)$. Imipramine did not alter locomotor activity, even at doses that produced antidepressant-like effects (Figure 4B; F[2,30]=0.23, n.s.). Consistent with earlier reports that the FST does not reliably detect the effects of SSRIs (Cryan et al., 2002), the SSRI fluoxetine did not significantly reduce immobility time in the FST under the 
Figure 4. Antidepressant-like effects of imipramine, ketamine, and DM in the FST. Imipramine significantly decreased immobility time (A), but had no significant effects on locomotor activity (B). Ketamine significantly decreased immobility time (C), and increased locomotor activity (D). DM significantly decreased immobility time (E), and increased locomotor activity (F). There was no correlation between DM-induced locomotor alterations and decreased immobility times $(\mathrm{G})$. Data shown are expressed as mean $\pm \mathrm{SEM}$. $* \mathrm{P}<0.05$, $* * \mathrm{P}<0.01$, $* * * \mathrm{P}<0.001$, compared with the saline-treated group; one-way ANOVA followed by post-hoc Dunnett's tests. Pearson's r correlation test for correlation analysis.

A.

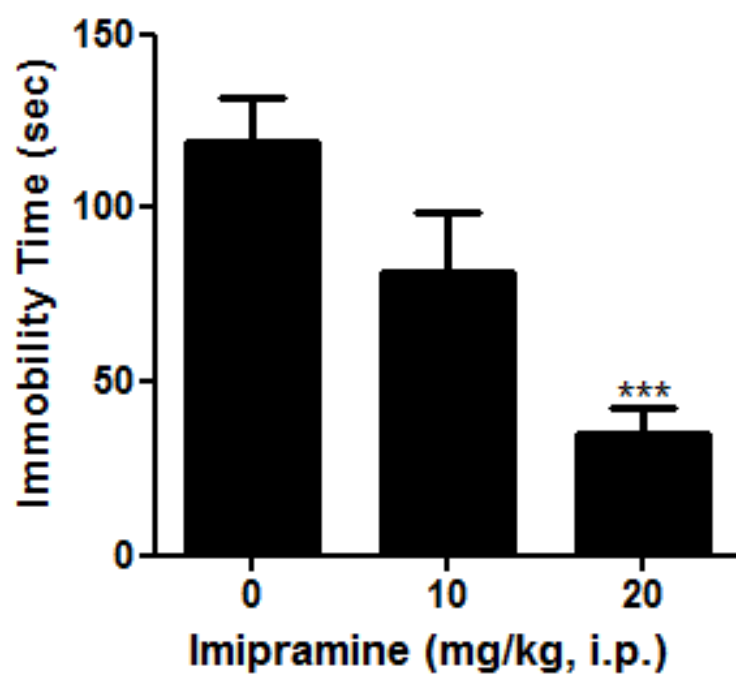

C.

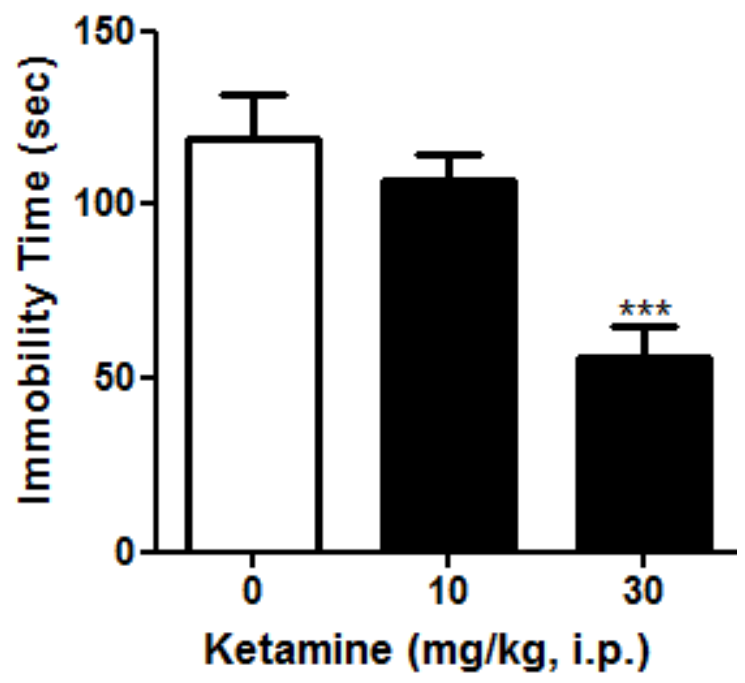

B.

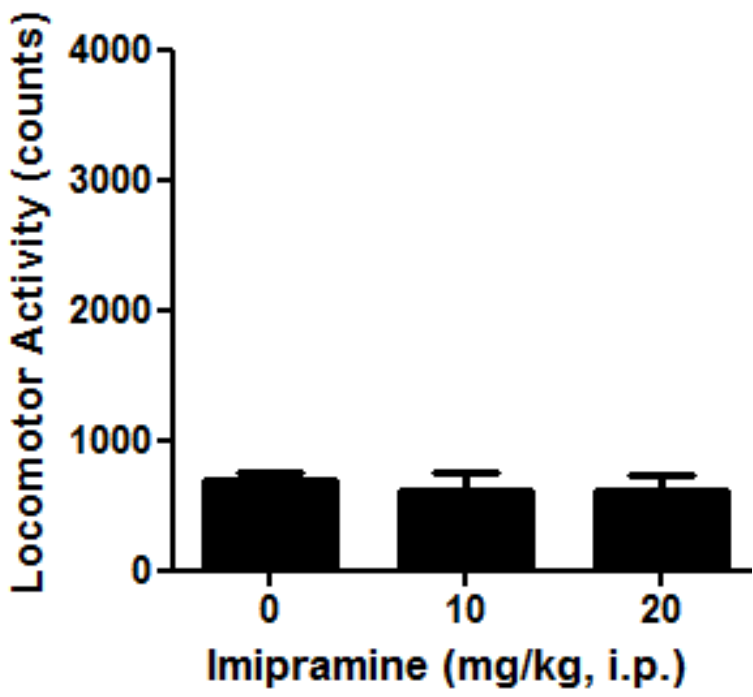

D.

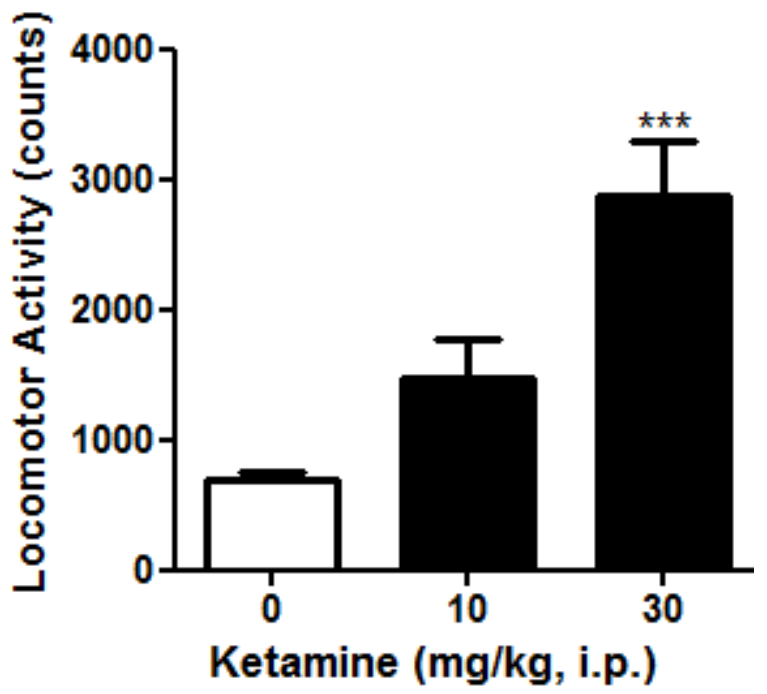



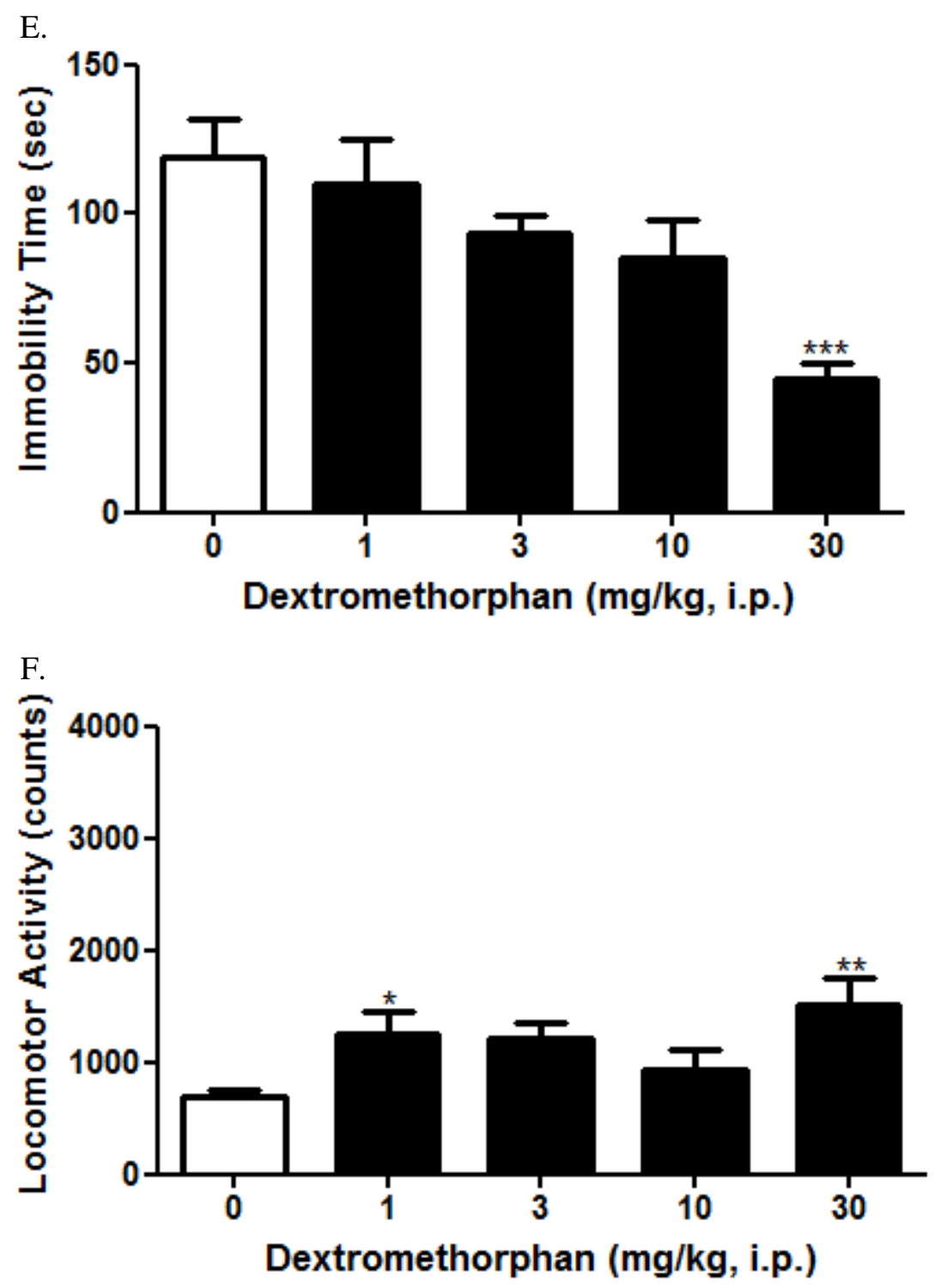

G.

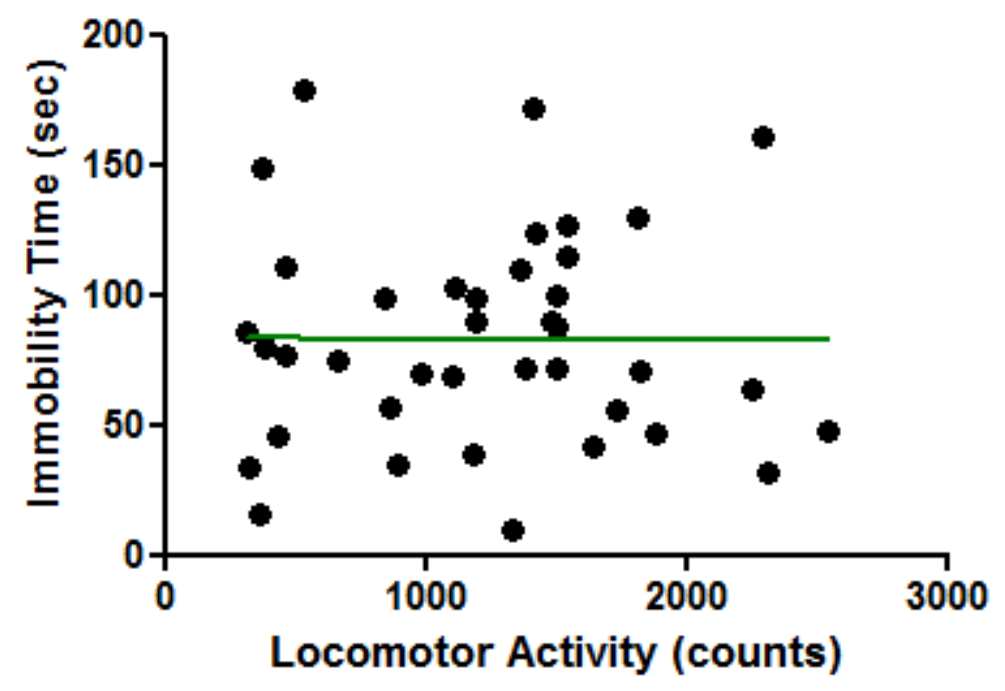


conditions used by our laboratory $(\mathrm{F}[2,32]=1.97$, n.s.); it also did not alter locomotor activity $(F[2,30]=2.36$, n.s. $)$.

The fast acting antidepressant ketamine significantly altered immobility time (Figure 4C; $\mathrm{F}[2,34]=9.59, \mathrm{P}<0.001)$. Post-hoc Dunnett's test showed that ketamine at $30 \mathrm{mg} / \mathrm{kg}$ significantly decreased immobility time when compared to saline $(\mathrm{q}=5.12, \mathrm{P}<0.001)$. In the OFT, however, ketamine also significantly altered locomotor activity (Figure 4D; F[2,33]=13.28, P<0.0001), with post-hoc Dunnett's test showing that ketamine at $30 \mathrm{mg} / \mathrm{kg}$ significantly increased locomotor activity $(\mathrm{q}=5.12$, $\mathrm{P}<0.001)$.

\subsubsection{DM dose response}

DM significantly reduced immobility time (Figure 4E; F[4,50]=6.16, $\mathrm{P}<0.001$ ), with a post-hoc Dunnett's test revealing that $30 \mathrm{mg} / \mathrm{kg}$ differed significantly from saline $(\mathrm{q}=4.70, \mathrm{P}<0.05)$. DM also produced significant effects on locomotor activity (Figure 4F; F[4,48]=4.27, P<0.05), with post-hoc Dunnett's tests confirming that the following doses of DM elicited effects that differed significantly from the saline control: $1 \mathrm{mg} / \mathrm{kg}(\mathrm{q}=2.63, \mathrm{P}<0.05)$ and $30 \mathrm{mg} / \mathrm{kg}(\mathrm{q}=3.81, \mathrm{P}<0.01)$. A correlation analysis between locomotor activity and immobility time was carried out to determine whether stimulant effects could account for its apparent antidepressant-like actions. The Pearson's $r$ correlation test revealed that there was no correlation between the DM-induced increase in locomotor activity and decrease in immobility time (Figure 4G; r=-0.01, n.s.)

\subsubsection{Sigma-1 receptor antagonists dose response}

When tested alone, the sigma-1 receptor antagonist BD1063 (1-[2-(3,4-Dichlorophenyl)ethyl]-4methylpiperazine $)$ displayed a significant effect on immobility time $(\mathrm{F}[3,36]=4.11, \mathrm{P}<0.05)$. Post-hoc Dunnett's tests showed that BD1063 significantly reduced immobility time at $30 \mathrm{mg} / \mathrm{kg}(\mathrm{q}=3.41, \mathrm{P}<0.01)$, but not at $10 \mathrm{mg} / \mathrm{kg}$ (q=1.82, n.s.). Consequently, subsequent antagonist testing in the FST was performed with the $10 \mathrm{mg} / \mathrm{kg}$ dose of BD1063 which did not have effects on its own. Alone, BD1063 also produced 
significant changes in locomotor activity $(\mathrm{F}[3,34]=5.81, \mathrm{P}<0.05)$. Post-hoc Dunnett's tests revealed that only the low $3 \mathrm{mg} / \mathrm{kg}$ dose caused a significant increase in activity for BD1063 (q=2.73, P<0.05), thereby indicating that the alterations in locomotor behavior did not account for the changes observed in the FST.

The second sigma-1 receptor antagonist BD1047 (N-[2-(3,4-Dichlorophenyl)ethyl]-N-methyl-2(dimethylamino)ethylamine) alone displayed no significant effects in the FST (F[3,36]=2.55, n.s.). In the OFT, BD1047 produced an effect that significantly differed from saline injections $(F[3,34]=6.99$, $\mathrm{P}<0.001)$. Post-hoc Dunnett's tests revealed that only the low $3 \mathrm{mg} / \mathrm{kg}$ dose caused a significant increase in activity for BD1047 (q=3.70, $\mathrm{P}<0.01)$.

\subsubsection{Effects of sigma-1 receptor antagonists in the presence of DM, imipramine, or ketamine}

Pretreatment of mice with behaviorally inactive doses of BD1063 or BD1047, two well-

established and selective sigma-1 receptor antagonists, attenuated the antidepressant-like effects of DM, but not imipramine or ketamine (Figure 5). Pretreatment with BD1063 (10 mg/kg) attenuated the antidepressant-like effects of DM (30 mg/kg) (Figure 5A). ANOVA confirmed a significant difference between the various treatment groups in the antagonism study for DM in the FST $(\mathrm{F}[3,41]=5.59, \mathrm{P}<0.01)$. Post-hoc Tukey's multiple comparison tests revealed that the DM alone treatment group differed significantly from saline $(\mathrm{q}=5.60, \mathrm{P}<0.001)$, as well as the BD1063 + DM group $(\mathrm{q}=4.21, \mathrm{P}<0.05)$. Pretreatment of mice with BD1063 also significantly decreased the locomotor stimulatory effect of DM $(30 \mathrm{mg} / \mathrm{kg})$, with an overall significant difference between treatment groups $(\mathrm{F}[3,39]=10.03, \mathrm{P}<0.0001)$. Moreover, post-hoc Tukey's test confirmed that BD1063 treatment blocked the ability of DM to increase locomotor activity $(\mathrm{q}=6.62, \mathrm{P}<0.001)$.

Pretreatment with the second sigma-1 preferring antagonist BD1047 (10 or $20 \mathrm{mg} / \mathrm{kg}$ ) showed a trend toward the attenuation of the antidepressant-like effects of DM (30 mg/kg), with the results of 10 $\mathrm{mg} / \mathrm{kg}$ pretreatment with BD1047 illustrated in Figure 5B. The overall ANOVA was significant for the BD1047 pretreatment study $(\mathrm{F}[5,64]=5.65, \mathrm{P}<0.001)$. Pairwise comparisons using post-hoc Tukey's multiple comparison tests further confirmed that the BD1047 (10 or $20 \mathrm{mg} / \mathrm{kg})+$ DM groups did not 
differ significantly from saline ( $q=4.04$ and 2.77 , n.s.). However, the differences in the effects of DM in the absence and presence of BD1047 (10 or $20 \mathrm{mg} / \mathrm{kg}$ ) were not significant ( $\mathrm{q}=2.52$ and 3.07, n.s.), reflecting partial attenuation of the effects. In the OFT, BD1047 significantly decreased the effects of DM $(\mathrm{F}[5,61]=6.59, \mathrm{P}<0.001)$, with post-hoc Tukey's tests confirming the ability of BD1047 $(10 \mathrm{mg} / \mathrm{kg})$ to block the stimulant effect of DM $(30 \mathrm{mg} / \mathrm{kg})(\mathrm{q}=5.41, \mathrm{P}<0.05)$.

In contrast to the attenuation of DM-induced effects in the FST, pretreatment with BD1063 did not prevent, although it non-significantly reduced, the antidepressant-like effects of imipramine (20 $\mathrm{mg} / \mathrm{kg}$ ) (Figure 5C). Post-hoc Tukey's test confirmed that imipramine significantly reduced immobility time compared to saline $(\mathrm{q}=6.74, \mathrm{P}<0.001)$. Post-hoc comparisons of the BD1063 + Imipramine group showed that its effects did not differ significantly from imipramine alone ( $q=2.00$, n.s.), and there was a significant reduction in immobility time compared to saline $(q=4.55, \mathrm{P}<0.05)$. Locomotor activity differed among the treatment groups $(\mathrm{F}[3,39]=5.49, \mathrm{P}<0.01)$. Although imipramine and BD1063 at the doses tested did not differ from saline ( $\mathrm{q}=1.01$ and 0.13 , respectively, n.s.), the combination of BD1063 + Imipramine produced a significant decrease in locomotor activity $(\mathrm{q}=5.17, \mathrm{P}<0.01)$.

Likewise, pretreatment with BD1063 did not significantly attenuate the antidepressant-like effects of ketamine $(30 \mathrm{mg} / \mathrm{kg})$ (Figure 5D). The overall ANOVA was significant $(\mathrm{F}[3,49]=5.45, \mathrm{P}<0.01)$, and post-hoc Tukey's test confirmed that ketamine significantly differed from saline $(\mathrm{q}=5.62, \mathrm{P}<0.01)$. However, the BD1063 + Ketamine group did not significantly differ from ketamine alone (q=1.61, n.s.) and also from saline ( $\mathrm{q}=3.41$, n.s.), reflecting partial attenuation. In the OFT, locomotor activity differed among the treatment groups $(\mathrm{F}[3,47]=10.74, \mathrm{P}<0.0001)$. Both the ketamine alone and BD1063+ Ketamine groups produced a significant increase in locomotor activity $(\mathrm{q}=7.05, \mathrm{P}<0.001$; and $1=3.82$, $\mathrm{P}<0.05$, respectively).

\subsubsection{Effects of sigma-1 antagonist BD1063 on DM dose response}

When testing a single dose of BD1063 (10 mg/kg) against different doses of DM, the presence of BD1063 elicited a shift to the right in the DM dose response curve (Figure 6A). However, the animals 
Figure 5. Blockade of sigma-1 receptors prevented the antidepressant-like effects of DM, but not imipramine and ketamine in the FST. Pretreatment with the sigma-1 receptor antagonist BD1063 prevented the DM-induced decrease in immobility time (A). BD1047 pretreatment also produced a noticeable, albeit not statistically significant, trend toward the prevention of the decreased immobility time induced by DM (B). In contrast, the antidepressant-like effect of imipramine and ketamine were not significantly prevented by BD1063 pretreatment (C and D,

respectively). Data shown are expressed as mean \pm SEM. $* \mathrm{P}<0.05$, $* * * \mathrm{P}<0.001$, compared with the saline-treated group; \#P<0.05, compared with the DM-treated group; one-way ANOVA followed by post-hoc Tukey's tests. DM, dextromethorphan; IM, imipramine; KET, ketamine.

A.

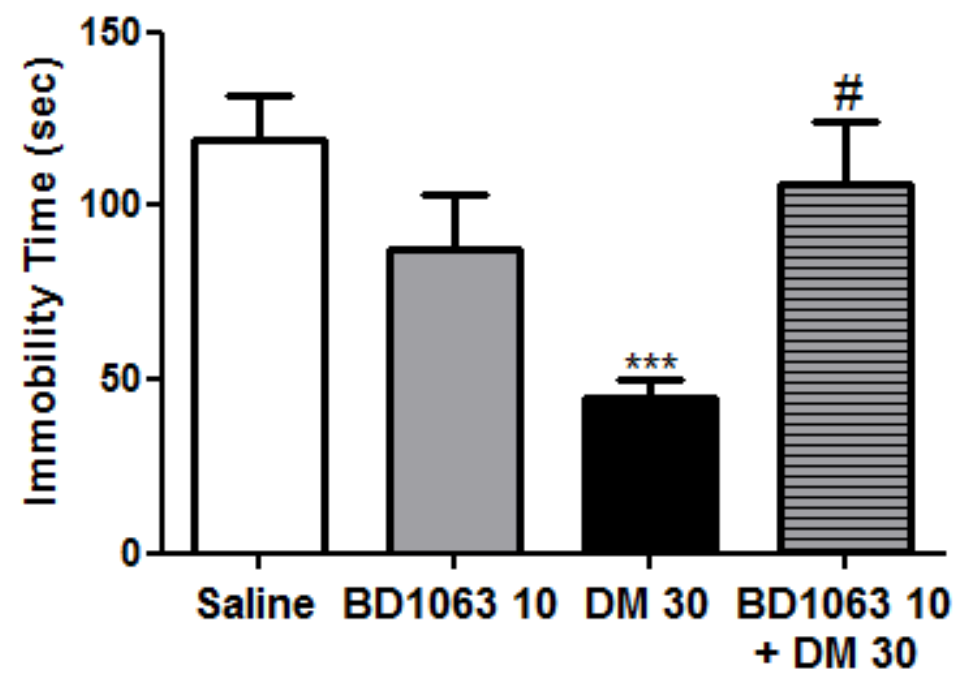

$B$.

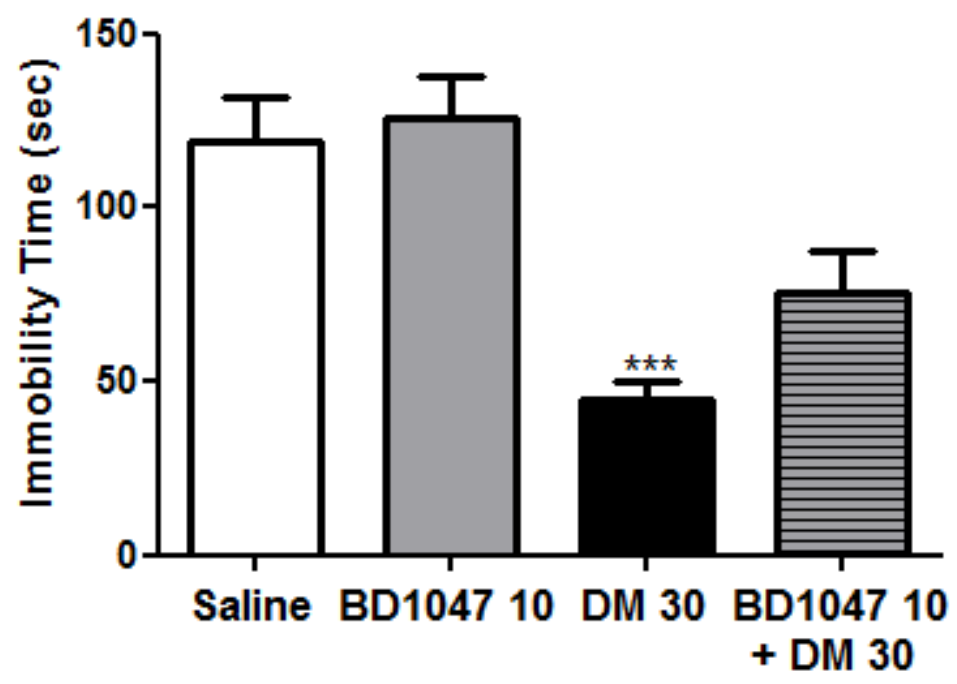



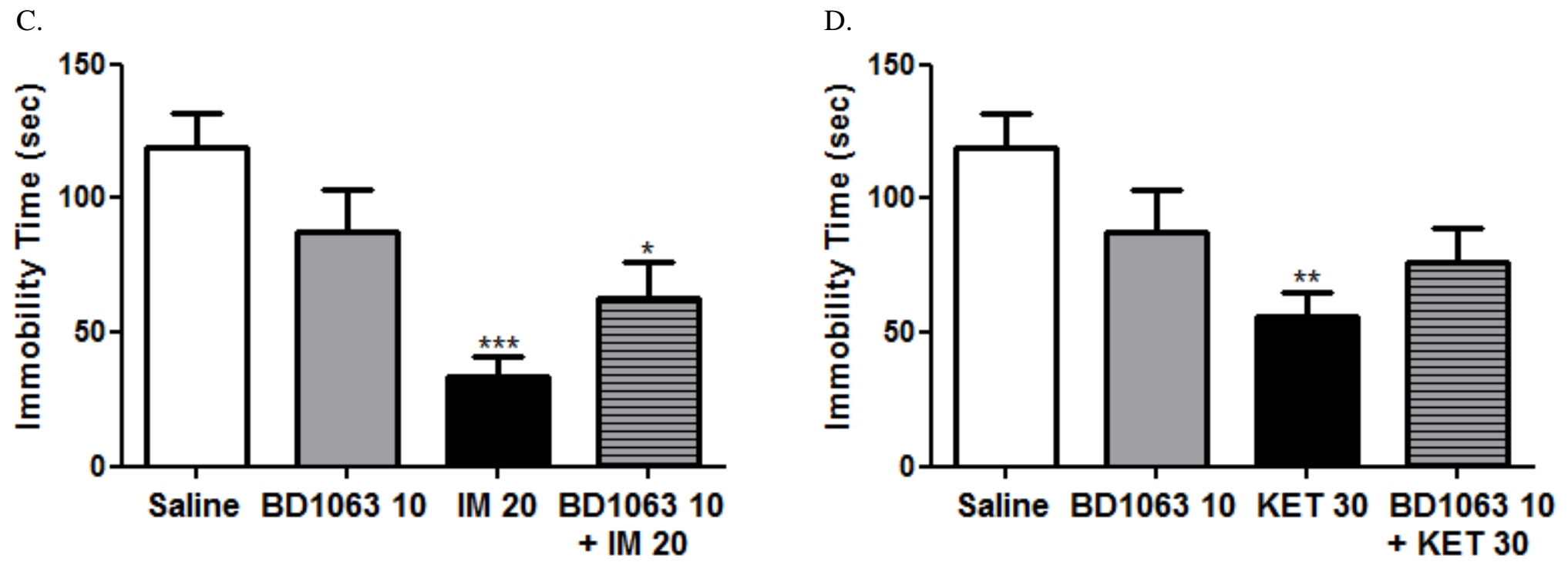
Figure 6. Competitive antagonism of the behavioral effects of DM by BD1063 in the FST. A single dose of BD1063 (10 mg/kg) pretreatment shifted the DM dose response curve to the right in the FST (A), and blocked the DM-induced stimulatory effect in the OFT (B). Data shown are expressed as mean \pm SEM. *P $<0.05$, ** $\mathrm{P}<0.01$ compared with the DM $(30 \mathrm{mg} / \mathrm{kg})$-treated group; one-way ANOVA followed by post-hoc Tukey's tests. Solid black line, without BD1063. Dashed gray line, with BD1063.

A.

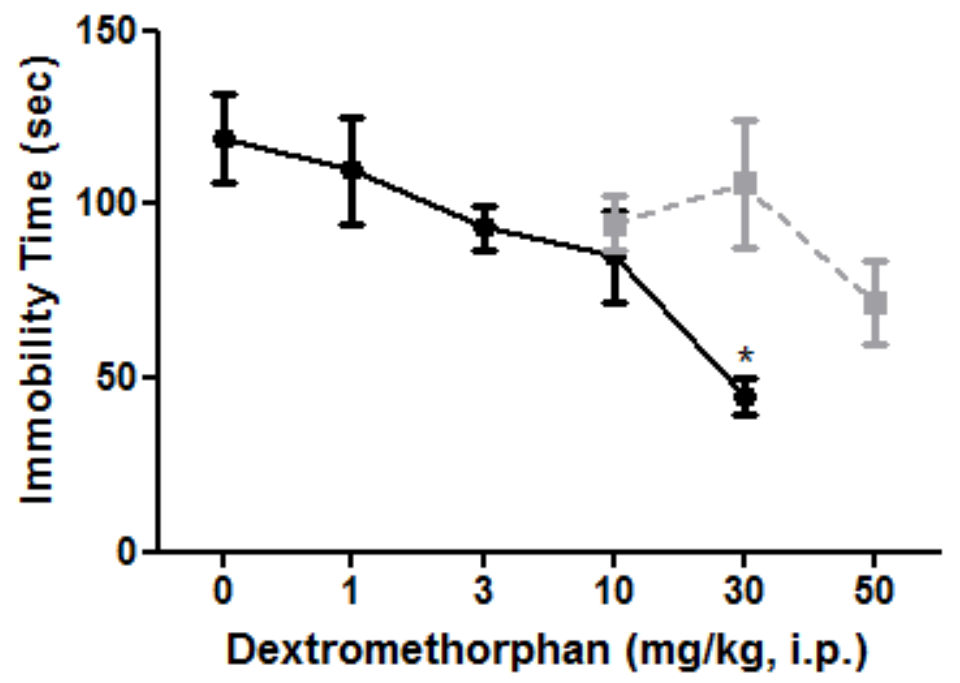

B.

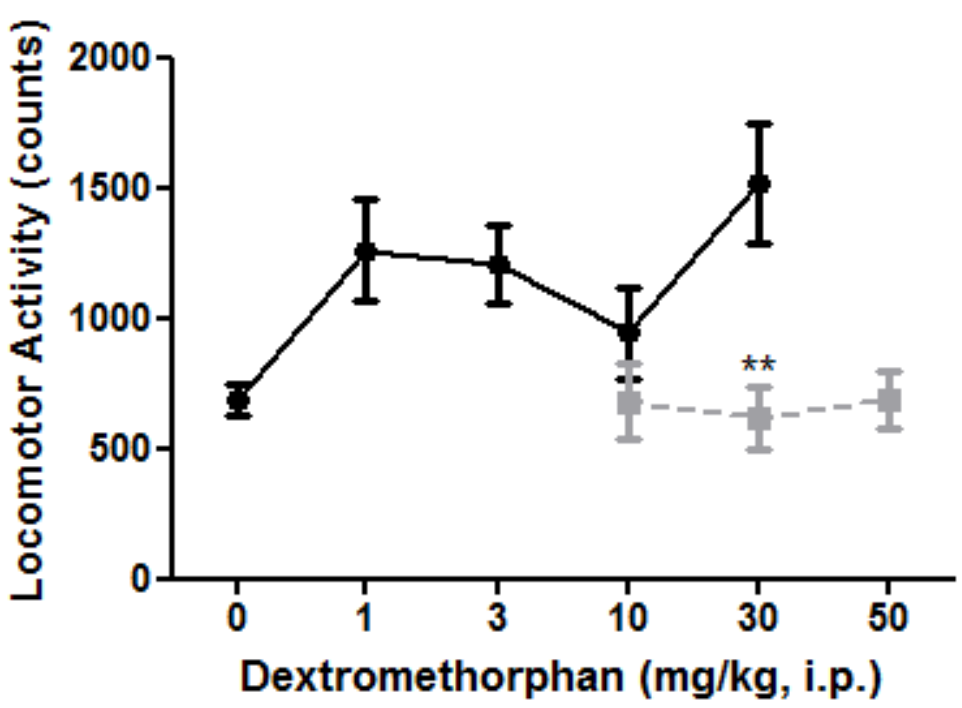


exhibited abnormal behaviors, particularly vocalization, during the locomotor tests beginning at the 50 $\mathrm{mg} / \mathrm{kg}$ dose of DM in combination with BD1063. Higher doses (up to $100 \mathrm{mg} / \mathrm{kg}$ ) of DM were lethal, which limited the extent to which the dose response could be characterized. In the OFT, when testing the single dose of BD1063 against increasing doses of DM, BD1063 appeared to block the DM-induced locomotor activity (Figure 6B). However, as mentioned above, the testing of higher doses of DM was not carried out due to behavioral toxicity.

\subsubsection{Effects of CYP2D6 inhibitor quinidine on DM dose response}

Concurrent administration of quinidine $(30 \mathrm{mg} / \mathrm{kg})$, which inhibits the CYP2D6 metabolism of DM (Pope et al., 2004), potentiated the antidepressant-like effect of DM (10 mg/kg) (Figure 7A). ANOVA confirmed a significant difference between the various treatment groups $(F[7,77]=8.45$, $\mathrm{P}<0.0001)$. Post-hoc Tukey's multiple comparison tests revealed that the DM $(30 \mathrm{mg} / \mathrm{kg})$ treatment group differed significantly from saline alone $(\mathrm{q}=6.96, \mathrm{P}<0.001)$ and in combination with quinidine $(\mathrm{q}=7.11$, $\mathrm{P}<0.001)$. Importantly, while $\mathrm{DM}$ at $10 \mathrm{mg} / \mathrm{kg}$ alone was not significantly different from saline $(\mathrm{q}=3.19$, n.s.), it produced a significant decrease in immobility time when combined with quinidine (q=6.78, $\mathrm{P}<0.001$ ). In the OFT, DM in combination with quinidine had no stimulant effects (Figure 7B). The overall ANOVA was significant for the various treatment groups $(\mathrm{F}[7,73]=3.97, \mathrm{P}<0.001)$. Pairwise comparisons using post-hoc Tukey's multiple comparison tests further confirmed that the DM (30 mg/kg) alone treatment group differed significantly from saline $(\mathrm{q}=6.40, \mathrm{P}<0.001)$. In combination with quinidine, however, DM had no significant stimulant effects $(\mathrm{q}=4.04$, n.s. $)$. When tested alone, quinidine had no significant effects in the FST ( $t=0.47$, n.s.) nor did it have any effect on locomotor activity in the OFT (t=0.99, n.s.).

\subsubsection{DM binding to sigma-1 receptors}

The results of the sigma- 1 receptor saturation binding assays demonstrated there was a significant reduction in $\mathrm{K}_{\mathrm{d}}$ and $\mathrm{B}_{\max }$ when the assays were performed in the presence of DM, compared to when no 
DM was added to the assay (Table 3). Unpaired t-tests revealed a significant decrease in both $\mathrm{K}_{\mathrm{d}}(\mathrm{t}=3.87$, $\mathrm{P}<0.05)$ and $\mathrm{B}_{\max }(\mathrm{t}=4.29, \mathrm{P}<0.05)$ with the inclusion of $400 \mathrm{nM} \mathrm{DM}$ in the assays.

\subsection{Discussion}

This study is the first to show that DM has antidepressant-like effects in vivo, in addition to implicating sigma-1 receptors as a mechanism contributing to its antidepressant actions. Moreover, concomitant administration of the CYP2D6 reversible inhibitor quinidine potentiated the effects of DM in the FST. This demonstrates that the antidepressant-like effects of DM do not require a significant degree of conversion to the metabolite DX, and suggests DM itself has antidepressant efficacy.

The antidepressant-like effects of DM appear to involve sigma-1 receptors. In the current study, two well-established sigma-1 receptor antagonists (BD1063 and BD1047) reduced the antidepressant-like actions of DM in vivo. They are thought to act in a competitive manner since in the presence of BD1063, the dose response curve for DM was shifted to the right. An involvement of sigma- 1 receptors in the antidepressant-like effects of DM is consistent with earlier reports that selective sigma-1 receptor agonists can on their own reduce immobility time in the FST (Matsuno et al., 1996; Skuza and Rogoz, 2002; Urani et al., 2001; Wang et al., 2007) and produce antidepressant-like effects in other animal models such as the tail suspension test (TST) and olfactory bulbectomy (Bermack et al., 2002; Ukai et al., 1998). Thus, additional studies involving these and other animal models used in depression research (e.g., sucrose preference test, novelty suppression) (Cryan and Holmes, 2005; McArthur and Borsini, 2006; Nestler et al., 2002b) will be needed in the future to further evaluate the antidepressant potential of DM and the involvement of sigma-1 receptors.

The ability of DM to elicit antidepressant-like actions through sigma-1 receptors suggests future studies to evaluate potential fast acting therapeutic effects are also warranted. Sigma-1 receptor agonists can enhance serotonergic neuronal firing in the rat dorsal raphe nucleus after only 2 days vs. 2 weeks of treatment that is typically required of conventional antidepressant drugs (Bermack and Debonnel, 2001; Lucas et al., 2008). In addition, the fast acting antidepressant drug ketamine has recently been shown to 
Figure 7. Potentiation of the antidepressant-like effects of DM by quinidine in the FST. A single dose of the CYP2D6 inhibitor quinidine $(30 \mathrm{mg} / \mathrm{kg}$ ) administered concomitantly with DM significantly potentiated the decrease in immobility time for DM at $10 \mathrm{mg} / \mathrm{kg}$ (A). In contrast, in the OFT, DM in combination with quinidine had no stimulatory effects (B). Data shown are expressed as mean $\pm \mathrm{SEM}$. $* * * \mathrm{P}<0.001$, compared with the saline-treated group; one-way ANOVA followed by post-hoc Tukey's tests. Black bar (ם) with quinidine. White bar ( $\square$ ) without quinidine.
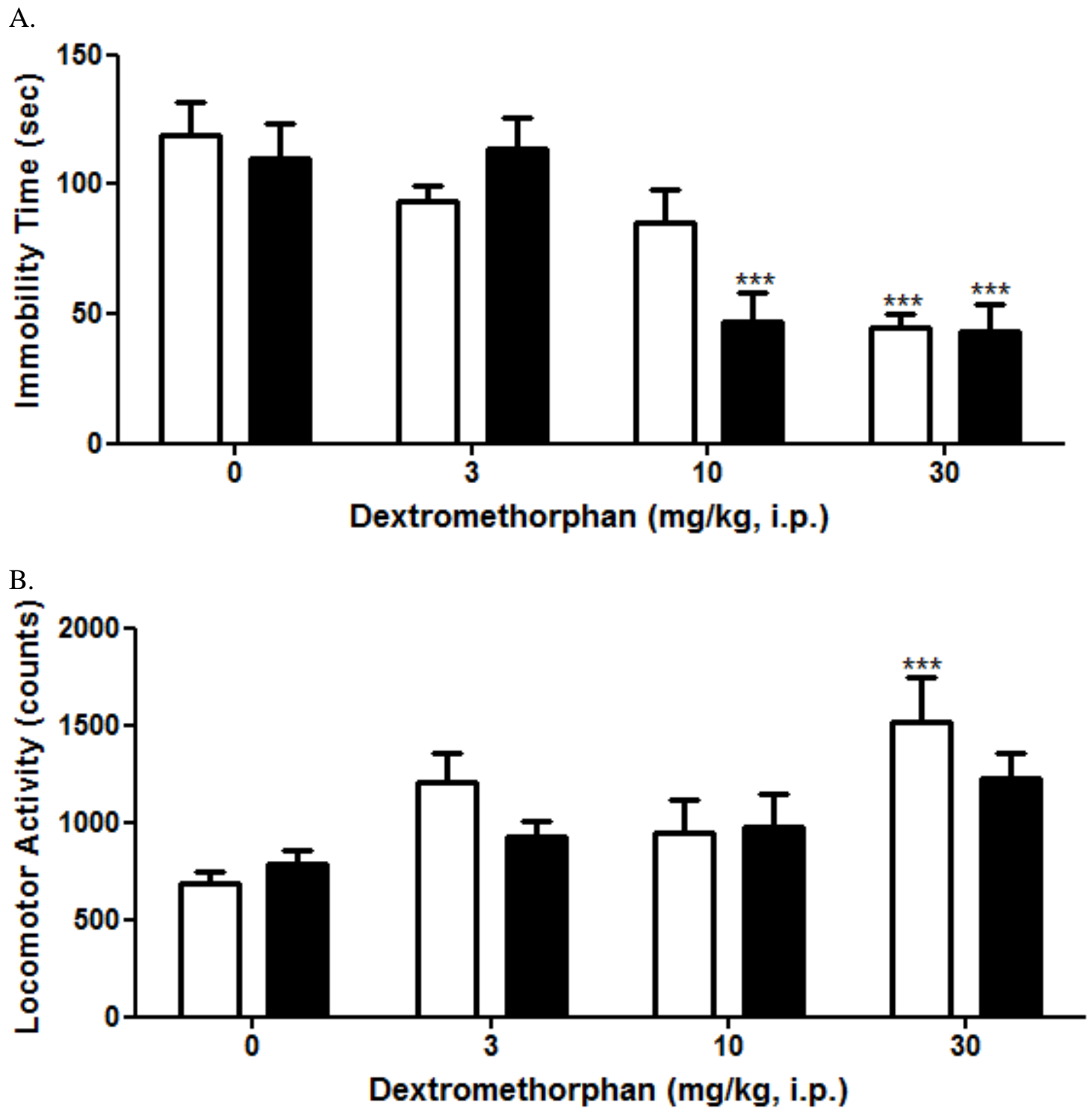

Table 3. Binding parameters for sigma-1 receptors in the absence and presence of DM. Saturation binding assays in brain homogenates for sigma- 1 receptors were conducted using $\left[{ }^{3} \mathrm{H}\right](+)$-pentazocine as the radioligand. The assays were performed in the absence or presence of DM (400 nM). The $K_{d}$ and $B_{\max }$ were determined using nonlinear regression. DM produced a significant decrease in $K_{d}$ and $B_{\max }$. Data shown are expressed as mean \pm SEM. ${ }^{a} \mathrm{P}<0.05$, compared with $\left[{ }^{3} \mathrm{H}\right](+)$-pentazocine alone; unpaired t-test.

\begin{tabular}{|l|l|l|}
\hline Assay condition & $\mathbf{K}_{\mathbf{d}}(\mathbf{n M})$ & $\mathbf{B}_{\max }(\mathbf{f m o l} / \mathbf{m g}$ protein $)$ \\
\hline No additional compound & $27.38 \pm 2.23$ & $356 \pm 12$ \\
\hline+ DM 400 nM & $16.81 \pm 1.58^{\mathrm{a}}$ & $290 \pm 9^{\mathrm{a}}$ \\
\hline
\end{tabular}


potentiate NGF-induced neurite outgrowth through a sigma-1-dependent mechanism (Robson et al., 2012), supporting the emerging importance of sigma-1 receptors in modulating neuronal plasticity, which itself is a critical element for conveying both rapid and delayed antidepressant activity.

Earlier competition binding studies showed that DM has significant affinity for sigma-1 receptors (138-652 nM) (Chou et al., 1999; Fishback et al., 2012; Nam et al., 2012; Werling et al., 2007b), and thus further characterization of the interaction of DM with sigma-1 receptors was undertaken in the current study. The saturation binding studies indicate that the interaction of DM with sigma-1 receptors is complex, with both a change in $B_{\max }$ and $\mathrm{K}_{d}$ in the binding of $\left[{ }^{3} \mathrm{H}\right](+)$-pentazocine in the presence of DM. The reduction in the number of sigma- 1 receptors $\left(B_{\max }\right)$ with which $\left[{ }^{3} \mathrm{H}\right](+)$-pentazocine binds suggests non-competitive interactions of DM with sigma-1 receptors. However, there is also a decrease in $\mathrm{K}_{\mathrm{d}}$ for $\left[{ }^{3} \mathrm{H}\right](+)$-pentazocine binding in the presence of DM, suggesting additional competitive interactions. Together, the data support the presence of at least two distinct sites or modes of interaction with which DM binds to the sigma-1 receptor, one with which it has competitive interactions, and another with which it has non-competitive interactions. This interpretation would be consistent with other reports of multiple regions for ligand interactions on the sigma-1 receptor, some of which have functional ramifications for agonist vs. antagonist activity (Cobos et al., 2005; Wu and Bowen, 2008; Yamamoto et al., 1999). The affinity differences of DM for its two putative binding sites appear to be similar ( $<100$-fold difference) since competition binding assays of DM at sigma-1 receptors are consistent with a one-site fit (Fishback et al., 2012). The antidepressant-like effects of DM are thought to be mediated through the competitive binding site since i) there appears to be a rightward shift in its dose response curve in the FST with no apparent change in maximal effect, and ii) (+)-pentazocine, the sigma-1 receptor agonist used to label the receptor, has previously also been reported to produce similar antidepressant-like effects (Fishback et al., 2010; Matsuno et al., 1996).

In addition to interacting with sigma-1 receptors, DM has been reported to alter monoamine reuptake, particularly 5-HT and NE at $\mathrm{K}_{\mathrm{i}}$ values of 23 and $240 \mathrm{nM}$, respectively (Codd et al., 1995), which have implications for antidepressant effects in humans. The significant affinity of DM for SERT 
(40 nM) (Werling et al., 2007a) would be expected to contribute to antidepressant efficacy in humans, although it would not account for potential fast acting effects, nor reductions in immobility time herein. Under the experimental parameters used in the current study, the classical SSRI fluoxetine did not produce significant reductions in immobility time in the FST. This is consistent with the reports of others that the FST does not reliably detect the antidepressant potential of SSRIs (Cryan et al., 2002). Thus, this mechanism, which is a known contributor to antidepressant efficacy in humans, is unlikely to account for the pattern of antidepressant-like effects observed with DM herein. In contrast to its high affinity for SERT, DM binds much more weakly with NET (> $1 \mu \mathrm{M})$ (Werling et al., 2007a), but its reported ability to modulate NE reuptake (Codd et al., 1995) would be expected to contribute conventional antidepressant effects under clinical conditions.

Compared to the ability of BD1063 pretreatment to significantly block the antidepressant-like effects of DM, it failed to attenuate that of imipramine, which has an overlapping binding profile with DM: SERT (1.3-20 nM) (Bymaster et al., 2002; Owens et al., 1997; Runyon et al., 2001; Tatsumi et al., 1997), and sigma-1 receptors (343 nM) (Narita et al., 1996). This indicates that the sigma-1 interaction may have a larger role in producing the antidepressant-like effects of DM than that of imipramine. This is consistent with the wider range of protein targets through which imipramine, but not DM, interacts, which include: 5- $\mathrm{HT}_{2}$, muscarinic, and histamine $\mathrm{H}_{1}$ receptors (Andersen, 1989; Cusack et al., 1994; Stanton et al., 1993; Tran et al., 1978; Werling et al., 2007a; Wong et al., 1982). Moreover, BD1063 failed to significantly attenuate the antidepressant-like effects of ketamine, which is in accordance with a previous in vivo study (Robson et al., 2012). As sigma-1 receptors have been implicated in mediating the in vitro effects of ketamine on potentiating neurite outgrowth, however, it may be that sigma-1 receptors play a more prominent role in the sustained, rather than acute effects of ketamine observed clinically (Robson et al., 2012). DM, with higher binding affinity to sigma-1 receptors (Chou et al., 1999; Fishback et al., 2012; Werling et al., 2007a), may convey additional therapeutic advantages over ketamine under clinically relevant conditions since ketamine has only micromolar affinity for these receptors (Robson et al., 2012). 
Finally, DM and ketamine elicit stimulant actions which were quantified herein as increases in locomotor activity in the OFT. For DM, the stimulant effects cannot account for the antidepressant-like actions of DM: DM at both $1 \mathrm{mg} / \mathrm{kg}$ and $30 \mathrm{~m} / \mathrm{kg}$ had significant stimulatory effects whereas only DM at $30 \mathrm{mg} / \mathrm{kg}$ had antidepressant-like effects. Moreover, a correlation analysis using Pearson's r correlation test confirmed that there was no correlation between the DM-induced increase in locomotor activity and decrease in immobility time for all the doses tested. We also found that quinidine enhances the antidepressant-like effects of DM without producing an increase in locomotor activity. This suggests that addition of quinidine to DM can be used clinically to optimize therapeutic antidepressant actions, without eliciting unwanted stimulant effects. With regards to ketamine, there was a dose-dependent decrease in immobility time as well as increase in locomotor activity, and only the highest dose of ketamine tested (30 $\mathrm{mg} / \mathrm{kg}$ ) produced both stimulatory and antidepressant-like effects. This indicates that these behavioral responses to ketamine may be an all-or-none phenomenon and may have confounded data interpretation. In depressed patients treated with ketamine, significant relationships between ketamine's rapid antidepressant and acute psychotomimetic effects have also been observed (Luckenbaugh et al., 2014; Sos et al., 2013). These CNS effects are likely mediated by activation of common mediators of both antidepressant and psychostimulant responses vs. producing antidepressant actions secondary to its ability to produce psychostimulant effects. Supporting the former scenario, ketamine has been shown to produce rapid clinical antidepressant effects without inducing psychotomimetic effects in some human studies (Lapidus et al., 2014; Valentine et al., 2011), and a slightly higher dose of ketamine (40 mg/kg) than used here has been shown to exhibit antidepressant-like effects in the FST without affecting locomotor activity in the OFT under the same experimental conditions (Robson et al., 2012). Importantly, ketamine elicits much greater stimulatory effects than DM at the antidepressant effective dose (2868 \pm 426 vs $1515 \pm$ 228), supporting that DM may elicit antidepressant efficacy with less abuse liability and untoward side effects.

In conclusion, the data presented here show for the first time that DM has antidepressant-like effects in an in vivo model and provide evidence that this effect occurs at least in part through a sigma-1 
receptor-dependent mechanism. This is also the first report of the manner in which DM interacts at the sigma-1 receptor. Together with earlier studies and the potential of increasing DM bioavailability by using the FDA- and EMA-approved DM/quinidine formulation, these data suggest DM should be further explored for translational potential as an antidepressant drug in clinical trials, as it may offer rapid acting relief of depressive symptoms and the ability to resolve cases of TRD. In addition, further studies to understand the molecular and cellular mechanisms by which these effects occur are necessary and may yield important information about how various receptors, transporters and processes are involved in the ability of DM to convey its antidepressant effects.

Acknowledgments: The contributions of Matthew Robson, Jason Healy, Anna Scandinaro and Ying Huang Zhang is acknowledged. Funding was provided by Avanir Pharmaceuticals, West Virginia University, and the National Institutes of Health (DA013978, DA013583). Portions of this chapter were published in part or in full as: Nguyen, L., Robson, M.J., Healy, J.R., Scandinaro, A.L., and Matsumoto, R.R. (2014). Involvement of sigma-1 receptors in the antidepressant-like effects of dextromethorphan. PloS one 9, e89985. 


\section{CHAPTER 4}

Involvement of AMPA receptors in the antidepressant-like effects of DM 


\subsection{Introduction}

In the previous chapter, we found that DM exerts antidepressant-like effects in the FST, the most validated animal model for predicting antidepressant efficacy (Cryan and Holmes, 2005; McArthur and Borsini, 2006; Nestler et al., 2002b). In the present chapter, we investigated whether DM also has antidepressant-like actions in the TST, which is an additional widely used behavioral assay for assessing antidepressant potential (Cryan et al., 2005), to confirm the antidepressant-like effects of DM. Moreover, because DM undergoes substantial first-pass metabolism by CYP2D6 to its major active metabolite DX (Schmid et al., 1985), quinidine was administered concomitantly with DM to raise the plasma concentration and bioavailability of DM (Pope et al., 2004) and to begin examining whether the metabolism of DM affects its antidepressant efficacy in the TST. Finally, in view of the possible involvement of AMPA receptors in antidepressant actions, using both the TST and FST, an AMPA receptor antagonist (NBQX), was evaluated in conjunction with DM to determine the role of AMPA receptors in the antidepressant actions of DM. Conventional and fast acting antidepressants represented by imipramine and ketamine, respectively, were used as reference ligands in these behavioral assays.

\subsection{Results}

4.2.1. Antidepressant-like effects of imipramine, ketamine, and DM in the TST

The conventional antidepressant drug imipramine and fast acting antidepressant ketamine served as positive controls and both reduced immobility time in the TST (Figure 8A and B). ANOVA confirmed a significant difference between the behavioral changes produced by the various doses of imipramine $(\mathrm{F}[2,33]=5.06, \mathrm{P}<0.05)$ and ketamine $(\mathrm{F}[3,38]=9.02, \mathrm{P}<0.001)$. Post-hoc Dunnett's tests showed imipramine at $40 \mathrm{mg} / \mathrm{kg}$ and ketamine at $30 \mathrm{mg} / \mathrm{kg}$ significantly decreased immobility time when compared to saline ( $\mathrm{q}=3.14, \mathrm{P}<0.01$ and $\mathrm{q}=5.08, \mathrm{P}<0.001$, respectively). Similar to the reference ligands, DM significantly reduced immobility time (Figure $8 \mathrm{C} ; \mathrm{F}[3,43]=28.28, \mathrm{P}<0.0001$ ), with a post-hoc Dunnett's test revealing that the dose of $30 \mathrm{mg} / \mathrm{kg}$ differed significantly from saline $(\mathrm{q}=7.70, \mathrm{P}<0.001)$. 
Figure 8. Antidepressant-like effects of imiprame (A), ketamine (B), and DM (C) in the TST. Data are expressed as mean \pm SEM. $* * P<0.01$, $* * * \mathrm{P}<0.001$ vs. saline-treated group $(0 \mathrm{mg} / \mathrm{kg}$, i.p.); one-way ANOVA followed by post-hoc Dunnett's tests.
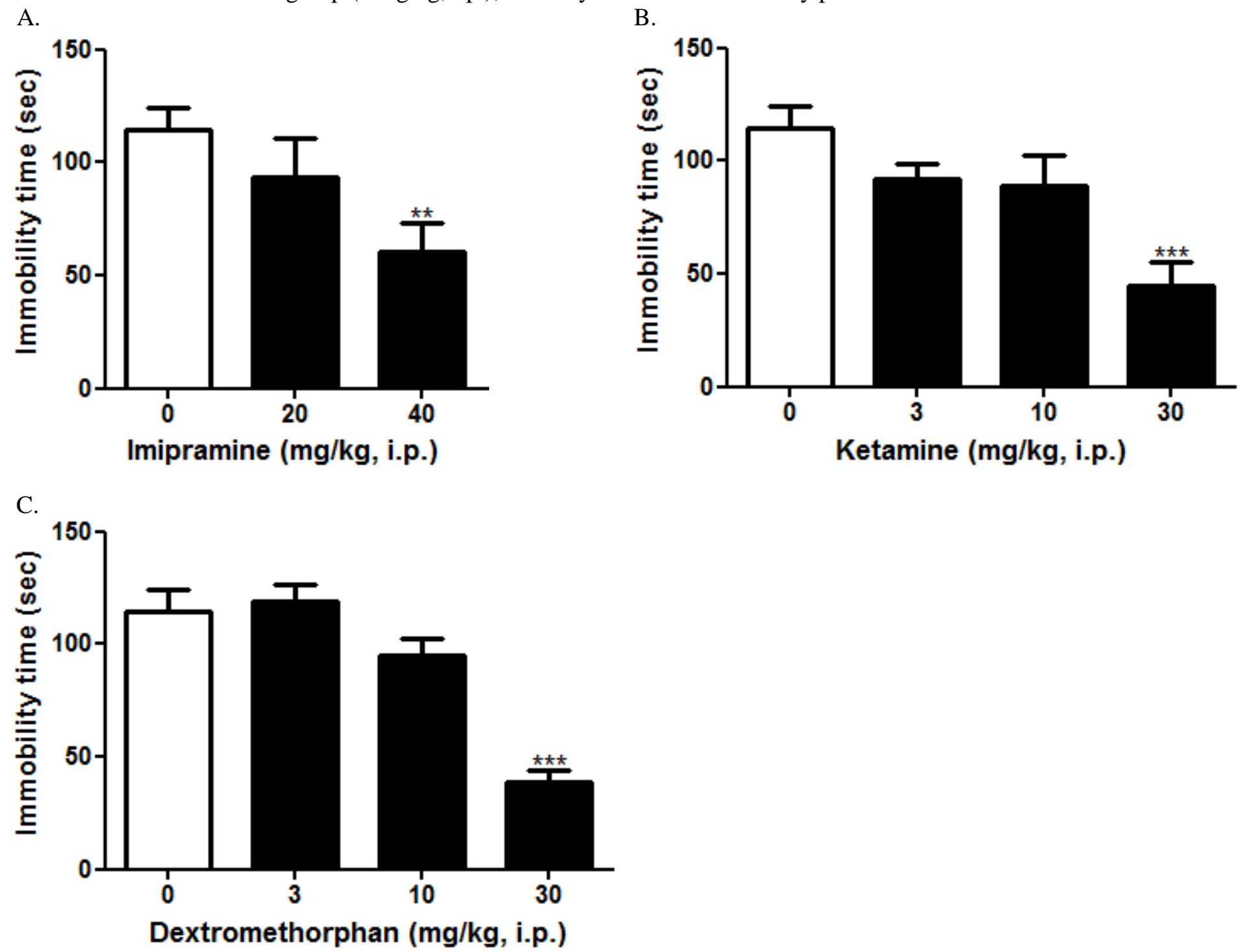
4.2.2. CYPD2D6 inhibition potentiates the antidepressant-like effects of DM in the TST

Concurrent administration of quinidine $(30 \mathrm{mg} / \mathrm{kg})$ potentiated the antidepressant-like effects of DM (10 mg/kg) (Figure 9). ANOVA confirmed a significant difference between the various treatment groups $(\mathrm{F}[5,58]=19.74, \mathrm{P}<0.0001)$. Post-hoc Tukey's multiple comparison tests showed that $\mathrm{DM}$ at 30 $\mathrm{mg} / \mathrm{kg}$ differed significantly from saline in the absence of quinidine $(\mathrm{q}=10.34, \mathrm{P}<0.001)$ and in combination with quinidine ( $\mathrm{q}=9.32, \mathrm{P}<0.001)$. Importantly, while $\mathrm{DM}$ at $10 \mathrm{mg} / \mathrm{kg}$ in the absence of quinidine was not significantly different from saline (q=2.37, n.s.), it produced a significant decrease in immobility time when combined with quinidine $(\mathrm{q}=5.35, \mathrm{P}<0.01)$. When tested alone, quinidine had no significant effects in the TST (t=0.28, n.s.).

4.2.3. AMPA receptor antagonist attenuates the antidepressant-like effects of imipramine, ketamine, and DM in the TST

Pretreatment of mice with behaviorally inactive doses of NBQX (10 or $30 \mathrm{mg} / \mathrm{kg}$ ) attenuated the antidepressant-like effect of ketamine, DM, and imipramine (Figure 10). ANOVA confirmed a significant difference between the various treatment groups in the antagonism study for ketamine (Figure 10A; $\mathrm{F}[5,59]=9.23, \mathrm{P}<0.0001$ ) and DM (Figure 10B; F[5,60]=20.27, P<0.0001). Post-hoc Tukey's multiple comparison tests revealed that the ketamine alone treatment group differed significantly from saline $(\mathrm{q}=6.37, \mathrm{P}<0.001)$, as well as the NBQX $(30 \mathrm{mg} / \mathrm{kg})+$ Ketamine group $(\mathrm{q}=6.60, \mathrm{P}<0.001)$. Similarly, post-hoc Tukey's tests confirmed that the DM alone treatment group also differed from saline (q=8.92, $\mathrm{P}<0.001)$, as well as the NBQX $(30 \mathrm{mg} / \mathrm{kg})+\mathrm{DM}$ group $(\mathrm{q}=4.37, \mathrm{P}<0.05)$. The reduction in immobility time induced by imipramine was also significantly prevented by NBQX (Figure 10C; F[3,48]=6.50, $\mathrm{P}<0.001)$. Post-hoc Tukey's test confirmed that NBQX $(30 \mathrm{mg} / \mathrm{kg})$ pretreatment attenuated the ability of imipramine to decrease immobility time $(\mathrm{q}=4.163, \mathrm{P}<0.05)$. Although not shown in the figure, NBQX alone had no significant effects on immobility time in the TST at 10,20 or $30 \mathrm{mg} / \mathrm{kg}(\mathrm{F}[3,38]=1.89$, n.s. $)$. 
Figure 9. Potentiation of the antidepressant-like effects of DM by quinidine in the TST. Data are expressed as mean \pm SEM. $* * \mathrm{P}<0.01$, $* * * \mathrm{P}<0.001$ vs. saline-treated group; one-way ANOVA followed by post-hoc Tukey's tests. Black bar ( $\square$ ) with quinidine $(30 \mathrm{mg} / \mathrm{kg})$. White bar ( $\square$ ) without quinidine (30 $\mathrm{mg} / \mathrm{kg}$ ).

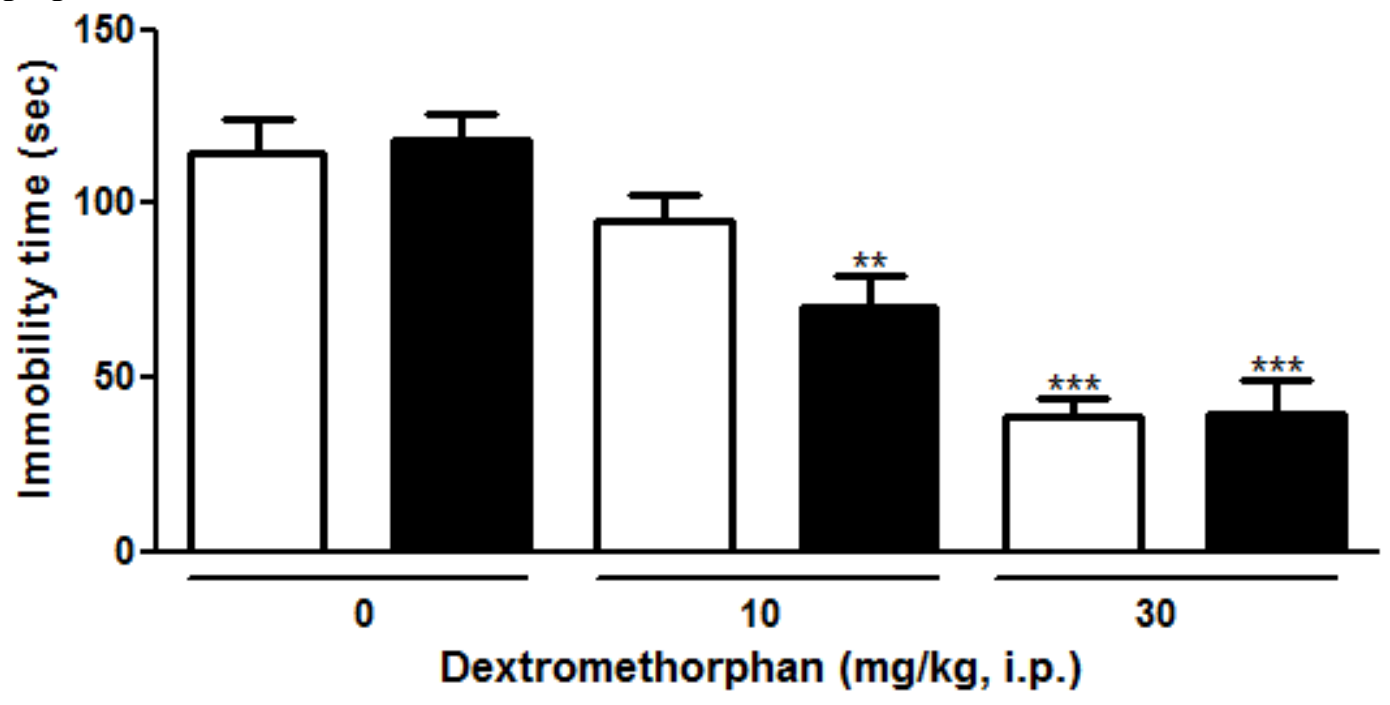

Figure 10. Blockade of AMPA receptors prevented the antidepressant-like effects of ketamine (A), DM (B), and imipramine (C) in the TST. Data are expressed as mean \pm SEM. $* \mathrm{P}<0.05$, $* * * \mathrm{P}<0.001$ vs. salinetreated group; \#P<0.05 vs. DM- or impramine-treated group, \#\#\# $<0.001$ vs ketamine-treated group; oneway ANOVA followed by post-hoc Tukey's tests. KET, ketamine. DM, dextromethorphan. IM, imipramine.

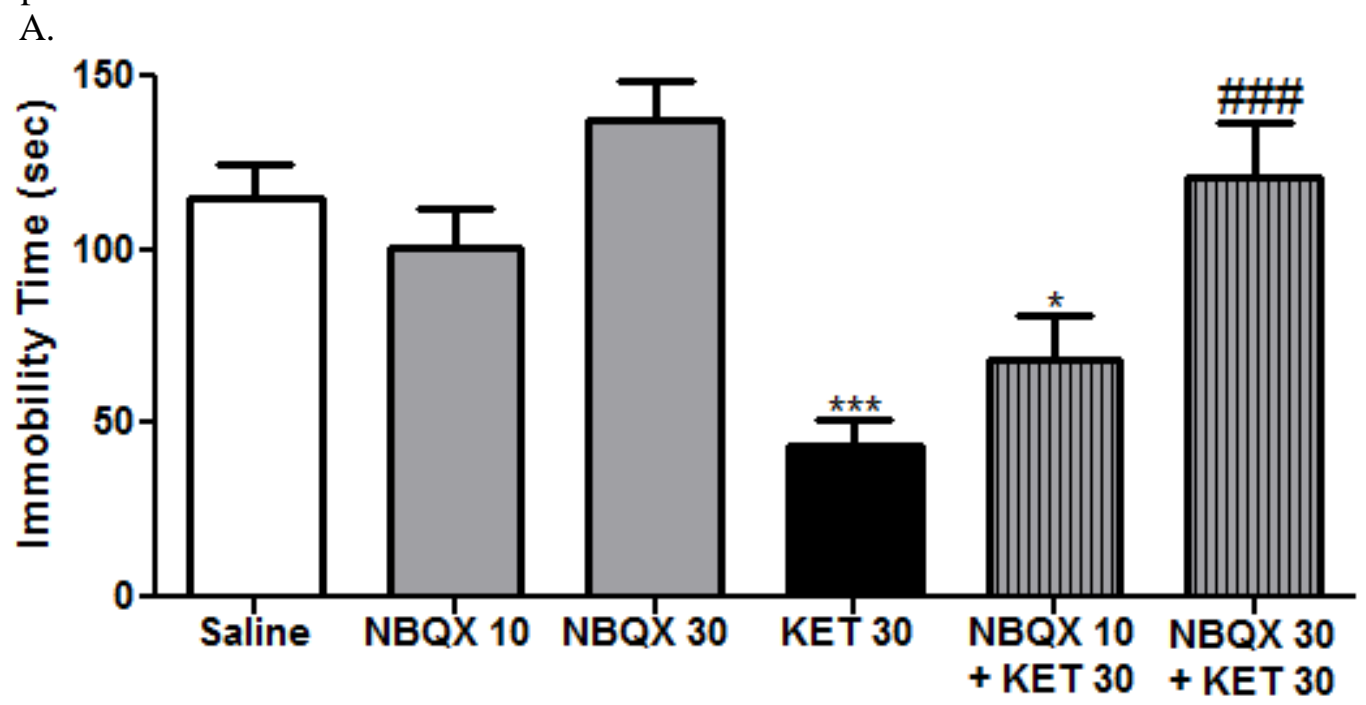


B.

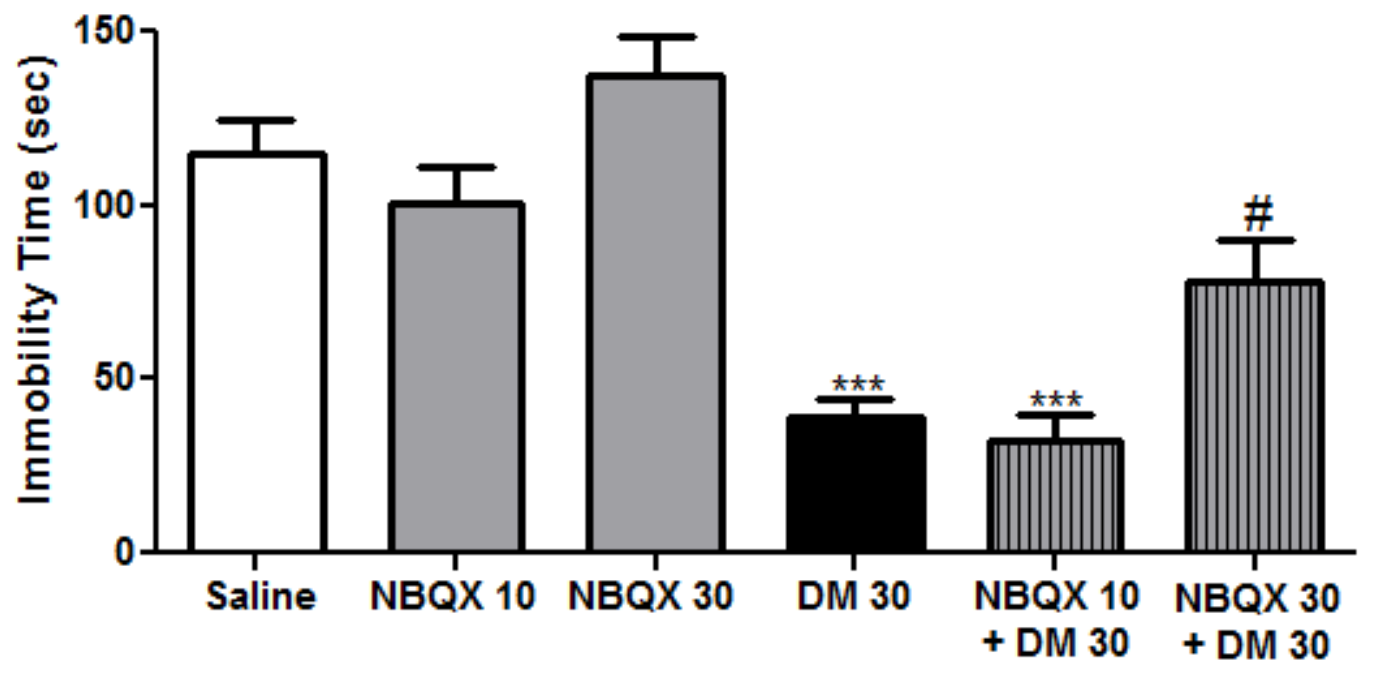

C.

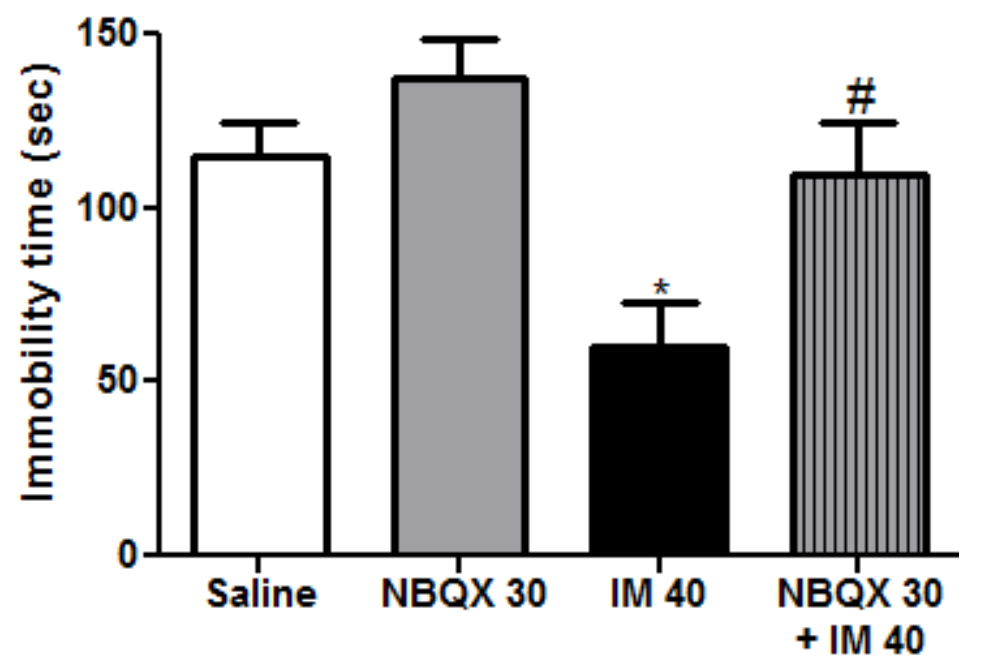


4.2.4. Effects of AMPA receptor antagonist on the antidepressant-like effects of imipramine, ketamine, and DM in the FST

In the FST, behaviorally inactive doses of NBQX were administered in conjunction with previously established antidepressant effective doses of the compounds of interest (Autry et al., 2011; Robson et al., 2012). Pretreatment of mice with NBQX (10 or $30 \mathrm{mg} / \mathrm{kg}$ ) attenuated the antidepressantlike effects of ketamine and DM, but not that of imipramine (Figure 11). ANOVA confirmed a significant difference between the various treatment groups in the antagonism studies for ketamine (Figure 11A; $\mathrm{F}[5,57]=10.44, \mathrm{P}<0.0001)$. Post-hoc Tukey's tests revealed that the ketamine alone treatment group differed significantly from saline $(\mathrm{q}=6.71, \mathrm{P}<0.001)$, as well as the NBQX $(30 \mathrm{mg} / \mathrm{kg})+$ Ketamine group $(\mathrm{q}=5.51, \mathrm{P}<0.01)$. Similar to the mitigation of the antidepressant-like effects of ketamine, pretreatment with NBQX (10 mg/kg) attenuated the antidepressant-like effects of DM (Figure 11B). ANOVA confirmed a significant difference between the various treatment groups in the antagonism study for DM $(\mathrm{F}[3,41]=11.30, \mathrm{P}<0.0001)$. Post-hoc Tukey's multiple comparison test revealed that the DM alone treatment group differed significantly from saline $(\mathrm{q}=7.01, \mathrm{P}<0.001)$, as well as the NBQX + DM group $(\mathrm{q}=3.80, \mathrm{P}<0.05)$. In contrast to the attenuation of the ketamine-induced effects in the FST, pretreatment with NBQX (30 mg/kg) failed to prevent the antidepressant-like effects of imipramine (Figure 11C). ANOVA confirmed a significant difference between the various treatment groups in the antagonism study for imipramine $(\mathrm{F}[3,36]=24.00, \mathrm{P}<0.0001)$. Post-hoc Tukey's test confirmed that imipramine significantly reduced immobility time compared to saline $(\mathrm{q}=6.94, \mathrm{P}<0.001)$. However, post-hoc comparisons of the NBQX + Imipramine group showed that its effects did not differ significantly from imipramine alone ( $\mathrm{q}=1.14$, n.s.), and there was a significant reduction in immobility time compared to saline ( $\mathrm{q}=8.08$, $\mathrm{P}<0.001)$. When tested alone, NBQX at 10 or $30 \mathrm{mg} / \mathrm{kg}$ did not significantly affect the immobility time in the FST $(\mathrm{F}[2,27]=1.34$, n.s. $)$.

\subsubsection{Effects of the drug treatments on locomotor activity}

Combining the locomotor activity data for all the animals used in this study (TST and FST), the 
Figure 11. Blockade of AMPA receptors prevented the antidepressant-like effects of ketamine (A) and DM (B), but not of imipramine (C) in the FST. Data are expressed as mean \pm SEM. $* \mathrm{P}<0.05, * * * \mathrm{P}<0.001$ vs. saline-treated group; \#P<0.05 vs. DM-treated group, \#\#P<0.01 vs ketamine-treated group; one-way ANOVA followed by post-hoc Tukey's tests. KET, ketamine. DM, dextromethorphan. IM, imipramine.
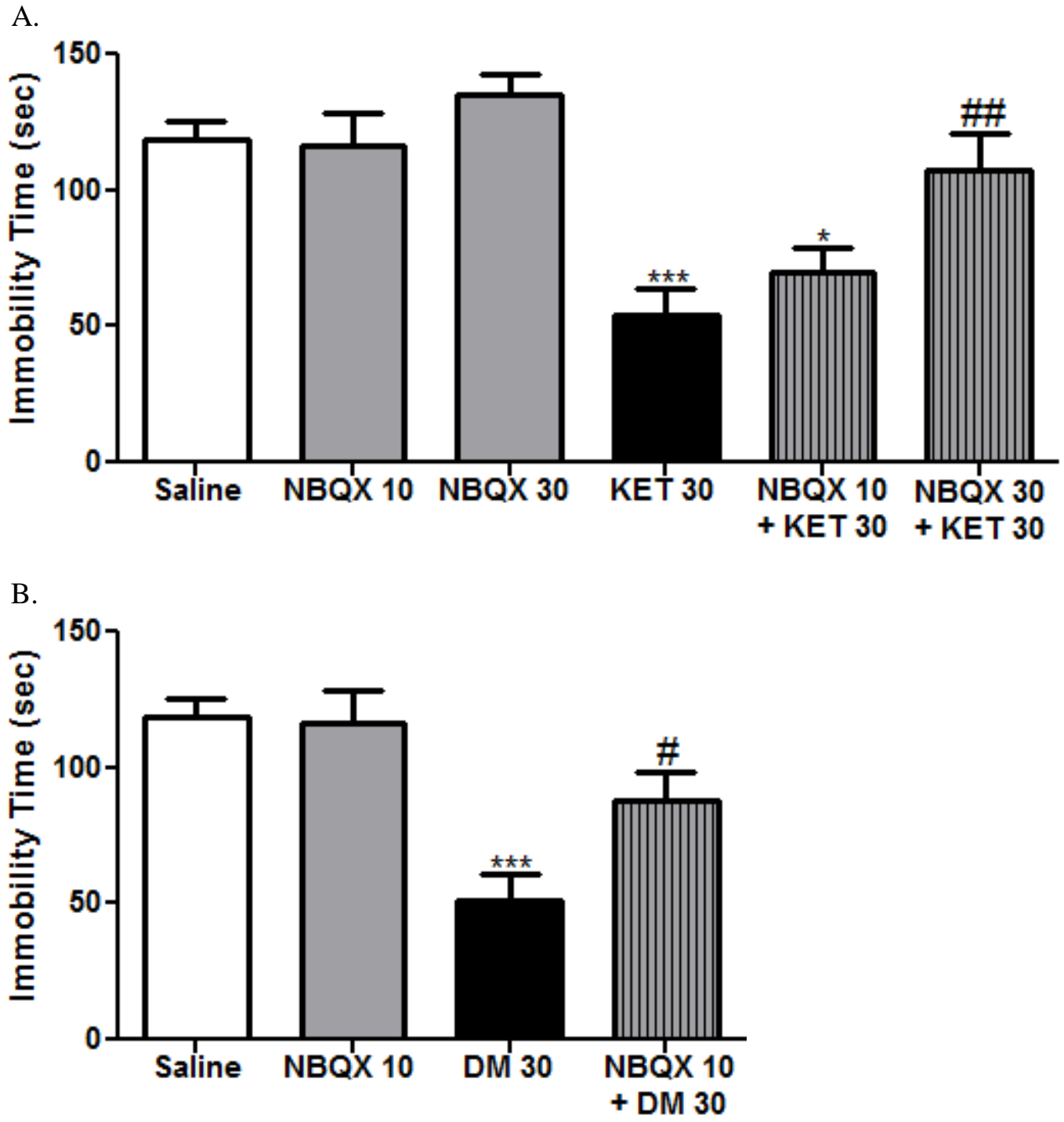


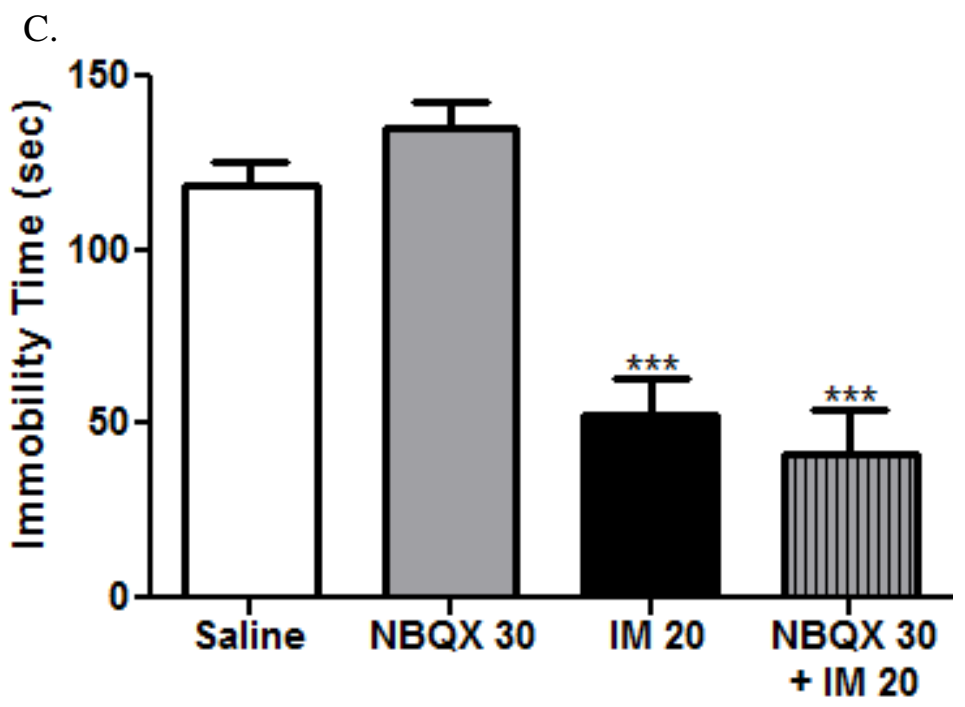


effects of the various drug treatments in the OFT are shown in Table 4. Ketamine produced a significant increase in locomotor activity $(\mathrm{F}[3,70]=13.97, \mathrm{P}<0.001)$ while imipramine produced a significant decrease $(F[2,54]=16.68, P<0.001)$. DM did not significantly influence locomotor activity $(F[3,63]=1.75$, n.s.). Quinidine had no significant effects alone ( $\mathrm{t}=0.17$, n.s.), or in combination with $\mathrm{DM}(\mathrm{F}[5,70]=2.22$, n.s.). $\mathrm{NBQX}$ alone had no significant effects $(\mathrm{F}[3,68]=0.65$, n.s. $)$, but in combination with $\mathrm{DM}$ or imipramine produced significant decreases in locomotor activity $(\mathrm{F}[5,116]=4.82, \mathrm{P}<0.001$ and $\mathrm{F}[5,97]=17.28, \mathrm{P}<0.001$, respectively). NBQX pretreatment also mitigated the stimulatory effects of ketamine $(\mathrm{F}[5,125]=19.29, \mathrm{P}<0.001)$.

Since ketamine produced significant stimulant effects, a correlation analysis between locomotor activity and immobility time was carried out to determine whether the stimulant effects of ketamine were related to its apparent antidepressant-like behaviors. The Pearson's r correlation test including data points from all the doses of ketamine tested revealed a significant inverse relationship between the ketamineinduced alterations in locomotor activity and immobility time in the TST and FST $(r=-0.34, \mathrm{P}<0.05)$.

\subsection{Discussion}

Extending and corroborating our previous findings with the FST (Nguyen et al., 2014), this is the first study that demonstrates the antidepressant-like effects of DM in the TST, in addition to implicating AMPA receptors as a critical element in mediating the antidepressant-like actions of DM. Moreover, similar to the findings in the FST (Nguyen et al., 2014), concomitant administration of the CYP2D6 inhibitor quinidine potentiated the antidepressant effects of DM in the TST, lending further support that the antidepressant-like properties of DM may be enhanced with increased early exposure of the parent compound compared to the major metabolites.

We also demonstrated that AMPA receptors play an important role in the antidepressant effects of ketamine in the two behavioral assays and that AMPA receptors are not involved in the antidepressant actions of imipramine in the FST, as previously described by others (Autry et al., 2011; Koike et al., 2011; Li et al., 2001; Maeng et al., 2008; Wolak et al., 2013). Interestingly, we found that in the TST, 
Table 4. Effects of various treatments on locomotor activity in the OFT. Data are expressed as mean \pm SEM. $* \mathrm{P}<0.05$, $* * \mathrm{P}<0.01$, $* * * \mathrm{P}<0.001$ vs. saline-treated group; \#\#\#P<0.001 vs. ketamine- or DM-treated group; one-way ANOVA followed by post-hoc Dunnett's or Tukey's tests. KET, ketamine. DM, dextromethorphan. IM, imipramine. NBQX, 2,3-dioxo-6-nitro-1,2,3,4-tetrahydrobenzo[f]quinoxaline-7sulfonamide. QND, quinidine. n.s., not significant.

\begin{tabular}{|c|c|c|c|}
\hline $\begin{array}{c}\text { Treatment and dose } \\
(\mathrm{mg} / \mathrm{kg}, \text { i.p. })\end{array}$ & $\begin{array}{c}\text { Locomotor activity } \\
\text { (counts) }\end{array}$ & Statistical analysis & $\begin{array}{l}\text { Post-hoc Dunnett's or } \\
\text { Tukey's, if applicable }\end{array}$ \\
\hline \multicolumn{4}{|l|}{ Saline } \\
\hline Saline & $1129 \pm 112$ & & \\
\hline \multicolumn{4}{|l|}{ Ketamine } \\
\hline KET 3 & $1583 \pm 314$ & \multirow{3}{*}{$\begin{array}{l}\text { ANOVA F }(3,70)= \\
13.97 \\
\mathrm{P}<0.001\end{array}$} & $\mathrm{q}=1.07$, n.s. vs. saline \\
\hline KET 10 & $1617 \pm 179$ & & $\mathrm{q}=1.30$, n.s. vs. saline \\
\hline KET 30 & $2964 \pm 268 * * *$ & & $\mathrm{q}=5.11, \mathrm{P}<0.001$ vs. saline \\
\hline \multicolumn{4}{|l|}{ Dextromethorphan } \\
\hline DM 3 & $1441 \pm 249$ & \multirow{3}{*}{$\begin{array}{l}\text { ANOVA F }(3,63)= \\
1.75 \\
\text { n.s. }\end{array}$} & \\
\hline DM 10 & $1649 \pm 161$ & & \\
\hline DM 30 & $1262 \pm 139$ & & \\
\hline \multicolumn{4}{|l|}{ Imipramine } \\
\hline IM 20 & $496 \pm 79 * * *$ & \multirow{3}{*}{$\begin{array}{l}\text { ANOVA F }(2,54)= \\
16.68 \\
\mathrm{P}<0.001\end{array}$} & $\mathrm{q}=5.12, \mathrm{P}<0.001$ vs. saline \\
\hline IM 40 & $505 \pm 49 * * *$ & & $\mathrm{q}=4.85, \mathrm{P}<0.001$ vs. saline \\
\hline \multicolumn{3}{|l|}{ CYP2D6 Inhibition } & \\
\hline \multicolumn{4}{|l|}{ Quinidine + Saline } \\
\hline QND $30+$ Saline & $1095 \pm 164$ & $\begin{array}{l}\text { Unpaired t test } \\
\text { n.s. }\end{array}$ & \\
\hline \multicolumn{4}{|l|}{ Quinidine + Dextromethorphan } \\
\hline QND $30+$ Saline & $1095 \pm 164$ & \multirow{5}{*}{$\begin{array}{l}\text { ANOVA } F(5,70)=2.22 \\
\text { n.s. }\end{array}$} & \\
\hline DM 10 & $1649 \pm 161$ & & \\
\hline QND 30 + DM 10 & $854 \pm 117$ & & \\
\hline DM 30 & $1262 \pm 139$ & & \\
\hline QND $30+$ DM 30 & $1262 \pm 275$ & & \\
\hline \multicolumn{4}{|l|}{ AMPA Antagonism } \\
\hline \multicolumn{4}{|l|}{ NBQX } \\
\hline NBQX 10 & $1208 \pm 170$ & \multirow{3}{*}{$\begin{array}{l}\text { ANOVA } F(3,69)=0.65 \\
\text { n.s. }\end{array}$} & \\
\hline NBQX 20 & $1327 \pm 270$ & & \\
\hline NBQX 30 & $993 \pm 141$ & & \\
\hline \multicolumn{4}{|l|}{ NBQX + Ketamine } \\
\hline NBQX 10 & $1208 \pm 170$ & \multirow{5}{*}{$\begin{array}{l}\text { ANOVA } \mathrm{F}(5,125)= \\
19.29 \\
\mathrm{P}<0.001\end{array}$} & $\mathrm{q}=0.3730$, n.s. vs. saline \\
\hline NBQX 30 & $993 \pm 141$ & & $\mathrm{q}=0.6365$, n.s. vs. saline \\
\hline KET 30 & $2964 \pm 268^{* * *}$ & & $\mathrm{q}=9.454, \mathrm{P}<0.001$ vs. saline \\
\hline NBQX $10+$ KET 30 & $2176 \pm 290 *$ & & $\begin{array}{l}\mathrm{q}=4.837, \mathrm{P}<0.05 \text { vs. saline; } \\
\mathrm{q}=3.885, \text { n.s. vs. KET } 30\end{array}$ \\
\hline NBQX $30+$ KET 30 & $735 \pm 105 \# \# \#$ & & $\begin{array}{l}\mathrm{q}=1.840, \text { n.s. vs. saline; } \\
\mathrm{q}=11.16, \mathrm{P}<0.001 \text { vs. KET } 30\end{array}$ \\
\hline \multicolumn{4}{|l|}{ NBQX + Dextromethorphan } \\
\hline NBQX 10 & $1208 \pm 170$ & \multirow{5}{*}{$\begin{array}{l}\text { ANOVA F }(5,116)= \\
4.82 \\
\mathrm{P}<0.001\end{array}$} & $\mathrm{q}=0.57$, n.s. vs. saline \\
\hline NBQX 30 & $993 \pm 141$ & & $\mathrm{q}=0.97$, n.s. vs. saline \\
\hline DM 30 & $1340 \pm 141$ & & $\mathrm{q}=1.66$, n.s. vs. saline \\
\hline NBQX $10+$ DM 30 & $1559 \pm 131$ & & $\begin{array}{l}\mathrm{q}=0.21, \text { n.s. vs. saline; } \mathrm{q}=1.38 \text {, } \\
\text { n.s. vs. DM } 30\end{array}$ \\
\hline NBQX $30+$ DM 30 & $221 \pm 28 * *$,\#\#\# & & $\begin{array}{l}\mathrm{q}=5.22, \mathrm{P}<0.01 \text { vs. saline; } \\
\mathrm{q}=6.73, \mathrm{P}<0.001 \text { vs. } \mathrm{DM} 30\end{array}$ \\
\hline
\end{tabular}




\begin{tabular}{|c|c|c|c|}
\hline \multicolumn{4}{|l|}{ NBQX + Imipramine } \\
\hline NBQX 30 & $993 \pm 141$ & \multirow{5}{*}{$\begin{array}{l}\text { ANOVA } \\
F(5,97)=17.28 \\
P<0.001\end{array}$} & $\mathrm{q}=1.51$, n.s. vs. saline \\
\hline IM 20 & $496 \pm 79 * * *$ & & $\mathrm{q}=7.01, \mathrm{P}<0.001$ vs. saline \\
\hline IM 40 & $505 \pm 49$ *** & & $\mathrm{q}=6.38, \mathrm{P}<0.001$ vs. saline \\
\hline NBQX $30+$ IM 20 & $135 \pm 23 * * *$ & & $\begin{array}{l}\mathrm{q}=8.92, \mathrm{P}<0.001 \text { vs. saline; } \\
\mathrm{q}=3.19, \text { n.s. vs. IM } 20\end{array}$ \\
\hline NBQX $30+$ IM 40 & $150 \pm 26^{* * *}$ & & $\begin{array}{l}\mathrm{q}=4.89, \mathrm{P}<0.001 \text { vs. saline, } \\
\mathrm{q}=3.32 \text {, n.s. vs. IM } 40\end{array}$ \\
\hline
\end{tabular}


however, AMPA receptors appear to contribute to the effects of imipramine. This is to our knowledge the first published report of the ability of an AMPA receptor antagonist to attenuate the acute antidepressantlike effects of a conventional antidepressant. We cannot rule out the possibility that because NBQX significantly decreased locomotor activity in combination with imipramine, NBQX may have functionally "reversed" the antidepressant-like effect of imipramine in the TST. Nevertheless, despite also significantly decreasing locomotor counts in the cohort of animals subjected to the FST $(135 \pm 23$ in FST cohort vs. $150 \pm 26$ in TST cohort), NBQX did not functionally reverse the antidepressant-like effects of imipramine in the FST. The discrepancy between the TST and FST under our experimental conditions may be due to a difference in neural circuitry responsible for the observed behaviors in these two tests (Cryan et al., 2005), and this may be specific to certain mouse strains (Bai et al., 2001; Lucki et al., 2001; Sugimoto et al., 2008; Vaugeois et al., 1997). In C57BL/6 mice, for example, imipramine produced a linear dose-responsive curve in the TST but a biphasic (U-shaped) curve in the FST (Bai et al., 2001). Moreover, in the same study, imipramine caused a linear dose-responsive curve in the FST in NIH Swiss mice, supporting that not only are there inherent intra-strain differences in the biology of the FST and TST but also significant inter-strain differences in the same test. Of note, Li et al. found that administration of LY392098 (an AMPA receptor potentiator) produced a significant enhancement in the potency of imipramine, and likewise an ineffective dose of imipramine potentiated the antidepressant-like effects of LY392098 (Li et al., 2003). Additional studies are needed to determine how imipramine and other monoaminergic-based antidepressants may work alternatively or in concert with AMPA receptor activation to produce antidepressant responses, and whether AMPA receptors play similar functions in mediating the effects of conventional vs. rapid acting antidepressant drugs. Nevertheless, the present findings are consistent with the mounting evidence of the important contributory role AMPA receptors may have in the therapeutic activity of various antidepressant drugs (Alt et al., 2006; Alt et al., 2005; Bleakman et al., 2007).

In light of the finding that NBQX pretreatment significantly attenuated the antidepressant-like behaviors of DM in two rodent models predictive of antidepressant efficacy and of imipramine in only 
one of the two, AMPA receptors appear to play a greater role in mediating the antidepressant actions of DM than that of imipramine. The difference in doses of NBQX needed to significantly mitigate the effects of DM in the TST and FST may, like the discrepant imipramine data, relate to differences in neural circuitry involved in these two tests, especially for a drug such as DM with multiple possible mechanisms of antidepressant action. The ability of DM to elicit antidepressant-like effects through AMPA receptors similar to that of ketamine warrants future studies to evaluate its potential fast acting antidepressant effects, for AMPA receptor activation may contribute to a faster onset of antidepressant efficacy (Adell et al., 2005; Duman, 2014b). For example, AMPA receptor activation has been shown to increase BDNF expression in vitro and vivo (Dicou et al., 2003; Jourdi et al., 2009; Lauterborn et al., 2000; Lauterborn et al., 2009; Lauterborn et al., 2003; Mackowiak et al., 2002), a pivotal downstream component for conveying rapid acting and delayed therapeutic efficacy (Castren and Rantamaki, 2010; Duman and Voleti, 2012). Importantly, a single injection with an AMPA receptor potentiator increased BDNF mRNA expression in the hippocampus within hours (Mackowiak et al., 2002) compared to the more chronic dosing usually required of traditional antidepressants (Duman and Monteggia, 2006). In addition, Knapp et al. reported that an AMPA receptor potentiator produces a more rapid onset of action than the conventional antidepressant fluoxetine in the reduction of submissive behavior paradigm (Knapp et al., 2002), a recently developed rodent model for assessing antidepressant drug activity (Malatynska et al., 2002; Malatynska et al., 2005). Several studies have also shown that pretreatment with an AMPA receptor antagonist blocks the antidepressant-like behavioral actions of ketamine and scopolamine (Drevets and Furey, 2010; Furey and Drevets, 2006; Koike et al., 2011; Maeng et al., 2008; Voleti et al., 2013), two clinically validated fast acting antidepressants (Berman et al., 2000; Drevets and Furey, 2010; Furey and Drevets, 2006; Ghasemi et al., 2014; Lara et al., 2013; Murrough et al., 2013a; Price et al., 2009; Zarate et al., 2006), in addition to some of the neural plasticity-related biochemical changes induced by ketamine (Maeng et al., 2008; Zhou et al., 2014). Further work is required to determine if DM may also influence these pathways and targets, and produce a fast antidepressant response in models like learned helplessness and chronic unpredictable stress. 
It remains unknown how DM may alter AMPA receptor activity, as it does not directly bind to AMPA receptors (Lauterbach, 2012). The activation of AMPA is hypothesized to occur downstream from binding to initial targets, as has been reported for ketamine which also appears to not directly affect AMPA receptors (Jin, 2007; Lu and Bieger, 1996). In addition, DM has significant affinity for sigma-1 receptors (Fishback et al., 2012). We previously found that DM elicits antidepressant-like actions at least in part through sigma-1 receptors (Nguyen et al., 2014), a protein target for a potential new class of antidepressant drugs (Fishback et al., 2010) that, similar to AMPA receptors, may contribute to a more rapid onset of antidepressant efficacy (Hayashi and $\mathrm{Su}, 2008$ ). Sigma-1 ligands have been shown to regulate AMPA mRNA and protein expression levels (Liang and Wang, 1998) and modulate AMPA receptor neurotransmission (Guitart et al., 2000). Consequently, one indirect mechanism by which DM could activate AMPA receptors is through the activation of sigma-1 receptors to elicit an antidepressant response.

Finally, in this study cohort, we found that DM had no effects on locomotor activity, in contrast to our recent previous report of stimulatory effects (Nguyen et al., 2014). Since the same laboratory conditions and methods were applied to these two studies, this incongruity may be due to the heterogeneous genetic background inherent to outbred mice. Supporting this, our previous saline-treated cohort of mice had nearly a $40 \%$ lower count of locomotor activity than the present saline-treated group $(688 \pm 61$ (Nguyen et al., 2014) vs. $1129 \pm 112)$.

Unlike DM, ketamine had significant stimulatory effects. In particular, only the highest dose of ketamine tested $(30 \mathrm{mg} / \mathrm{kg}$ ) produced both stimulatory and antidepressant-like effects, suggesting that these behavioral responses to ketamine may be an all-or-none phenomenon and may have confounded data interpretation. Moreover, the correlation analysis revealed a significant relationship between these behavioral actions of ketamine. Since correlation does not equal causation, however, it is unclear whether the ability of ketamine to elicit antidepressant-like actions is secondary to its ability to produce stimulant effects or whether ketamine is capable of activating common mediators of both the antidepressant and stimulant responses. In support of the latter scenario, our lab has previously shown in a separate cohort of 
animals, ketamine (40 mg/kg) elicited antidepressant-like effects in the FST without incurring effects on locomotor activity (Robson et al., 2012). Regardless, the fact that ketamine had significant stimulatory effects and DM did not in the current cohort of mice further supports that DM may elicit antidepressant efficacy with less abuse liability and untoward side effects. Noteworthy, DM in combination with quinidine has been reported in depressed patients with treatment-resistant bipolar disorder to have some rapid antidepressant effects, occurring a few days of treatment initiation or increasing the dose without eliciting any initial psychogenic effects (Zhou et al., 2013).

In conclusion, the present study provided important additional evidence that DM has antidepressant-like actions. Moreover, AMPA receptor stimulation appears to play a pivotal role in facilitating the antidepressant-like effects of DM. Together with earlier studies, these data strongly warrants the need to further explore the translational potential of DM as safe and efficacious rapid acting antidepressant.

Acknowledgments: The technical assistance of Ying Huang Zhang, Anna Scandinaro, Ashley Brandebura, and Maria Voronkova in scoring the TST data is appreciated. This work was supported by a Research Fund Development Grant from West Virginia University. Portions of this chapter were published in part or in full as: Nguyen, L., and Matsumoto, R.R. (2015). Involvement of AMPA receptors in the antidepressant-like effects of dextromethorphan in mice. Behav Brain Res. 


\section{CHAPTER 5}

Behavioral and biochemical analyses of effects of DM in relation to its antidepressant-like effects 


\subsection{Introduction}

In Chapters 3 and 4, we have shown that DM exerts antidepressant-like effects in mice using the FST and TST, two well-established and commonly used animal models for predicting antidepressant efficacy (Cryan and Holmes, 2005; McArthur and Borsini, 2006; Nestler et al., 2002b). We have also shown the involvement of sigma-1 and AMPA receptors, two molecular targets associated with producing a faster onset of antidepressant efficacy (Adell et al., 2005; Duman, 2014b; Hayashi and Su, 2008), in mediating the antidepressant-like effects of DM in these behavioral tests (Nguyen and Matsumoto, 2015; Nguyen et al., 2014). These results lend credence to DM as a potential antidepressant warranting further investigation.

An important study that remains to be pursued is the potential effects of DM on antidepressantrelevant neural adaptations. A growing body of evidence suggests that neural plasticity may be a final common pathway of different antidepressant therapies and may explain the delay in efficacy with conventional antidepressants (Duman, 2014a; Krishnan and Nestler, 2008). An important player thought to be involved in driving the antidepressant-relevant neural adaptations is BDNF (Castren and Rantamaki, 2010). BDNF is formed from its precursor form, pro-BDNF, and both pro-BDNF and mature BDNF levels have been shown to be altered in animal models of depression and in humans with this mood disorder (Castren and Rantamaki, 2010). Interestingly, chronic but not acute treatment with classic antidepressant drugs induces increases in BDNF expression in vivo (Castren and Rantamaki, 2010), whereas acute or chronic treatment with ketamine can promote increases in BDNF (and pro-BDNF) expression (Autry et al., 2011; Garcia et al., 2008; Reus et al., 2011; Reus et al., 2014; Yang et al., 2013; Zhou et al., 2014). Thus, pharmaceutical agents capable of quickly enhancing BDNF (and possibly proBDNF) levels may aid in the development of novel, effective, and faster acting antidepressant drugs.

In the present study, using previously established antidepressant effect doses by our lab, the behavioral effects of DM were re-established and additional neurobiochemical analyses were undertaken. Specifically, the effects of DM on antidepressant-like effects in the FST, the most predictive rodent model for screening potential antidepressant drugs (Cryan and Holmes, 2005; McArthur and Borsini, 2006; 
Nestler et al., 2002b), and locomotor activity in the OFT were studied in mice. In addition, the effects of $\mathrm{DM}$ on pro-BDNF and BDNF protein expression in the hippocampus and frontal cortex, two key areas involved in depression (Palazidou, 2012), were evaluated via Western blot in relation to its antidepressant-like effects. Because the precursor and mature forms of BDNF may be independently regulated and have been separately shown to rapidly increase following ketamine administration (Autry et al., 2011; Garcia et al., 2008; Reus et al., 2011; Reus et al., 2014; Yang et al., 2013; Zhou et al., 2014), we evaluated them individually rather than as a ratio or altogether as just BDNF. Also, because the antidepressant-like behaviors of DM have only been established recently and at a single time point (Nguyen and Matsumoto, 2015; Nguyen et al., 2014), we used this known antidepressant effective time point to evaluate potential biochemical changes. Fast acting and conventional antidepressants represented by ketamine and imipramine, respectively, were used as reference ligands in these assays.

\subsection{Results}

5.2.1. DM produces antidepressant-like effects without changes in locomotor activity

Using previously established antidepressant effective doses of the compounds of interest (Nguyen and Matsumoto, 2015; Nguyen et al., 2014), we confirmed that the fast acting antidepressant drug ketamine and conventional antidepressant imipramine both reduced immobility time in the FST (Figure 12A B; $\mathrm{t}=4.31, \mathrm{P}<0.001 ;$ and $\mathrm{t}=6.22, \mathrm{P}<0.0001$, respectively). Similar to the reference ligands, $\mathrm{DM}$ significantly reduced immobility time (Figure $12 \mathrm{C} ; \mathrm{t}=5.00, \mathrm{P}<0.0001$ ).

In the OFT, ketamine produced significant increases in locomotor activity (Figure 13D; $t=4.14$, $\mathrm{P}<0.001$ ). Imipramine and DM, on the other hand, did not significantly alter locomotor activity (Figure $13 \mathrm{E}$ and $\mathrm{F} ; \mathrm{t}=0.48$ and $\mathrm{t}=0.67$, n.s., respectively).

5.2.2. DM does not alter pro-BDNF or BDNF protein expression in the hippocampus or frontal cortex As shown in Figure 14A and B, ketamine significantly increased pro-BDNF ( 32-34 kDa) levels in the hippocampus $(\mathrm{t}=2.53, \mathrm{P}<0.05)$, but not the frontal cortex $(1.64$, n.s. $)$ approximately 40 min post- 
injection. Imipramine did not alter pro-BDNF protein expression in the hippocampus $(\mathrm{t}=0.26$, n.s.) or frontal cortex ( $\mathrm{t}=1.37$, n.s.). Similar to imipramine, DM also did not increase pro-BDNF levels in the hippocampus ( $t=0.71$, n.s.) or frontal cortex ( $t=0.83$, n.s.).

With regards to the mature form of BDNF ( 18 kDa), no alterations in BDNF protein expression were observed in the hippocampus (Figure 15A) or frontal cortex (Figure 15B) for any of the drugs tested (ketamine: $\mathrm{t}=0.13$ or 0.03 ; imipramine: $\mathrm{t}=0.78$ or 0.95 ; DM: $\mathrm{t}=1.55$ or 0.37 , respectively).

5.2.3. Ketamine produces antidepressant-like effects in the FST, stimulatory effects in the OFT, and increases in hippocampal pro-BDNF protein expression in an AMPA-receptor dependent manner

AMPA receptors have been shown to mediate many of the antidepressant-relevant behavioral and biochemical effects of ketamine (Nguyen and Matsumoto, 2015; Autry et al., 2011; Koike et al., 2011; Maeng et al., 2008). Pretreatment with an AMPA receptor antagonist (NBQX) attenuated the antidepressant-like effects of ketamine in the FST (Figure 16A), stimulatory effects in the OFT (Figure 16B), and increase in pro-BDNF protein expression in the hippocampus (Figure 16C). For the FST antagonism study, the overall ANOVA was significant $(\mathrm{F}[2,27]=6.85 ; \mathrm{P}<0.01)$. Post-hoc Tukey's multiple comparisons tests confirmed that the ketamine treatment group differed significantly from saline $(\mathrm{q}=5.20, \mathrm{P}<0.01)$, and the NBQX + Ketamine group did not differ significantly from saline $(\mathrm{q}=2.09$, n.s. $)$. However, the differences in the effects of ketamine in the absence and presence of NBQX were not quite statistically significant ( $q=3.11$, n.s.), reflecting partial attenuation of the effects. In the OFT, the overall ANOVA was significant $(\mathrm{F}[2,27]=17.73 ; \mathrm{P}<0.0001)$. Post-hoc Tukey's test confirmed the ability of NBQX to significantly block the stimulatory effects of ketamine $(\mathrm{q}=7.55, \mathrm{P}<0.001)$. For hippocampal pro-BDNF levels, the overall ANOVA was also significant $(\mathrm{F}[2,23]=4.78, \mathrm{P}<0.05)$. Post-hoc Tukey's multiple comparisons tests revealed that the ketamine treatment group differed significantly from saline $(\mathrm{q}=3.96, \mathrm{P}<0.05)$, as well as the NBQX + Ketamine group $(\mathrm{q}=3.68, \mathrm{P}<0.05)$. Although not shown, the NBQX treatment group had no significant effects in the FST ( $\mathrm{t}=0.19$, n.s.), OFT $(\mathrm{t}=1.02$, n.s.) or on hippocampal pro-BDNF protein expression ( $\mathrm{t}=1.86$, n.s.) compared to the saline control group. 
Figure 12. Antidepressant effects of ketamine, imipramine, and DM in the FST using previously established antidepressant effective doses. Data shown are expressed as mean \pm SEM. $* * * \mathrm{P}<0.001, * * * * \mathrm{P}<0.0001$ vs. saline-treated group; Student's unpaired t-test. Sal, saline; KET, ketamine; IM, imipramine; DM, dextromethorphan.

A.

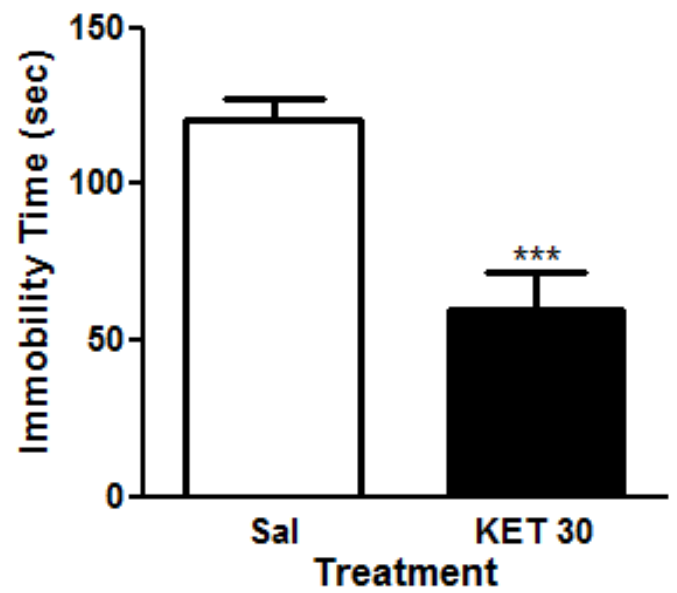

B.

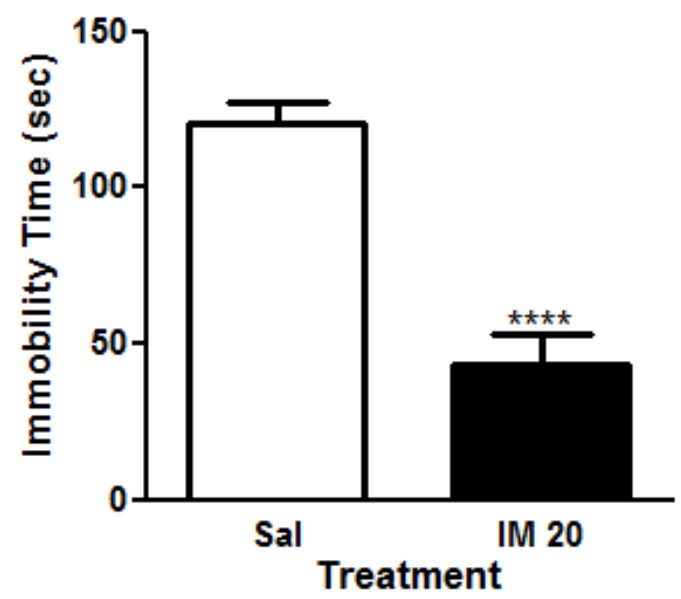

C.

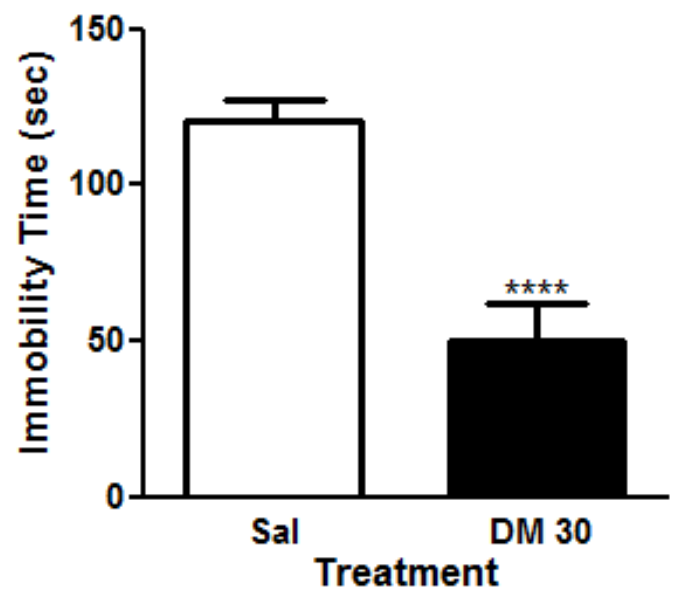

Figure 13. Effects of ketamine, imipramine, and DM in the OFT. Data shown are expressed as mean \pm SEM. $* * * P<0.001$ vs. saline-treated group; Student's unpaired t-test. Sal, saline; KET, ketamine; IM, imipramine; DM, dextromethorphan.
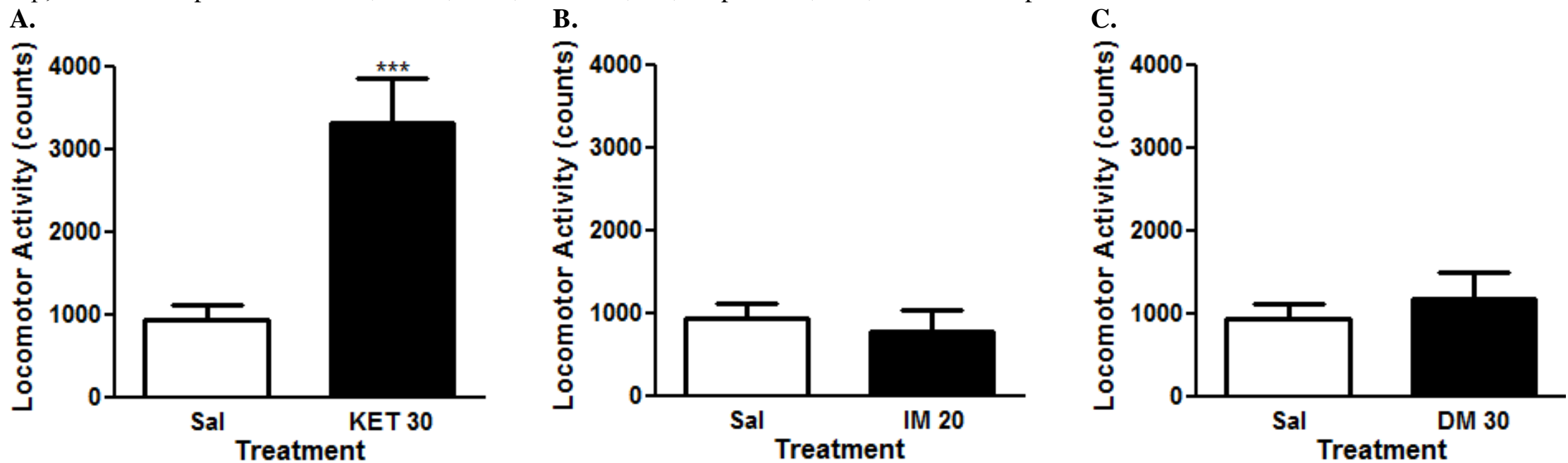
Figure 14. Effects of ketamine, imipramine, and DM on pro-BDNF levels in mouse hippocampus (A) and frontal cortex (B). Representative images of the protein are shown on the left and the cumulative graphed data on the right. Data shown are expressed as mean \pm SEM. *P< 0.05 vs. saline-treated group; Student's unpaired t-test. Sal, saline; KET, ketamine; IM, imipramine; DM, dextromethorphan.

A.
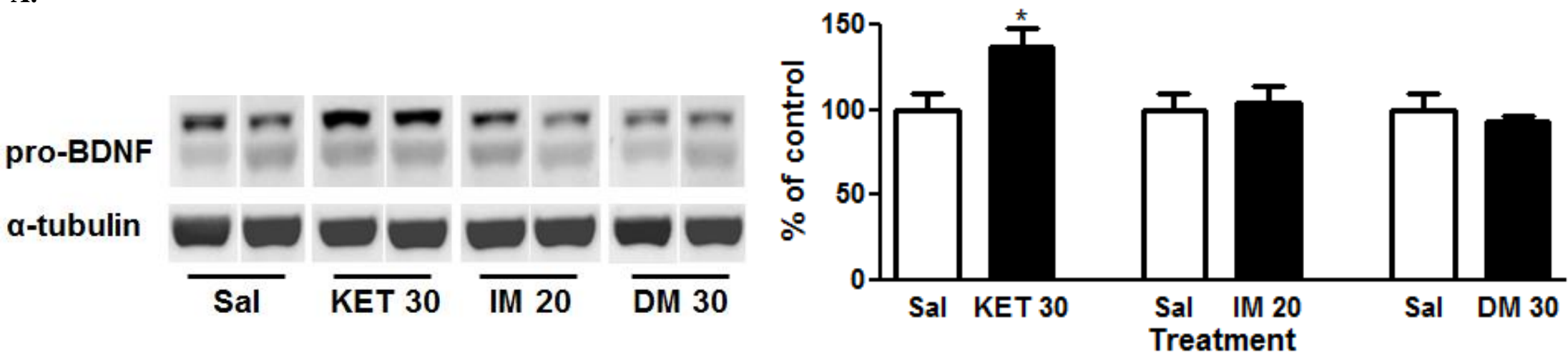

B.
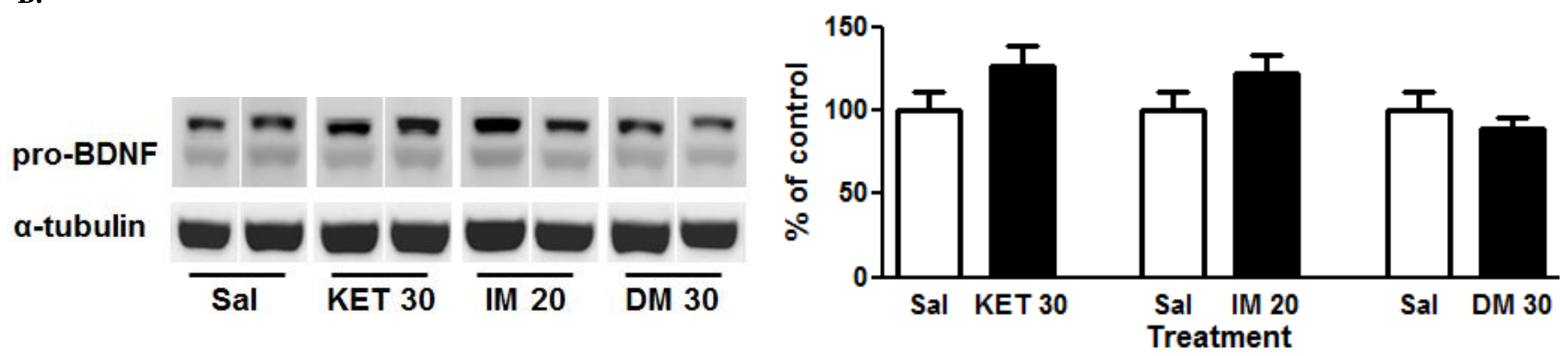
Figure 15. Effects of ketamine, imipramine, and DM on BDNF levels in hippocampus (A) and frontal cortex (B). Representative images of the protein are shown on the left and the cumulative graphed data on the right. Data shown are expressed as mean \pm SEM. Sal, saline; KET, ketamine; IM, imipramine; DM, dextromethorphan

A.
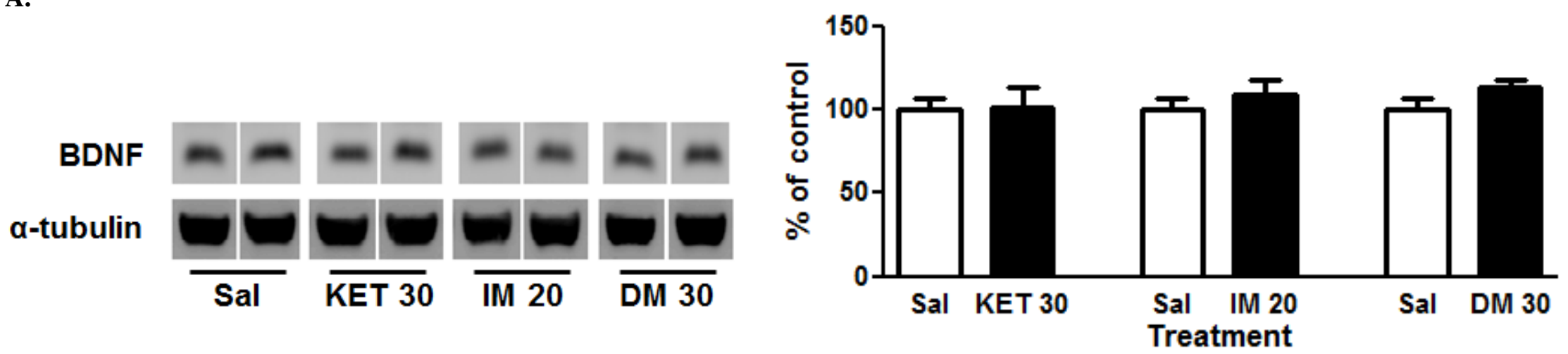

B.
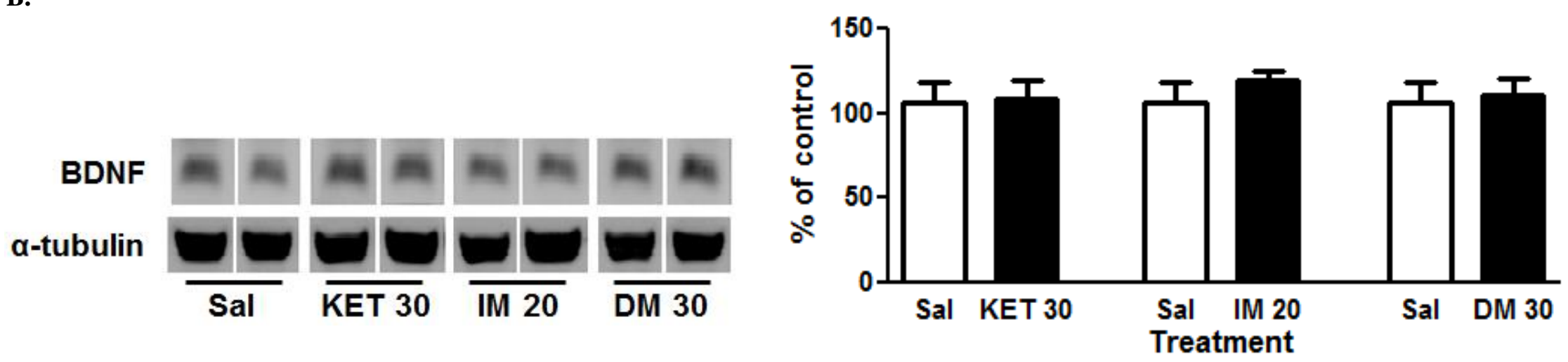
Figure 16. Blockade of AMPA receptors prevented the ketamine-induced behaviors in the FST (A) and OFT (B), and increase in hippocampal pro-BDNF levels (C). Representative images of the protein are shown of the left and the cumulative graphed data on the right (C). Data shown are expressed as mean \pm SEM. $* \mathrm{P}<0.05$, $* * \mathrm{P}<0.01$, $* * * \mathrm{P}<0.001$, vs. saline-treated group; $\# \mathrm{P}<0.05$, \#\#\#P<0.001, vs. ketamine-treated group; one-way ANOVA, followed by post-hoc Tukey's tests. Sal, saline; KET, ketamine.

A.

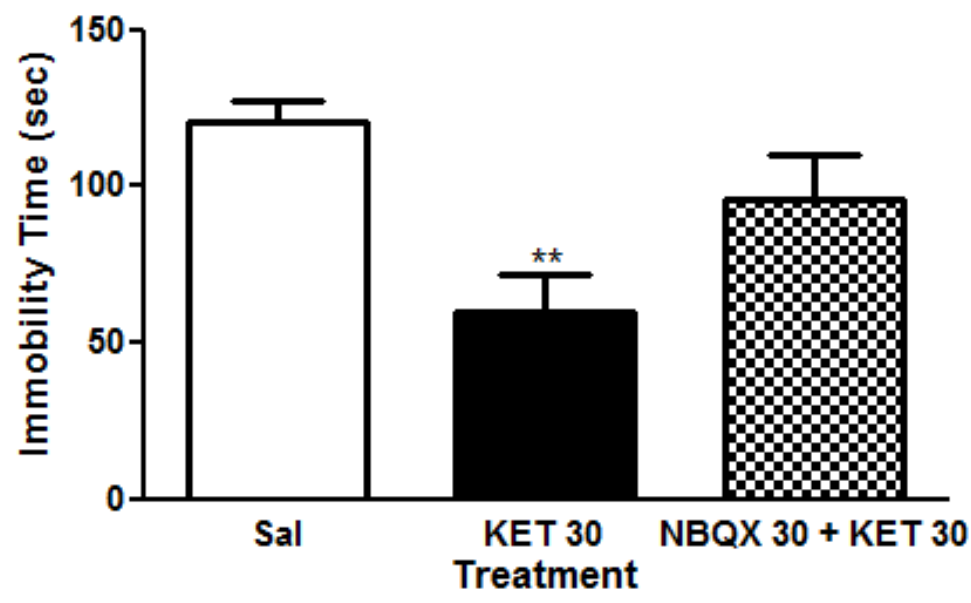

C.

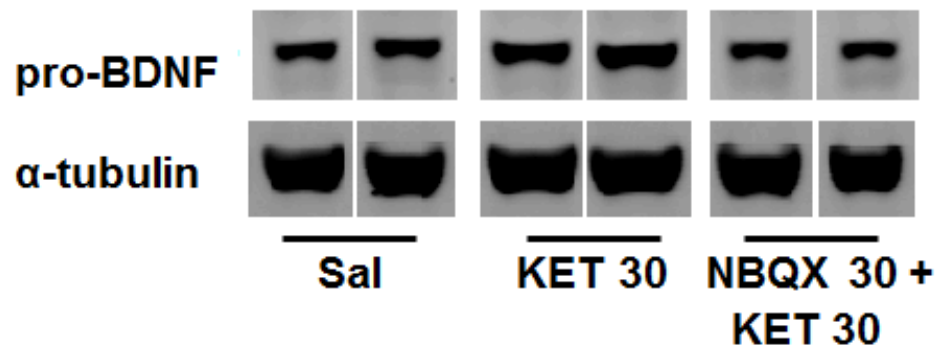

B.
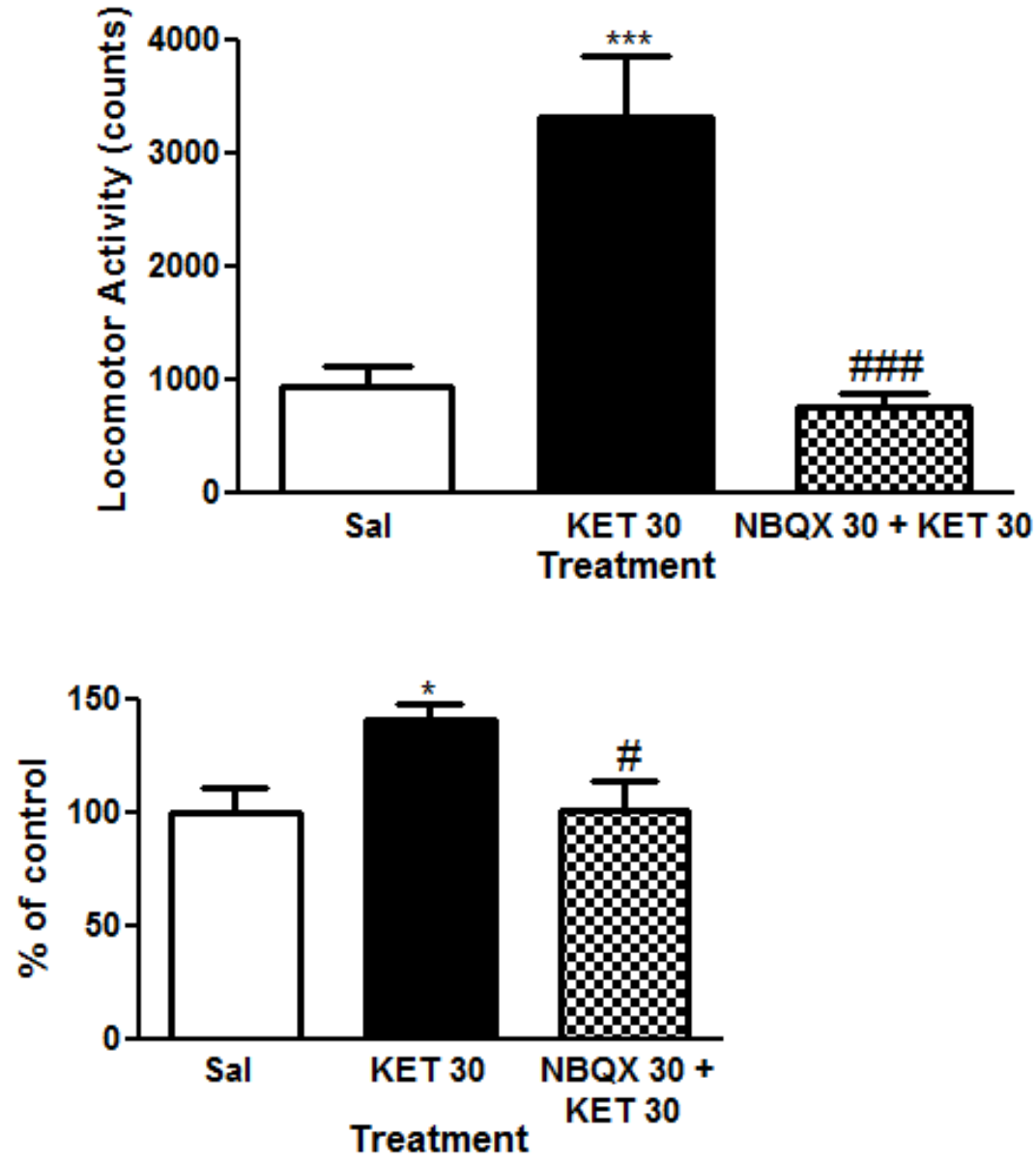


\subsection{Discussion}

Our results indicate that DM reduced immobility time in the FST in mice comparable to the fast acting antidepressant ketamine and TCA imipramine. This is consistent with previous findings from our lab, which showed dose-dependent decreases in the immobility time of mice following administration of DM, ketamine or imipramine (Nguyen and Matsumoto, 2015; Nguyen et al., 2014; Robson et al., 2012).

Moreover, we demonstrated that DM did not affect spontaneous locomotor activity in the OFT, in contrast to ketamine which elicited significant stimulant effects. This is also in line with one of our previous studies (Nguyen and Matsumoto, 2015). The co-incidence of stimulatory effects with antidepressant-like actions for ketamine suggests that the two effects can be related, though not necessarily dependent on one another. In depressed patients treated with ketamine, significant relationships between ketamine's rapid antidepressant and acute psychotomimetic effects have also been observed (Luckenbaugh et al., 2014; Sos et al., 2013). These CNS effects are likely mediated by activation of common mediators (for example, AMPA receptors, as shown in Chapter 4) of both antidepressant and psychostimulant responses vs. producing antidepressant actions secondary to its ability to produce psychostimulant effects. It is important to note that ketamine has been shown to produce rapid clinical antidepressant effects without inducing psychotomimetic effects in some human studies (Lapidus et al., 2014; Valentine et al., 2011), and a slightly higher dose of ketamine (40 mg/kg, i.p.) than used here has been shown to exhibit antidepressant-like effects in the FST without affecting locomotor activity in the OFT under the same experimental conditions (Robson et al., 2012). Regardless, the fact that ketamine had significant stimulatory effects and DM did not further supports that DM may elicit antidepressant efficacy with less abuse liability and untoward side effects.

Next, this is the first study to report a rapid increase in pro-BDNF in the mouse hippocampus following a single administration of ketamine, but not imipramine or DM. The co-incidence of the decrease of immobility time and the increase of pro-BDNF after ketamine, but not imipramine treatment may in part explain ketamine's rapid compared to imipramine's delayed onset of antidepressant efficacy in human populations. We observed an increase in pro-BDNF within $40 \mathrm{~min}$ of i.p. injection in the mice, 
which is a similar time frame to the detection of antidepressant effects reported for i.v. or intranasal ketamine administration in some depressed subjects (DiazGranados et al., 2010b; Lapidus et al., 2014; Luckenbaugh et al., 2014; Zarate et al., 2012).

Many studies have implicated the important role of BDNF in depression (Castren and Rantamaki, 2010) and have examined the effects of ketamine on BDNF levels, but not specifically pro-BDNF (Autry et al., 2011; Garcia et al., 2008; Reus et al., 2011; Yang et al., 2013; Zhou et al., 2014). Indeed, acute administration of ketamine has been shown to increase BDNF levels in rodents in key brain regions involved in depression (Autry et al., 2011; Garcia et al., 2008; Reus et al., 2011; Yang et al., 2013; Zhou et al., 2014). Following a single i.p. administration with ketamine, increases in BDNF levels have occurred as early as $30 \mathrm{~min}$ post-injection in the mouse and rat hippocampus (Autry et al., 2011; Yang et al., 2013). We, however, observed no changes in BDNF levels at 40 min post-injection, which, compared to the earlier mouse study, may be due to differences in mouse strains (Swiss Webster vs. C57BL/6), ketamine doses used (30 mg/kg, i.p. vs. $3 \mathrm{mg} / \mathrm{kg}$, i.p.), and experimental laboratory conditions (tissue analyzed following drug treatment and behavioral testing vs. following just drug treatment). It is important to note that similar to our finding, some other studies, using different strains and species of rodents and doses of ketamine, have also reported a dissociation between antidepressant-like effects and mature BDNF protein changes following ketamine administration (Garcia et al., 2009; Lindholm et al., 2012), wherein stressed and non-stressed male Wistar rats were treated with $15 \mathrm{mg} / \mathrm{kg}$, i.p. in one study (Garcia et al., 2009) and male C57BL/6 mice were treated with $50 \mathrm{mg} / \mathrm{kg}$, i.p. in another study (Lindholm et al., 2012). The possibility that other (non-BDNF) signaling pathways may also mediate ketamineinduced antidepressant effect is supported by studies in BDNF knockout mice. In particular, treatment with ketamine $(50 \mathrm{mg} / \mathrm{kg}$, i.p.) retained antidepressant-like effects in the FST in heterozygous BDNF knockout $(b d n f+/-)$ C57BL/6 mice and failed to alter BDNF levels in the hippocampus when assessed at 45 min or 7 days after drug administration (Lindholm et al., 2012), suggesting ketamine can produce antidepressant-like effects independent of the mature BDNF signaling pathway. 
The incongruity between an increase in pro-BDNF levels and no corresponding change in BDNF levels in our study remains unclear. BDNF is synthesized as a glycosylation precursor protein, proBDNF. It was initially thought that only secreted mature BDNF was biologically active, and that proBDNF, which localizes within the cell, served as an inactive precursor. However, emerging evidence indicate that both pro-BDNF and mature BDNF can be secreted and elicit different and seemingly opposing biological effects via the p75 neurotrophin receptor $\left(\mathrm{p} 75^{\mathrm{NTR}}\right)$ and $\mathrm{TrkB}$, respectively (Castren and Rantamaki, 2010; Lu et al., 2005). Activation of $\mathrm{p} 75^{\mathrm{NTR}}$ by pro-BDNF has been shown to induce neuronal atrophy and apoptosis, whereas activation of the TrkB has been associated with growth and survival, suggesting a "yin and yang" model of neurotrophin action (Lu et al., 2005). Which one of these activities predominates at given times is not yet clear, and whether the rise in pro-BDNF levels following acute ketamine administration in this study is detrimental at the cellular level will need further investigation. It may be that the dynamic changes in pro-BDNF and BDNF levels are important for the optimal tuning of neuronal plasticity, whereby structural increases are balanced by programmed neuronal death, neurite retraction and synaptic pruning (Castren and Rantamaki, 2010; Lu et al., 2005). Indeed, recent findings suggest pro-BDNF can regulate hippocampal long-term depression (LTD) whereas mature BDNF regulates LTP (Lu et al., 2005), two cellular mechanisms which commonly affect the efficacy of a synapse (Bliss and Cooke, 2011).

Lastly, we also report herein that the increase in pro-BDNF levels in the hippocampus and antidepressant-like effects are mediated in part through AMPA receptors. In a study by Reus and colleagues, specific examination of the effects of ketamine on pro-BDNF in rats revealed that ketamine increased this protein expression in the hippocampus and nucleus accumbens, with an MAPK (mitogenactivated protein kinase) kinase (MEK) inhibitor blocking the effects of ketamine in the nucleus accumbens (Reus et al., 2014). They also reported that the MEK inhibitor attenuated the antidepressantlike effects of ketamine in the FST. Our findings thus provide an additional (or upstream) mechanism by which ketamine may be promoting pro-BDNF levels in specific brain regions and are consistent with 
previous reports implicating the critical role of AMPA receptors in mediating the antidepressant-like actions of ketamine (Autry et al., 2011; Koike et al., 2011; Maeng et al., 2008).

Importantly, our data did not evaluate the effects of ketamine or DM on pro-BDNF or BNDF levels at other time points or on other trophic factors. Of note, in a double-blind study involving bipolar patients (type I or II) given placebo or DM (30 or $60 \mathrm{mg} /$ day) in combination with VPA for 12 weeks, participants had lower levels of plasma BDNF compared to healthy controls at baseline (Chen et al., 2014). Subsequent treatment with DM (60 mg/day) plus VPA produced a small increase in plasma BDNF levels from baseline at 12 weeks, and the increase was significantly higher than placebo plus VPA group (Chen et al., 2014). There was trend toward an increase in BDNF found in the DM group. Although it did not correlate with measured improvements of clinical symptoms in this study, the small increase may have benefit on other factors of clinical presentation not measured, such as a shorter duration of clinical course or on other depressive conditions (Chen et al., 2014). Several studies have shown that serum BDNF levels are reduced in unipolar and bipolar depression and can be normalized by successful treatment (Castren and Rantamaki, 2010; Grande et al., 2010); though it remains unclear whether serum BDNF levels might reflect or contribute to the BDNF levels in the brain, as BDNF does not readily cross the blood-brain barrier (Castren and Rantamaki, 2010). Also, in a retrospective study, daily administration of DM/quinidine has been shown to produce antidepressant effects in some bipolar depressed patients within 1-2 days of treatment (Kelly and Lieberman, 2014), rather than hours as seen with ketamine. Further studies aiming to determine whether DM can promote increases in BDNF or pro-BDNF at a later time point are thus worthy of doing.

In addition, we have previously shown that DM produces antidepressant-like effects in the FST in part through a sigma-1 receptor dependent mechanism (Nguyen et al., 2014). Noteworthy, several antidepressants (e.g., fluoxetine, fluvoxamine, imipramine, and ketamine) have been shown to enhance NGF activity through a sigma-1 receptor dependent mechanism (Nishimura et al., 2008; Robson et al., 2012; Takebayashi et al., 2002) and NGF itself can produce antidepressant-like effects (Overstreet et al., 2010). DM, through activation of sigma-1 receptors, may promote the activation of other trophic factors 
such as NGF to facilitate neural adaptations. This is an area that together with additional time course studies warrants further investigation.

In conclusion, our results suggest that DM may produce antidepressant actions with a reduction in problematic stimulant effects compared to ketamine. Moreover, in contrast to the AMPA-dependent antidepressant-like effects and increase in pro-BDNF levels of ketamine, DM does not rapidly alter proBDNF levels in the hippocampus and frontal cortex. Together with earlier studies, these findings indicate that DM shares some, but not all antidepressant mechanisms with ketamine. In light of the recent clinical report of DM's efficacy (some within 1-2 days) for bipolar depression and our preclinical findings, additional studies are needed to identify specific molecular mechanisms through which DM conveys its antidepressant effects, as well as to further examine DM's potential as a safe and effective fast acting antidepressant.

Acknowledgments: We appreciate the technical contributions of Brandon Lucke-Wold and Aric Logsdon. This data was presented at the Experimental Biology 2015 annual meeting: Nguyen, L., Logsdon, A., Lucke-Wold, B., and Matsumoto, R. (2015). Behavioral and biochemical analyses of the fast acting antidepressant-like potential of dextromethorphan. ABSTRACT, The FASEB Journal 29:931.2. 


\section{CHAPTER 6}

Potentiation of nerve growth factor-induced neurite outgrowth by DM 


\subsection{Introduction}

As discussed in Chapters 2 and 5, increasing evidence suggests that the action of fast acting and conventional antidepressants likely involves neural plasticity; i.e., antidepressants may exert their therapeutic effects by stimulating adaptive changes in the CNS. Indeed, stress as well as psychoactive drugs, including antidepressants, have been reported to induce not only neurobiochemical alterations but also structural alterations and synaptic remodeling, including sprouting of neurites and changes of dendritic spines (Castren and Rantamaki, 2010; Duman, 2014a; Fishback et al., 2010; Nakamura, 1991; Norrholm and Ouimet, 2001; Pittenger and Duman, 2007). In animal studies, in particular, several classes of antidepressants could reverse the reductions in the density of dendritic spines in hippocampal neurons induced by stress or by depression (Duman, 2014a; Norrholm and Ouimet, 2001; Pittenger and Duman, 2007). Additionally, chronic ETC has been shown to induce axonal sprouting in the rodent hippocampus (Gombos et al., 1999; Lamont et al., 2001).

Previous studies in the rat pheochromocytoma PC12 cell line, which has been widely used as a model for studying neuronal differentiation and neurite outgrowth (Greene and Tischler, 1976), have shown that conventional antidepressants such as imipramine, fluvoxamine, fluoxetine, and escitalopram as well as atypical and fast acting antidepressants characterized by mirtazapine and ketamine, respectively, can potentiate NGF-induced neurite outgrowth (Ishima et al., 2014; Nishimura et al., 2008; Robson et al., 2012; Takebayashi et al., 2002). This type of neuronal plasticity may contribute to their therapeutic effects in human populations. In addition, all the mentioned drugs (with the exception of mirtazapine) have moderate to high binding affinity to sigma-1 receptors (Fishback et al., 2010; Ishima et al., 2014; Robson et al., 2012) and have been shown to potentiate NGF-induced neurite outgrowth through activation of sigma-1 receptors (Ishima et al., 2014; Nishimura et al., 2008; Robson et al., 2012; Takebayashi et al., 2002). Selective sigma-1 agonists have also been shown to potentiate NGF-induced neurite outgrowth (Nishimura et al., 2008; Takebayashi et al., 2002), further suggesting a role for sigma-1 receptors in neuronal plasticity. 
DM has high binding affinity to sigma-1 receptors (see Table 1, page 4), and we have shown that it acts as an agonist at sigma-1 receptors to elicit antidepressant-like effects in vivo (Nguyen et al., 2014). However, the ability of DM to modulate antidepressant-relevant neural adaptations has yet to be systematically investigated. The PC12 cell model provides a rational format for the initial evaluation of the potential of DM to promote neuronal remodeling as well as characterization of the specific cellular mechanisms that may be involved. We know that ketamine can promote sigma-1 receptor-mediated neurite outgrowth in PC12 cells (Robson et al., 2012) and hypothesize that DM can do the same. In addition to examining the effects of our drugs of interest in the absence and presence of a sigma-1 antagonist (NE-100, 4-methoxy-3-(2-phenylethoxy)-N,N-dipropylbenzeneethanamine), an AMPA antagonist (NBQX) and specific inhibitors of signaling molecules thought to be downstream players of activation of AMPA receptors [LY294002, 2-(4-morpholinyl)-8-phenyl-1(4H)-benzopyran-4-one, for phosphatidylinositol 3-kinase (PI3K); PD98059, 2-(2-amino-3-methoxyphenyl)-4H-1-benzopyran-4-one, for MAPK; and rapamycin for mammalian target of rapamycin (mTOR)] or trkA receptor (NGF receptor) [same inhibitors for AMPA pathways; and U73122, -[6-[[(17ß)-3-methoxyestra-1,3,5(10)-trien-17yl]amino]hexyl]-1H-pyrrole-2,5-dione, for phospholipase C-gamma (PLC- $\gamma$ )] will be used to begin elucidating the underlying mechanisms and signaling pathways (Figure 17). In this chapter, we show the results of the first part, i.e., whether DM can potentiate NGF-induced neurite outgrowth in PC12 cells. Unfortunately, the evaluation of the hypothesized cellular mechanisms was not pursued in this dissertation due to technical limitations of our current model system. Thus, of great interest for future investigations would be identification of the precise cellular mechanisms underlying the enhancement of DM on neurite outgrowth in vitro and confirmation in vivo.

\subsection{Results}

Examples of cells with neurite outgrowth are shown in Figure 18, with the extension of branching varicose processes similar to those produced by sympathetic neurons in primary cell culture (Bradshaw et al., 1974; Tischler and Greene, 1975). Over the course of 2 days, 0.039 to $10 \mathrm{ng} / \mathrm{mL}$ NGF dose 
dependently induced neurite sprouting in PC12 cells (Figure 19; F[9,44]=15.92, $\mathrm{P}<0.0001$ ). The induction of neurite outgrowth appears to plateau at a final concentration of $2.5 \mathrm{ng} / \mathrm{mL}$ of NGF $(12.61 \pm 1.87 \%)$. Therefore, for subsequent experiments, a final concentration of $0.3125 \mathrm{ng} / \mathrm{mL}(7.16 \pm 0.58 \%)$ was used, as this concentration allowed for bidirectional changes in neurite outgrowth following concomitant exposure to compounds of interest (i.e., low and high enough to allow for visualization of the potentiation or reduction, respectively, of neurite outgrowth).

When the TCA imipramine was tested for its ability to potentiated NGF-induced neurite outgrowth, a one-way ANOVA showed that the changes were significant (Figure 20; F[7,88]=5.65, $\mathrm{P}<0.001)$. Furthermore, post-hoc Dunnett's test revealed that imipramine potentiated NGF-induced neurite outgrowth at the following concentrations: 10 (q=4.23, $\mathrm{P}<0.001)$ and $100(\mathrm{q}=3.22, \mathrm{P}<0.05) \mathrm{nM}$. Ketamine also significantly potentiated NGF-induced neurite outgrowth (Figure 21; F[7,73]=3.08, $\mathrm{P}<0.01)$. Post-hoc tests revealed that ketamine significantly potentiated NGF-induced neurite outgrowth at the following concentrations: $10(\mathrm{q}=3.12, \mathrm{P}<0.05)$ and $100(\mathrm{q}=3.61, \mathrm{P}<0.01) \mathrm{nM}$.

DM, which had no effect on the neurite outgrowth when administered by itself $(F[7,82]=1.39$, n.s.), also significantly potentiated the neurite sprouting induced by $\operatorname{NGF}(F[7,73]=5.71, P<0.001$ ) (Figure 22A and B, respectively). Post-hoc tests revealed that DM significantly potentiated NGF-induced neurite outgrowth at the following concentrations: $10(q=3.17, \mathrm{P}<0.05), 100(\mathrm{q}=4.04, \mathrm{P}<0.001), 1000(\mathrm{q}=3.26$, $\mathrm{P}<0.05)$, and 10,000 (q=4.24, $\mathrm{P}<0.001) \mathrm{nM}$.

\subsection{Discussion}

In the present study, we found that imipramine and ketamine potentiated NGF-induced neurite outgrowth, similar to previous studies (Robson et al., 2012; Takebayashi et al., 2002). In addition, this is the first report of DM potentiating NGF-induced neurite outgrowth in PC12 cells. Despite these promising preliminary results, however, the PC12 model under the current laboratory settings has been a less than ideal cell model, and results have been difficult to reproduce. The PC12 cells appear highly dependent on the cell passage number, the batch of NGF, and/or other experimental conditions. There was 
Figure 17. Proposed signaling pathways involved in stimulation of neurite outgrowth by ketamine and DM. Ketamine may activate AMPA receptors and subsequent downstream signaling pathways (blue arrows) to eventually induce neurite outgrowth. In addition to or separate from AMPA pathways, ketamine may potentiate the activation of NGF-mediated pathways (green arrows) through its agonist activity at sigma-1 receptors (designated by the diamonds), which may modulate and enhance the activity of the different signaling molecules. With its overlapping pharmacology, DM may activate similar pathways.

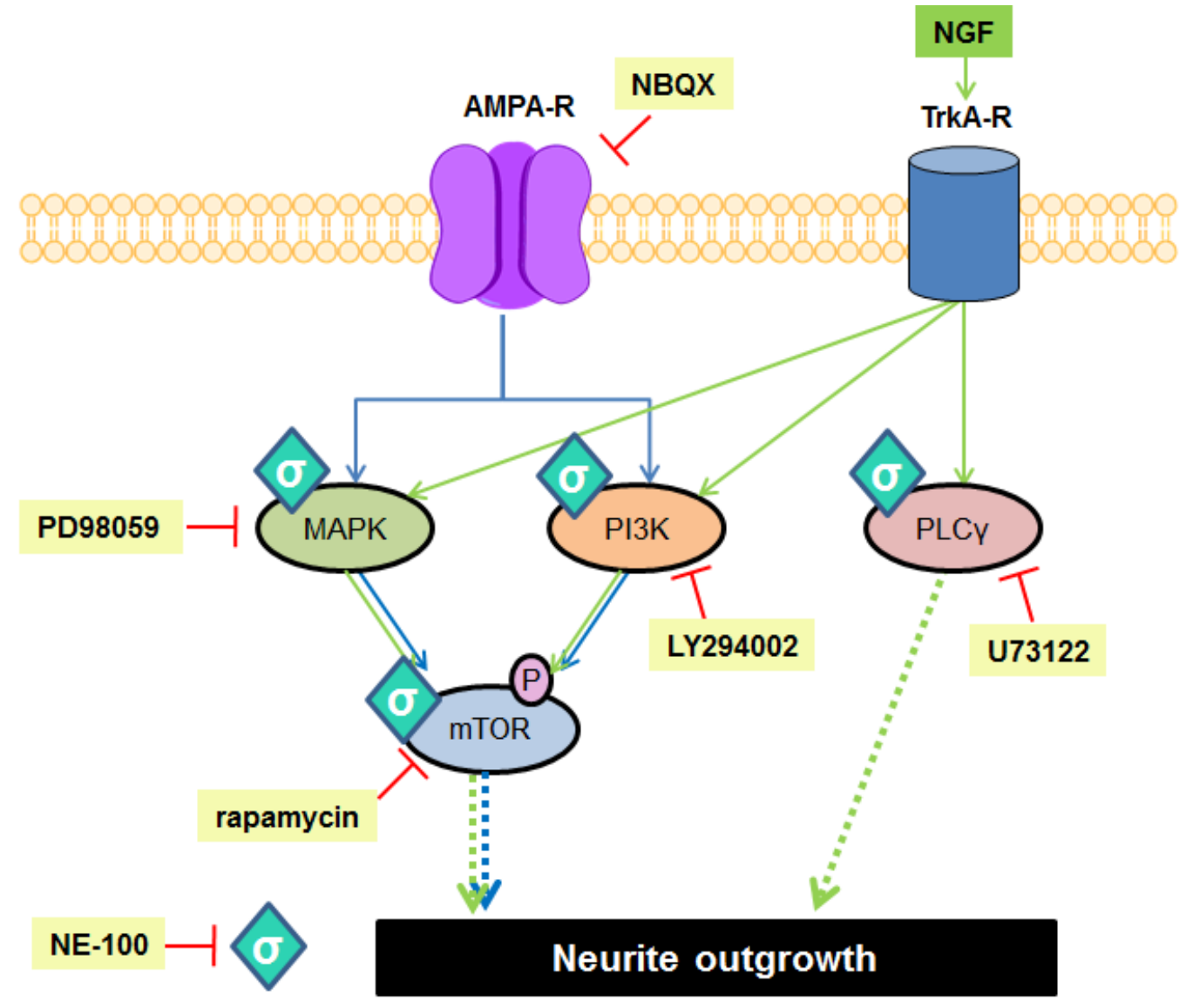


Figure 18. Representative image of neurite sprouting in PC12 cells. Cells were treated with a low concentration $(0.3125 \mathrm{ng} / \mathrm{mL})$ of NGF with concomitant drug exposure (imipramine, $10 \mathrm{nM}$ ) for $48 \mathrm{hr}$. The magnification was 10-fold, with the enlarged picture showing cells with neurites, indicated with an arrowhead.

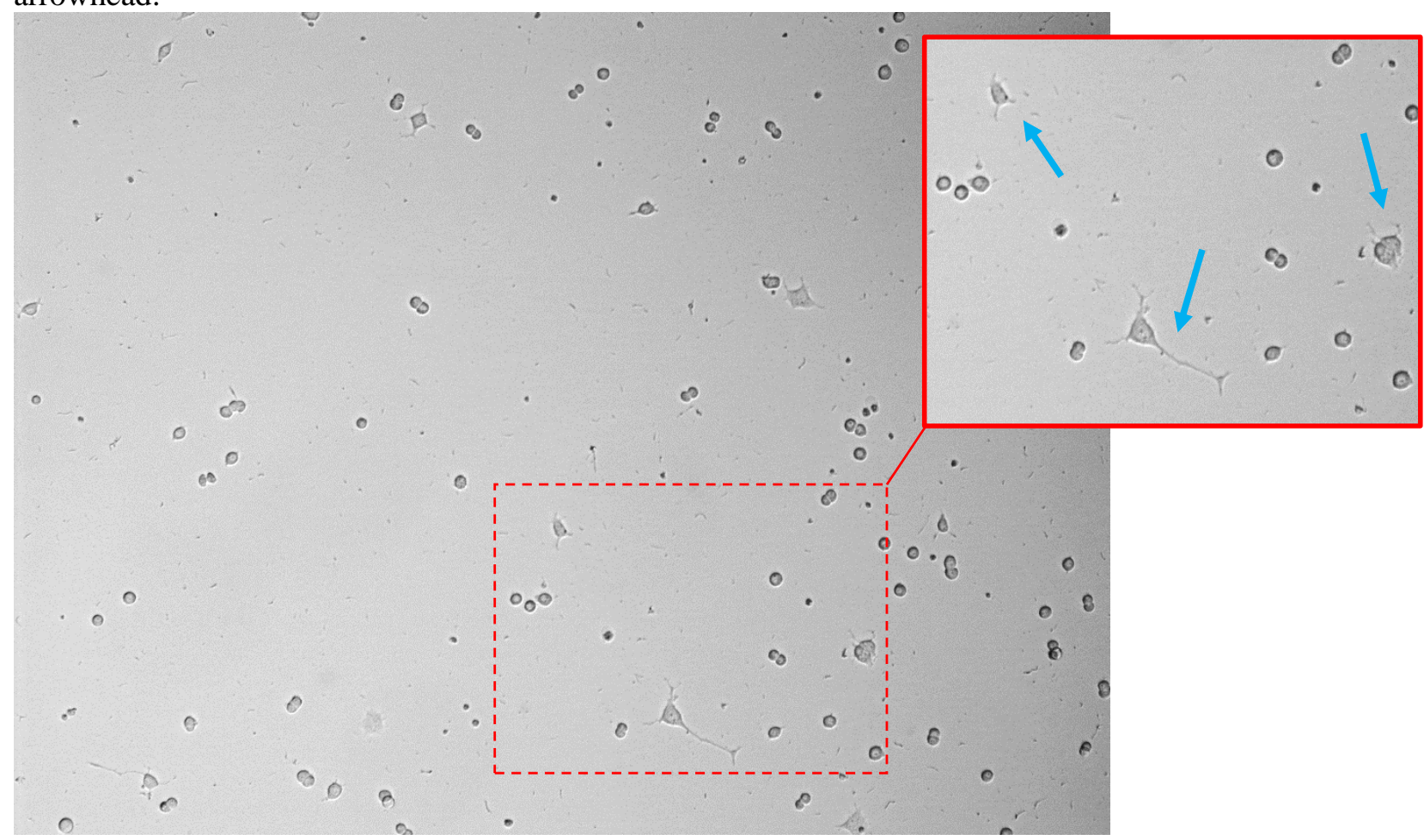

Figure 19. NGF induces neurite outgrowth in PC12 cells. At least two separate experiments were carried out. Data shown are expressed as the mean \pm SEM. $* \mathrm{P}<0.05$, $* * \mathrm{P}<0.01$, $* * * \mathrm{P}<0.001$, vs. vehicle control; one-way ANOVA followed by post-hoc Dunnett's tests.

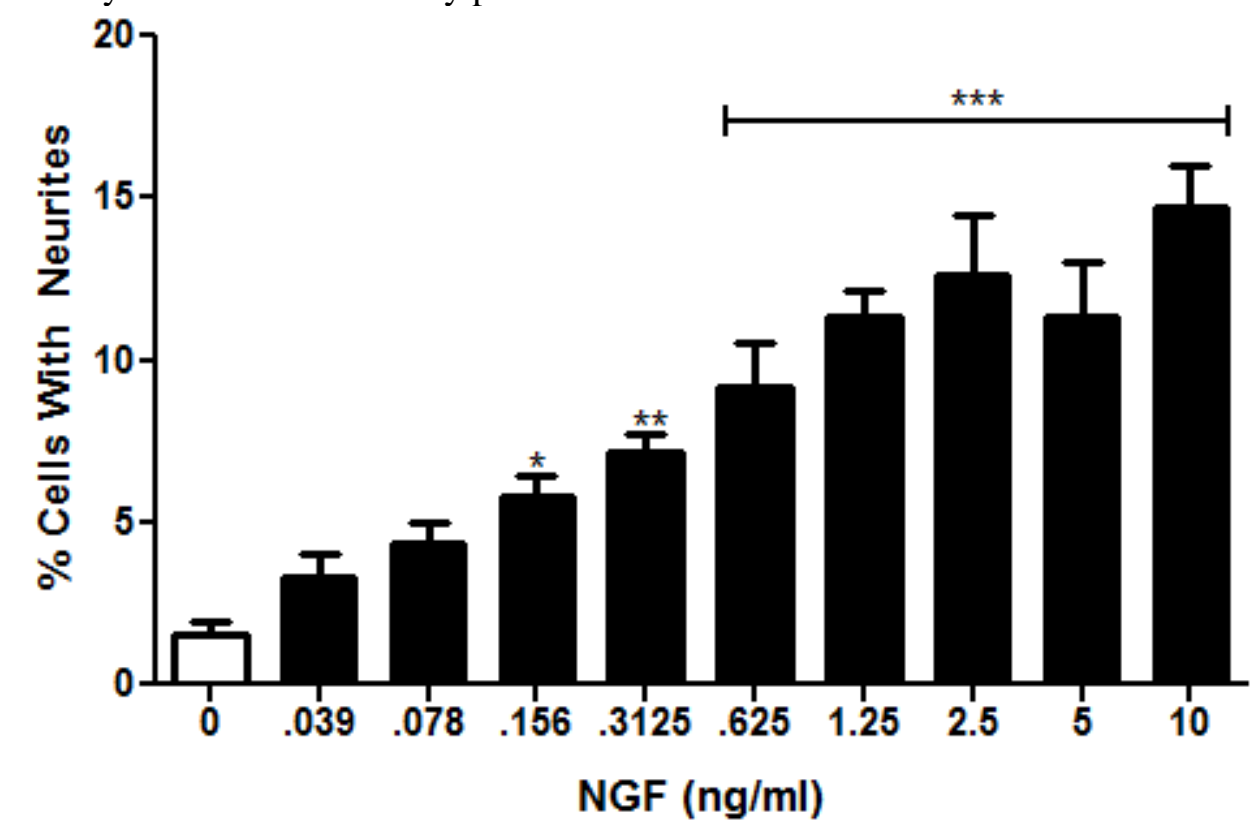


Figure 20. Imipramine potentiates NGF-induced neurite outgrowth in PC12 cells. At least two separate experiments were carried out. Data shown are expressed as the mean $\pm \mathrm{SEM}$. $* \mathrm{P}<0.05$, $* * * \mathrm{P}<0.001$, vs. vehicle control; one-way ANOVA followed by post-hoc Dunnett's tests.

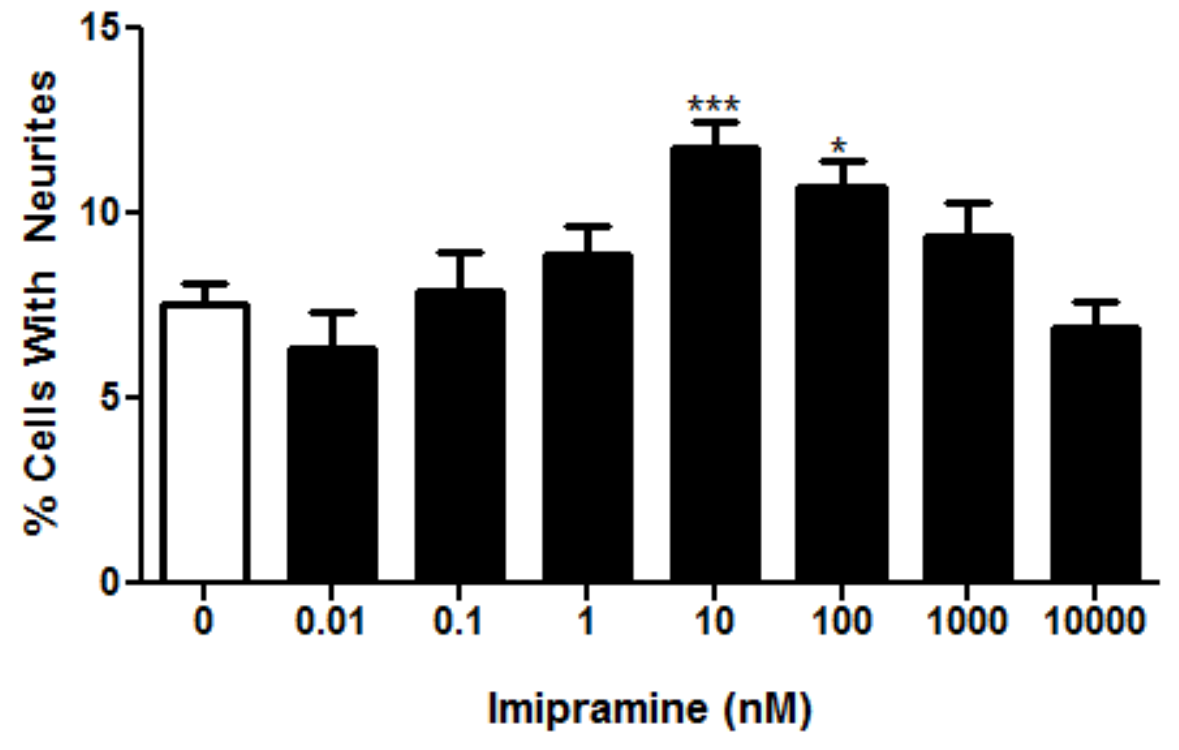

Figure 21. Ketamine potentiates NGF-induced neurite outgrowth in PC12 cells. At least two separate experiments were carried out. Data shown are expressed as the mean $\pm \mathrm{SEM}$. ${ }^{*} \mathrm{P}<0.05$, $* * \mathrm{P}<0.01$, vs. vehicle control; one-way ANOVA followed by post-hoc Dunnett's tests.

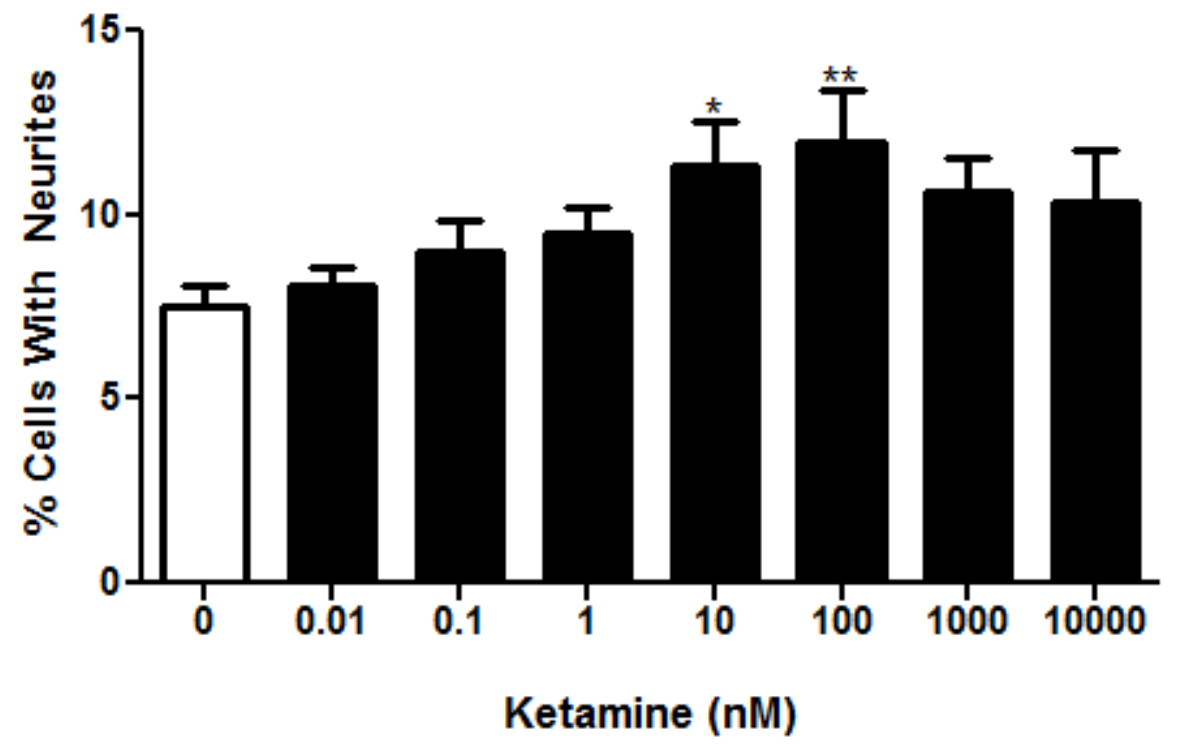


Figure 22. DM does not induce neurite outgrowth alone but potentiates NGF-induced neurite outgrowth in PC12 cells. In the absence of NGF, DM had no effect on neurite sprouting (A). In the presence of NGF, DM significantly potentiated NGF-induced neurite outgrowth in a dose-dependent manner (B). At least two separate experiments were carried out. Data shown are expressed as the mean $\pm \mathrm{SEM} . * \mathrm{P}<0.05$, ***P $<0.001$, vs. vehicle control; one-way ANOVA followed by post-hoc Dunnett's tests.

A.

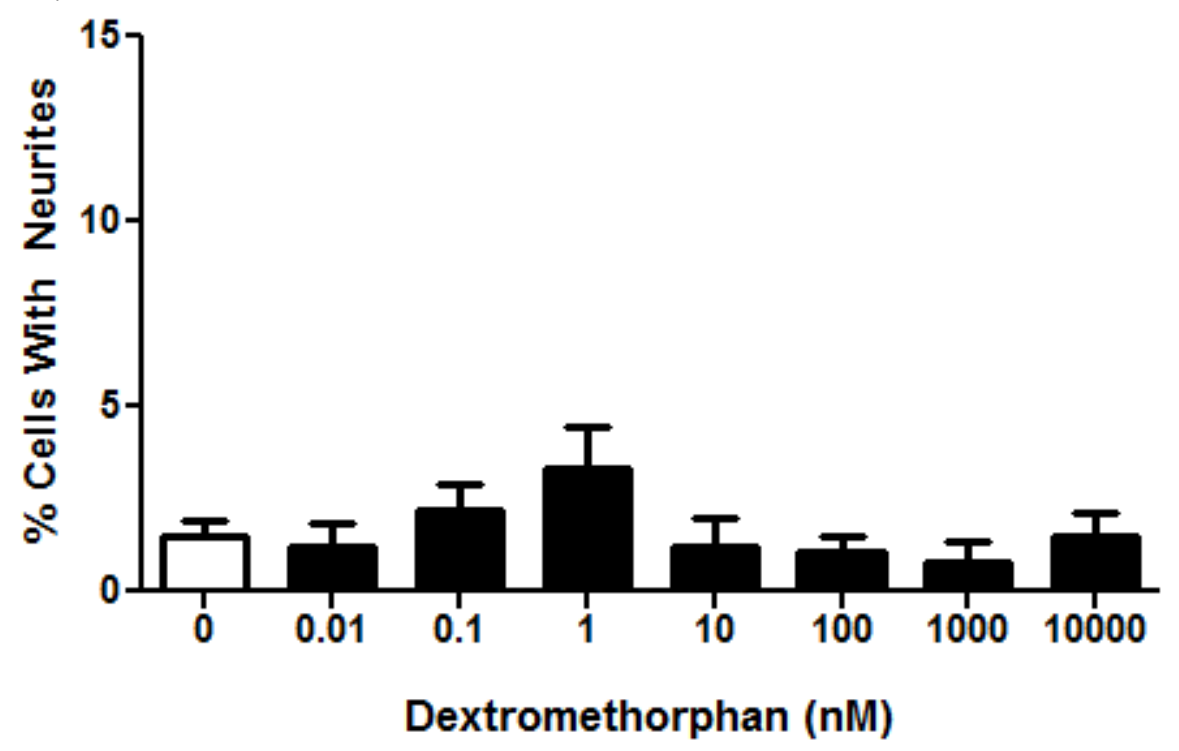

B.

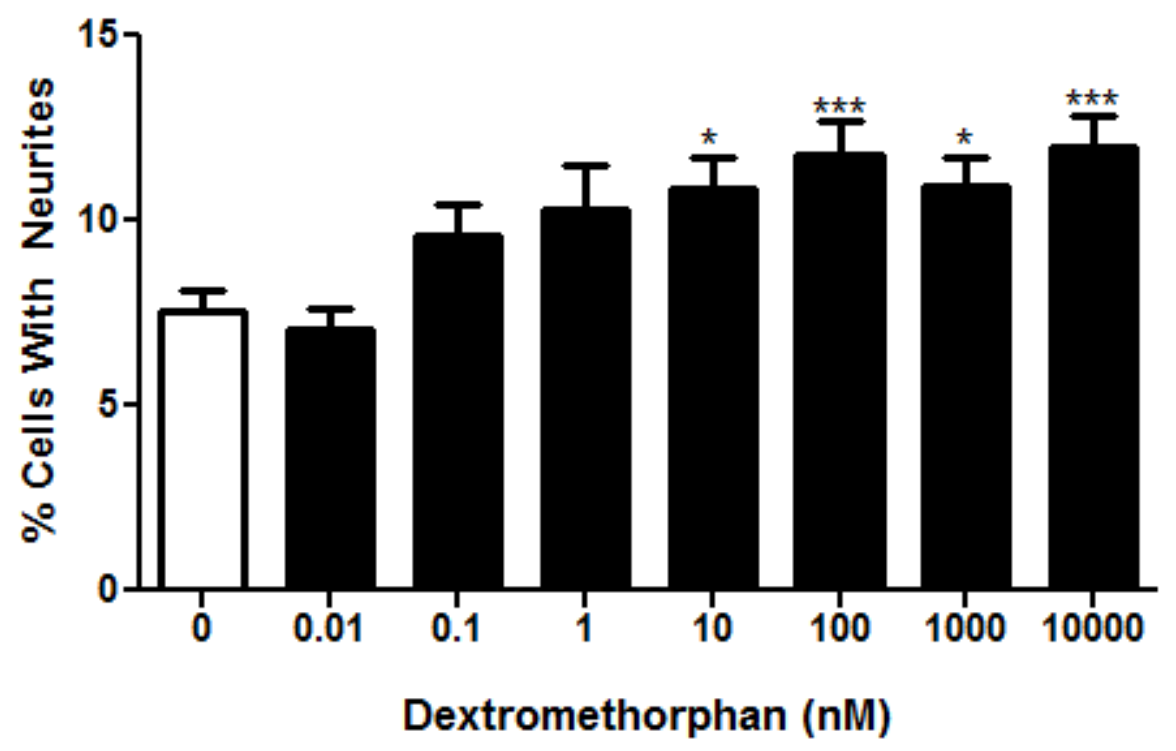


variability in the magnitude of percentage of cells with neurite outgrowth with the different treatments; variability in the drug concentration needed to achieve the maximum percentage of cells with neurite outgrowth; and variability in NGF concentration needed to achieve low percentage (5-8\%) of cells with neurite outgrowth in the vehicle control. These issues have made it difficult to detect reproducible differences between groups in subsequent studies.

In considering whether to pursue additional studies using the PC12 cell model, some advantages and limitations will be mentioned here. One advantage is that the PC12 cells have been widely used as an in vitro model system to study differentiation into a neuronal phenotype (Greene and Tischler, 1982), including studies on the effects of neurotoxicants on differentiation (Slotkin and Seidler, 2009), and neurobiological and neurochemical studies to investigate cytoskeleton behavior (Arregui et al., 1994; Sparatore et al., 2000; Takemura et al., 1993), axonal growth and regeneration (Flynn et al., 2009; Markus et al., 2002; Paveliev et al., 2007), and neurotrophin signaling pathways (Vaudry et al., 2002). PC12 cells originate from adrenal chromaffin cells, whose differentiation into sympathetic neurons can be induced by specific factors including NGF, basic fibroblastic growth factor (bFGF), and cyclic adenosine monophosphate (cAMP) (Sofroniew et al., 2001). Due to their well-characterized response to varying doses of NGF as well as published sigma-mediated effects, PC12 cells provide a good experimental model system for investigating cooperative effects in neurite formation that may be relevant to the effects of DM and other antidepressant drugs. Moreover, the mechanisms of PC12 cell neurite outgrowth are well-documented, which allows for the investigation of specific cellular targets activated by drugs of interest that may be involved to execute a complex process such as neurite extension.

One limitation of PC12 cells is that they have the potential for genetic drift with respect to repeated cell passage with a concomitant change in phenotype (Banker and Goslin, 1998; Harry et al., 1998), which may in part explain our sub-optimal reproducibility; we had used old, frozen aliquots of PC12 cells that had been used in a previous study in the lab (Robson et al., 2012). In addition, although PC12 cells can acquire properties similar to sympathetic neurons, they do not develop definitive axons or 
dendrites (Banker and Goslin, 1998; Greene and Tischler, 1976), and it is unclear if they can form functional synapses between their neurites (Jeon et al., 2010). A potential advantage from this, however, would be that PC12 cells could be used solely for the investigation of neurite outgrowth and primary neuronal cultures, for example, for synaptic plasticity, and that these two distinctive processes can be studied separately. Also, model neuron-like cells (such as PC12) are simpler and more flexible than low density cultures of primary neurons. Alternative studies examining antidepressant-related neural plasticity can focus on hippocampal neurogenesis (Santarelli et al., 2003) and spine formation (Li et al., 2010), both of which have also been shown to partly involve sigma-1 receptors (Matsumoto et al., 2005; Moriguchi et al., 2013; Tsai et al., 2009). 


\section{CHAPTER 7}

Evaluation of the antidepressant-like effects of deuterated DM 


\subsection{Introduction}

Before DM can be tested in depressed patients, one major challenge that must be overcome is its substantial first-pass metabolism. DM is rapidly metabolized primarily by CYP2D6 into its major metabolite DX (Schmid et al., 1985), decreasing its bioavailability and potential antidepressant efficacy. Moreover, increased exposure to DX, a more potent NMDA receptor antagonist than DM itself, increases the risk for PCP-like psychotomimetic effects seen with high dose DM (Miller, 2011).

Two approaches to extend the half-life of DM include 1) adding an inhibitor of CYP2D6, such as quinidine, and 2) chemically modifying DM to inhibit its metabolism. The first strategy has resulted in a combination drug that is approved by the FDA and EMA for the treatment of PBA. The present study focuses on the second strategy, working with a deuterium (d6)-enriched DM. Deuterium is a naturally occurring isotope. It is one of two stable isotopes of hydrogen which contains one proton and one neutron in the nucleus, in contrast to the lighter and more common isotype, protium, which has no neutron (Gant, 2014). Deuterium can make stronger chemical bonds than hydrogen; when this particular structural modification is made to functional groups commonly degraded during metabolism, the modified compound may have enhanced metabolic stability and hence a longer half-life (Gant, 2014).

Using the FST and TST, the two most validated behavioral assays for assessing antidepressant potential (Cryan and Holmes, 2005; Cryan et al., 2005; McArthur and Borsini, 2006; Nestler et al., 2002b), d6-DM was evaluated for antidepressant-like effects. Next, to better characterize the antidepressant-like effects of d6-DM, all subsequent studies were carried out in the FST, the more validated of the two behavioral assays (Cryan and Holmes, 2005; McArthur and Borsini, 2006; Nestler et al., 2002b). As AMPA and sigma-1 receptors have been implicated in mediating the antidepressant-like actions of regular DM (Nguyen and Matsumoto, 2015; Nguyen et al., 2014), an AMPA receptor antagonist (NBQX) and two sigma-1 antagonists (BD1063 and BD1047) were also evaluated in conjunction with d6-DM to determine the involvement of these two receptors in the antidepressant-like effects of d6-DM. Finally, because the deuteration may only partially reduce the formation of metabolites, 
d6-DM was also tested in combination with quinidine to determine if the addition of quinidine may improve its antidepressant-like effects.

\subsection{Results}

\subsubsection{Effects of d6-DM alone in the FST, TST, and OFT}

d6-DM produced a significant dose-dependent decrease in immobility time in the FST and TST (Figure 23A and B; F[3,46]=6.91, $\mathrm{P}<0.001$ and $\mathrm{F}[2,22]=4.99, \mathrm{P}<0.05$, respectively). Post-hoc Dunnett's tests showed that $\mathrm{d} 6-\mathrm{DM}$ at $30 \mathrm{mg} / \mathrm{kg}$ was significantly different from saline $(\mathrm{q}=3.48, \mathrm{P}<0.01$ and $\mathrm{q}=3.04$, $\mathrm{P}<0.05$, in the FST and TST respectively). In the OFT, d6-DM also produced a significant increase in locomotor activity (Figure 23C; F[3,71]=7.33, P<0.001). Post-hoc Dunnett's test confirmed that d6-DM at $30 \mathrm{mg} / \mathrm{kg}$ significantly differed from saline $(\mathrm{q}=6.59, \mathrm{P}<0.001)$. Since $\mathrm{d} 6-\mathrm{DM}$ showed significant stimulant effects, a correlation analysis between locomotor activity and immobility time was carried out to determine whether the stimulant effects could account for its observed antidepressant-like actions. The Pearson's $r$ correlation test including data points from all the doses of d6-DM tested revealed that there was no correlation between the d6-DM-induced alterations in locomotor activity in the OFT and immobility time in the FST and TST (Figure 23D; r=-0.14, n.s.).

\subsubsection{Effects of AMPA or sigma-1 receptor antagonists on d6-DM in the FST and OFT}

Pretreatment with the AMPA receptor antagonist NBQX $(30 \mathrm{mg} / \mathrm{kg})$ failed to prevent the antidepressant-like effects of d6-DM in the FST (Figure 24A). ANOVA confirmed a significant difference between the various treatment groups $(\mathrm{F}[3,41]=10.84, \mathrm{P}<0.001)$. Pairwise comparisons using post-hoc Tukey's multiple comparison tests confirmed that d6-DM significantly reduced immobility time compared to saline $(\mathrm{q}=4.97, \mathrm{P}<0.01)$. However, post-hoc comparisons showed that the NBQX $+\mathrm{d} 6-\mathrm{DM}$ group did not differ significantly from d6-DM alone ( $q=1.24$, n.s.), and had a significant reduction in immobility time compared to saline $(\mathrm{q}=5.21, \mathrm{P}<0.01)$. Pretreatment with the sigma-1 receptor antagonist BD1063 (10 mg/kg) or BD1047 (20 mg/kg) showed a noticeable trend toward the attenuation of the 
Figure 23. Antidepressant-like and stimulatory effects of d6-DM in the FST, TST and OFT. d6-DM produced a significant decrease in immobility time the FST (A) and FST (B) and a significant increase in locomotor activity in the OFT (C). However, no signific ant correlation was observed between the d6-DM-induced alterations in locomotor activity and immobility time (D). Bar graph data shown are expressed as mean \pm SEM. For the correlation graph, black circles represent data points from mice subjected to the FST and blue circles from mice subjected to the TST. $* \mathrm{P}<0.05, * * \mathrm{P}<0.01,{ }^{*} * * \mathrm{P}<0.001$ compared with the saline control group; one-way ANOVA followed by post-hoc Dunnett's tests. Pearson's $\mathrm{r}$ correlation test for correlation analysis.

A.

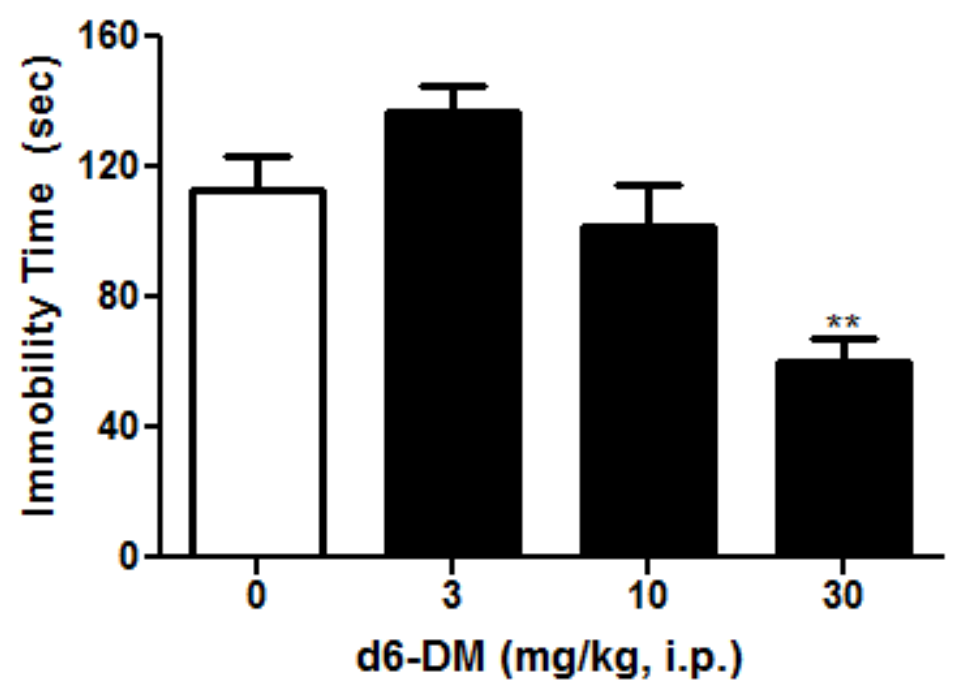

B.

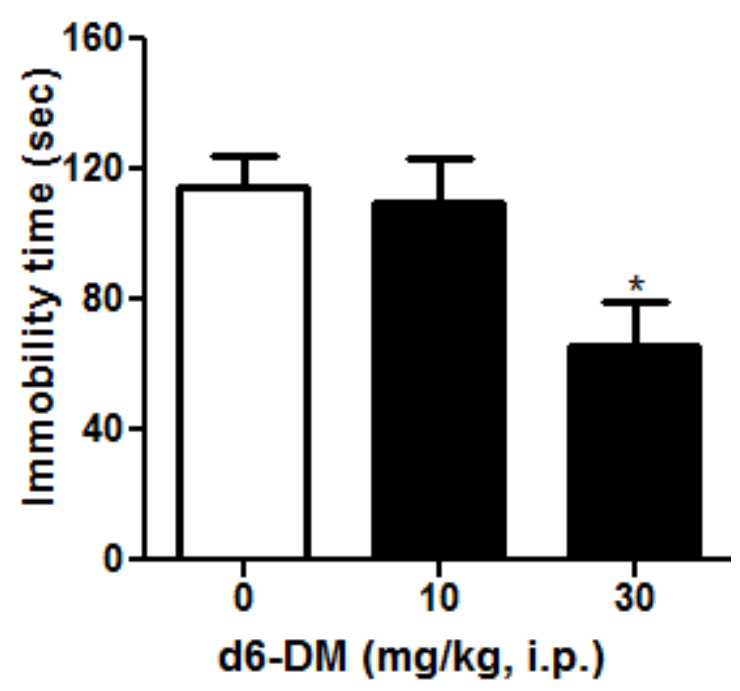



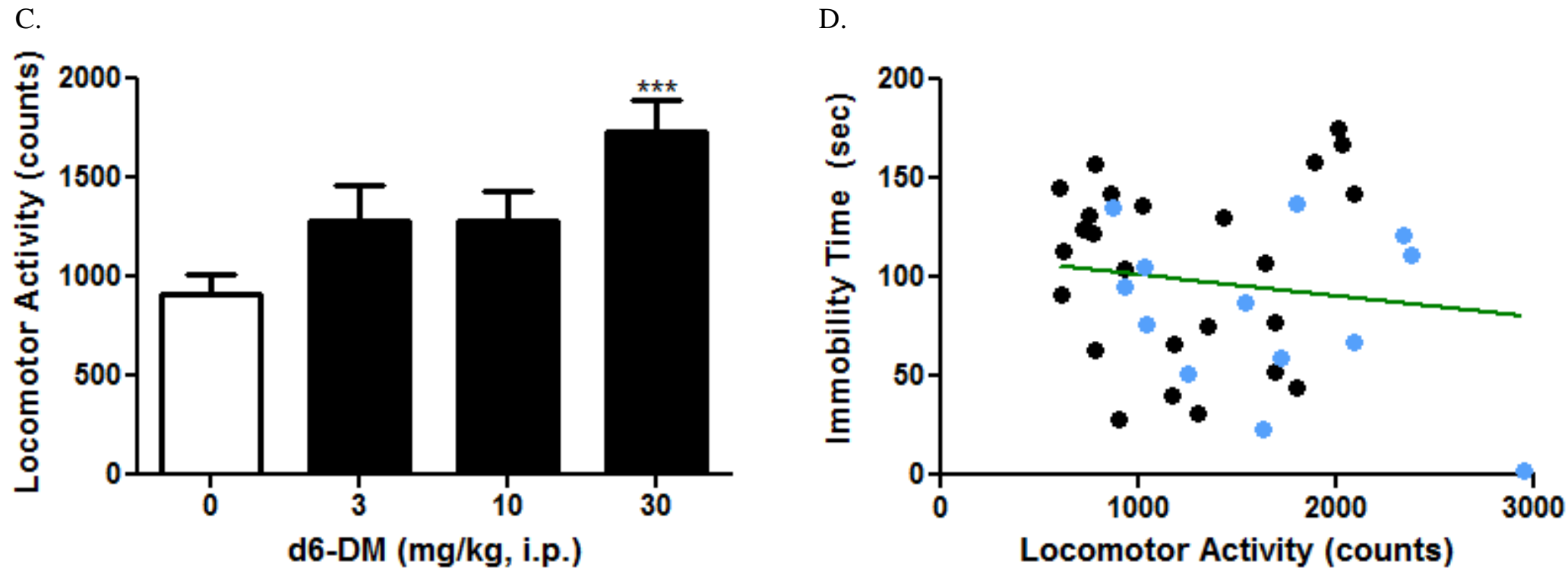
Figure 24. Blockade of AMPA or sigma-1 receptors attenuated the stimulatory effects, but not antidepressant-like effects of d6-DM. Pretreatment with NBQX (AMPA receptor antagonist, $30 \mathrm{mg} / \mathrm{kg}$ ), BD1063 (sigma-1 receptor antagonist, $10 \mathrm{mg} / \mathrm{kg}$ ), or BD1047 (sigma-1 receptor antagonist, $20 \mathrm{mg} / \mathrm{kg}$ ) failed to significantly block d6-DM (30 mg/kg)-induced reduction in immobility time in the FST (A, B, and C, respectively). In the OFT, NBQX, BD1063, and BD1047 all prevented the d6-DM-induced increase in locomotor activity (D, E, and F, respectively). Data shown are expressed as mean \pm SEM. $* \mathrm{P}<0.05$, $* * \mathrm{P}<0.01$, $* * * \mathrm{P}<0.001$, compared with the saline control group; \#\#\#P<0.001 compared to the d6-DM-treated group; one-way ANOVA followed by post-hoc Tukey’s tests.

A.

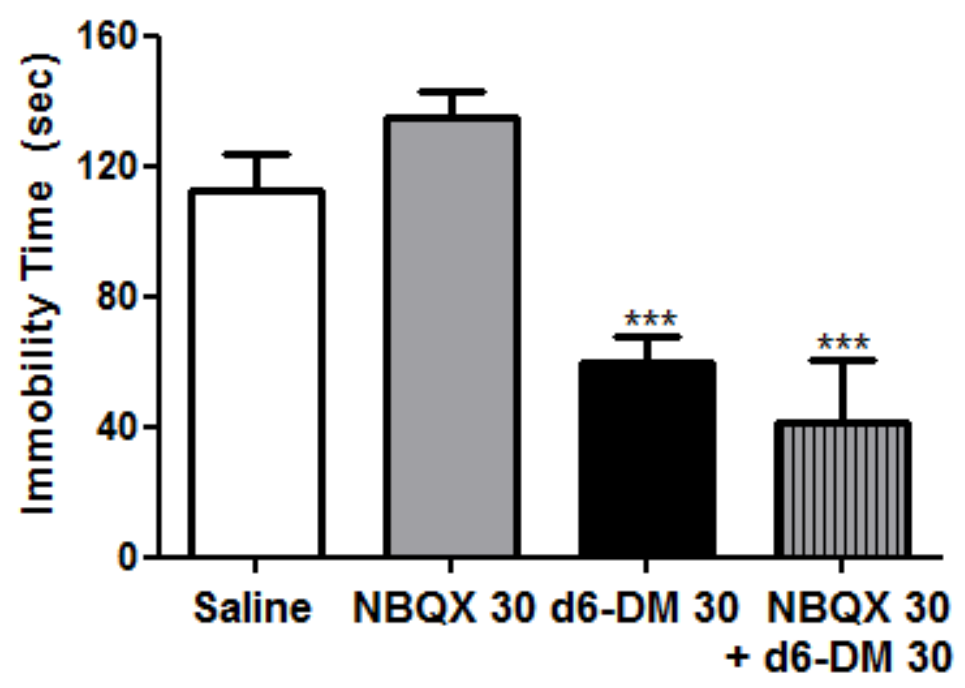

B.

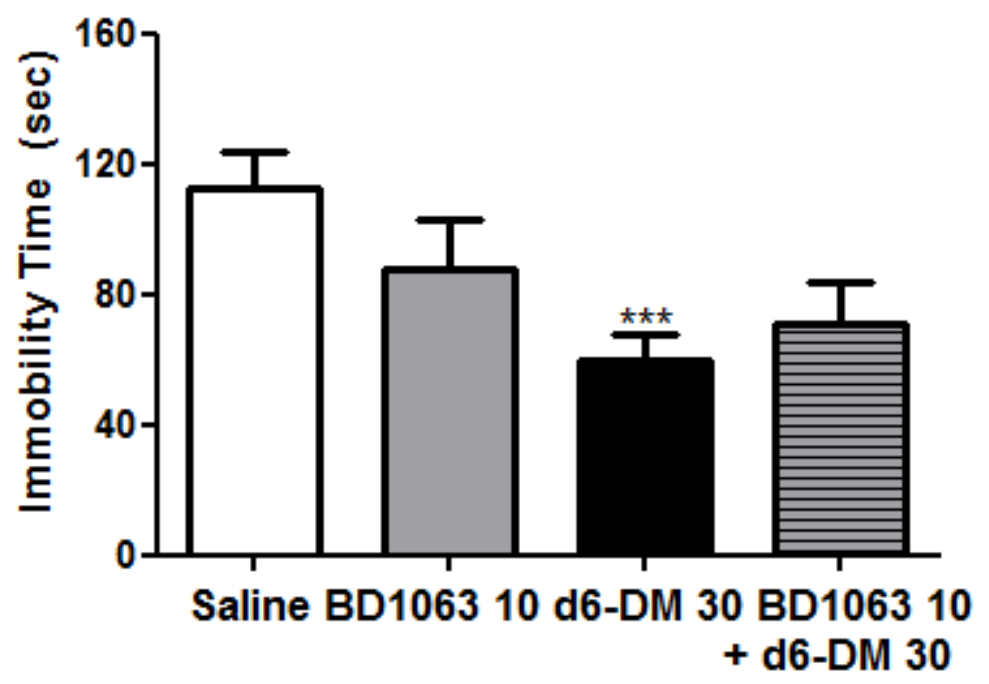



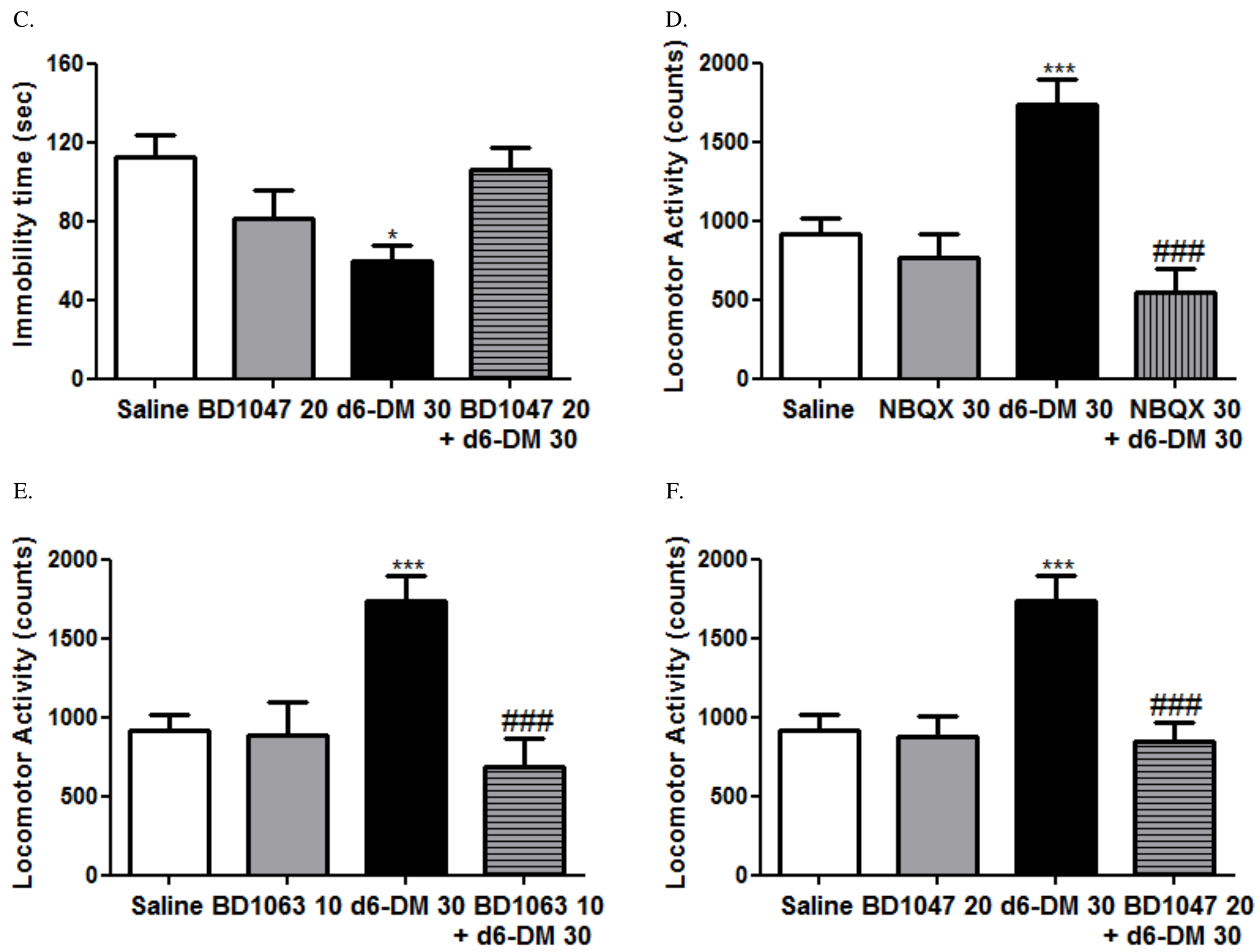
antidepressant-like effects of d6-DM (Figure 24B and 2C). The overall ANOVA was significant for the BD1063 and BD1047 pretreatment studies $(\mathrm{F}[3,46]=4.16, \mathrm{P}<0.05$, and $\mathrm{F}[3,37]=4.14, \mathrm{P}<0.05$, respectively). Post-hoc Tukey's tests further confirmed that the BD1063 + d6-DM and BD1047 + d6-DM groups did not different significantly from saline ( $q=3.55$, n.s. and $\mathrm{q}=0.60$, n.s., respectively). However, the difference in the effects of d6-DM in the absence and presence of BD1063 or BD1047 was not significant ( $q=0.82$, n.s. and $q=3.48$, n.s., respectively), reflecting partial attenuation of the effects. In the locomotor studies, there was a significant difference in the effects of the various treatment groups (Figure 24D, E, and F; F[3,61] $=11.36, \mathrm{P}<0.001 ; \mathrm{F}[3,66]=9.58, \mathrm{P}<0.001$ and $\mathrm{F}[3,66]=10.37, \mathrm{P}<0.001$, respectively). Post-hoc Tukey's tests confirmed the ability of pretreatment with NBQX, BD1063, or BD1047 to block the stimulant effect of d6-DM ( $\mathrm{q}=5.88, \mathrm{P}<0.001 ; \mathrm{q}=6.24, \mathrm{P}<0.001$, and $\mathrm{q}=5.77$, $\mathrm{P}<0.001$, respectively).

\subsubsection{Effects of quinidine on $\mathrm{d} 6-\mathrm{DM}$ in the FST and OFT}

Co-administration of quinidine $(30 \mathrm{mg} / \mathrm{kg})$ had no significant effects on the antidepressant-like effects of d6-DM (Figure 25A). The overall ANOVA was significant $(\mathrm{F}[7,77]=4.86, \mathrm{P}<0.001)$. Post-hoc Tukey's test confirmed that d6-DM (30 mg/kg) alone was significantly different from saline $(\mathrm{q}=4.92$, $\mathrm{P}<0.05)$ and not significantly different from Quinidine + d6-DM (30 mg/kg) $(\mathrm{q}=0.04, \mathrm{n} . \mathrm{s}$.$) . Though$ quinidine in combination with increasing doses of d6-DM produced very similar dose-dependent decreases in immobility time compared to d6-DM alone, the highest dose of d6-DM tested $(30 \mathrm{mg} / \mathrm{kg}) \mathrm{did}$ not produce a statistically significant effect when combined with quinidine compared to the saline control group ( $\mathrm{q}=3.83$, n.s.). In locomotor studies, quinidine significantly reduced the stimulatory effects of d6DM (30 mg/kg) (Figure 25B; F[7,102]=5.39, P<0.001). Post-hoc Tukey's tests confirmed that the d6-DM $(30 \mathrm{mg} / \mathrm{kg})$ alone group differed significantly from saline $(\mathrm{q}=6.99, \mathrm{P}<0.001)$ as well as the Quinidine + d6-DM (30 mg/kg) group (q=6.74, $\mathrm{P}<0.001)$. 
Figure 25. Co-administration of quinidine attenuated the stimulatory effects, but had no significant effects on the antidepressant-like effects of d6-DM. Co-administration of quinidine $(30 \mathrm{mg} / \mathrm{kg})$ produced a dose-dependent, albeit statistically non-significant, decrease in immobility time in combination with d6DM $(30 \mathrm{mg} / \mathrm{kg})$ in the FST (A). In the OFT, quinidine blocked the d6-DM-induced increase in locomotor activity (B). Data shown are expressed as mean \pm SEM. $* \mathrm{P}<0.05$, $* * * \mathrm{P}<0.001$, compared with the saline control group; \#\#\#P<0.001 compared to the d6-DM (30 mg/kg)-treated group; one-way ANOVA followed by post-hoc Tukey's tests. Black bar ( $\square)$ with quinidine; white bar ( $\square$ ) without quinidine.

A.
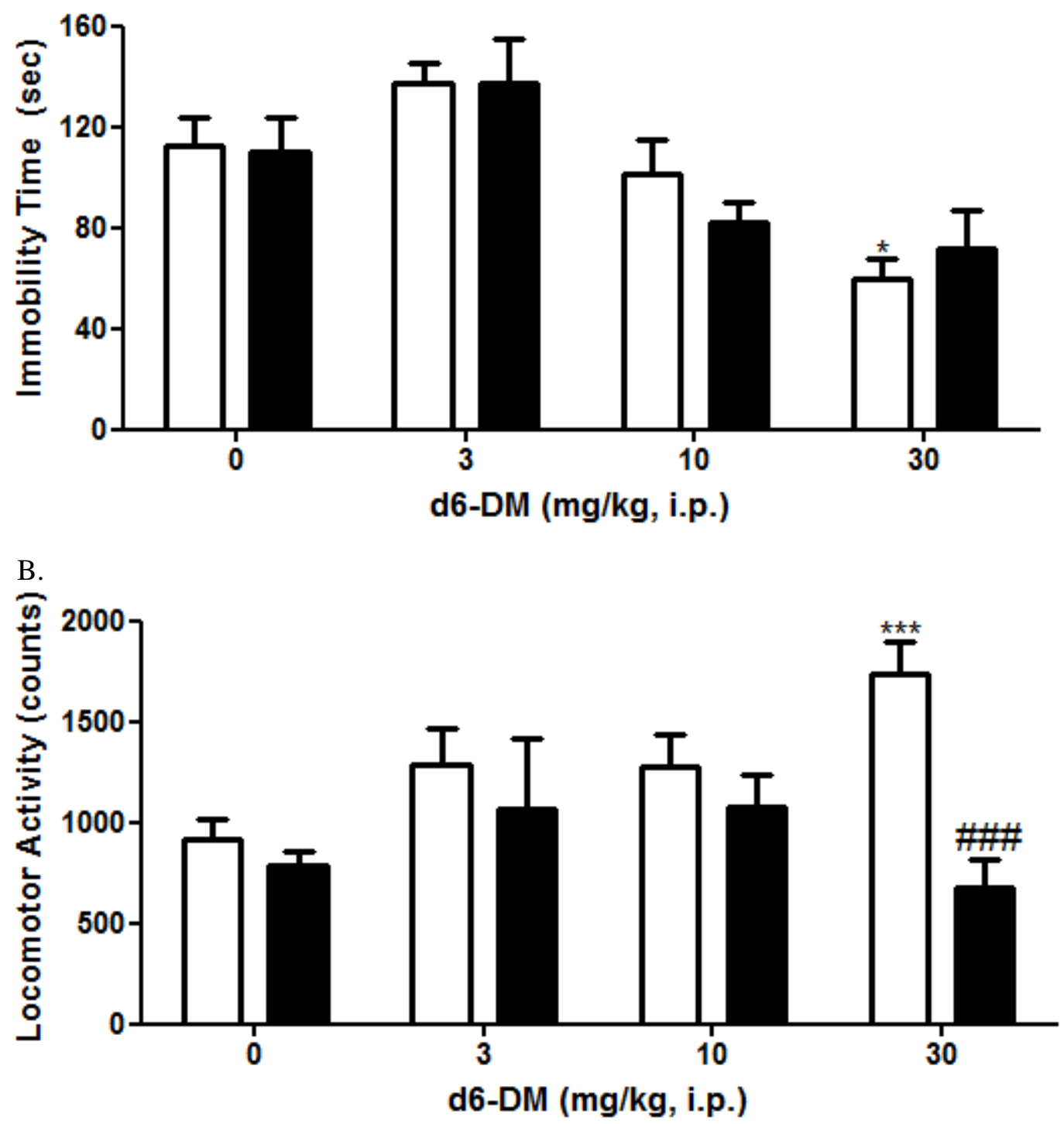


\subsection{Discussion}

d6-DM displays a dose-dependent decrease in immobility time in the FST and TST similar to regular DM, reaching significance at the same dose (Nguyen and Matsumoto, 2015; Nguyen et al., 2014). Concomitant administration of quinidine appears to have no significant effects on d6-DM in the FST, suggesting that any additional metabolism of d6-DM that may be occurring in vivo is unlikely to significantly alter its antidepressant-like actions. Interestingly, while pretreatment with an AMPA receptor antagonist (NBQX) or a sigma-1 receptor antagonist (BD1063) had previously significantly attenuated the antidepressant-like effects of DM in the FST (Nguyen and Matsumoto, 2015; Nguyen et al., 2014), pretreatment with these same doses of antagonists failed to significantly block the antidepressant-like effects of d6-DM, suggesting that d6-DM may be working through mechanisms different from DM to facilitate its antidepressant actions.

The inability of the antagonists to block the antidepressant-like effects of regular DM but not d6DM is surprising, considering that deuteration of a compound typically has no effect on biological potency or selectivity (e.g., target binding affinity, subtype binding) (Gant, 2014). In fact, it is often virtually identical in all of its biological properties with the useful exception of those processes involving bond-breaking at the specifically modified C-H site(s) that are oxidatively labile (Gant, 2014). The C-d6 bonds may be considerably more stable to oxidative processes, rendering the d6-enriched compound to be more resistant to, for instance, CYP-mediated metabolism (Gant, 2014). Preliminary binding affinity studies for d6-DM as well as pharmacokinetic data in healthy human subjects suggest these general features of d6-compounds are also true of d6-DM: d6-DM had virtually indistinguishable binding affinities from DM at all tested protein targets, which included many of the antidepressant-relevant ones mentioned below, and it also had a longer half-life (L. Pope, personal communication). d6-DM is expected to similarly have a longer half-life in mice, though this remains to be confirmed. With improved metabolic stability, it is likely that the specific types and ratios of metabolites produced by d6-DM differs from regular DM and convey a different biological profile. This may in part explain why d6-DM is seen here producing antidepressant-like effects through a different mechanism than regular DM. 
DM has multiple properties in common with fast acting and conventional antidepressants as well as unique properties that may confer therapeutic response in depression, especially in the treatment of refractory cases (Lauterbach, 2012; Stahl, 2013b). On top of utilizing AMPA and sigma-1 receptors to convey antidepressant effects, for example, DM may increase 5-HT levels (Codd et al., 1995; Kamei et al., 1992), possibly through its interaction at SERT and 5-HT1B/D receptor (Codd et al., 1995; Werling et al., 2007a). DM has also been reported to modulate NE reuptake (Codd et al., 1995), which would be anticipated to also contribute antidepressant effects in humans, although it would not account for potential fast acting effects. In addition, similar to ketamine, DM is an NMDA receptor antagonist, which is thought to be an initiating mechanism by which ketamine produces its fast acting effects (Abdallah et al., 2015). Thus, the multiple receptor actions of DM and its ability to modulate the monoaminergic and glutamatergic system suggests that it would have, if not rapid acting effects, at least some beneficial effects in depressed patients. Indeed, a recent retrospective study has shown that DM in combination with quinidine can improve mood in depressed patients with treatment-resistant bipolar disorder, with some reporting changes within 1-2 days of initiating treatment or increasing dose (Kelly and Lieberman, 2014). It is possible that d6-DM may be using these other described mechanisms of action, or that all these mechanisms are contributing to the antidepressant-like effects of d6-DM observed in the FST such that no one antagonist can inhibit the effects alone. In support of the latter scenario, the sigma-1 receptor antagonists, especially BD1047, produced a noticeable, albeit not statistically significant, trend toward the prevention of the decreased immobility time induced by d6-DM. This suggests that sigma-1 receptors may play a partial role in producing the antidepressant-like effects of d6-DM. Moreover, it is important to note that we only used one depression-related behavioral assay in the antagonism studies. Though the FST is the most validated animal model for predicting antidepressant efficacy and thus provides a rational format for initial evaluation of the antidepressant potential of d6-DM, additional studies are needed to further examine this antidepressant potential and the involved mechanism(s) of action.

Finally, d6-DM produces stimulant actions that were quantified herein as increases in locomotor activity. Two observations are of note with regard to these actions. First, the stimulant effects cannot 
account for the antidepressant-like actions of d6-DM. Second, quinidine does not appear to alter the antidepressant-like effects of d6-DM but significantly reduced the d6-DM-induced increase in locomotor activity. The latter finding suggests certain metabolites (most likely DX) are still being formed with d6DM alone (in the absence of quinidine) and may be contributing to the observed stimulant actions. The addition of quinidine to d6-DM therefore could be advantageous during further drug development since the combination may retain desired therapeutic antidepressant-like effects without eliciting unwanted stimulant effects. This reduction in stimulatory effects and potential abuse liability is especially important considering recent reports of a rise in the abuse of DM in OTC cough medicines, especially by adolescents (Bryner et al., 2006; Burns and Boyer, 2013).

In conclusion, the present data show that deuteration of DM retains antidepressant efficacy, though this modification may lead to the recruitment of different protein target(s) through altered metabolism profiles, compared to regular DM, to elicit the antidepressant actions. Moreover, the addition of quinidine to further inhibit the metabolism of d6-DM did not significantly affect its antidepressant-like effects but removed unwanted stimulant effects. Additional refinements of the deuteration strategy may improve therapeutically effective blood levels of DM without even the need of quinidine or lower doses of quinidine than currently used in combination with regular DM for the treatment of PBA (Schoedel et al., 2014); for though the dose of quinidine used for PBA (10 mg) is very low relative to doses used for its standalone approved indications $(100-600 \mathrm{mg})$, there are still concerns about its ability to change the CYP2D6-mediated metabolism of many other drugs (e.g., aripiprazole, donepezil, fluoxetine and paroxetine) (Kelly and Lieberman, 2014; Schoedel et al., 2014; Schoedel et al., 2012) and caution and contraindications to be considered in patients with a history of cardiovascular disease (e.g., risk for QTprolongation and heart failure) (Schoedel et al., 2014). Overall, d6-DM appears to be a promising antidepressant drug lead, possibly with rapid acting antidepressant effects or efficacy in treatmentresistant cases, with improved metabolic stability compared DM. 
Acknowledgments: We appreciate the technical support of Anna Scandinaro, who was supported by a Summer Undergraduate Research Fellowship (SURF) from the American Society of Pharmacology and Experimental Therapeutics (ASPET). Unpublished data about d6-DM was provided by Dr. Laura Pope (Avanir Pharmaceuticals, Inc.) to facilitate the design of the studies and interpretation of the data. Part of the data was presented at the Experimental Biology 2015 annual meeting: Scandinaro, A. Nguyen, L., Matsumoto R. (2015). Modified dextromethorphan displays antidepressant-like effects in the forced swim test. ABSTRACT, The FASEB Journal 29:931.1. 


\section{CHAPTER 8}

Evaluation of the antidepressant-like effects of dextrorphan 


\subsection{Introduction}

DM is rapidly metabolized primarily by CYP2D6 into its major metabolite DX (Capon et al., 1996; Schmid et al., 1985; Yu and Haining, 2001). Because of this metabolism, the relative extent to which DM or DX mediates the effects of DM is not always clear. This in turn has a bearing on the mechanism of action of DM in view of the different affinities of DM and DX for various receptor sites (Werling et al., 2007a).

In the present study, we examined whether the primary metabolite per se had antidepressant-like effects. Using the FST and TST, the two most validated behavioral assays for assessing antidepressant potential (Cryan and Holmes, 2005; Cryan et al., 2005; McArthur and Borsini, 2006; Nestler et al., 2002b), DX was evaluated for antidepressant-like effects. Next, as sigma-1 receptors and AMPA have been implicated in mediating the antidepressant-like actions of DM, two sigma-1 receptor antagonists (BD1063 and BD1047) and an AMPA receptor antagonist (NBQX) were evaluated in conjunction with DX to determine the involvement of these two receptors in the antidepressant-like effects of DX.

\subsection{Results}

\subsubsection{Effects of DX in the FST, TST, and OFT}

DX produced a significant dose-dependent decrease in immobility time in the FST and TST (Figure 26A and B; F[3,36]=6.46, $\mathrm{P}<0.01$ and $\mathrm{F}[2,19]=3.68, \mathrm{P}<0.05$, respectively). Post-hoc Dunnett's test showed that DX at $40 \mathrm{mg} / \mathrm{kg}$ was significantly different from saline $(\mathrm{q}=3.86, \mathrm{P}<0.01$ and $\mathrm{q}=2.69$, $\mathrm{P}<0.05$, in the FST and TST respectively). In the OFT, DX also produced a significant increase in locomotor activity (Figure 26C; F[3,58]=16.62, P<0.0001). Post-hoc Dunnett's tests confirmed that the following doses of DX significantly differed from saline: 20 ( $\mathrm{q}=2.91, \mathrm{P}<0.05), 30(\mathrm{q}=6.82, \mathrm{P}<0.001)$, and $40(\mathrm{q}=3.71, \mathrm{P}<0.01) \mathrm{mg} / \mathrm{kg}$. Since DX showed significant stimulant effects, a correlation analysis between locomotor activity and immobility time was carried out to determine whether the stimulant effects could account for its observed antidepressant-like actions. The Pearson's r correlation test including data points from all the doses of DX tested revealed that there was no correlation between the 
Figure 26. Antidepressant-like and stimulatory effects of DX in the FST, TST and OFT. DX produced a significant decrease in immobility time the FST (A) and FST (B) and a significant increase in locomotor activity in the OFT (C). However, no significant correlation was observed

between the d6-DM-induced alterations in locomotor activity and immobility time (D). Bar graph data shown are expressed as mean \pm SEM. For the correlation graph, black circles represent data points from mice subjected to the FST and blue circles from mice subjected to the TST. *P $<0.05$, $* * \mathrm{P}<0.01, * * * \mathrm{P}<0.001$ compared with the saline control group; one-way ANOVA followed by post-hoc Dunnett's tests. Pearson's $\mathrm{r}$ correlation test for correlation analysis.

$$
\text { A. }
$$

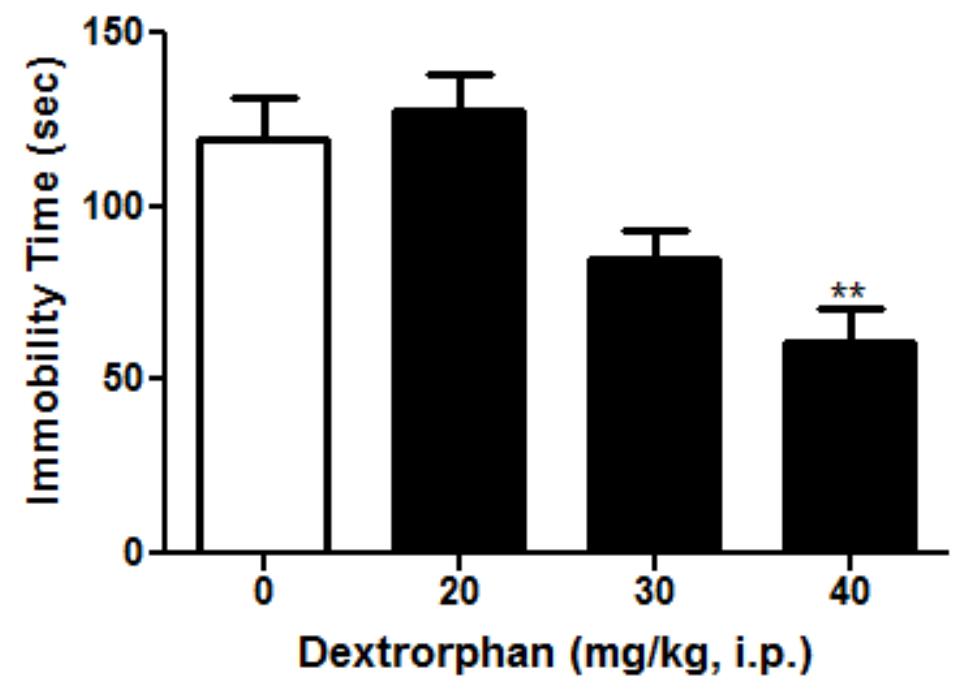

B.

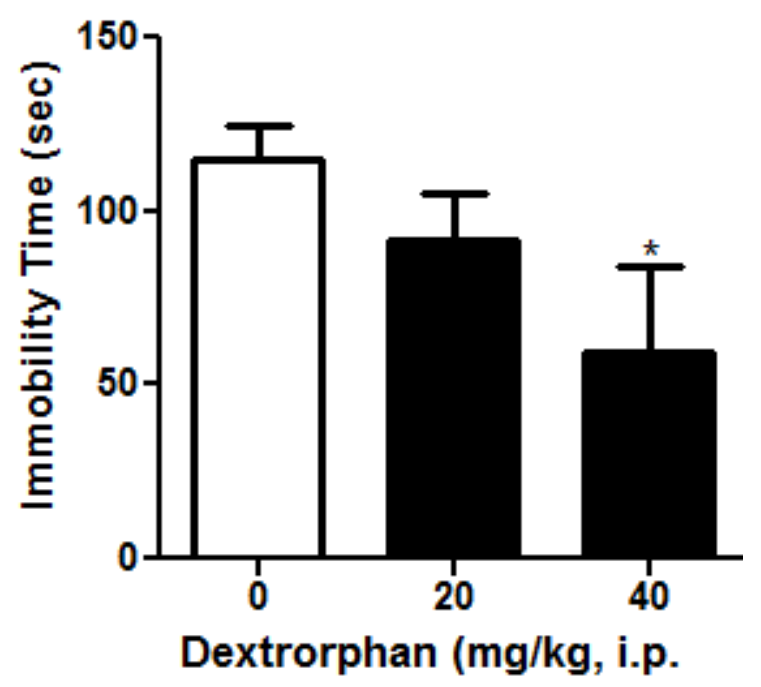


C.

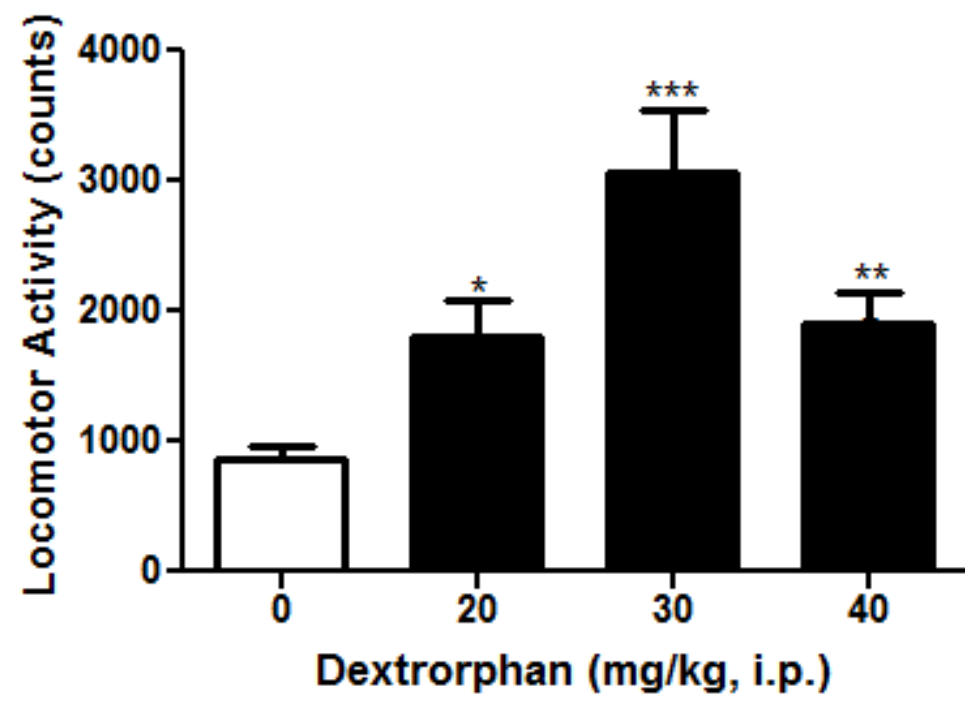

D.

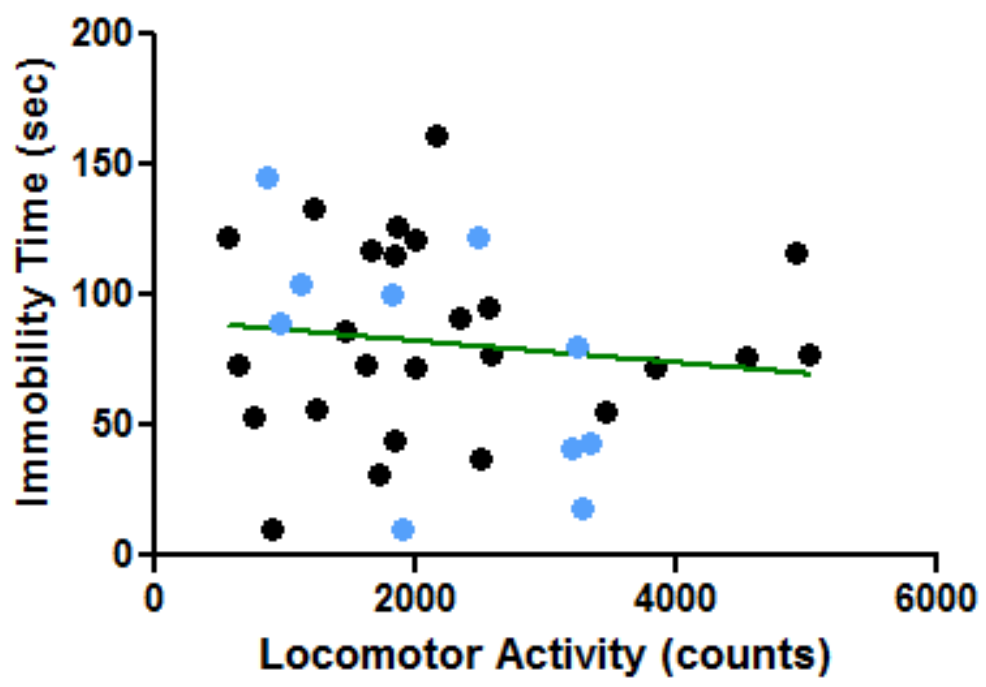


DX-induced alterations in locomotor activity in the OFT and immobility time in the FST and TST (Figure $25 \mathrm{D} ; \mathrm{r}=-0.13$, n.s.).

\subsubsection{Effects of sigma-1 receptor antagonists on DX in the FST and OFT}

Pretreatment with the sigma-1 preferring antagonists BD1063 (10 mg/kg) or BD1047 (20 mg/kg) failed to attenuate antidepressant-like effects of DX in the FST (Figure 27A and B, respectively). ANOVA confirmed a significant difference between the various treatment groups for the BD1063 $(\mathrm{F}[3,36]=4.52, \mathrm{P}<0.01)$ and $\mathrm{BD} 1047(\mathrm{~F}[3,36]=4.25, \mathrm{P}<0.05)$ antagonism studies. Pairwise comparisons using post-hoc Tukey's multiple comparison tests confirmed that DX significantly reduced immobility time compared to saline ( $\mathrm{q}=4.60, \mathrm{P}<0.05$ and $\mathrm{q}=4.71, \mathrm{P}<0.05$, respectively). However, post-hoc comparisons showed that neither the BD1063 + DX nor the BD1047 + DX groups differed significantly from DX alone (q=0.11, n.s. and q=0.69, n.s., respectively). Similarly, in the locomotor studies, the overall ANOVA was significant for the BD1063 and BD1047 pretreatment studies (Figure 27C and D; $\mathrm{F}[3,36]=10.87, \mathrm{P}<0.0001$ and $\mathrm{F}[3,36]=8.00, \mathrm{P}<0.001$, respectively). Post-hoc Tukey's tests confirmed that DX produced stimulatory effects $(\mathrm{q}=6.08, \mathrm{P}<0.001$ and $\mathrm{q}=5.80, \mathrm{P}<0.01$, respectively). However, the BD1063 + DX and BD1047 + DX groups also differed significantly from saline (q=6.08, P<0.001 and $\mathrm{q}=4.03, \mathrm{P}<0.05$ respectively), reflecting failed attenuation of the stimulatory effects of DX. Neither BD1063 nor BD1047 alone had significant effects in the FST ( $\mathrm{t}=1.59$, n.s.; $\mathrm{t}=95$, n.s., respectively) or OFT (t=1.30, n.s.; $\mathrm{t}=1.69$, respectively).

\subsubsection{Effects of AMPA receptor antagonist on DX in the FST, TST and OFT}

Pretreatment with the potent AMPA receptor antagonist NBQX (10 or $30 \mathrm{mg} / \mathrm{kg}$ ) failed to attenuate antidepressant-like effects of DX in the FST and TST (Figure 28A and B, respectively). ANOVA confirmed a significant difference between the various treatment groups for the NBQX antagonism studies in the FST $(\mathrm{F}[5,49]=4.62, \mathrm{P}<0.01)$ and TST $(\mathrm{F}[3,28]=7.37, \mathrm{P}<0.001)$. Pairwise 
Figure 27. Blockade of sigma-1 receptors failed to attenuate the antidepressant-like effects of DX in the FST (A and B) and stimulatory effects in the OFT (C and D). Data are expressed as mean \pm SEM. ${ }^{*} \mathrm{P}<0.05$, $* * \mathrm{P}<0.01$, $* * * \mathrm{P}<0.001$ vs. saline-treated group; one-way ANOVA followed by post-hoc Tukey's tests. DX, dextrorphan.

A.

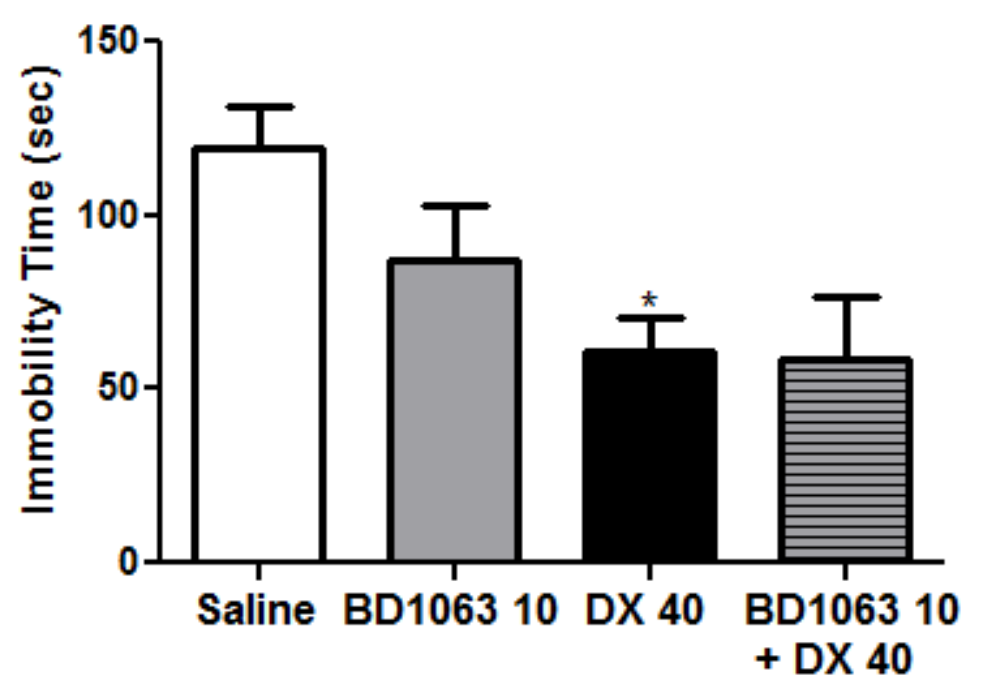

B.

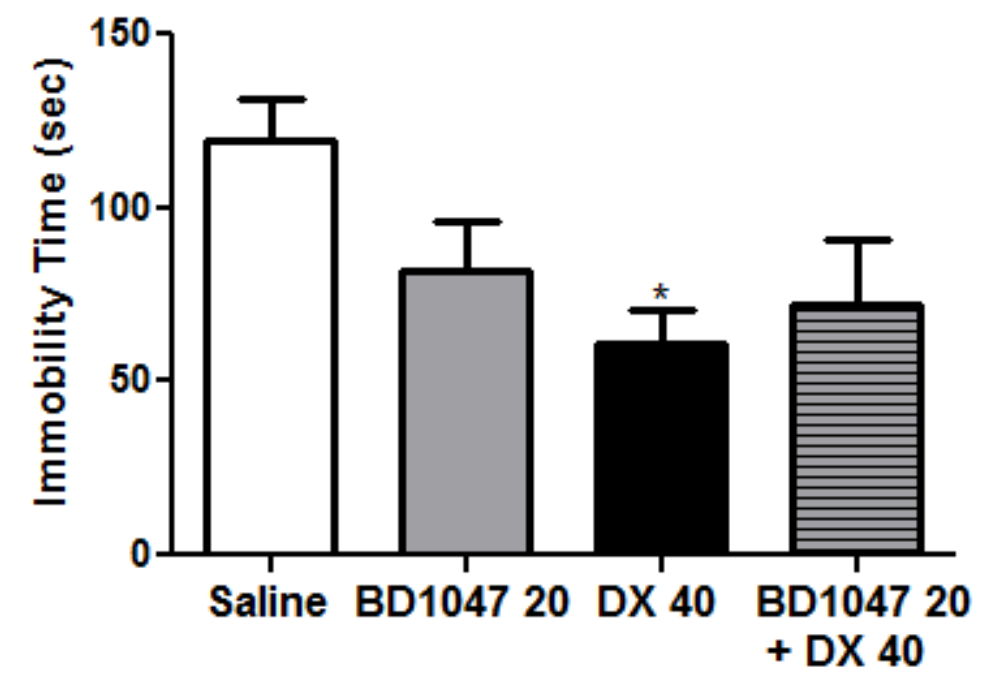



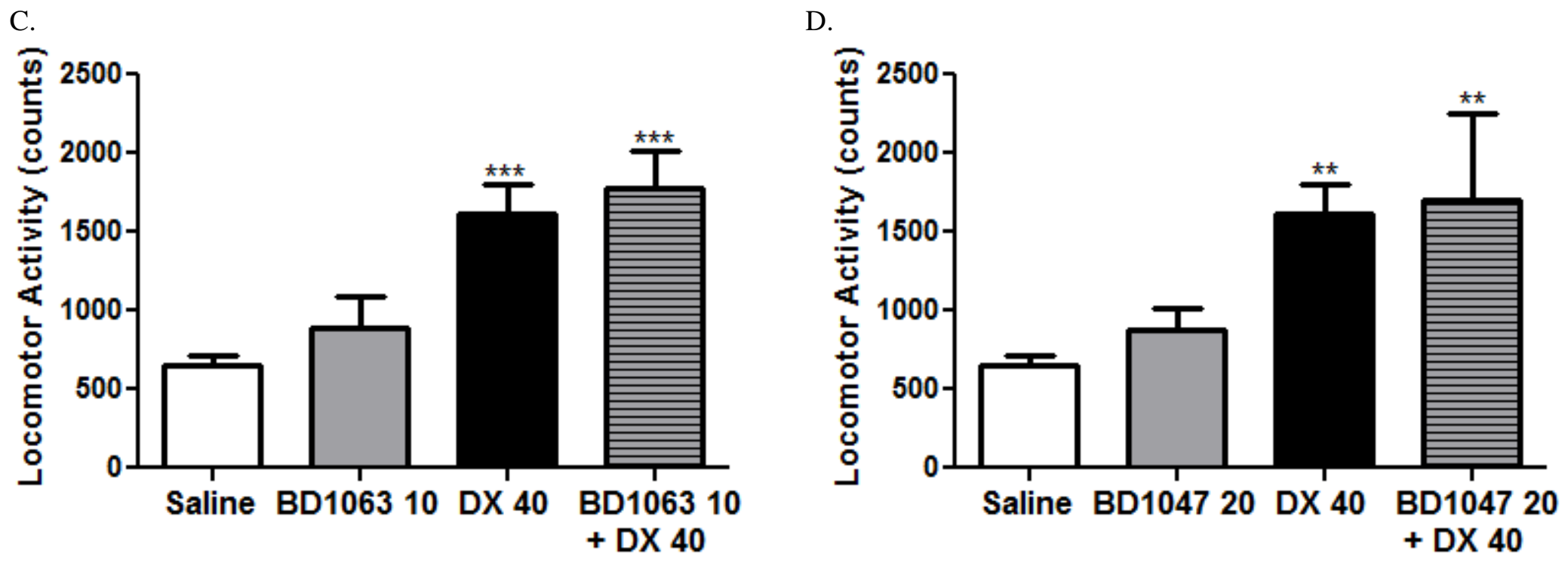
Figure 28. Blockade of AMPA receptors failed to attenuate the antidepressant-like effects of DX in the FST and TST (A and B, respectively), but blocked the stimulatory effects in the OFT (C). Data are expressed as mean \pm SEM. $* \mathrm{P}<0.05, * * \mathrm{P}<0.01$, vs. saline-treated group; $\# \mathrm{P}<0.05$, vs. DX-treated group; one-way ANOVA followed by post-hoc Tukey's tests. DX, dextrorphan.

A.

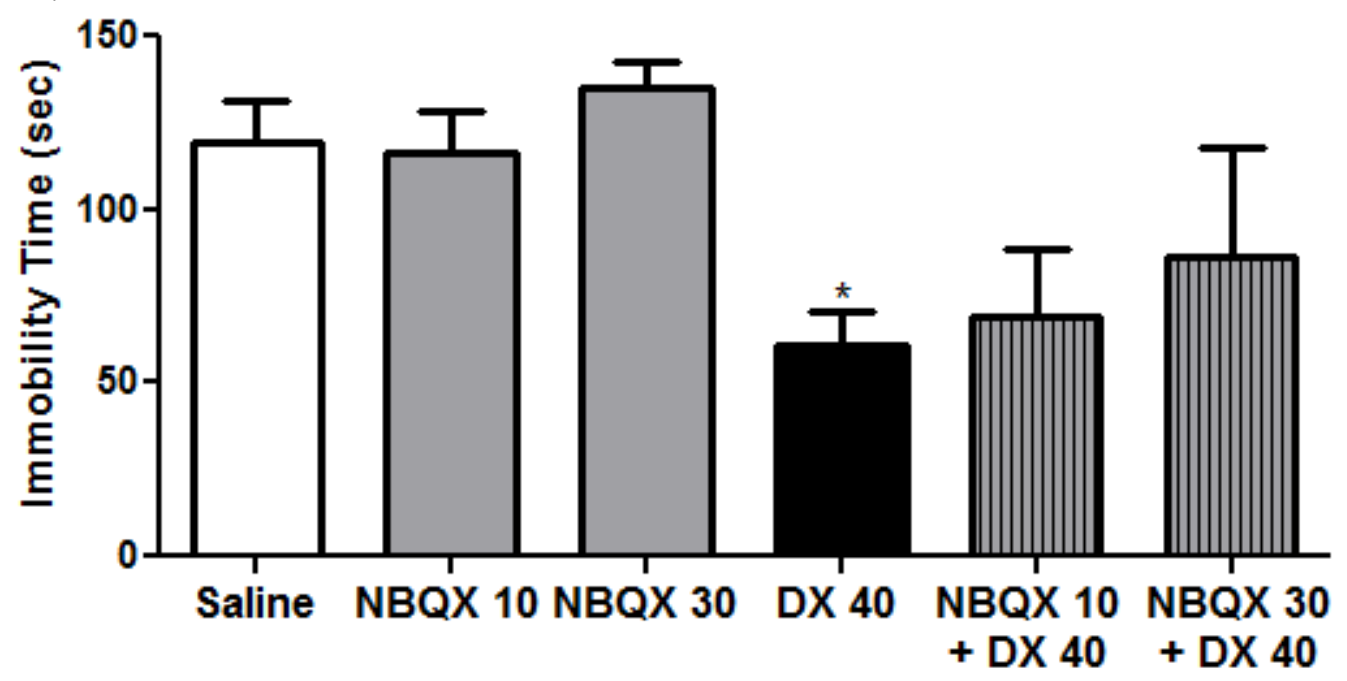

B.

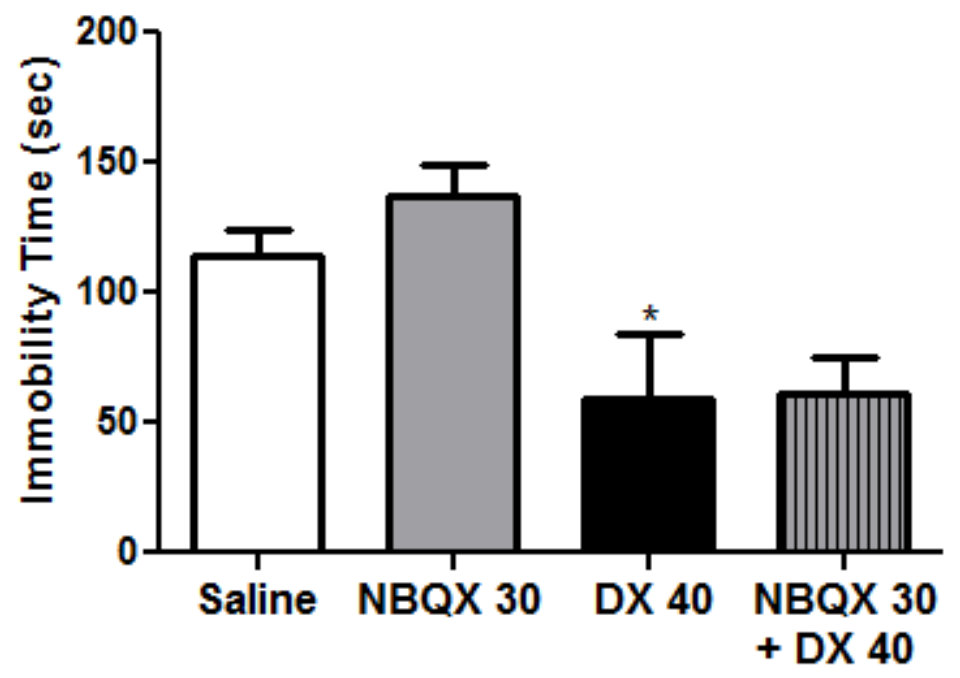




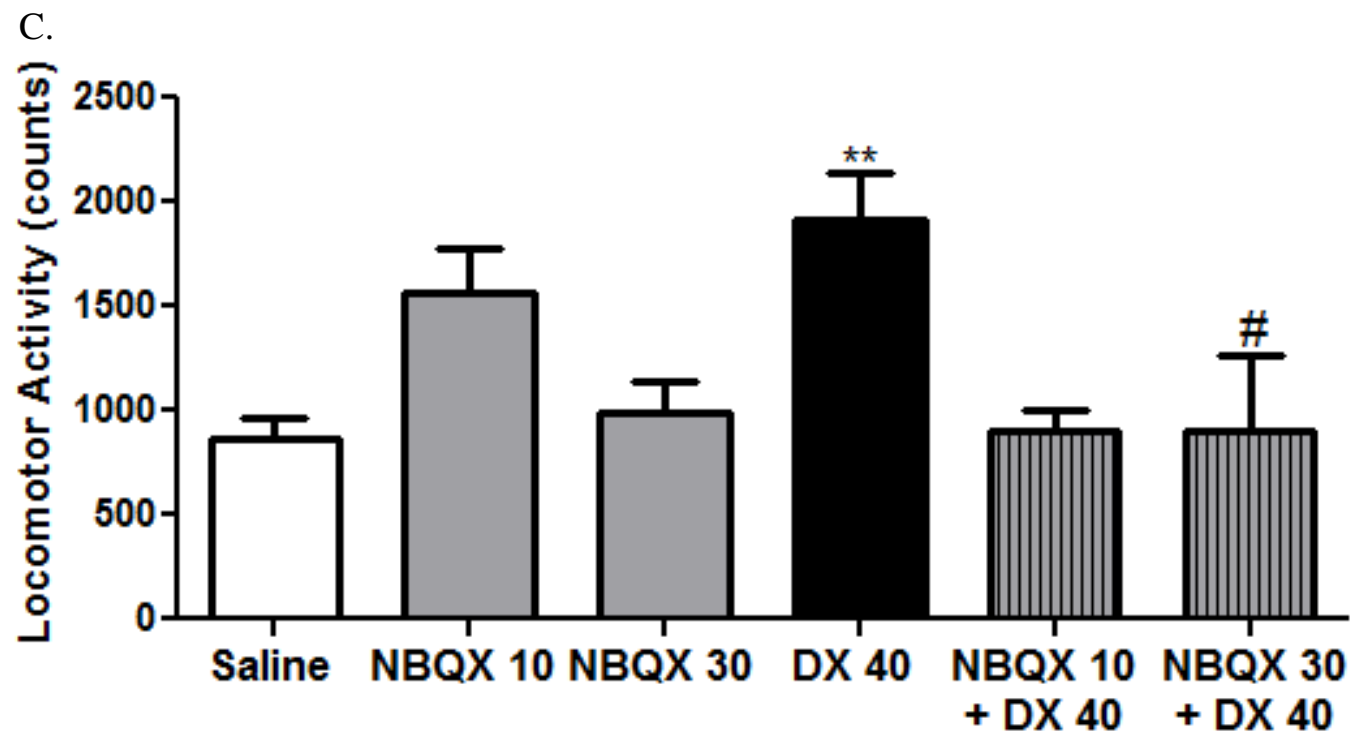


comparisons using post-hoc Tukey's multiple comparison tests confirmed that DX significantly reduced immobility time compared to saline in the FST and TST ( $q=4.81, \mathrm{P}<0.05$ and $\mathrm{q}=3.87, \mathrm{P}<0.05$, respectively). However, post-hoc comparisons showed that NBQX (10 mg/kg) + DX or NBQX (30 $\mathrm{mg} / \mathrm{kg})+\mathrm{DX}$ in the FST and NBQX $(30 \mathrm{mg} / \mathrm{kg})+\mathrm{DX}$ in the TST did not differ significantly from DX alone ( $\mathrm{q}=0.54$, n.s.; $\mathrm{q}=1.58$, n.s.; and $\mathrm{q}=0.10$, n.s., respectively). In the locomotor studies, pretreatment with NBQX mitigated the stimulatory effects of DX (Figure 28C; F[5,81] =5.56, P<0.001). Post-hoc Tukey's tests confirmed that DX produced stimulatory effects $(\mathrm{q}=6.46, \mathrm{P}<0.001)$, and the NBQX (30 $\mathrm{mg} / \mathrm{kg})+\mathrm{DX}$ group differed significantly from the DX group $(\mathrm{q}=4.92, \mathrm{P}<0.05)$. NBQX $10 \mathrm{mg} / \mathrm{kg}$ alone had significant effects in the OFT $(\mathrm{q}=3.23, \mathrm{P}<0.01)$, but neither doses had significant effects in the FST $(\mathrm{F}[2,32]=0.68$, n.s. $)$ or $\operatorname{TST}(\mathrm{F}[2,29]=2.93$, n.s. $)$.

\subsection{Discussion}

DX displays a dose-dependent decrease in immobility time in the FST and TST, similar to the parent compound DM (Nguyen and Matsumoto, 2015; Nguyen et al., 2014). However, pretreatment with neither the sigma-1 receptor antagonists nor the AMA receptor antagonist could attenuate the antidepressant-like effects of DX, suggesting that DX may be eliciting antidepressant-like behaviors through distinct mechanism(s).

DX, similar to DM, has multiple properties in common with fast acting and conventional antidepressants as well as unique properties which may confer therapeutic response in depression, especially in the treatment of refractory cases (Lauterbach, 2012). In addition to binding to sigma-1 receptors, DX may increase 5-HT levels (Codd et al., 1995), possibly through its interaction at SERT and 5-HT1B/D receptor (Codd et al., 1995; Werling et al., 2007a). DX has also been reported to modulate NE reuptake (Codd et al., 1995), which would be anticipated to also contribute antidepressant effects in humans. In addition, like ketamine, DX is a potent non-competitive NMDA receptor antagonist, with approximately 10-fold greater binding affinity to the PCP-binding site than DM (Chou et al., 1999; Kim et al., 2003a; Shin et al., 2007; Werling et al., 2007a). Thus, with its multiple receptor actions and 
potential ability to modulate the monoaminergic and glutamatergic system, it is possible that DX may be using these other described mechanisms of action, or that all these mechanisms are contributing to the antidepressant-like effects of DX observed in the FST and TST such that no one antagonist can inhibit the effects alone.

Possibly by recruiting different protein(s) to elicit antidepressant actions, exposure to DX may contribute to the antidepressant potential of DM. However, we have previously shown that quinidine potentiated the antidepressant-like effects of DM in vivo (Nguyen and Matsumoto, 2015; Nguyen et al., 2014) and this combination has been shown to rapidly resolve depressive symptoms in some bipolar depressed patients (Kelly and Lieberman, 2014), suggesting that the antidepressant-like properties of DM may be enhanced with increased early exposure of the parent compound (DM) compared to the major metabolites (DX). In addition, because neither sigma-1 receptors nor AMPA receptors, which are two molecular targets associated with producing a faster onset of antidepressant action (Hayashi and Su, 2008; Knapp et al., 2002; Koike et al., 2011), appear to play an important role in mediating the antidepressantlike effects of DX, this suggests DX is unlikely to have fast acting antidepressant effects.

DX also produced stimulant actions which were quantified herein as increases in locomotor activity. Two observations are of note with regard to these actions. First, the stimulant effects cannot account for the antidepressant-like actions of DX. Second, our lab previously showed that DM can produce stimulatory effects in some cohort of animals (Nguyen et al., 2014) but not others (Nguyen and Matsumoto, 2015). Importantly, concomitant administration of quinidine to inhibit the rapid metabolism of DM and reduce exposure to DX enhanced the antidepressant-like effects of DM without producing an increase in locomotor activity (Nguyen and Matsumoto, 2015; Nguyen et al., 2014). In human studies, CYP2D6 activity appears to be an important factor in the psychoactive effects of high doses of DM: extensive metabolizers have reported greater abuse potential (e.g., higher ratings on the visual analog scales of "good" drug effects and drug "liking") (Zawertailo, Kaplan et al. 1998); and in a follow-up study, pretreatment with quinidine decreased the positive subjective effects such as euphoria and drug liking (Zawertailo, Tyndale et al. 2010). Likewise, increased exposure to DX have been thought to 
increase the risk for PCP-like psychotomimetic effects seen with high dose DM (Miller, 2011). These findings together suggest that DX may contribute to the stimulatory effects and abuse liability of DM observed in our in vivo studies (Nguyen et al., 2014) and in human populations (Bryner et al., 2006; Burns and Boyer, 2013; Miller, 2011). In addition, NMDA antagonists have not fared well in clinical trials despite promising preclinical data due a number of limiting adverse CNS effects, including hallucinations, a centrally mediated increase in blood pressure and, at high doses, catatonia and anesthesia (for which the NMDA ion-channel blockers PCP and ketamine were initially developed) (Kemp and McKernan, 2002). In this study, DX, along with stimulatory effects, produced notable ataxia at the antidepressant dose, indicative of problematic NMDA-related side effects (Muir and Lees, 1995). In conclusion, the present data show that DX produces antidepressant-like effects through mechanism(s) independent of sigma-1 and AMPA receptors. While it is still not fully understood the extent to which DX may mediate the effects of DM, studies utilizing quinidine to slow down the metabolism of DM thus far suggest that increased early exposure of the DM compared to DX may increase the antidepressant potential of DM, particularly for fast acting effects; and that avoiding significant metabolism to DX would enhance the therapeutic potential of DM by reducing potential side effects. Overall, DX may contribute to the antidepressant effects of DM, though exposure should be limited to decrease abuse liability and other adverse effects. 


\section{CHAPTER 9}

Summary and conclusions 
DM produces behavioral changes in both the FST and TST predictive of antidepressant efficacy. The antidepressant-like effects involve sigma-1 and AMPA receptors, are not dependent on stimulant actions, and also do not appear to be associated with changes in BDNF-related outputs. DX may contribute to the antidepressant effects of DM, though concomitant administration of quinidine to slow down the metabolism of DM and reduce exposure to DX have suggested that the antidepressant-like properties of DM is enhanced with increased early exposure of the parent compound (DM) compared to the major metabolites (DX). Moreover, reduced exposure to DX may improve the therapeutic potential of DM by reducing abuse liability and other adverse effects, most notably PCP-like psychotomimetic effects seen with high dose DM. Another approach to extend the half-life of DM is to chemically modify it to make it more resistant to metabolism, as in the case with d6-DM. d6-DM appears to be a promising antidepressant drug, with improved metabolic stability compared DM, though this modification may lead to the recruitment of different protein target(s) to elicit the antidepressant actions.

Ketamine, on the other hand, produced antidepressant-like effects that involved AMPA but not sigma-1 receptors, and were associated with an increase in pro-BDNF in the mouse hippocampus. Also important, imipramine, which unlike ketamine takes weeks instead of hours to produce antidepressant effects, displayed antidepressant-like effects that involved neither AMPA nor sigma-1 receptors and were not associated with an increase in pro-BDNF.

Overall, DM may share some, but not all antidepressant mechanisms with ketamine and imipramine, suggesting it may be useful particularly in treatment-refractory cases. Because the antidepressant actions of DM appear to involve both sigma-1 and AMPA receptors, two molecular targets associated with producing a faster onset of antidepressant actions in preclinical studies, it is likely that DM may produce antidepressant effects faster than conventional antidepressant. Compared to ketamine, DM may promote neural plasticity at a later time point or through trophic factors other than BDNF (e.g., NGF). In fact, there has been some clinical evidence that DM in combination with quinidine can rapidly improve mood in patients with treatment-resistant bipolar depression (Kelly and Lieberman, 2014). Whether or not DM will have rapid efficacy in unipolar depression as well remains to be determined. 
Fortunately, the availability of DM for use in humans both alone in combination with quinidine makes it amenable for repurposing and quick translation into the clinic.

A preferred future prospective clinical trial would involve examining the efficacy and safety of DM in combination with quinidine in patients who will have met the DSM-V diagnostic criteria for MDD and will be considered treatment-resistant. It is unknown if DM/quinidine will make MDD patients feel better, have no effect, or even worsen their condition, thus patients who are treatment-resistant as well as physicians overseeing these patients would be more inclined to test this innovative treatment option compared to newly diagnosed MDD patients. Moreover, if DM/quinidine is effective, it is likely to be "true" effect (vs. placebo effect) because the study participants have already tried and remain unresponsive to multiple current medication options. Existing medications that the subjects are taking will not serve as exclusion criteria unless they have known drug-drug interactions with the study medications, or if the doses will be significantly altered during the course of the study; since the patients are treatmentresistant, as long as their current medications remain constant throughout the study and do not interact with the study drugs, they should not affect the outcome. The trial will run for at least 6 weeks (considered to be an adequate trial with conventional antidepressants) with a return visit after the first 2-3 days and then at more spaced out intervals (e.g., at 2-3 days, week 1, week 2, week 4, and week 6) to ensure that rapid effects, if any, previously seen in the retrospective study in bipolar depression (Kelly and Lieberman, 2014) can be captured here in this study with MDD.

With the results presented in this Ph.D. thesis showing that DM produces antidepressant-like effects through two mechanisms that are distinct from modulation of the monoaminergic system and that may contribute to a faster onset of antidepressant effects, and the recent clinical evidence of antidepressant efficacy in treatment-resistant bipolar depression within a couple of days, it is likely that, for MDD, DM will produce therapeutic effects faster than conventional antidepressants and have efficacy in treatment-refractory cases. Future prospective clinical trials to test the efficacy of DM in MDD are warranted; because in spite of widespread antidepressant development since the 1950s, most of currently approved antidepressants are monoaminergic based and far from the ideal ones, resulting in undesirable 
side effects, requiring weeks to months of treatment to produce therapeutic effect, and remaining ineffective in about a third of the patients. Successful repurposing of DM as a fast, safe, and effective treatment for use in the general clinical population has the potential to transform the treatment of depression. 
Appendices 


\section{$\underline{\text { Appendix A }}$}

Materials and methods

\section{Animals}

Male, Swiss Webster mice (24-28 g; Harlan, Frederick, MD) were housed with food and water ad libitum, with a 12:12 h light-dark cycle. Animals were housed in groups of five for at least one week prior to initiation of experiments. All procedures were conducted in strict accordance with the recommendations in the Guide for the Care and Use of Laboratory Animals of the National Institutes of Health. The protocol was approved by the Institutional Animal Care and Use Committee at West Virginia University (Morgantown, WV), and all efforts were made to minimize suffering. Each experimental group consisted of 5-15 animals. The behavioral experiments (Figure 29) were carried out between 10:00 a.m. and 4:00 p.m. For a selected cohort, animals were killed by decapitation and the hippocampus and frontal cortex were extracted for western blot analysis ( $\mathrm{N}=8-10$ per group).

\section{Drugs and chemicals}

DM hydrobromide, d6-DM and quinidine sulfate were provided by Avanir Pharmaceuticals, Inc. (Aliso Viejo, CA) or purchased from Sigma-Aldrich (St. Louis, MO). Ketamine hydrochloride and imipramine hydrochloride were purchased from Sigma-Aldrich. Fluoxetine hydrochloride, BD1063 (1-[2(3,4-dichlorophenyl)ethyl]-4-methylpiperazine dihydrochloride), BD1047 (N-[2-(3,4dichlorophenyl)ethyl]-N-methyl-2-(dimethylamino)ethylamine dihydrobromide), and NBQX (2,3-dioxo6-nitro-1,2,3,4-tetrahydrobenzo[f]quinoxaline-7-sulfonamide disodium salt) were obtained from Tocris (Ellisville, MO). For the behavioral studies, all compounds were dissolved in sterile saline (Teknova, Hollister, CA). Mice received intraperitoneal (i.p.) injections of saline or drug solutions at a volume of 10 $\mathrm{ml} / \mathrm{kg}$ of body weight after acclimating to the open field test, 30 min prior to the TST or FST. Pretreatment with BD1063, BD1047 or NQBX was administered 15 min prior to the second drug (imipramine, ketamine or DM). For the saturation binding assays, $\left[{ }^{3} \mathrm{H}\right](+)$-pentazocine $(34.8 \mathrm{Ci} / \mathrm{mmol})$ 
Figure 29. Illustrations of FST, TST, and OFT.

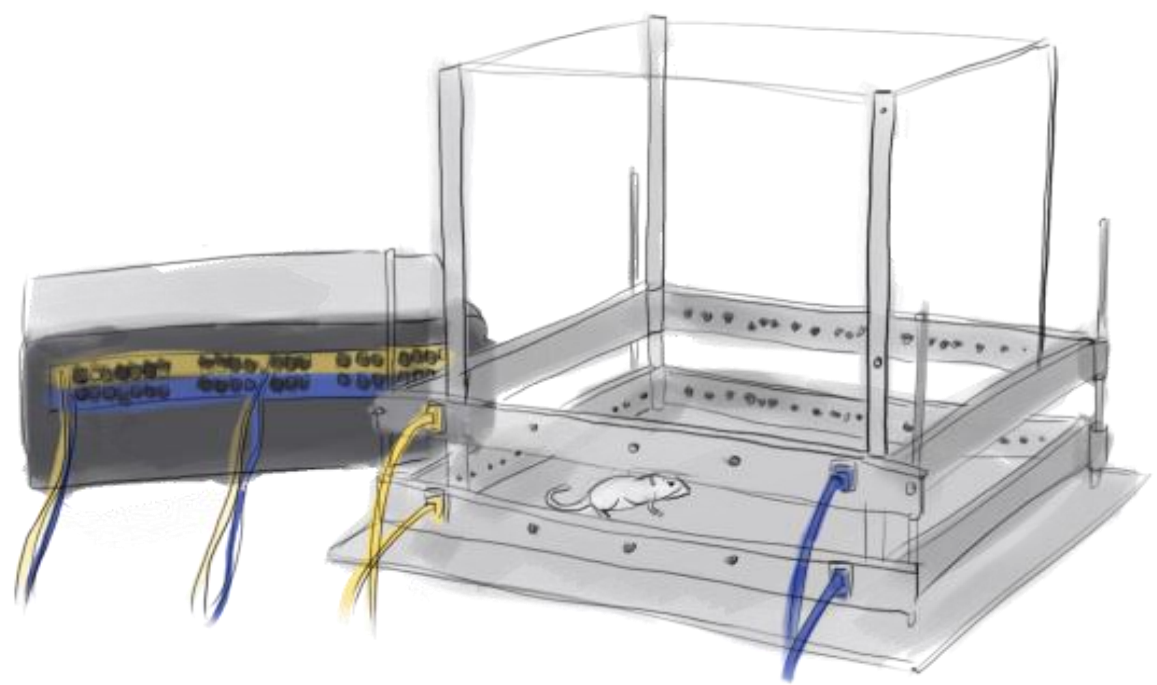

Open field test (OFT)

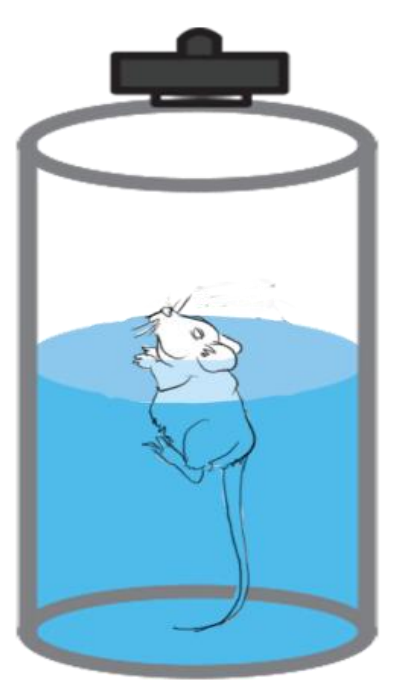

Forced swim test (FST)

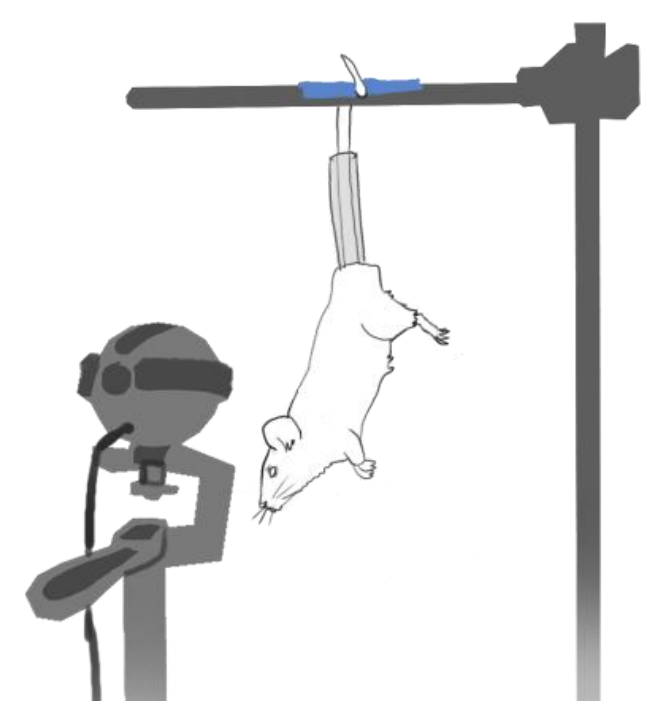

Tail suspension test (TST) 
was procured from Perkin Elmer (Hopkington, MA). All other chemicals and reagents were purchased from standard commercial suppliers (Sigma-Aldrich, St. Louis, MO).

\section{Open field test (OFT)}

Locomotor activity was measured utilizing an automated activity monitoring system (San Diego Instruments, San Diego, CA). Prior to locomotor activity measurements, animals were acclimated to the testing facility for at least $30 \mathrm{~min}$ and habituated to the testing chambers for an additional $30 \mathrm{~min}$. Each testing chamber consisted of a Plexiglas housing and a $16 \times 16$ photobeam array to detect lateral (ambulatory and fine) movements, with a separate 16 photobeam array to detect rearing activity. Subsequent to the acclimation period, animals were treated and placed back in their respective chambers. Ambulatory, fine and rearing movements were quantified and summated as a measure of total locomotor activity for the next $30 \mathrm{~min}$. Immediately after the locomotor measurements, mice were evaluated in either the FST or TST.

\section{Forced swim test (FST)}

Animals were placed in individual cylinders of water $(10 \mathrm{~cm}$ deep $)$ for a total of $6 \mathrm{~min}$ for the FST. The initial 2 min was an acclimation period and not scored. During the remaining 4 min, immobility time was quantified using ANY-Maze Version 4.63 video tracking software (Stoelting Co., Wood Dale, IL). Immobility was defined as no activity other than that required to maintain the animal's head above the surface of the water. ANY-Maze software settings were as follows: accustomization period = $120 \mathrm{~s}$, test duration $=240 \mathrm{~s}$, minimum immobility time $=2000 \mathrm{~ms}(2 \mathrm{~s})$, and immobility sensitivity $=65 \%$.

\section{Tail suspension test (TST)}

The TST was carried out according to the method described by Steru et al. (Alt et al., 2006), with modifications. To prevent tail climbing behaviors, a clear hollow polyvinyl chloride cylinder (Nalgene), cut to $4 \mathrm{~cm}$ length, was placed around the tail of each mouse (Alt et al., 2005). Each mouse was then hung 
upside down by the tail and fastened with adhesive tape $(2-4 \mathrm{~cm}$ from the tip of the tail) to a metal rod attached to a ring-stand. Mice were suspended $35 \mathrm{~cm}$ above the surface of a table, with the animals at least $15 \mathrm{~cm}$ from any object. Individual mice were videotaped using ANY-maze version 4.63 video tracking software for a total of $6 \mathrm{~min}$. The initial $2 \mathrm{~min}$ was an acclimation period and not scored. During the remaining $4 \mathrm{~min}$, immobility time was quantified by three observers blind to the experimental conditions. Immobility was defined as when the mice hung passively and completely motionless.

\section{Saturation binding assays}

To determine $\mathrm{K}_{\mathrm{d}}$ and $\mathrm{B}_{\max }$ by saturation binding, assays were performed in the absence (control) and presence of DM (400 nM) using methods previously published in detail (Nguyen et al., 2005). The concentration of DM used in these assays was based on the reported $\mathrm{K}_{\mathrm{i}}$ of the drug for sigma-1 receptors (Fishback et al., 2012). Briefly, 15 concentrations of $\left[{ }^{3} \mathrm{H}\right](+)$-pentazocine $(0.1-100 \mathrm{nM})$ were used to label sigma-1 receptors in $\mathrm{P}_{2}$ rat brain homogenates (400-500 $\left.\mu \mathrm{g} / \mathrm{sample}\right)$. Non-specific binding was determined in the presence of $10 \mu \mathrm{M}$ haloperidol. After a $120 \mathrm{~min}$ incubation at $25{ }^{\circ} \mathrm{C}$, the assays were terminated with the addition of $5 \mathrm{~mL}$ ice-cold $10 \mathrm{mM}$ Tris $\mathrm{HCl}, \mathrm{pH} 8.0$ and vacuum filtrated through glass fiber filters (Whatman GF/B, Brandel, Gaithersburg, MD) that were pre-soaked for at least $30 \mathrm{~min}$ with $1 \%$ polyethyleneimine (Sigma, St. Louis, MO). The filters were then washed twice with $5 \mathrm{~mL}$ icecold buffer, and the filter disks subsequently placed in Ecoscint cocktail (National Diagnostics, Manville, $\mathrm{NJ})$. Counts were extracted from the filters for at least $8 \mathrm{~h}$ prior to quantification with a scintillation counter (Beckman LS 6500, Beckman Instruments, Fullerton, CA).

\section{Western blot}

Immediately after completion of the behavioral measurements in the FST for a select cohort of mice, they were sacrificed by decapitation. Brains were then removed and bilateral hippocampus and frontal cortex samples dissected, flash frozen and stored at $-80^{\circ} \mathrm{C}$. Tissue was homogenized and lysed in radioimmunoprecipitation assay buffer (RIPA) buffer (Teknova, Hollister, CA) containing protease 
inhibitors (cOmplete ULTRA Protease Inhibitor Cocktail Tablets; Roche, Indianapolis, IN) and phosphatase inhibitors (phosSTOP Phosphatase Inhibitor Cocktail Tablets; Roche), and centrifuged at $13,000 \mathrm{~g}$ for $30 \mathrm{~min}$ at $4{ }^{\circ} \mathrm{C}$. The supernatant was assayed for total protein concentrations using bicinchoninic acid (BCA) protein assay kit (Pierce, Rockford, IL). Samples were run at 20 or $30 \mu \mathrm{g}$ of protein/well using Bolt Bis-Tris Plus 10\% pre-cast 15-well gels (Life Technologies, Grand Island, NY) in combination with 2 x Laemmli sample buffer (Bio-Rad, Hercules, CA). Gels were run using a Bolt Mini Gel system (Life Technologies) and in 2-(N-morpholino)ethane sulfonic acid (MES)-sodium dodecyl sulfate (SDS) Running Buffer (Life Technologies). After electrophoresis, gels were transferred to ImmunBlot Low Fluorescence polyvinylidene difluoride (PVDF) membranes (Bio-Rad) using a Mini Trans-Blot Electrophoretic Transfer Cell (Bio-Rad). Each tank was filled with Tris/glycine buffer containing 20\% (v/v) methanol, and transfers were carried out at $65 \mathrm{~V}$ for $2 \mathrm{~h}$ on ice. Blots were incubated with Odyssey blocking buffer (LI-COR Biosciences, Lincoln, NE) for $1 \mathrm{~h}$ at room temperature and then with the appropriate primary antibodies overnight at $4{ }^{\circ} \mathrm{C}$ (rabbit anti-pro-BDNF, 1:600, Alomone Labs, Jerusalem, Israel; rabbit anti-BDNF, 1:200, Alomone Labs; or mouse anti- $\alpha$-tubulin, 1:100,000, Cambridge, MA). After washing with Tris-buffered saline containing $0.1 \%$ Tween 20 , the blots were incubated with the secondary antibodies (IRDye 800CW goat anti-mouse or 700CW goat anti-rabbit, 1:10,000, LI-COR Biosciences) containing 0.01\% SDS for $45 \mathrm{~min}$ at room temperature. Visualization was carried out with an Odyssey Classic Infrared Imaging System (LI-COR Biosciences). Detected bands were quantified using Image Studio Lite Software (LI-COR Biosciences), normalized to relevant $\alpha$ tubulin values, and expressed as a percent of control density within individual blots.

\section{Neurite outgrowth cell culture}

Neurite outgrowth assays were performed as previously described with minor changes (Robson, 2012). Briefly, frozen PC12 cells purchased from American Type Culture Collection (Manassas, VA) were thawed and grown in complete media: Roswell Park Memorial Institute (RPMI) 1640 medium with $10 \%$ horse serum, $5 \%$ fetal bovine serum, and $0.5 \%$ penicillin/streptomycin, all purchased from 
Invitrogen (Carlsbad, CA). Cells were passaged by mechanical dissociation from culture flasks with Dulbecco's Phosphate-Buffered Saline (DPBS), and resuspended in RPMI 1640 medium with a minimal level $(0.5 \%)$ of fetal bovine serum at $1.5 \times 104$ cells $/ \mathrm{mL}$. A very low concentration of serum was used to minimize the endogenous sterols that might be present in the serum and can bind to sigma-1 receptors (Takebayashi et al., 2002). To find the optimal nerve growth factor (NGF) concentration for use in subsequent studies with drugs of interest, NGF (Alomone Labs, Jerusalem, Israel) was added to final concentrations of $0.039,0.078,0.156,0.3125,0.625,1.25,5.0$, and $10.0 \mathrm{ng} / \mathrm{mL}$. For the subsequent neurite outgrowth experiments, NGF was added to a final concentration of $0.3125 \mathrm{ng} / \mathrm{mL}$ with or without several drugs. Cells were plated at $1 \mathrm{~mL} /$ well in 24 well plates precoated with poly-D-lysine and laminin (Corning, Corning, NY) at $1 \mu \mathrm{g} / \mathrm{cm}^{2}$. Plates were then incubated at $37^{\circ} \mathrm{C}, 5 \% \mathrm{CO}_{2}$ for $48 \mathrm{~h}$.

\section{Quantification of neurite outgrowth}

Neurite outgrowth was estimated by using Integrated Modulation Contrast (IMC) microscopy (Leica DM IL, Leica Microsystems Inc., Buffalo Grove, IL) and was quantified as the percentage of cells bearing neurite processes of about 1 cell diameter or more in length. The magnification of the IMC microscopy was 10 -fold. Cells with neurites were counted approximately every 60 cells per field with three fields in each well. The field was selected from three separate areas (upper, middle, and bottom) in one well. At least three wells (i.e., average of $>180$ cells) were routinely used for each experimental condition. The counting was performed in a blinded manner. Cells in clusters were not included.

\section{Data analysis}

Data from all experiments were analyzed using GraphPad Prism 5.0 (San Diego, CA) by Student's unpaired t-test or one-way analysis of variance (ANOVA), followed when applicable by posthoc Dunnett's or Tukey's multiple comparison tests. The correlation between locomotor activity and immobility time was analyzed by Pearson's $\mathrm{r}$ correlation test. The $\mathrm{K}_{\mathrm{d}}$ and $\mathrm{B}_{\max }$ were determined using 
nonlinear regression and subsequently analyzed by unpaired t-tests. Data were represented as mean \pm SEM. $\mathrm{P}<0.05$ was considered statistically significant for all data analyzed. 


\title{
$\underline{\text { Appendix B }}$
}

\author{
References cited
}

Abdallah, C.G., Sanacora, G., Duman, R.S., and Krystal, J.H. (2015). Ketamine and rapid-acting antidepressants: a window into a new neurobiology for mood disorder therapeutics. Annual review of medicine 66, 509-523.

Adell, A., Castro, E., Celada, P., Bortolozzi, A., Pazos, A., and Artigas, F. (2005). Strategies for producing faster acting antidepressants. Drug discovery today 10, 578-585.

Akinfiresoye, L., and Tizabi, Y. (2013). Antidepressant effects of AMPA and ketamine combination: role of hippocampal BDNF, synapsin, and mTOR. Psychopharmacology 230, 291-298.

Alt, A., Nisenbaum, E.S., Bleakman, D., and Witkin, J.M. (2006). A role for AMPA receptors in mood disorders. Biochemical pharmacology 71, 1273-1288.

Alt, A., Witkin, J.M., and Bleakman, D. (2005). AMPA receptor potentiators as novel antidepressants. Current pharmaceutical design 11, 1511-1527.

American Psychiatric, A., American Psychiatric, A., and Force, D.S.M.T. (2013). Diagnostic and statistical manual of mental disorders : DSM-5.

Andersen, P.H. (1989). The dopamine inhibitor GBR 12909: selectivity and molecular mechanism of action. Eur J Pharmacol 166, 493-504.

Antoniou, T., and Juurlink, D.N. (2014). Dextromethorphan abuse. CMAJ : Canadian Medical Association journal = journal de l'Association medicale canadienne 186, E631.

Arregui, C.O., Carbonetto, S., and McKerracher, L. (1994). Characterization of neural cell adhesion sites: point contacts are the sites of interaction between integrins and the cytoskeleton in PC12 cells. The Journal of neuroscience : the official journal of the Society for Neuroscience 14, 6967-6977.

Association, A.P. (2008). The Practice of Electroconvulsive Therapy: Recommendations for Treatment, Training, and Privileging (A Task Force Report of the American Psychiatric Association) (American Psychiatric Publishing).

Autry, A.E., Adachi, M., Nosyreva, E., Na, E.S., Los, M.F., Cheng, P.F., Kavalali, E.T., and Monteggia, L.M. (2011). NMDA receptor blockade at rest triggers rapid behavioural antidepressant responses. Nature $475,91-95$.

Bai, F., Li, X., Clay, M., Lindstrom, T., and Skolnick, P. (2001). Intra- and interstrain differences in models of "behavioral despair". Pharmacol Biochem Behav 70, 187-192.

Bane, A., Rojas, D., Indermaur, K., Bennett, T., and Avery, D. (1996). Adverse effects of dextromethorphan on the spatial learning of rats in the Morris water maze. Eur J Pharmacol 302, 7-12.

Banken, J.A., and Foster, H. (2008). Dextromethorphan. Ann N Y Acad Sci 1139, 402-411.

Banker, G., and Goslin, K. (1998). Culturing Nerve Cells (MIT Press).

Barbon, A., Caracciolo, L., Orlandi, C., Musazzi, L., Mallei, A., La Via, L., Bonini, D., Mora, C., Tardito, D., Gennarelli, M., et al. (2011). Chronic antidepressant treatments induce a time-dependent upregulation of AMPA receptor subunit protein levels. Neurochem Int 59, 896-905.

Barbon, A., Popoli, M., La Via, L., Moraschi, S., Vallini, I., Tardito, D., Tiraboschi, E., Musazzi, L., Giambelli, R., Gennarelli, M., et al. (2006). Regulation of editing and expression of glutamate alphaamino-propionic-acid (AMPA)/kainate receptors by antidepressant drugs. Biol Psychiatry 59, 713-720. Belmaker, R.H. (2004). Bipolar Disorder. New England Journal of Medicine 351, 476-486.

Bem, J., and Peck, R. (1992). Dextromethorphan. Drug-Safety 7, 190-199.

Berlim, M.T., and Turecki, G. (2007). Definition, assessment, and staging of treatment-resistant refractory major depression: a review of current concepts and methods. Canadian journal of psychiatry Revue canadienne de psychiatrie 52, 46-54.

Bermack, J., Lavoie, N., Dryver, E., and Debonnel, G. (2002). Effects of sigma ligands on NMDA receptor function in the bulbectomy model of depression: a behavioural study in the rat. The international journal of neuropsychopharmacology / official scientific journal of the Collegium Internationale Neuropsychopharmacologicum 5, 53-62. 
Bermack, J.E., and Debonnel, G. (2001). Modulation of serotonergic neurotransmission by short- and long-term treatments with sigma ligands. Br J Pharmacol 134, 691-699.

Berman, R.M., Cappiello, A., Anand, A., Oren, D.A., Heninger, G.R., Charney, D.S., and Krystal, J.H. (2000). Antidepressant effects of ketamine in depressed patients. Biol Psychiatry 47, 351-354.

Blake, M.J., Gaedigk, A., Pearce, R.E., Bomgaars, L.R., Christensen, M.L., Stowe, C., James, L.P., Wilson, J.T., Kearns, G.L., and Leeder, J.S. (2007). Ontogeny of dextromethorphan O- and Ndemethylation in the first year of life. Clin Pharmacol Ther 81, 510-516.

Bleakman, D., Alt, A., and Witkin, J.M. (2007). AMPA receptors in the therapeutic management of depression. CNS \& neurological disorders drug targets 6, 117-126.

Bliss, T.V.P., and Cooke, S.F. (2011). Long-term potentiation and long-term depression: a clinical perspective. Clinics 66, 3-17.

Bradshaw, R.A., Hogue-Angeletti, R.A., and Frazier, W.A. (1974). Nerve growth factor and insulin: evidence of similarities in structure, function, and mechanism of action. Recent progress in hormone research 30, 575-596.

Bryner, J.K., Wang, U.K., Hui, J.W., Bedodo, M., MacDougall, C., and Anderson, I.B. (2006).

Dextromethorphan abuse in adolescence: an increasing trend: 1999-2004. Archives of pediatrics \& adolescent medicine 160, 1217-1222.

Burns, J.M., and Boyer, E.W. (2013). Antitussives and substance abuse. Substance abuse and rehabilitation 4, 75-82.

Bymaster, F.P., Katner, J.S., Nelson, D.L., Hemrick-Luecke, S.K., Threlkeld, P.G., Heiligenstein, J.H., Morin, S.M., Gehlert, D.R., and Perry, K.W. (2002). Atomoxetine increases extracellular levels of norepinephrine and dopamine in prefrontal cortex of rat: a potential mechanism for efficacy in attention deficit/hyperactivity disorder. Neuropsychopharmacology : official publication of the American College of Neuropsychopharmacology 27, 699-711.

Capon, D.A., Bochner, F., Kerry, N., Mikus, G., Danz, C., and Somogyi, A.A. (1996). The influence of CYP2D6 polymorphism and quinidine on the disposition and antitussive effect of dextromethorphan in humans. Clin Pharmacol Ther 60, 295-307.

Carpenter, C.L., Marks, S.S., Watson, D.L., and Greenberg, D.A. (1988). Dextromethorphan and dextrorphan as calcium channel antagonists. Brain Res 439, 372-375.

Carter, L.P., Reissig, C.J., Johnson, M.W., Klinedinst, M.A., Griffiths, R.R., and Mintzer, M.Z. (2013). Acute cognitive effects of high doses of dextromethorphan relative to triazolam in humans. Drug and alcohol dependence 128, 206-213.

Castren, E., and Rantamaki, T. (2010). The role of BDNF and its receptors in depression and antidepressant drug action: Reactivation of developmental plasticity. Developmental neurobiology 70 , 289-297.

Chen, B., Dowlatshahi, D., MacQueen, G.M., Wang, J.F., and Young, L.T. (2001). Increased hippocampal BDNF immunoreactivity in subjects treated with antidepressant medication. Biol Psychiatry 50, 260-265.

Chen, S.L., Lee, S.Y., Chang, Y.H., Chen, P.S., Lee, I.H., Wang, T.Y., Chen, K.C., Yang, Y.K., Hong, J.S., and Lu, R.B. (2014). Therapeutic effects of add-on low-dose dextromethorphan plus valproic acid in bipolar disorder. Eur Neuropsychopharmacol 24, 1753-1759.

Chilukuri, H., Reddy, N.P., Pathapati, R.M., Manu, A.N., Jollu, S., and Shaik, A.B. (2014). Acute antidepressant effects of intramuscular versus intravenous ketamine. Indian journal of psychological medicine 36, 71-76.

Chou, Y.C., Liao, J.F., Chang, W.Y., Lin, M.F., and Chen, C.F. (1999). Binding of dimemorfan to sigma1 receptor and its anticonvulsant and locomotor effects in mice, compared with dextromethorphan and dextrorphan. Brain Res 821, 516-519.

Church, J., Jones, M.G., Davies, S.N., and Lodge, D. (1989). Antitussive agents as N-methylaspartate antagonists: further studies. Canadian journal of physiology and pharmacology 67, 561-567.

Church, J., Lodge, D., and Berry, S.C. (1985). Differential effects of dextrorphan and levorphanol on the excitation of rat spinal neurons by amino acids. Eur J Pharmacol 111, 185-190. 
Cobos, E.J., Baeyens, J.M., and Del Pozo, E. (2005). Phenytoin differentially modulates the affinity of agonist and antagonist ligands for sigma 1 receptors of guinea pig brain. Synapse 55, 192-195.

Codd, E.E., Shank, R.P., Schupsky, J.J., and Raffa, R.B. (1995). Serotonin and norepinephrine uptake inhibiting activity of centrally acting analgesics: structural determinants and role in antinociception. $\mathrm{J}$ Pharmacol Exp Ther 274, 1263-1270.

Croom, K.F., Perry, C.M., and Plosker, G.L. (2009). Mirtazapine: a review of its use in major depression and other psychiatric disorders. CNS Drugs 23, 427-452.

Cryan, J.F., and Holmes, A. (2005). The ascent of mouse: advances in modelling human depression and anxiety. Nature reviews Drug discovery 4, 775-790.

Cryan, J.F., Markou, A., and Lucki, I. (2002). Assessing antidepressant activity in rodents: recent developments and future needs. Trends Pharmacol Sci 23, 238-245.

Cryan, J.F., Mombereau, C., and Vassout, A. (2005). The tail suspension test as a model for assessing antidepressant activity: review of pharmacological and genetic studies in mice. Neuroscience and biobehavioral reviews 29, 571-625.

Cusack, B., Nelson, A., and Richelson, E. (1994). Binding of antidepressants to human brain receptors: focus on newer generation compounds. Psychopharmacology 114, 559-565.

Cusin, C., Hilton, G.Q., Nierenberg, A.A., and Fava, M. (2012). Long-term maintenance with intramuscular ketamine for treatment-resistant bipolar II depression. The American journal of psychiatry 169, 868-869.

Damaj, M.I., Flood, P., Ho, K.K., May, E.L., and Martin, B.R. (2005). Effect of dextrometorphan and dextrorphan on nicotine and neuronal nicotinic receptors: in vitro and in vivo selectivity. J Pharmacol Exp Ther 312, 780-785.

Dematteis, M., Lallement, G., and Mallaret, M. (1998). Dextromethorphan and dextrorphan in rats: common antitussives--different behavioural profiles. Fundam Clin Pharmacol 12, 526-537.

Diamond, P.R., Farmery, A.D., Atkinson, S., Haldar, J., Williams, N., Cowen, P.J., Geddes, J.R., and McShane, R. (2014). Ketamine infusions for treatment resistant depression: a series of 28 patients treated weekly or twice weekly in an ECT clinic. Journal of psychopharmacology 28, 536-544.

Diazgranados, N., Ibrahim, L., Brutsche, N.E., Newberg, A., Kronstein, P., Khalife, S., Kammerer, W.A., Quezado, Z., Luckenbaugh, D.A., Salvadore, G., et al. (2010a). A randomized add-on trial of an Nmethyl-D-aspartate antagonist in treatment-resistant bipolar depression. Arch Gen Psychiatry 67, 793802.

DiazGranados, N., Ibrahim, L.A., Brutsche, N.E., Ameli, R., Henter, I.D., Luckenbaugh, D.A., MachadoVieira, R., and Zarate, C.A., Jr. (2010b). Rapid resolution of suicidal ideation after a single infusion of an $\mathrm{N}$-methyl-D-aspartate antagonist in patients with treatment-resistant major depressive disorder. The Journal of clinical psychiatry 71, 1605-1611.

Dicou, E., Rangon, C.M., Guimiot, F., Spedding, M., and Gressens, P. (2003). Positive allosteric modulators of AMPA receptors are neuroprotective against lesions induced by an NMDA agonist in neonatal mouse brain. Brain Res 970, 221-225.

Drevets, W.C., and Furey, M.L. (2010). Replication of scopolamine's antidepressant efficacy in major depressive disorder: a randomized, placebo-controlled clinical trial. Biol Psychiatry 67, 432-438.

Du, J., Suzuki, K., Wei, Y., Wang, Y., Blumenthal, R., Chen, Z., Falke, C., Zarate, C.A., Jr., and Manji, H.K. (2007). The anticonvulsants lamotrigine, riluzole, and valproate differentially regulate AMPA receptor membrane localization: relationship to clinical effects in mood disorders.

Neuropsychopharmacology : official publication of the American College of Neuropsychopharmacology 32, 793-802.

Duman, R.S. (2014a). Neurobiology of stress, depression, and rapid acting antidepressants: remodeling synaptic connections. Depression and anxiety 31, 291-296.

Duman, R.S. (2014b). Pathophysiology of depression and innovative treatments: remodeling glutamatergic synaptic connections. Dialogues in clinical neuroscience 16, 11-27.

Duman, R.S., and Monteggia, L.M. (2006). A neurotrophic model for stress-related mood disorders. Biol Psychiatry 59, 1116-1127. 
Duman, R.S., Tallman, J.F., and Nestler, E.J. (1988). Acute and chronic opiate-regulation of adenylate cyclase in brain: specific effects in locus coeruleus. J Pharmacol Exp Ther 246, 1033-1039.

Duman, R.S., and Voleti, B. (2012). Signaling pathways underlying the pathophysiology and treatment of depression: novel mechanisms for rapid-acting agents. Trends in neurosciences 35, 47-56.

Dwivedi, Y., Rizavi, H.S., Roberts, R.C., Conley, R.C., Tamminga, C.A., and Pandey, G.N. (2001).

Reduced activation and expression of ERK1/2 MAP kinase in the post-mortem brain of depressed suicide subjects. J Neurochem 77, 916-928.

Edward Roberts, R., Curran, H.V., Friston, K.J., and Morgan, C.J. (2014). Abnormalities in white matter microstructure associated with chronic ketamine use. Neuropsychopharmacology : official publication of the American College of Neuropsychopharmacology 39, 329-338.

Esteban, S., Lladó, J., Sastre-Coll, A., and García-Sevilla, J.A. (1999). Activation and desensitization by cyclic antidepressant drugs of $\alpha 2$-autoreceptors, $\alpha 2$-heteroreceptors and 5-HT1A-autoreceptors regulating monoamine synthesis in the rat brain in vivo. Naunyn-Schmiedeberg's Arch Pharmacol 360, 135-143.

Farley, S., Apazoglou, K., Witkin, J.M., Giros, B., and Tzavara, E.T. (2010). Antidepressant-like effects of an AMPA receptor potentiator under a chronic mild stress paradigm. The international journal of neuropsychopharmacology / official scientific journal of the Collegium Internationale Neuropsychopharmacologicum 13, 1207-1218.

Fava, M., and Kendler, K.S. (2000). Major Depressive Disorder. Neuron 28, 335-341.

Feder, A., Parides, M.K., Murrough, J.W., Perez, A.M., Morgan, J.E., Saxena, S., Kirkwood, K., Aan Het Rot, M., Lapidus, K.A., Wan, L.B., et al. (2014). Efficacy of intravenous ketamine for treatment of chronic posttraumatic stress disorder: a randomized clinical trial. JAMA psychiatry 71, 681-688.

Feyissa, A.M., Chandran, A., Stockmeier, C.A., and Karolewicz, B. (2009). Reduced levels of NR2A and NR2B subunits of NMDA receptor and PSD-95 in the prefrontal cortex in major depression. Progress in neuro-psychopharmacology \& biological psychiatry 33, 70-75.

Fishback, J.A., Robson, M.J., Xu, Y.T., and Matsumoto, R.R. (2010). Sigma receptors: potential targets for a new class of antidepressant drug. Pharmacol Ther 127, 271-282.

Fishback, J.A., Rosen, A., Bhat, R., McCurdy, C.R., and Matsumoto, R.R. (2012). A 96-well filtration method for radioligand binding analysis of sigma receptor ligands. J Pharm Biomed Anal 71, 157-161. Fleming, J.J., and England, P.M. (2010). Developing a complete pharmacology for AMPA receptors: A perspective on subtype-selective ligands. Bioorganic \& Medicinal Chemistry 18, 1381-1387.

Fleming, P.M. (1986). Dependence on dextromethorphan hydrobromide. Br Med J (Clin Res Ed) 293, 597.

Flynn, K.C., Pak, C.W., Shaw, A.E., Bradke, F., and Bamburg, J.R. (2009). Growth cone-like waves transport actin and promote axonogenesis and neurite branching. Developmental neurobiology 69, 761779.

Franklin, P.H., and Murray, T.F. (1992). High affinity [3H]dextrorphan binding in rat brain is localized to a noncompetitive antagonist site of the activated N-methyl-D-aspartate receptor-cation channel. Mol Pharmacol 41, 134-146.

Freudenberg, F., Celikel, T., and Reif, A. (2015). The role of $\alpha$-amino-3-hydroxy-5-methyl-4isoxazolepropionic acid (AMPA) receptors in depression: Central mediators of pathophysiology and antidepressant activity? Neuroscience \& Biobehavioral Reviews.

Fujimoto, M., Hayashi, T., Urfer, R., Mita, S., and Su, T.P. (2012). Sigma-1 receptor chaperones regulate the secretion of brain-derived neurotrophic factor. Synapse 66, 630-639.

Furey, M.L., and Drevets, W.C. (2006). Antidepressant efficacy of the antimuscarinic drug scopolamine: a randomized, placebo-controlled clinical trial. Arch Gen Psychiatry 63, 1121-1129.

Gant, T.G. (2014). Using deuterium in drug discovery: leaving the label in the drug. Journal of medicinal chemistry 57, 3595-3611.

Garcia, L.S., Comim, C.M., Valvassori, S.S., Reus, G.Z., Barbosa, L.M., Andreazza, A.C., Stertz, L., Fries, G.R., Gavioli, E.C., Kapczinski, F., et al. (2008). Acute administration of ketamine induces antidepressant-like effects in the forced swimming test and increases BDNF levels in the rat hippocampus. Progress in neuro-psychopharmacology \& biological psychiatry 32, 140-144. 
Garcia, L.S.B., Comim, C.M., Valvassori, S.S., Réus, G.Z., Stertz, L., Kapczinski, F., Gavioli, E.C., and Quevedo, J. (2009). Ketamine treatment reverses behavioral and physiological alterations induced by chronic mild stress in rats. Progress in Neuro-Psychopharmacology and Biological Psychiatry 33, 450455.

Gardier, A.M., Malagié, I., Trillat, A.C., Jacquot, C., and Artigas, F. (1996). Role of 5-HT1A autoreceptors in the mechanism of action of serotoninergic antidepressant drugs: recent findings from in vivo microdialysis studies. Fundamental \& Clinical Pharmacology 10, 16-27.

Ghasemi, M., Kazemi, M.H., Yoosefi, A., Ghasemi, A., Paragomi, P., Amini, H., and Afzali, M.H. (2014). Rapid antidepressant effects of repeated doses of ketamine compared with electroconvulsive therapy in hospitalized patients with major depressive disorder. Psychiatry research 215, 355-361. Gombos, Z., Spiller, A., Cottrell, G.A., Racine, R.J., and McIntyre Burnham, W. (1999). Mossy fiber sprouting induced by repeated electroconvulsive shock seizures. Brain Res 844, 28-33.

Grande, I., Fries, G.R., Kunz, M., and Kapczinski, F. (2010). The role of BDNF as a mediator of neuroplasticity in bipolar disorder. Psychiatry Investig 7, 243-250.

Greene, L.A., and Tischler, A.S. (1976). Establishment of a noradrenergic clonal line of rat adrenal pheochromocytoma cells which respond to nerve growth factor. Proceedings of the National Academy of Sciences of the United States of America 73, 2424-2428.

Greene, L.A., and Tischler, A.S. (1982). PC12 pheochromocytoma cultures in neurobiological research. Adv Cell Neurobiol 3, 373-414.

Guitart, X., Mendez, R., Ovalle, S., Andreu, F., Carceller, A., Farre, A.J., and Zamanillo, D. (2000). Regulation of ionotropic glutamate receptor subunits in different rat brain areas by a preferential sigma(1) receptor ligand and potential atypical antipsychotic. Neuropsychopharmacology : official publication of the American College of Neuropsychopharmacology 23, 539-546.

Haas, D.A., and Harper, D.G. (1992). Ketamine: a review of its pharmacologic properties and use in ambulatory anesthesia. Anesthesia progress 39, 61-68.

Harry, G.J., Billingsley, M., Bruinink, A., Campbell, I.L., Classen, W., Dorman, D.C., Galli, C., Ray, D., Smith, R.A., and Tilson, H.A. (1998). In vitro techniques for the assessment of neurotoxicity.

Environmental health perspectives $106 \mathrm{Suppl}$ 1, 131-158.

Hashimoto, K. (2011). The role of glutamate on the action of antidepressants. Progress in neuropsychopharmacology \& biological psychiatry 35, 1558-1568.

Hayashi, T., and Su, T.P. (2007). Sigma-1 receptor chaperones at the ER-mitochondrion interface regulate $\mathrm{Ca}(2+)$ signaling and cell survival. Cell 131, 596-610.

Hayashi, T., and Su, T.P. (2008). An update on the development of drugs for neuropsychiatric disorders: focusing on the sigma 1 receptor ligand. Expert Opin Ther Targets 12, 45-58.

Hernandez, S.C., Bertolino, M., Xiao, Y., Pringle, K.E., Caruso, F.S., and Kellar, K.J. (2000).

Dextromethorphan and its metabolite dextrorphan block alpha3beta4 neuronal nicotinic receptors. J

Pharmacol Exp Ther 293, 962-967.

Hinsberger, A., Sharma, V., and Mazmanian, D. (1994). Cognitive deterioration from long-term abuse of dextromethorphan: a case report. Journal of psychiatry \& neuroscience : JPN 19, 375-377.

Hollander, D., Pradas, J., Kaplan, R., McLeod, H.L., Evans, W.E., and Munsat, T.L. (1994). High-dose dextromethorphan in amyotrophic lateral sclerosis: phase I safety and pharmacokinetic studies. Annals of neurology 36, 920-924.

Holtzheimer, P.E., and Mayberg, H.S. (2011). Stuck in a rut: rethinking depression and its treatment. Trends in neurosciences 34, 1-9.

Husain, M.M., Rush, A.J., Fink, M., Knapp, R., Petrides, G., Rummans, T., Biggs, M.M., O'Connor, K., Rasmussen, K., Litle, M., et al. (2004). Speed of response and remission in major depressive disorder with acute electroconvulsive therapy (ECT): a Consortium for Research in ECT (CORE) report. The Journal of clinical psychiatry 65, 485-491.

Invernizzi, R.W., and Garattini, S. (2004). Role of presynaptic $\alpha 2$-adrenoceptors in antidepressant action: recent findings from microdialysis studies. Progress in Neuro-Psychopharmacology and Biological Psychiatry 28, 819-827. 
Ishima, T., Fujita, Y., and Hashimoto, K. (2014). Interaction of new antidepressants with sigma-1 receptor chaperones and their potentiation of neurite outgrowth in PC12 cells. Eur J Pharmacol 727, 167173.

Ishima, T., Nishimura, T., Iyo, M., and Hashimoto, K. (2008). Potentiation of nerve growth factorinduced neurite outgrowth in PC12 cells by donepezil: role of sigma-1 receptors and IP3 receptors. Progress in neuro-psychopharmacology \& biological psychiatry 32, 1656-1659.

Jeon, C.Y., Jin, J.K., Koh, Y.H., Chun, W., Choi, I.G., Kown, H.J., Kim, Y.S., and Park, J.B. (2010). Neurites from PC12 cells are connected to each other by synapse-like structures. Synapse 64, 765-772. Jin, L. (2007). Antagonists of AMPA-type receptor channels: A Patch Clamp Study. In Department of Neurology (Hannover: Medical School of Hannover).

Jourdi, H., Hsu, Y.T., Zhou, M., Qin, Q., Bi, X., and Baudry, M. (2009). Positive AMPA receptor modulation rapidly stimulates BDNF release and increases dendritic mRNA translation. The Journal of neuroscience : the official journal of the Society for Neuroscience 29,8688-8697.

Kamei, J., Mori, T., Igarashi, H., and Kasuya, Y. (1992). Serotonin release in nucleus of the solitary tract and its modulation by antitussive drugs. Research communications in chemical pathology and pharmacology 76, 371-374.

Kamel, I.R., Wendling, W.W., Chen, D., Wendling, K.S., Harakal, C., and Carlsson, C. (2008). Nmethyl-D-aspartate (NMDA) antagonists--S(+)-ketamine, dextrorphan, and dextromethorphan--act as calcium antagonists on bovine cerebral arteries. Journal of neurosurgical anesthesiology 20, 241-248. Karege, F., Bondolfi, G., Gervasoni, N., Schwald, M., Aubry, J.-M., and Bertschy, G. (2005). Low BrainDerived Neurotrophic Factor (BDNF) levels in serum of depressed patients probably results from lowered platelet BDNF release unrelated to platelet reactivity. Biological Psychiatry 57, 1068-1072.

Kelly, T.F., and Lieberman, D.Z. (2014). The utility of the combination of dextromethorphan and quinidine in the treatment of bipolar II and bipolar NOS. Journal of affective disorders 167, 333-335. Kemp, J.A., and McKernan, R.M. (2002). NMDA receptor pathways as drug targets. Nature neuroscience 5 Suppl, 1039-1042.

Kessler, R.C., Berglund, P., Demler, O., Jin, R., Koretz, D., Merikangas, K.R., Rush, A.J., Walters, E.E., and Wang, P.S. (2003). The epidemiology of major depressive disorder: results from the National Comorbidity Survey Replication (NCS-R). JAMA 289, 3095-3105.

Kim, H.C., Bing, G., Jhoo, W.K., Kim, W.K., Shin, E.J., Im, D.H., Kang, K.S., and Ko, K.H. (2003a). Metabolism to dextrorphan is not essential for dextromethorphan's anticonvulsant activity against kainate in mice. Life sciences 72, 769-783.

Kim, H.C., Ko, K.H., Kim, W.K., Shin, E.J., Kang, K.S., Shin, C.Y., and Jhoo, W.K. (2001). Effects of dextromethorphan on the seizures induced by kainate and the calcium channel agonist BAY k-8644: comparison with the effects of dextrorphan. Behav Brain Res 120,169-175.

Kim, H.C., Shin, C.Y., Seo, D.O., Jhoo, J.H., Jhoo, W.K., Kim, W.K., Shin, E.J., Lee, Y.H., Lee, P.H., and Ko, K.H. (2003b). New morphinan derivatives with negligible psychotropic effects attenuate convulsions induced by maximal electroshock in mice. Life sciences 72, 1883-1895.

Klouz, A., Sapena, R., Liu, J., Maurice, T., Tillement, J.P., Papadopoulos, V., and Morin, D. (2002). Evidence for sigma-1-like receptors in isolated rat liver mitochondrial membranes. Br J Pharmacol 135, 1607-1615.

Knapp, R.J., Goldenberg, R., Shuck, C., Cecil, A., Watkins, J., Miller, C., Crites, G., and Malatynska, E. (2002). Antidepressant activity of memory-enhancing drugs in the reduction of submissive behavior model. Eur J Pharmacol 440, 27-35.

Koike, H., Iijima, M., and Chaki, S. (2011). Involvement of AMPA receptor in both the rapid and sustained antidepressant-like effects of ketamine in animal models of depression. Behav Brain Res 224, 107-111.

Krishnan, V., and Nestler, E.J. (2008). The molecular neurobiology of depression. Nature 455, 894-902. Krug, M., Matthies, R., Wagner, M., and Brodemann, R. (1993). Non-opioid antitussives and methadone differentially influence hippocampal long-term potentiation in freely moving rats. Eur J Pharmacol 231, 355-361. 
Lamont, S.R., Paulls, A., and Stewart, C.A. (2001). Repeated electroconvulsive stimulation, but not antidepressant drugs, induces mossy fibre sprouting in the rat hippocampus. Brain Res 893, 53-58. Lapidus, K.A., Levitch, C.F., Perez, A.M., Brallier, J.W., Parides, M.K., Soleimani, L., Feder, A., Iosifescu, D.V., Charney, D.S., and Murrough, J.W. (2014). A randomized controlled trial of intranasal ketamine in major depressive disorder. Biol Psychiatry 76, 970-976.

Lara, D.R., Bisol, L.W., and Munari, L.R. (2013). Antidepressant, mood stabilizing and procognitive effects of very low dose sublingual ketamine in refractory unipolar and bipolar depression. The international journal of neuropsychopharmacology / official scientific journal of the Collegium Internationale Neuropsychopharmacologicum 16, 2111-2117.

Larkin, G.L., and Beautrais, A.L. (2011). A preliminary naturalistic study of low-dose ketamine for depression and suicide ideation in the emergency department. The international journal of neuropsychopharmacology / official scientific journal of the Collegium Internationale Neuropsychopharmacologicum 14, 1127-1131.

Lauterbach, E.C. (2012). An extension of hypotheses regarding rapid-acting, treatment-refractory, and conventional antidepressant activity of dextromethorphan and dextrorphan. Med Hypotheses 78, 693-702. Lauterborn, J.C., Lynch, G., Vanderklish, P., Arai, A., and Gall, C.M. (2000). Positive modulation of AMPA receptors increases neurotrophin expression by hippocampal and cortical neurons. The Journal of neuroscience : the official journal of the Society for Neuroscience 20, 8-21.

Lauterborn, J.C., Pineda, E., Chen, L.Y., Ramirez, E.A., Lynch, G., and Gall, C.M. (2009). Ampakines cause sustained increases in brain-derived neurotrophic factor signaling at excitatory synapses without changes in AMPA receptor subunit expression. Neuroscience 159, 283-295.

Lauterborn, J.C., Truong, G.S., Baudry, M., Bi, X., Lynch, G., and Gall, C.M. (2003). Chronic elevation of brain-derived neurotrophic factor by ampakines. J Pharmacol Exp Ther 307, 297-305.

Lee, J.H., Shin, E.J., Jeong, S.M., Kim, J.H., Lee, B.H., Yoon, I.S., Lee, J.H., Choi, S.H., Lee, S.M., Lee, P.H., et al. (2006). Effects of dextrorotatory morphinans on alpha3beta4 nicotinic acetylcholine receptors expressed in Xenopus oocytes. Eur J Pharmacol 536, 85-92.

Lee, S.Y., Chen, S.L., Chang, Y.H., Chen, S.H., Chu, C.H., Huang, S.Y., Tzeng, N.S., Wang, C.L., Lee, I.H., Yeh, T.L., et al. (2012). The DRD2/ANKK1 gene is associated with response to add-on dextromethorphan treatment in bipolar disorder. Journal of affective disorders 138, 295-300. Li, N., Lee, B., Liu, R.J., Banasr, M., Dwyer, J.M., Iwata, M., Li, X.Y., Aghajanian, G., and Duman, R.S. (2010). mTOR-dependent synapse formation underlies the rapid antidepressant effects of NMDA antagonists. Science 329, 959-964.

Li, X., Tizzano, J.P., Griffey, K., Clay, M., Lindstrom, T., and Skolnick, P. (2001). Antidepressant-like actions of an AMPA receptor potentiator (LY392098). Neuropharmacology 40, 1028-1033.

Li, X., Witkin, J.M., Need, A.B., and Skolnick, P. (2003). Enhancement of antidepressant potency by a potentiator of AMPA receptors. Cellular and molecular neurobiology 23, 419-430.

Liang, X., and Wang, R.Y. (1998). Biphasic modulatory action of the selective sigma receptor ligand SR $31742 \mathrm{~A}$ on N-methyl-D-aspartate-induced neuronal responses in the frontal cortex. Brain Res 807,208 213.

Liao, Y., Tang, J., Corlett, P.R., Wang, X., Yang, M., Chen, H., Liu, T., Chen, X., Hao, W., and Fletcher, P.C. (2011). Reduced dorsal prefrontal gray matter after chronic ketamine use. Biol Psychiatry 69, 42-48. Lindholm, J.S., Autio, H., Vesa, L., Antila, H., Lindemann, L., Hoener, M.C., Skolnick, P., Rantamaki, T., and Castren, E. (2012). The antidepressant-like effects of glutamatergic drugs ketamine and AMPA receptor potentiator LY 451646 are preserved in bdnf(+)/(-) heterozygous null mice. Neuropharmacology 62, 391-397.

Lu, B., Pang, P.T., and Woo, N.H. (2005). The yin and yang of neurotrophin action. Nat Rev Neurosci 6 , 603-614.

Lu, W.Y., and Bieger, D. (1996). Inhibition of nicotinic cholinoceptor mediated current in vagal motor neurons by local anesthetics. Canadian journal of physiology and pharmacology 74, 1265-1269.

Lucas, G., Rymar, V.V., Sadikot, A.F., and Debonnel, G. (2008). Further evidence for an antidepressant potential of the selective sigmal agonist SA 4503: electrophysiological, morphological and behavioural 
studies. The international journal of neuropsychopharmacology / official scientific journal of the Collegium Internationale Neuropsychopharmacologicum 11, 485-495.

Luckenbaugh, D.A., Niciu, M.J., Ionescu, D.F., Nolan, N.M., Richards, E.M., Brutsche, N.E., Guevara, S., and Zarate, C.A. (2014). Do the dissociative side effects of ketamine mediate its antidepressant effects? Journal of affective disorders 159, 56-61.

Lucki, I., Dalvi, A., and Mayorga, A.J. (2001). Sensitivity to the effects of pharmacologically selective antidepressants in different strains of mice. Psychopharmacology 155, 315-322.

Mackowiak, M., O'Neill, M.J., Hicks, C.A., Bleakman, D., and Skolnick, P. (2002). An AMPA receptor potentiator modulates hippocampal expression of BDNF: an in vivo study. Neuropharmacology 43, 1-10. Maeng, S., Zarate, C.A., Jr., Du, J., Schloesser, R.J., McCammon, J., Chen, G., and Manji, H.K. (2008). Cellular mechanisms underlying the antidepressant effects of ketamine: role of alpha-amino-3-hydroxy-5methylisoxazole-4-propionic acid receptors. Biol Psychiatry 63, 349-352.

Malatynska, E., Goldenberg, R., Shuck, L., Haque, A., Zamecki, P., Crites, G., Schindler, N., and Knapp, R.J. (2002). Reduction of submissive behavior in rats: a test for antidepressant drug activity.

Pharmacology 64, 8-17.

Malatynska, E., Rapp, R., Harrawood, D., and Tunnicliff, G. (2005). Submissive behavior in mice as a test for antidepressant drug activity. Pharmacol Biochem Behav 82, 306-313.

Malhotra, A.K., Pinals, D.A., Weingartner, H., Sirocco, K., Missar, C.D., Pickar, D., and Breier, A. (1996). NMDA receptor function and human cognition: the effects of ketamine in healthy volunteers. Neuropsychopharmacology : official publication of the American College of Neuropsychopharmacology 14, 301-307.

Markus, A., Patel, T.D., and Snider, W.D. (2002). Neurotrophic factors and axonal growth. Curr Opin Neurobiol 12, 523-531.

Martinez-Turrillas, R., Del Rio, J., and Frechilla, D. (2005). Sequential changes in BDNF mRNA expression and synaptic levels of AMPA receptor subunits in rat hippocampus after chronic antidepressant treatment. Neuropharmacology 49, 1178-1188.

Martinez-Turrillas, R., Del Rio, J., and Frechilla, D. (2007). Neuronal proteins involved in synaptic targeting of AMPA receptors in rat hippocampus by antidepressant drugs. Biochemical and biophysical research communications 353, 750-755.

Martinez-Turrillas, R., Frechilla, D., and Del Rio, J. (2002). Chronic antidepressant treatment increases the membrane expression of AMPA receptors in rat hippocampus. Neuropharmacology 43, 1230-1237. Matsumoto, R., Yu, Y., Mack, A., Coop, A., and Ash, J. (2005). Selective sigma receptor agonists produce antidepressant-like effects in mice. In Exp Biol, pp. 874.

Matsuno, K., Kobayashi, T., Tanaka, M.K., and Mita, S. (1996). Sigma 1 receptor subtype is involved in the relief of behavioral despair in the mouse forced swimming test. Eur J Pharmacol 312, 267-271. McArthur, R., and Borsini, F. (2006). Animal models of depression in drug discovery: a historical perspective. Pharmacol Biochem Behav 84, 436-452.

Messias, E., and Everett, B. (2012). Dextromethorphan and quinidine combination in emotional lability associated with depression: a case report. The primary care companion to CNS disorders 14 . Miller, A., Pratt, H., and Schiffer, R.B. (2011). Pseudobulbar affect: the spectrum of clinical presentations, etiologies and treatments. Expert review of neurotherapeutics 11, 1077-1088. Miller, S.C. (2011). Dextromethorphan to dextrorphan: a pathway towards abuse liability. Human psychopharmacology 26, 89-90; author reply 91.

Monteggia, L.M., and Zarate, C., Jr. (2015). Antidepressant actions of ketamine: from molecular mechanisms to clinical practice. Curr Opin Neurobiol 30C, 139-143.

Moret, C., and Briley, M. (2000). The possible role of 5-HT1B/D receptors in psychiatric disorders and their potential as a target for therapy. European Journal of Pharmacology 404, 1-12.

Morgan, C.J., Curran, H.V., and Independent Scientific Committee on, D. (2012a). Ketamine use: a review. Addiction 107, 27-38.

Morgan, C.J.A., Curran, H.V., and the Independent Scientific Committee on, D. (2012b). Ketamine use: a review. Addiction 107, 27-38. 
Moriguchi, S., Shinoda, Y., Yamamoto, Y., Sasaki, Y., Miyajima, K., Tagashira, H., and Fukunaga, K. (2013). Stimulation of the sigma-1 receptor by DHEA enhances synaptic efficacy and neurogenesis in the hippocampal dentate gyrus of olfactory bulbectomized mice. PloS one 8, e60863.

Mueller, T.I., and Leon, A.C. (1996). Recovery, chronicity, and levels of psychopathology in major depression. The Psychiatric clinics of North America 19, 85-102.

Muir, K.W., and Lees, K.R. (1995). Clinical Experience With Excitatory Amino Acid Antagonist Drugs. Stroke; a journal of cerebral circulation 26, 503-513.

Murrough, J.W., Iosifescu, D.V., Chang, L.C., Al Jurdi, R.K., Green, C.E., Perez, A.M., Iqbal, S., Pillemer, S., Foulkes, A., Shah, A., et al. (2013a). Antidepressant efficacy of ketamine in treatmentresistant major depression: a two-site randomized controlled trial. The American journal of psychiatry $170,1134-1142$.

Murrough, J.W., Wan, L.B., Iacoviello, B., Collins, K.A., Solon, C., Glicksberg, B., Perez, A.M., Mathew, S.J., Charney, D.S., Iosifescu, D.V., et al. (2013b). Neurocognitive effects of ketamine in treatment-resistant major depression: association with antidepressant response. Psychopharmacology.

Nagao, K., Kishi, T., Moriwaki, M., Fujita, K., Hirano, S., Yamanouchi, Y., Funahashi, T., and Iwata, N. (2013). Comparative clinical profile of mirtazapine and duloxetine in practical clinical settings in Japan: a 4-week open-label, parallel-group study of major depressive disorder. Neuropsychiatric disease and treatment 9, 781-786.

Nakamura, S. (1991). Axonal sprouting of noradrenergic locus coeruleus neurons following repeated stress and antidepressant treatment. Progress in brain research 88, 587-598.

Nam, Y., Shin, E.J., Yang, B.K., Bach, J.H., Jeong, J.H., Chung, Y.H., Park, E.S., Li, Z., Kim, K.W., Kwon, Y.B., et al. (2012). Dextromethorphan-induced psychotoxic behaviors cause sexual dysfunction in male mice via stimulation of sigma-1 receptors. Neurochem Int 61, 913-922.

Narita, N., Hashimoto, K., Tomitaka, S., and Minabe, Y. (1996). Interactions of selective serotonin reuptake inhibitors with subtypes of sigma receptors in rat brain. Eur J Pharmacol 307, 117-119. Nestler, E.J., Barrot, M., DiLeone, R.J., Eisch, A.J., Gold, S.J., and Monteggia, L.M. (2002a). Neurobiology of depression. Neuron 34, 13-25.

Nestler, E.J., Gould, E., Manji, H., Buncan, M., Duman, R.S., Greshenfeld, H.K., Hen, R., Koester, S., Lederhendler, I., Meaney, M., et al. (2002b). Preclinical models: status of basic research in depression. Biol Psychiatry 52, 503-528.

Netzer, R., Pflimlin, P., and Trube, G. (1993). Dextromethorphan blocks N-methyl-D-aspartate-induced currents and voltage-operated inward currents in cultured cortical neurons. Eur J Pharmacol 238, 209-216. Newcomer, J.W., Farber, N.B., Jevtovic-Todorovic, V., Selke, G., Melson, A.K., Hershey, T., Craft, S., and Olney, J.W. (1999). Ketamine-induced NMDA receptor hypofunction as a model of memory impairment and psychosis. Neuropsychopharmacology : official publication of the American College of Neuropsychopharmacology 20, 106-118.

Nguyen, E.C., McCracken, K.A., Liu, Y., Pouw, B., and Matsumoto, R.R. (2005). Involvement of sigma (sigma) receptors in the acute actions of methamphetamine: receptor binding and behavioral studies. Neuropharmacology 49, 638-645.

Nguyen, L., and Matsumoto, R.R. (2015). Involvement of AMPA receptors in the antidepressant-like effects of dextromethorphan in mice. Behav Brain Res.

Nguyen, L., Robson, M.J., Healy, J.R., Scandinaro, A.L., and Matsumoto, R.R. (2014). Involvement of sigma-1 receptors in the antidepressant-like effects of dextromethorphan. PloS one 9, e89985.

Niciu, M.J., Ionescu, D.F., Richards, E.M., and Zarate, C.A., Jr. (2013). Glutamate and its receptors in the pathophysiology and treatment of major depressive disorder. Journal of neural transmission.

NIMH (2015). In ClinicalTrialsgov [Internet] (Bethesda, MA: National Library of Medicine).

Nishimura, T., Ishima, T., Iyo, M., and Hashimoto, K. (2008). Potentiation of nerve growth factorinduced neurite outgrowth by fluvoxamine: role of sigma-1 receptors, IP3 receptors and cellular signaling pathways. PloS one 3, e2558.

Norrholm, S.D., and Ouimet, C.C. (2001). Altered dendritic spine density in animal models of depression and in response to antidepressant treatment. Synapse 42, 151-163. 
Olney, J.W., Labruyere, J., Wang, G., Wozniak, D.F., Price, M.T., and Sesma, M.A. (1991). NMDA antagonist neurotoxicity: mechanism and prevention. Science $254,1515-1518$.

Overstreet, D.H., Fredericks, K., Knapp, D., Breese, G., and McMichael, J. (2010). Nerve growth factor (NGF) has novel antidepressant-like properties in rats. Pharmacol Biochem Behav 94, 553-560.

Owens, M.J., Morgan, W.N., Plott, S.J., and Nemeroff, C.B. (1997). Neurotransmitter receptor and transporter binding profile of antidepressants and their metabolites. J Pharmacol Exp Ther 283, 13051322.

Pabba, M., Wong, A.Y., Ahlskog, N., Hristova, E., Biscaro, D., Nassrallah, W., Ngsee, J.K., Snyder, M., Beique, J.C., and Bergeron, R. (2014). NMDA receptors are upregulated and trafficked to the plasma membrane after sigma-1 receptor activation in the rat hippocampus. The Journal of neuroscience : the official journal of the Society for Neuroscience 34, 11325-11338.

Pacher, P., Kohegyi, E., Kecskemeti, V., and Furst, S. (2001). Current trends in the development of new antidepressants. Current medicinal chemistry 8, 89-100.

Palazidou, E. (2012). The neurobiology of depression. British medical bulletin 101, 127-145.

Palmer, G.C. (2001). Neuroprotection by NMDA receptor antagonists in a variety of neuropathologies. Current drug targets 2, 241-271.

Paslakis, G., Gilles, M., Meyer-Lindenberg, A., and Deuschle, M. (2010). Oral administration of the NMDA receptor antagonist S-ketamine as add-on therapy of depression: a case series.

Pharmacopsychiatry 43, 33-35.

Pattee, G.L., Wymer, J.P., Lomen-Hoerth, C., Appel, S.H., Formella, A.E., and Pope, L.E. (2014). An open-label multicenter study to assess the safety of dextromethorphan/quinidine in patients with pseudobulbar affect associated with a range of underlying neurological conditions. Current medical research and opinion 30, 2255-2265.

Paul, R.K., Singh, N.S., Khadeer, M., Moaddel, R., Sanghvi, M., Green, C.E., O’Loughlin, K., Torjman, M.C., Bernier, M., and Wainer, I.W. (2014). (R,S)-Ketamine Metabolites (R,S)-norketamine and (2S,6S)hydroxynorketamine Increase the Mammalian Target of Rapamycin Function. The Journal of the American Society of Anesthesiologists 121, 149-159.

Paveliev, M., Lume, M., Velthut, A., Phillips, M., Arumae, U., and Saarma, M. (2007). Neurotrophic factors switch between two signaling pathways that trigger axonal growth. Journal of cell science 120 , 2507-2516.

Perry, E.B., Jr., Cramer, J.A., Cho, H.S., Petrakis, I.L., Karper, L.P., Genovese, A., O'Donnell, E., Krystal, J.H., D'Souza, D.C., and Yale Ketamine Study, G. (2007). Psychiatric safety of ketamine in psychopharmacology research. Psychopharmacology 192, 253-260.

Pittenger, C., and Duman, R.S. (2007). Stress, Depression, and Neuroplasticity: A Convergence of Mechanisms. Neuropsychopharmacology : official publication of the American College of

Neuropsychopharmacology 33, 88-109.

Pope, L.E., Khalil, M.H., Berg, J.E., Stiles, M., Yakatan, G.J., and Sellers, E.M. (2004). Pharmacokinetics of dextromethorphan after single or multiple dosing in combination with quinidine in extensive and poor metabolizers. J Clin Pharmacol 44, 1132-1142.

Price, R.B., Nock, M.K., Charney, D.S., and Mathew, S.J. (2009). Effects of intravenous ketamine on explicit and implicit measures of suicidality in treatment-resistant depression. Biol Psychiatry 66, 522526.

Reissig, C.J., Carter, L.P., Johnson, M.W., Mintzer, M.Z., Klinedinst, M.A., and Griffiths, R.R. (2012). High doses of dextromethorphan, an NMDA antagonist, produce effects similar to classic hallucinogens. Psychopharmacology 223, 1-15.

Reus, G.Z., Stringari, R.B., Ribeiro, K.F., Ferraro, A.K., Vitto, M.F., Cesconetto, P., Souza, C.T., and Quevedo, J. (2011). Ketamine plus imipramine treatment induces antidepressant-like behavior and increases CREB and BDNF protein levels and PKA and PKC phosphorylation in rat brain. Behav Brain Res 221, 166-171. 
Reus, G.Z., Vieira, F.G., Abelaira, H.M., Michels, M., Tomaz, D.B., dos Santos, M.A., Carlessi, A.S., Neotti, M.V., Matias, B.I., Luz, J.R., et al. (2014). MAPK signaling correlates with the antidepressant effects of ketamine. Journal of psychiatric research 55, 15-21.

Robson, M.J., Elliott, M., Seminerio, M.J., and Matsumoto, R.R. (2012). Evaluation of sigma (sigma) receptors in the antidepressant-like effects of ketamine in vitro and in vivo. Eur Neuropsychopharmacol $22,308-317$.

Romanelli, F., and Smith, K.M. (2009). Dextromethorphan abuse: clinical effects and management. J Am Pharm Assoc (2003) 49, e20-25; quiz e26-27.

Ruf, B.M., and Bhagwagar, Z. (2009). The 5-HT1B receptor: a novel target for the pathophysiology of depression. Current drug targets 10,1118-1138.

Runyon, S.P., Savage, J.E., Taroua, M., Roth, B.L., Glennon, R.A., and Westkaemper, R.B. (2001). Influence of chain length and $\mathrm{N}$-alkylation on the selective serotonin receptor ligand 9-(aminomethyl)9,10-dihydroanthracene. Bioorganic \& medicinal chemistry letters 11, 655-658.

Rutter, J.J., Gundlah, C., and Auerbach, S.B. (1994). Increase in extracellular serotonin produced by uptake inhibitors is enhanced after chronic treatment with fluoxetine. Neuroscience letters 171, 183-186. Sabino, V., Cottone, P., Parylak, S.L., Steardo, L., and Zorrilla, E.P. (2009). Sigma-1 receptor knockout mice display a depressive-like phenotype. Behav Brain Res 198, 472-476.

Sackeim, H.A., Prudic, J., Fuller, R., Keilp, J., Lavori, P.W., and Olfson, M. (2007). The cognitive effects of electroconvulsive therapy in community settings. Neuropsychopharmacology : official publication of the American College of Neuropsychopharmacology 32, 244-254.

Santarelli, L., Saxe, M., Gross, C., Surget, A., Battaglia, F., Dulawa, S., Weisstaub, N., Lee, J., Duman, R., Arancio, O., et al. (2003). Requirement of hippocampal neurogenesis for the behavioral effects of antidepressants. Science 301, 805-809.

Schlaepfer, T.E., Agren, H., Monteleone, P., Gasto, C., Pitchot, W., Rouillon, F., Nutt, D.J., and Kasper, S. (2012). The hidden third: improving outcome in treatment-resistant depression. Journal of psychopharmacology 26, 587-602.

Schmid, B., Bircher, J., Preisig, R., and Kupfer, A. (1985). Polymorphic dextromethorphan metabolism: co-segregation of oxidative O-demethylation with debrisoquin hydroxylation. Clin Pharmacol Ther 38, 618-624.

Schmidt, H.D., and Duman, R.S. (2010). Peripheral BDNF Produces Antidepressant-Like Effects in Cellular and Behavioral Models. Neuropsychopharmacology : official publication of the American College of Neuropsychopharmacology 35, 2378-2391.

Schobel, S.A., Chaudhury, N.H., Khan, U.A., Paniagua, B., Styner, M.A., Asllani, I., Inbar, B.P., Corcoran, C.M., Lieberman, J.A., Moore, H., et al. (2013). Imaging patients with psychosis and a mouse model establishes a spreading pattern of hippocampal dysfunction and implicates glutamate as a driver. Neuron 78, 81-93.

Schoedel, K.A., Morrow, S.A., and Sellers, E.M. (2014). Evaluating the safety and efficacy of dextromethorphan/quinidine in the treatment of pseudobulbar affect. Neuropsychiatric disease and treatment 10,1161-1174.

Schoedel, K.A., Pope, L.E., and Sellers, E.M. (2012). Randomized open-label drug-drug interaction trial of dextromethorphan/quinidine and paroxetine in healthy volunteers. Clinical drug investigation 32, 157169.

Schwartz, R.H. (2005). Adolescent abuse of dextromethorphan. Clin Pediatr (Phila) 44, 565-568. Shapira, B., and Lerer, B. (1999). Speed of response to bilateral ECT: an examination of possible predictors in two controlled trials. The journal of ECT 15, 202-206.

Shimizu, H., Takebayashi, M., Tani, M., Tanaka, H., Yamagata, B., Kurosawa, K., Yamada, H., Hachisu, M., Hisaoka-Nakashima, K., Okada-Tsuchioka, M., et al. (2013). Sigma-1 receptor concentration in plasma of patients with late-life depression: a preliminary study. Neuropsychiatric disease and treatment $8,1867-1872$. 
Shin, E.J., Bach, J.H., Lee, S.Y., Kim, J.M., Lee, J., Hong, J.S., Nabeshima, T., and Kim, H.C. (2011). Neuropsychotoxic and neuroprotective potentials of dextromethorphan and its analogs. Journal of pharmacological sciences 116, 137-148.

Shin, E.J., Nah, S.Y., Chae, J.S., Bing, G., Shin, S.W., Yen, T.P., Baek, I.H., Kim, W.K., Maurice, T., Nabeshima, T., et al. (2007). Dextromethorphan attenuates trimethyltin-induced neurotoxicity via sigma1 receptor activation in rats. Neurochem Int 50, 791-799.

Shiroma, P.R., Albott, C.S., Johns, B., Thuras, P., Wels, J., and Lim, K.O. (2014). Neurocognitive performance and serial intravenous subanesthetic ketamine in treatment-resistant depression. The international journal of neuropsychopharmacology / official scientific journal of the Collegium Internationale Neuropsychopharmacologicum 17, 1805-1813.

Shytle, R.D., Silver, A.A., Lukas, R.J., Newman, M.B., Sheehan, D.V., and Sanberg, P.R. (2002). Nicotinic acetylcholine receptors as targets for antidepressants. Mol Psychiatry 7, 525-535.

Skuza, G. (2003). Potential antidepressant activity of sigma ligands. Pol J Pharmacol 55, 923-934. Skuza, G., and Rogoz, Z. (2002). A potential antidepressant activity of SA4503, a selective sigma 1 receptor agonist. Behav Pharmacol 13, 537-543.

Slattery, D.A., Hudson, A.L., and Nutt, D.J. (2004). Invited review: the evolution of antidepressant mechanisms. Fundamental \& Clinical Pharmacology 18, 1-21.

Slotkin, T., and Seidler, F. (2009). Transcriptional profiles reveal similarities and differences in the effects of developmental neurotoxicants on differentiation into neurotransmitter phenotypes in PC12 cells. Brain research bulletin 78, 211-225.

Sofroniew, M.V., Howe, C.L., and Mobley, W.C. (2001). Nerve growth factor signaling, neuroprotection, and neural repair. Annual review of neuroscience 24, 1217-1281.

Sos, P., Klirova, M., Novak, T., Kohutova, B., Horacek, J., and Palenicek, T. (2013). Relationship of ketamine's antidepressant and psychotomimetic effects in unipolar depression. Neuroendocrinol Lett 34, 101-107.

Sparatore, B., Patrone, M., Passalacqua, M., Pedrazzi, M., Pontremoli, S., and Melloni, E. (2000). Neuronal differentiation of PC12 cells involves changes in protein kinase C-theta distribution and molecular properties. Biochemical and biophysical research communications 275, 149-153.

Stahl, S.M. (2013a). Mechanism of action of dextromethorphan/quinidine:comparison with ketamine. CNS spectrums 18, 225-227.

Stahl, S.M. (2013b). Stahl's essential psychopharmacology : neuroscientific basis and practical application, 4th edn (Cambridge: Cambridge University Press).

Stanton, T., Bolden-Watson, C., Cusack, B., and Richelson, E. (1993). Antagonism of the five cloned human muscarinic cholinergic receptors expressed in CHO-K1 cells by antidepressants and antihistaminics. Biochemical pharmacology 45, 2352-2354.

Statistics, N.C.f.H. (2014). Special Feature on Prescription Drugs.

Su, T.P., Hayashi, T., Maurice, T., Buch, S., and Ruoho, A.E. (2010). The sigma-1 receptor chaperone as an inter-organelle signaling modulator. Trends Pharmacol Sci 31, 557-566.

Sugimoto, Y., Kajiwara, Y., Hirano, K., Yamada, S., Tagawa, N., Kobayashi, Y., Hotta, Y., and Yamada, J. (2008). Mouse strain differences in immobility and sensitivity to fluvoxamine and desipramine in the forced swimming test: analysis of serotonin and noradrenaline transporter binding. Eur J Pharmacol 592, 116-122.

Svenningsson, P., Tzavara, E.T., Witkin, J.M., Fienberg, A.A., Nomikos, G.G., and Greengard, P. (2002). Involvement of striatal and extrastriatal DARPP-32 in biochemical and behavioral effects of fluoxetine (Prozac). Proceedings of the National Academy of Sciences of the United States of America 99, 31823187.

Szekely, J.I., Sharpe, L.G., and Jaffe, J.H. (1991). Induction of phencyclidine-like behavior in rats by dextrorphan but not dextromethorphan. Pharmacol Biochem Behav 40, 381-386.

Takebayashi, M., Hayashi, T., and Su, T.P. (2002). Nerve growth factor-induced neurite sprouting in PC12 cells involves sigma-1 receptors: implications for antidepressants. J Pharmacol Exp Ther 303, 1227-1237. 
Takebayashi, M., Hisaoka, K., Maeda, N., Tsuchioka, M. (2007). Altered levels of whole blood sigma-1 receptor protein in patients with mood disorders. Program No. 608.6/HH19. Paper presented at: Society for Neuroscience (San Diego).

Takemura, R., Okabe, S., Kobayashi, N., and Hirokawa, N. (1993). Reorganization of brain spectrin (fodrin) during differentiation of PC12 cells. Neuroscience 52, 381-391.

Tatsumi, M., Groshan, K., Blakely, R.D., and Richelson, E. (1997). Pharmacological profile of antidepressants and related compounds at human monoamine transporters. Eur J Pharmacol 340, 249-258. Thase, M.E., Greenhouse, J.B., Frank, E., Reynolds, C.F., 3rd, Pilkonis, P.A., Hurley, K., Grochocinski, V., and Kupfer, D.J. (1997). Treatment of major depression with psychotherapy or psychotherapypharmacotherapy combinations. Arch Gen Psychiatry 54, 1009-1015.

Tischler, A.S., and Greene, L.A. (1975). Nerve growth factor-induced process formation by cultured rat pheochromocytoma cells. Nature 258, 341-342.

Tortella, F.C., Pellicano, M., and Bowery, N.G. (1989). Dextromethorphan and neuromodulation: old drug coughs up new activities. Trends Pharmacol Sci 10, 501-507.

Tran, V.T., Chang, R.S., and Snyder, S.H. (1978). Histamine H1 receptors identified in mammalian brain membranes with $[3 \mathrm{H}]$ mepyramine. Proceedings of the National Academy of Sciences of the United States of America 75, 6290-6294.

Trivedi, M.H., Rush, A.J., Wisniewski, S.R., Nierenberg, A.A., Warden, D., Ritz, L., Norquist, G., Howland, R.H., Lebowitz, B., McGrath, P.J., et al. (2006). Evaluation of outcomes with citalopram for depression using measurement-based care in STAR*D: implications for clinical practice. The American journal of psychiatry $163,28-40$.

Tsai, S.Y., Hayashi, T., Harvey, B.K., Wang, Y., Wu, W.W., Shen, R.F., Zhang, Y., Becker, K.G., Hoffer, B.J., and Su, T.P. (2009). Sigma-1 receptors regulate hippocampal dendritic spine formation via a free radical-sensitive mechanism involving Rac1xGTP pathway. Proceedings of the National Academy of Sciences of the United States of America 106, 22468-22473.

Ukai, M., Maeda, H., Nanya, Y., Kameyama, T., and Matsuno, K. (1998). Beneficial effects of acute and repeated administrations of sigma receptor agonists on behavioral despair in mice exposed to tail suspension. Pharmacol Biochem Behav 61, 247-252.

Urani, A., Roman, F.J., Phan, V.L., Su, T.P., and Maurice, T. (2001). The antidepressant-like effect induced by sigma(1)-receptor agonists and neuroactive steroids in mice submitted to the forced swimming test. J Pharmacol Exp Ther 298, 1269-1279.

Valenstein, M., Vijan, S., Zeber, J.E., Boehm, K., and Buttar, A. (2001). The cost-utility of screening for depression in primary care. Annals of internal medicine 134, 345-360.

Valentine, G.W., Mason, G.F., Gomez, R., Fasula, M., Watzl, J., Pittman, B., Krystal, J.H., and Sanacora, G. (2011). The antidepressant effect of ketamine is not associated with changes in occipital amino acid neurotransmitter content as measured by [(1)H]-MRS. Psychiatry research 191, 122-127.

Vaudry, D., Stork, P.J.S., Lazarovici, P., and Eiden, L.E. (2002). Signaling Pathways for PC12 Cell Differentiation: Making the Right Connections. Science 296, 1648-1649.

Vaugeois, J.M., Passera, G., Zuccaro, F., and Costentin, J. (1997). Individual differences in response to imipramine in the mouse tail suspension test. Psychopharmacology 134, 387-391.

Voleti, B., Navarria, A., Liu, R.J., Banasr, M., Li, N., Terwilliger, R., Sanacora, G., Eid, T., Aghajanian, G., and Duman, R.S. (2013). Scopolamine rapidly increases mammalian target of rapamycin complex 1 signaling, synaptogenesis, and antidepressant behavioral responses. Biol Psychiatry 74, 742-749.

Volz, H.P., and Stoll, K.D. (2004). Clinical trials with sigma ligands. Pharmacopsychiatry 37 Suppl 3, S214-220.

Wade, A.G., and Haring, J. (2010). A review of the costs associated with depression and treatment noncompliance: the potential benefits of online support. International clinical psychopharmacology 25 , 288-296.

Walker, J., and Yatham, L.N. (1993). Benylin (dextromethorphan) abuse and mania. Bmj 306, 896. 
Wan, L.B., Levitch, C.F., Perez, A.M., Brallier, J.W., Iosifescu, D.V., Chang, L.C., Foulkes, A., Mathew, S.J., Charney, D.S., and Murrough, J.W. (2014). Ketamine safety and tolerability in clinical trials for treatment-resistant depression. The Journal of clinical psychiatry.

Wang, C., Zheng, D., Xu, J., Lam, W., and Yew, D.T. (2013). Brain damages in ketamine addicts as revealed by magnetic resonance imaging. Frontiers in neuroanatomy 7, 23.

Wang, J., Mack, A.L., Coop, A., and Matsumoto, R.R. (2007). Novel sigma (sigma) receptor agonists produce antidepressant-like effects in mice. Eur Neuropsychopharmacol 17, 708-716.

Watanabe, N., Omori, I.M., Nakagawa, A., Cipriani, A., Barbui, C., Churchill, R., and Furukawa, T.A. (2011). Mirtazapine versus other antidepressive agents for depression. The Cochrane database of systematic reviews, CD006528.

Werling, L.L., Keller, A., Frank, J.G., and Nuwayhid, S.J. (2007a). A comparison of the binding profiles of dextromethorphan, memantine, fluoxetine and amitriptyline: treatment of involuntary emotional expression disorder. Exp Neurol 207, 248-257.

Werling, L.L., Lauterbach, E.C., and Calef, U. (2007b). Dextromethorphan as a potential neuroprotective agent with unique mechanisms of action. Neurologist 13, 272-293.

WHO (2012). Depression. Fact sheet No. 369.

Wilson, M.D., Ferguson, R.W., Mazer, M.E., and Litovitz, T.L. (2011). Monitoring trends in dextromethorphan abuse using the National Poison Data System: 2000-2010. Clinical toxicology 49, 409415.

Wolak, M., Siwek, A., Szewczyk, B., Poleszak, E., Pilc, A., Popik, P., and Nowak, G. (2013). Involvement of NMDA and AMPA receptors in the antidepressant-like activity of antidepressant drugs in the forced swim test. Pharmacological reports : PR 65, 991-997.

Wolf, N.J., and Hopko, D.R. (2008). Psychosocial and pharmacological interventions for depressed adults in primary care: A critical review. Clinical Psychology Review 28, 131-161.

Wolfe, T.R., and Caravati, E.M. (1995). Massive dextromethorphan ingestion and abuse. The American journal of emergency medicine 13, 174-176.

Wong, D.T., Threlkeld, P.G., Best, K.L., and Bymaster, F.P. (1982). A new inhibitor of norepinephrine uptake devoid of affinity for receptors in rat brain. J Pharmacol Exp Ther 222, 61-65.

Woodworth, J.R., Dennis, S.R., Moore, L., and Rotenberg, K.S. (1987). The polymorphic metabolism of dextromethorphan. J Clin Pharmacol 27, 139-143.

Wu, Z., and Bowen, W.D. (2008). Role of sigma-1 receptor C-terminal segment in inositol 1,4,5-

trisphosphate receptor activation: constitutive enhancement of calcium signaling in MCF-7 tumor cells. $\mathrm{J}$ Biol Chem 283, 28198-28215.

Yamamoto, H., Miura, R., Yamamoto, T., Shinohara, K., Watanabe, M., Okuyama, S., Nakazato, A., and Nukada, T. (1999). Amino acid residues in the transmembrane domain of the type 1 sigma receptor critical for ligand binding. FEBS Lett 445, 19-22.

Yamanaka, H., Yokoyama, C., Mizuma, H., Kurai, S., Finnema, S.J., Halldin, C., Doi, H., and Onoe, H. (2014). A possible mechanism of the nucleus accumbens and ventral pallidum 5-HT1B receptors underlying the antidepressant action of ketamine: a PET study with macaques. Translational psychiatry 4 , e342.

Yang, C., Hu, Y.M., Zhou, Z.Q., Zhang, G.F., and Yang, J.J. (2013). Acute administration of ketamine in rats increases hippocampal BDNF and mTOR levels during forced swimming test. Upsala journal of medical sciences 118, 3-8.

Yang, L.P., and Deeks, E.D. (2015). Dextromethorphan/Quinidine: a review of its use in adults with pseudobulbar affect. Drugs 75, 83-90.

Yu, A., and Haining, R.L. (2001). Comparative contribution to dextromethorphan metabolism by cytochrome P450 isoforms in vitro: can dextromethorphan be used as a dual probe for both CTP2D6 and CYP3A activities? Drug metabolism and disposition: the biological fate of chemicals 29, 1514-1520. Zanicotti, C.G., Perez, D., and Glue, P. (2012). Mood and pain responses to repeat dose intramuscular ketamine in a depressed patient with advanced cancer. Journal of palliative medicine 15, 400-403.c 
Zarate, C.A., Jr., Brutsche, N.E., Ibrahim, L., Franco-Chaves, J., Diazgranados, N., Cravchik, A., Selter, J., Marquardt, C.A., Liberty, V., and Luckenbaugh, D.A. (2012). Replication of ketamine's antidepressant efficacy in bipolar depression: a randomized controlled add-on trial. Biol Psychiatry 71, 939-946.

Zarate, C.A., Jr., Singh, J.B., Carlson, P.J., Brutsche, N.E., Ameli, R., Luckenbaugh, D.A., Charney, D.S., and Manji, H.K. (2006). A randomized trial of an N-methyl-D-aspartate antagonist in treatment-resistant major depression. Arch Gen Psychiatry 63, 856-864.

Zawertailo, L.A., Kaplan, H.L., Busto, U.E., Tyndale, R.F., and Sellers, E.M. (1998). Psychotropic effects of dextromethorphan are altered by the CYP2D6 polymorphism: a pilot study. Journal of clinical psychopharmacology 18, 332-337.

Zawertailo, L.A., Tyndale, R.F., Busto, U., and Sellers, E.M. (2010). Effect of metabolic blockade on the psychoactive effects of dextromethorphan. Human psychopharmacology 25, 71-79.

Zhang, T.Y., Cho, H.J., Lee, S., Lee, J.H., Choi, S.H., Ryu, V., Yoo, S.B., Lee, J.Y., Kim, D.G., and Jahng, J.W. (2007). Impairments in water maze learning of aged rats that received dextromethorphan repeatedly during adolescent period. Psychopharmacology 191, 171-179.

Zhang, X.J., Liu, L.L., Jiang, S.X., Zhong, Y.M., and Yang, X.L. (2011). Activation of the zeta receptor 1 suppresses NMDA responses in rat retinal ganglion cells. Neuroscience 177, 12-22.

Zhou, W., Wang, N., Yang, C., Li, X.M., Zhou, Z.Q., and Yang, J.J. (2013). Ketamine-induced antidepressant effects are associated with AMPA receptors-mediated upregulation of mTOR and BDNF in rat hippocampus and prefrontal cortex. European psychiatry : the journal of the Association of European Psychiatrists.

Zhou, W., Wang, N., Yang, C., Li, X.M., Zhou, Z.Q., and Yang, J.J. (2014). Ketamine-induced antidepressant effects are associated with AMPA receptors-mediated upregulation of mTOR and BDNF in rat hippocampus and prefrontal cortex. European psychiatry : the journal of the Association of European Psychiatrists 29, 419-423.

Zigman, D., and Blier, P. (2013). Urgent ketamine infusion rapidly eliminated suicidal ideation for a patient with major depressive disorder: a case report. Journal of clinical psychopharmacology 33, 270272. 


\author{
$\underline{\text { Appendix C }}$ \\ CV \\ LINDA NGUYEN \\ WVU School of Pharmacy - PO Box 9500 \\ 1 Medical Center Dr. \\ Morgantown, WV 26506-9500 \\ lnguyen1@mix.wvu.edu
}

\title{
EDUCATION
}

2011 - Present West Virginia University

M.D./Ph.D. Candidate - Pharmaceutical and Pharmacological Sciences

Expected Graduation Date - May 2017

2007 - $2011 \quad$ University of California, Los Angeles

Bachelor of Science - Physiological science

\section{RESEARCH EXPERIENCE}

2013 - Present $\quad$ Graduate Research - West Virginia University, Pharmacological and Pharmaceutical Sciences Graduate Program

Graduate Student

- Project: Evaluation of the Antidepressant Potential of Dextromethorphan

- Methods: behavioral tests (open field test, forced swim, tail suspension), cell culture (PC12), phase contrast microscopy, western blot, ELISA, protein assay, brain tissue collection, clinical trials, data analysis

2009 - $2011 \quad$ Undergraduate Research - UCLA, Department of Neurobiology

Undergraduate Research Student

- Project: The Effects of Common Pesticides on Ubiqituin-Activating E1 Ligase

- Methods: cell culture (3T3 cells), BCA protein assay, western blot, data analysis (Image $\mathrm{J}$ and Image Quant)

Summer 2010 BDBS Program - Tufts University, Department Pharmacology \& Experimental Therapeutics

Summer Research Student

- Project: The Effects of BMP Signaling on Catecholamine Release and Body Weight in Mice

- Methods: organ collection (adrenal glands), cell culture (chromaffin cells), carbon fiber amperometry

Summer 2009 SMART Program - University of Colorado Boulder, Department of Psychology \& Neuroscience

Summer Research Student

- Project: The Effect of Restraint Stress on the Activation of the CREB Coactivator TORC in the Hypothalamic Paraventricular Nucleus of the Adult Rat

- Methods: brain tissue collection and slicing, immunohistochemistry, radioimmunoassay, ELISA

2008 - $2009 \quad$ Undergraduate Research - UCLA, Division of Head \& Neck Surgery

Undergraduate Research Student

- Project: Quantification of Total Hair Cells in the Striolar region of Rat Utricle

- Methods: hair cell count (Neurolucida)

\section{OTHER WORK EXPERIENCE}


2013 - Present $\quad$ Peer Assisted Learning Tutoring Program - WVU School of Medicine

Tutor

2008 - $2011 \quad$ Academic Advancement Program - UCLA

Chemistry 14BL Peer Tutor

ORGANIZATIONS/LEADERSHIP ROLES

\begin{tabular}{|c|c|}
\hline 2014 - Present & $\begin{array}{l}\text { American Association of Pharmaceutical Scientists - Vice Chair } \\
\text { West Virginia University Student Chapter }\end{array}$ \\
\hline 2013 - Present & $\begin{array}{l}\text { Graduate Student Organization -- Member } \\
\text { West Virginia University }\end{array}$ \\
\hline 2013 - Present & $\begin{array}{l}\text { American Association of Pharmaceutical Scientists - Member } \\
\text { West Virginia University Student Chapter }\end{array}$ \\
\hline $2012-2013$ & $\begin{array}{l}\text { American Medical Women's Association -- Secretary } \\
\text { West Virginia University School of Medicine }\end{array}$ \\
\hline $2008-2011$ & $\begin{array}{l}\text { Bruin Run/Walk - Events Director } \\
\text { UCLA }\end{array}$ \\
\hline
\end{tabular}

VOLUNTEER/COMMUNITY SERVICE ACTIVITIES

$\begin{array}{ll}2013-\text { Present } & \begin{array}{l}\text { Student and Faculty Experience (SAFE) Clinic } \\ \text { WVU School of Medicine, Morgantown, WV }\end{array} \\ & \text { 5K Run for Cover Event Coordinator } \\ & \text { WVU School of Medicine American Medical Women's Association, Morgantown, WV } \\ & \begin{array}{l}\text { Habitat for Humanity Spring Break Volunteer } \\ 2011\end{array} \\ & \begin{array}{l}\text { WVU School of Medicine, Morgantown, WV } \\ \text { Child Life/Child Development Program Volunteer }\end{array} \\ 2008-2011 & \begin{array}{l}\text { CLCD Department, UCLA Ronald Reagan Hospital, Los Angeles, CA } \\ 2008-2011\end{array} \\ & \begin{array}{l}\text { Asian Pacific Health Corps at UCLA } \\ \text { UCLA, Los Angeles, CA }\end{array}\end{array}$

AWARDS/HONORS

2015 Behavioral and Biomedical Sciences (BBS) 2015 Training Scholarship

2015 Van Liere \& Research Day 2015 Poster Presentation, Basic Science, $1^{\text {st }}$ Place

$2015 \quad$ ASPET Graduate Student Travel Award

2014 WVU School of Pharmacy Research Forum Elevator Pitch Competition, $1^{\text {st }}$ Place

$2014 \quad$ Van Liere \& Research Day 2014 Poster Presentation, Basic Science, $1^{\text {st }}$ Place

$2014 \quad$ Behavioral and Biomedical Sciences (BBS) 2014 Training Scholarship

$2013 \quad$ Graduate Student Travel Awards for presentation at the SfN meeting

$2013 \quad$ Van Liere \& Research Day 2013 Poster Presentation, Basic Science, $1^{\text {st }}$ Place

$2011 \quad$ Graduated from UCLA summa cum laude

2007 Gates Millennium Scholarship

\section{PUBLICATIONS}

\section{Research Papers}

1. Xu Y, Pan J, Chen L, Zhang C, Sun J, Li J, Nguyen L, Nair N, Zhang H, O'Donnell JM. Phosphodiesterase-2 inhibitor reverses corticosterone-induced neurotoxicity and related behavioural changes via cGMP/PKG dependent pathway. Int J Neuropsychopharmacol, 2013 May: 16(4):835-47. (PMID: 22850435)

2. Matsumoto RR, Nguyen L, Kaushal N, Robson MJ. Sigma $(\sigma)$ receptors as potential therapeutic targets to mitigate psychostimulant effects. Adv Pharmacol, 2014: 69:323-386. (PMID: 24484982) 
3. Nguyen L, Robson MJ, Healy JR, Scandinaro AL, Matsumoto RR. Involvement of sigma-1 receptors in the antidepressant-like effects of dextromethorphan. PLoS ONE, 2014 Feb: 9: e89985. (PMID: 24587167)

4. Matsumoto RR, Seminerio MJ, Turner RC, Robson MJ, Nguyen L, Miller DB, O’Callaghan JP. Methamphetamine-induced toxicity: An updated review on issues related to hyperthermia. Pharmacol Ther, 2014 Oct: 144(1):28-40. (PMID: 24836729)

5. Lucke-Wold BP, DiPasquale K, Logsdon AF, Nguyen L, Lucke-Wold NA, Turner RC, Huber JD, Rosen CL. Metabolic syndrome and its profound effect on prevalence of ischemic stroke population. American Medical Student Research Journal, 2014.

6. Nguyen L, Kaushal N, Robson MJ, Matsumoto RR. Sigma receptors as therapeutic targets for neuroprotection. European Journal of Pharmacology, 2014 Nov: 15:42-147. (PMID: 25261035)

7. Nguyen L, Lucke-Wold BP, Mookerjee SA, Cavendish JZ, Robson MJ, Scandinaro AL, Matsumoto RR. Role of sigma-1 receptors in neurodegenerative diseases. Journal of Pharmacological Sciences, 2015 Jan: 127:17-29. (PMID: 25704014)

8. Nguyen L, Matsumoto RR. Involvement of AMPA receptors in the antidepressant-like effects of dextromethorphan. Behavioural Brain Research, 2015. In press.

9. Lucke-Wold BP, Turner RC, Logsdon AF, Nguyen L, Bailes JE, Lee JM, Robson MJ, Omalu BI, Huber JD, Rosen CL. Endoplasmic reticulum stress implicated in chronic traumatic encephalopathy. Journal of Neurosurgery, 2015. Accepted.

10. Smith K, Lucke-Wold, BP, Nguyen L, Turner RC, Logsdon AF, Jackson G, Huber J, Rosen C, Miller D. Sleep Disruption following Traumatic Brain Injury: Effects on Neuropsychiatric and Physical Symptoms. Neuroscience Biobehavioral Reviews, 2015. Accepted.

11. Nguyen L, Weaver CB, Cramer KJ, Pollard SE, Marshalek PJ, Matsumoto RR. Off-label use of transmucosal ketamine as a rapid acting antidepressant: a retrospective chart review. Neuropsychiatric Disease and Treatment, 2015. In revision.

12. Lucke-Wold BP, Nguyen L, Turner RC, Logsdon AF, Chen Y, Smith KE, Huber JD, Matsumoto RR, Rosen CL, Richter E. Traumatic Brain Injury and Epilepsy: Underlying Mechanisms Leading to Seizure. Epilepsia, 2015. In revision.

\section{Presentations}

1. Nguyen L, Weiser MJ, Spencer RL. The effects of restraint stress on the activation of the CREB-regulating coactivator TORC in the hypothalamic paraventricular nucleus of the adult rat. Poster presentation. Summer Multicultural Access to Research Training (SMART) Program Poster Day, Summer 2009, University of Colorado at Boulder, Boulder, CO.

2. Nguyen L, Cappellucci LA, Umberg EN, Geiger BM, Pothos EN. The effects of BMP receptor 1A on catecholamine exocytosis: Implications for obesity therapeutics. Poster presentation. Building Diversity in Biomedical Sciences (BDBS) Program Poster Day, Summer 2010, Tufts University, Boston, MA.

3. Nguyen L, Li E, Rinetti GV, Schweizer FE. The effect of pesticides on ubiquitin-activating enzyme of the ubiquitin proteasome system. Poster presentation. UCLA Science Poster Day, 2011, Los Angeles, CA.

4. Nguyen L, Robson MJ, Matsumoto RR. Dextromethorphan produces antidepressant-like effects in mice. Poster presentation. WVU Van Liere \& Research Day, 2013, Morgantown, WV.

5. Nguyen L, Robson MJ, Matsumoto RR. Dextromethorphan produces antidepressant-like effects in mice. Poster presentation. 5th Annual AAPS Pittsburgh Chapters Research Symposium, Oct 2013, Pittsburgh, PA.

6. Nguyen L, Robson MJ, Matsumoto RR. Dextromethorphan produces antidepressant-like effects in mice. Podia presentation. Society for Neuroscience, 2013, San Diego, CA.

7. Nguyen L, Matsumoto RR. Preclinical evaluation of the fast acting antidepressant potential of dextromethorphan: Involvement of AMPA and sigma-1 receptors. Poster presentation. WVU Van Liere Convocation \& Research Day, Feb 2014, Morgantown, WV.

8. Nguyen L, Matsumoto RR. Preclinical evaluation of the fast acting antidepressant potential of dextromethorphan: Involvement of AMPA and sigma-1 receptors. Poster presentation. WVU School of Pharmacy Research Forum, Jun 2014, Morgantown, WV.

9. Nguyen L, Matsumoto RR. Preclinical evaluation of the fast acting antidepressant potential of dextromethorphan: Involvement of AMPA and sigma-1 receptors. Podia \& poster presentations. WVU Center for Neuroscience Retreat, Jul 2014, Wheeling, WV. 
10. Nguyen L, Matsumoto RR. Preclinical evaluation of the fast acting antidepressant potential of dextromethorphan: Involvement of AMPA and sigma-1 receptors. Podia presentation. Society for Neuroscience, 2014. Washington, DC.

11. Nguyen L, Scandinaro AL, Logdson AF, Lucke-Wold BP, Matsumoto RR. Behavioral \& biochemical analyses of the fast acting antidepressant-like potential of dextromethorphan. Podia \& poster presentation. WVU Van Liere Convocation \& Research Day, Feb 2015, Morgantown, WV.

12. Nguyen L, Logsdon AF, Lucke-Wold BP, Matsumoto RR. Behavioral \& biochemical analyses of the fast acting antidepressant-like potential of dextromethorphan. Poster presentation. ASPET and Experimental Biology, Apr 2015, Boston, MA.

\section{LECTURES}

1. Nguyen L, Healy JR, Weaver C. Novel antidepressant therapies and opioid analgesics. Introduction to Research course for $1^{\text {st }}$ year WVU pharmacy students, 25 Sep 2014, Morgantown, WV.

2. Siderovski DP, Nguyen L. Antidepressants: Pharmacotherapy for mood disorders. Lecture to $1^{\text {st }}$ year WVU psychiatric residents, 9 Jul 2014, Morgantown, WV.

3. Nguyen, L. Ketamine and the next wave of antidepressants. Lecture to $1^{\text {st }}$ year WVU psychiatric residents, 30 Jul 30 2014, Morgantown, WV. 